HNF-SP-1112

Revision 4

\title{
Environmental Compliance Program FY 1999 Multi-Year Work Plan, WBS 1.8.2.3
}

\author{
K. A. Giese
}

Fluor Daniel Hanford, Inc.

Date published

August 1998

Prepared for the U.S. Department of Energy

FLUOR DANAER RANFOAD,

Richland, Washington 
LEGAL DISCLAIMER

This report was prepsed as an account of work sponsored by an agency of the United States Government. Neither the United States Government nor any agency thereof, nor any of their employees, nor any of their contractors, subcontractors or their emplovees, makes any wartanty. express or implied, or assumes any legal liabiliry or responsibility for the accuracy, completeness, or any third party's use or-the results of such use of any information, apparatus, product, or process disclosed, or represents that its use would not infringe privately owned rights. Reference herein to any specific commercial product, process, or service by trade name, trademark, manufacturer, or otherwise, does not necesserily constitute or imply its endorsement, recommendation, or favoring by the United States Government or any agency thereof or its contrectors or subcontractors. The views and opinions of authors expressed herein do not necessarily state or reflect those of the United States Government or any agency thereof.

This report has been reproduced from the best available copy.

Available in paper copy and microfiche.

Available to the U.S. Department of Energy and its contractors from

U.S. Department of Energy

Office of Scientific and Technical information (OSTI)

P.O. Box 62

Oak Ridge, TN 37831

(615) $576-8401$

Available to the public from the U.S. Department of Cammerce

National Tochnical information Service (NTIS)

5285 Port Royal Road

Springfield, VA 22161

(703) $487-4650$

Printed in the United States of America

DISCLM-1.CHP (8-95) 


\section{RELEASE AUTHORIZATION}

Document Number: HNF-SP-1112, Rev. 4

Document Title:

Environmental Compliance Program FY 1999 Multi-Year Work Plan, WBS 1.8.2.3

This document, reviewed in accordance with DOE Order 1430.1D, "Scientific and Technical Information Management," and DOE G 1430.1D-1, "Guide to the Management of Scientific and Technical Information," does not contain classified or sensitive unclassified information and is:

\section{APPROVED FOR PUBLIC RELEASE}

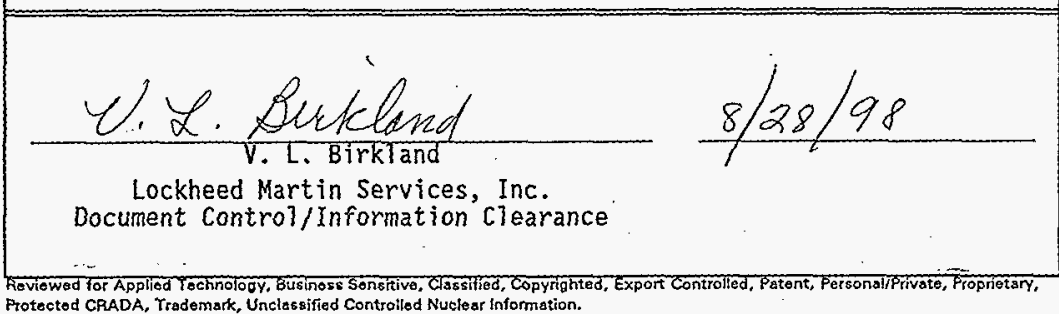

Ptotectod CRADA, Trademark, Unciassified Controlled Nutear Information.

LEGAL DISCLAMEER. This report was prepared as an account of work sponsored by an agency of the United States Govamment. Noither the United States Govemment nor any sgency thereof, not any of their emplayees, nor any of their cantractors, subcontractars of thoit employoes. makes any warranty, oxpross or implied, or assurnes any legal lisbility or responsibjlity for the aceuracy, complatoness, or any third party's use or the resules of such use of any information, apparatus, product, or procesa disciosed, or represents that its uso would not inftinge privatoly ownod nights. Roteronce hecoln to any spacific commercial product, process, of sarvice by trade name, tredemork, manufecturer, or otherwise, doo* not necaszarily constitute or imply its andorsement. recommendation, or fevoring by the United States Govemment or any eqency thereof or fte cantroctore or subcontractors. The views and opiniens of suthors expressed harein do not necessarily state or raflect those of the United States Govemmont or any agency thereof. This report hes bes reproduced from the best svollable copy. Printed in the United States of Americo. Avsilable to the U.S, Depertment of Enerey and te contrsctors from the U.S. Department of Energy Office af Scientific and Technkal information, P.O. Box 62. Cak Ridge, TN 37831; Tolephone: 423/576-8401.

Available to the publle from the U.S. Department of Commeres National Tochnical information Service. 5285 Port Roysl Rosd, Springfield, VA 22161; Tolephone: 703/487-4650. 
Environmental Compliance Program

HNF-SP-1112

FY 1999 Multi-Year Work Plan

WBS 1.8.2.3

U. S. Department of Energy

Richland Operations Office

ASSISTANT MANAGER-CONTRACTING OFFICERS REPRESENTATIVE
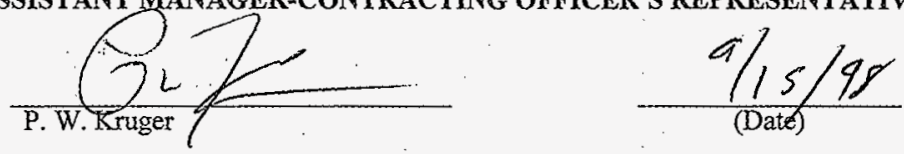

RE PROJECT CONTRACTING OFFICERS REPRESENTATIVE

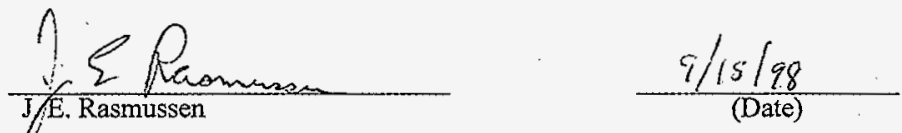

CONTRACTOR VICE PRESIDENT, OFFICE OF ENVIRONMENT, SAFETY AND HEALTH
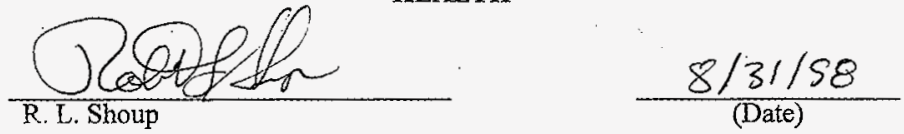

CONTRACTOR CONTRACTING OFFICERS REPRESENTATIVE
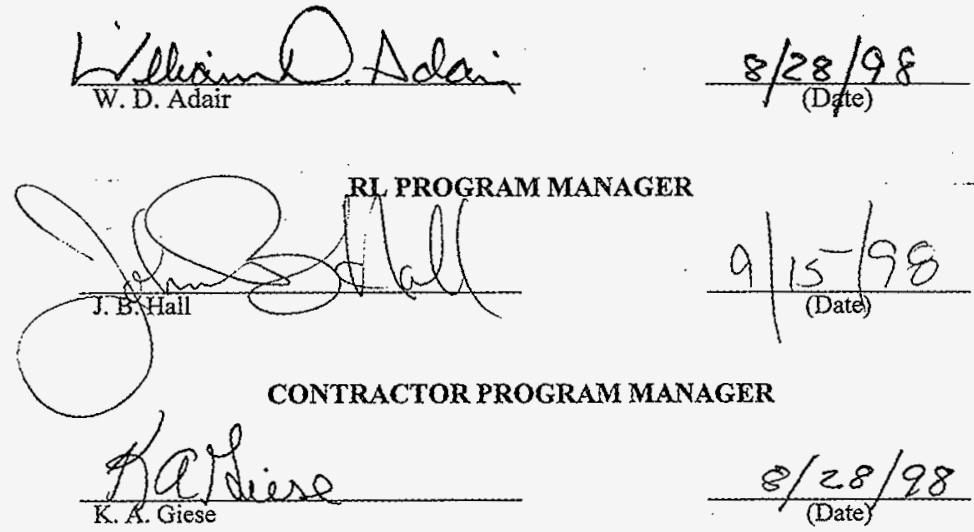


\section{TABLE OF CONTENTS}

1.0 Technical Baseline

1.1 Mission Statement

1.2 Boundary Diagram with Major Facilities ....................................................

1.3 Facility Responsibility Assignment Matrix ................................................

1.4 Project Planning Assumptions ...................................................................

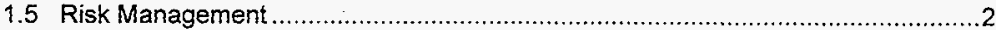

1.6. Technical lssues Manangement List ......................................................... 2

2.0 Project Hanford Breakdown Structure (PHBS) Hierarchy ................................2

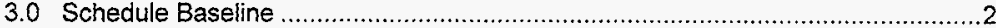

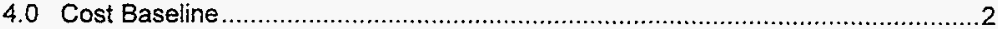

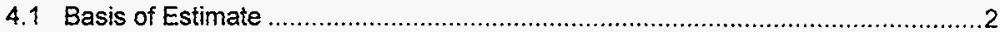

4.2 Summary of Life Cycle Cost Baseline (BCWS) by Project.....................................

4.3 Summary of Life Cycle Budget Authority (B/A) by Project .................................... 7

4.4 BCWS For Execution Year By Month By Fund Type ........................................9

4.5 BCWS For Execution Year By Month By Resource Type ...........................11 


\section{Project Summary Section}

\subsection{Technical Baseline}

The technical baseline describes the work to be accomplished and summarizes the technical standards which govern that work for the newly formed Environmental Compliance Program (ECP).

\subsection{Mission Statement}

ECP was formed by the combination of the Hanford Environmental Management Program (HEMP) and the Effluent and Environmental Monitoring Program (EEM).

The purpose of the ECP is to create and implement a structured approach toward achieving environmental compliance, and to provide environmental monitoring to determine the effects of site activities on the environment immediately adjacent to site facilities.

\subsection{Boundary Diagram with Major Facilities}

The Boundary Diagram with Major Facitities is not required for the Environmental Compliance Program.

\subsection{Facility Responsibility Assignment Matrix (RAM)}

The Facility Responsibility Assignment Matrix is not required for the Hanford Environmental Management Program.

\subsection{Planning Assumptions}

- Core Environmental Compliance Program work scope is driven by requirements to be in compliance with local, state, and federal regulations; the Tri-Party Agreement; and the NESHAPs Federal Facility Compliance Agreement.

- The Environmental Compliance Program supports the Hanford facilities in multifacility reporting by consolidating data to meet the intent of the regulatory drivers.

- The contractor, to the best of it's knowledge, will provide submittals of high quality that are the compilation of information received from it's major subcontractors. The contractor shall not be liable for any errors or omissions in information received, or the timeliness of such submittals from other prime contractors, unless the contractor, as site integrator, fails to notify DOE-RL if another site prime contractor will not meet deliverables that allow the contractor to meet submittal dates with DOE-RL.

- The Environmental Compliance Program will actively maintain, identify, and disclose in conjunction with other contractors a complete up-to-date inventory of potential crosscutting noncompliances so that their resolution can be funded. If funds are not available within the signed Work Authorization, the contractor will propose an action plan to properly disposition the noncompliance conditions consistent with the provisions of the Tri-Party Agreement. 


\subsection{Risk Management}

The Environmental Compliance Program is developing and implementing a PHMC-wide chemical management system with the goal being to: 1) Manage and control chemicals from procurement through use and final disposition; 2) Develop and maintain procedures for identifying and evaluating hazards and environmental impacts present in facilities, and the hazard classification of the facilities.

The U.S. Department of Energy (DOE) may promulgate the final rule, 10 CFR 834 , "Radiation Protection of the Public and the Environment" in FY 1999. This rule establishes controls for the release of radioactive material and limits for the amount of radiation exposure to the public and the environment. It will be applicable to activities of DOE contractors at the Hanford site. This rule is expected to replace the bulk of DOE Orders 5400.5, "Radiation Protection of the Public and the Environment" and 5400.1, "General Environmental Protection Program". In doing so, these Orders will be backed by the Price-Anderson enforcement procedures and carry penalties for non-compliance.

\subsection{Technical Issues Management List}

There were no site level technical issues found for the Mission Support Organization, which includes the Environmental Compliance Program.

\subsection{PHBS}

The PHBS Hierarchy to the PBS level is provided. The Environmental Compliance Program is one of four program elements within the single PBS labeled Mission Support.

\begin{tabular}{lll} 
RL PBS & RL. WBS & TITLE \\
\hline & 1.08 & SUpport \\
\hline RL-OTO1 & 1.08 .02 & Mission Support - Other Multi-Year Programs \\
\hline & 1.08 .02 .01 & Site Planning and Integration \\
1.08 .02 .02 & Systems Engineering \\
& 1.08 .02 .03 & Environmental Compliance Program \\
& 1.08 .02 .04 & Public Safety and Resource Protection
\end{tabular}

\subsection{Schedule Baseline}

The execution year program-level schedule, and the lifecycle schedule are located in the Environmental Compliance Program Detailed section.

\subsection{Cost Baseline}

\subsection{Basis of Estimate}

The Environmental Compliance Program cost estimates were prepared and documented according to the requirements set forth in HNF-PR-000585, which is the Activity Based Cost ( $A B C$ ) estimating method. The $A B C$ method was used to estimate 
$100 \%$ of the program dollars. The ABC definitive method was used. Due to the nature of work in the Environmental Compliance Program, some of the work for most of the accounts are deemed level of effort. Planning rates for the execution year were consistenf with the site wide planning rates. Escalation rates for the outyears were consistent with the PBS submittal for the Environmental Compliance Program.

Cost estimates for FY 1999 were prepared at the activity level in all cases, and rolled up to the cost account level. Cost estimates for FY 1999 were consistent with the President's budget.

Final cost estimate packages assembled into basis of estimate (BOE) books for the Environmental Compliance Program, are located at the 2440 Stevens Center, Richland Washington, room 2215. Contact is Ken Giese (PH 509-376-8301, Fax 509-372-2828).

Final BOE books contain the following: (Note: All are required per HNF-PR-000585, Cost Estimating.)

- Technical scope

- Purpose of the project

- Assumptions and exclusions

- Estimate work breakdown structure

- Technique and historical basis

- Cost estimating relationship (CER) development

- Labor rates, unit rates, and unit cost sources

- Escalation amounts and sources

- Contingency calculation development (if applicable)

- Legal drivers

- Labor costs

- Technical logic diagram (if applicable)

- Integrated, logic driven, resource loaded schedules

- Responsibility assignment matrix

- Any other costs that will be reflected in the budget for the project/program

\subsection{Summary of Life Cycle Cost Baseline (BCWS) by Project}

The data in Exhibit 1 provides a life cycle summany of BCWS by PBS at the mission summary level for each fiscal year from 1998 through project completion. Estimated carryover is not included at this time. Please see the figure on the following page. 


\section{SUPPORT}

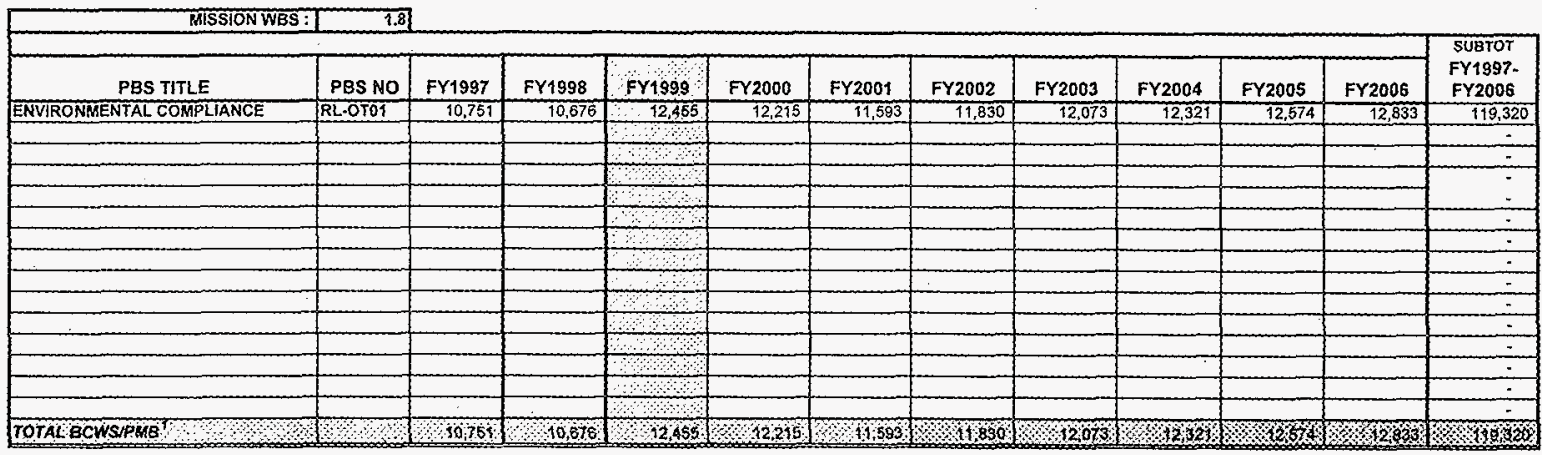

${ }^{1}$ Budgeted Cost of Work Scheduled (BCWS) Equals Performance Measurement Baseline (PMB); Expense Carryover is NOT Included. 
EXHEIT \&

\section{SUMMARY OF LIFE CYCLE COST BASELINE (BCWS) BY YEAR}

BY PROJECT BASELINE SUMMARY (PBS)

FY 1999

(\$000s)

SUPPORT

\begin{tabular}{|c|c|c|c|c|c|c|c|c|c|c|c|c|}
\hline \multicolumn{13}{|c|}{ MISSIONWBS:I } \\
\hline PRS TIT F & PBSNO & & & & & FY2011- & FY2016- & FY20210 & FY2026- & FY2031. & FY2036 & $\begin{array}{l}\text { FY2041- } \\
F Y 2045\end{array}$ \\
\hline $\begin{array}{l}\text { PBS TITLE } \\
\text { ENVIRONMENTAL COMPLIANCE }\end{array}$ & $\frac{\text { PBS NO }}{\text { RL-OTO1 }}$ & $\frac{F Y 2007}{13,097}$ & $\frac{\mathrm{FY} 2008}{13,367}$ & $\begin{aligned} F Y 2009 \\
13,644\end{aligned}$ & $\frac{F Y 2010}{13,926}$ & $\frac{F Y 2010}{74,088}$ & $\frac{172020}{82,138}$ & $\frac{P 1200}{91.114}$ & $\frac{1+12030}{82.144}$ & $\frac{12030}{44.234}$ & 48,853 & $\begin{array}{r}512040 \\
-54,003 \\
\end{array}$ \\
\hline & & & & & & & & & & & & \\
\hline & & & & & & & & & & & & \\
\hline & & & & & & & & & & & & \\
\hline & & & & & & & & & & & & \\
\hline & & & & & & & & & & & & \\
\hline & & & & & & & & & & & & \\
\hline & & & & & & & & & & & & \\
\hline & & & & & & & & & & & & \\
\hline TOTAL BCWSPMA & $\because 1$ & 13,007 & $13,36 \%$ & 13,69 & 13,826 & (1, 4,068 & 82,33 & 080119 & $0 \times 2,14$ & $4,2,4$ & 1865 & $3 \times 5060$ \\
\hline
\end{tabular}

'Budgeted 18udgeted Cost of Work Scheduled (8CWS) Equals Performance Measurement Baseline (PMB); Expense Carryaver Is NOT Included. 
SUMMARY OF LIFE CYCLE COST BASELINE (BCWS) BY YEAR BY PROJECT BASELINE SUMMARY (PBS)

FY 1999

(\$000s)

\begin{tabular}{|c|c|c|c|}
\hline \multicolumn{3}{|c|}{ MISSTON WBS: 1 _.81 } & \multirow{3}{*}{$\begin{array}{l}\text { TOTAL } \\
\text { FY1997- } \\
\text { FY2050 }\end{array}$} \\
\hline & & & \\
\hline PBS TITLE & PBS NO & FY2050 & \\
\hline ENVIRONMERTAL COMPLIANCE & RL.OTOT & 59,745 & 709,672 \\
\hline & & & \\
\hline & & & \\
\hline & & 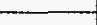 & : \\
\hline & & & $\div$ \\
\hline & & & $=$ \\
\hline & & & - \\
\hline & & & $\therefore$ \\
\hline & & & $\because$ \\
\hline & & & \\
\hline & & & $\therefore$ \\
\hline & & & $\div$ \\
\hline TOTAL BCWSIPMB & & $\$ 9,7,45$ : & 3709,672 \\
\hline
\end{tabular}


HNF-SP-1112, Rev. 4

\subsection{Summary of Life Cycle Budget Authority (B/A) by Project}

The data in Exhibit 4 provides a comparison of BIA by PBS at the project summary level for fiscal year 1998 and 1999 . Estimated carryover is currently not included in the cost baseline. Please see the figure on the following page. 


\section{SUPPORT}

SUMMARY OF BUDGET AUTHORITY (B/A) BY YEAR

BY PROJECT BASELINE SUMMARY (PBS)

FY 1999

(\$000s)

\begin{tabular}{|c|c|c|c|c|}
\hline & & & & \\
\hline & $\begin{array}{c}\text { TARGET } \\
\text { BIA }\end{array}$ & $\begin{array}{l}\text { CARRYOVER } \\
\text { FROM }\end{array}$ & $\begin{array}{l}\text { PRESIDENT'S } \\
\text { BUDGET }\end{array}$ & $\begin{array}{l}\text { PRESIDENT'S } \\
\text { BUDGET PLUS }\end{array}$ \\
\hline PBS TITLE & FY4998 & FY1998 & FY1999 & $\begin{array}{l}\text { CARRYOVER' } \\
\text { FY1999 }\end{array}$ \\
\hline ENVIRONWENTAL COMPLIANCE & 10,085 & - & 12,455 & 12,455 \\
\hline & & & & \\
\hline & & & & \\
\hline & & & $\because$ & \\
\hline & & & & \\
\hline & & & & \\
\hline & & & & \\
\hline & & & & \\
\hline & & & & \\
\hline & & & & $\therefore$ \\
\hline & & & & \\
\hline & & & & \\
\hline & & & & \\
\hline & & & & \\
\hline & & & & \\
\hline TOTAL B/A & 10,085 & - & 12,455 & 12,455 \\
\hline
\end{tabular}

'Estimated Carryover Is NOT Included In Cost Baseline; Change Request For Actual Carryover Submitted After September 30, 1998. 


\subsection{BCWS for execution year by month by fund type}

The data in Exhibit 2 identifies project execution year BCWS by fund type by month. Due to the new financial system, this cost exhibit cannot be completed at this time. It is estimated that this information will be available around November, 1998. The BCWS will not include Management Reserve or Line Item contingency held by RL. Estimated carryover will also not be included. Please see the figure on the following page. 
SUMMARY OFFY 1999 COST BASELINE (BCWS) BY MONTH

BY FUND TYPE BY PROJECT

EXECUTION YEAR

(\$0005)

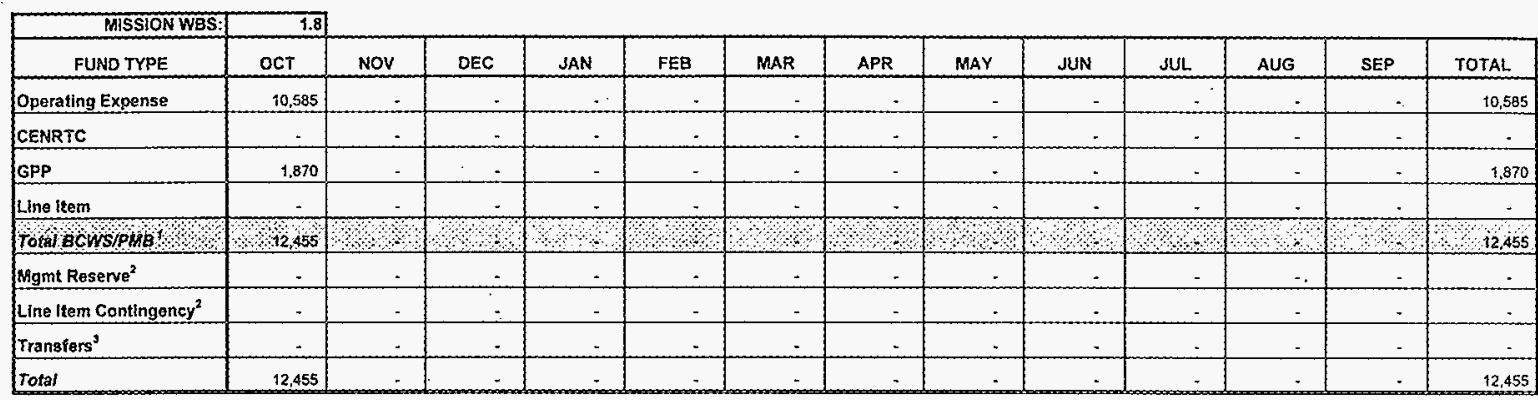

'Budgeted Cost of Work Schedulgd (BCWS) Equals Pertormance Measuremen! Baseling (PMB); Expensa Carryover Is Mot Included

${ }^{2}$ Management Reservo end Lino liem Contingency Hold $8 y$ RL

'FundsWorkscope Tranisferted to Other Sites, Transforred to Hanford from Other Sïtes. and/or Fundsisoskscopo Controlled by RL. 


\subsection{BCWS for execution year by month by resource type}

The data in Exhibit 3 identifies project execution year BCWS by cost by month by resource type. Due to the new financial system, this cost exhibit cannot be completed at this time. It is estimated that this information will be available around November, 1998. The BCWS will not include Management Reserve or Line Item contingency held by RL. Estimated carryover will also not be included. Please see the figure on the following page. 
HNF-SP-1112, Rev. 4

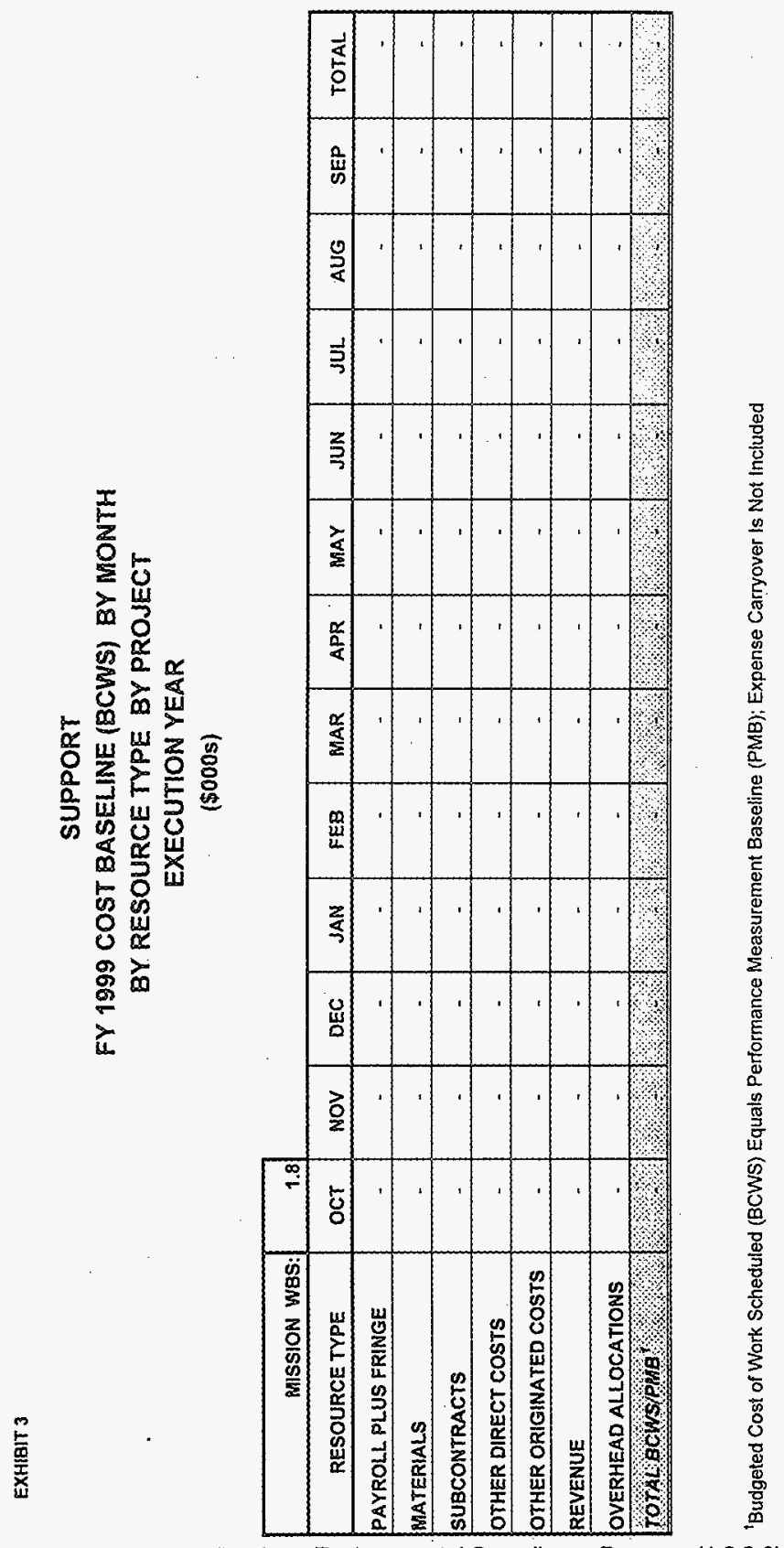

Summary Section - Environmental Compliance Program (1.8.2.3) Page 12 of 12 


\section{TABLE OF CONTENTS}

Environmental Compliance Program Detailed Section

1.1 Project Mission......................................................................................... 1

1.2 Project End Point Targets .......................................................................

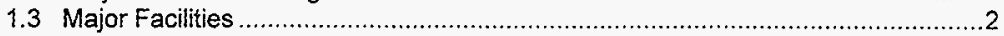

1.4 Drivers ..............................................................................................

2.0 Work Breakdown Structure (WBS) ......................................................

2.1 WBS Hierarchy for the Environmental Compliance Program ............................ 5

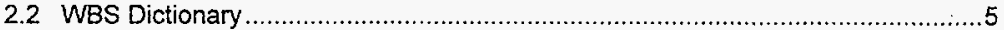

2.3 Responsibility Assignment Matrix (RAM) .............................................. 18

3.0 Schedule Baseline ............................................................................. 19

3.1 Project Master Baseline Schedule (P.MBS) .............................................. 19

3.2 Execution Year PMBS ..................................................................... 21

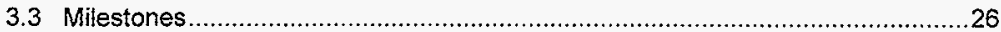

3.4 Milestone Description Sheets - Execution Year ...........................................29

3.5 Milestone Description Sheets - Outyears .............................................. 85

4.0 Cost Baseline ..................................................................................... 136

4.1 Life Cycle Cost Baseline (BCWS) by PBS ........................................... 136

4.2 Life Cycle Budget Authorify (B/A) by PBS .............................................. 140

4.3 Execution Year Cost Baseline by Month by PBS by Fund Type ..................... 142 
HNF-SP-1112, Rev. 4

\section{Environmental Compliance Program Detailed Section}

\subsection{Technical Baseline}

The technical baseline describes the work to be accomplished and summarizes the technical standards which govern that work for the Environmental Compliance Program.

\subsection{Project Mission}

ECP was formed by the combination of the Hanford Environmental Management Program (HEMP) and the Effiuent and Environmental Monitoring Program (EEM).

The old Hanford Environmental Management Program was established to create and implement a structured approach toward achieving environmental compliance. The purpose was to provide guidance and support across Hanford Site contractors to ensure facilities/programs achieve compliance with environmental requirements and agreements, and to provide $\mathrm{RL}$ with a mechanism to coordinate specific environmental activities between ifs contractors. For site wide reports and permits, the contractor coordinates and integrates the activities of Hanford Site contractors as directed by RL. and subject to cooperation by the other prime contractors. For regulator access, the coniractor provides a focal point for regulator data needs and issues.

The old Effluent and Environmental Monitoring (EEM) Program provides effluent and environmental monitoring which also includes near-field monitoring, to determine the effects of site activities on the environment immediately adjacent to site facilities. The EEM program manages NESHAP compliance activities on the Site. The program provides safety measures to protect workers, the public, the environment; and verifies that these measures are effective. The program also provides a basis for the initiation of corrective measures, if required. The program will accomplish this work scope through teamwork between DOE and its contractors, the regulators, technical experts, and the public.

Environmentai compliance support is required for the contractor and RL to maintain compliance with environmental requirements and agreements throughout Project Hanford cleanup. As compliance requirements change, these activities will be revised to ensure that compliance is achieved to the extent funding allows. Facility or other contractor specific compliance activities remain the responsibility of individual programs. The nature and extent of these activities will be modified as the cleanup mission progresses and decisions are made regarding cleanup strategy.

\subsection{Project End Point Targets}

The Environmental Compliance Program contains a variety of activities, some that have project end point targets and some that do not have project end point targets. For those activities that do not have end point targets, there is written direction to continue monitoring at the Hanford Site for 20 years after cessation of Hanford Site activities/operations; currently estimated to be until 2070. For those activities that do have project end point targets such as various permitting and reporting activities, it is anticipated that the end point target parallels the accomplishment of the Hanford Site Mission, which, for planning purposes, is assumed to be in the year 2050 . 


\subsection{Major Facilities}

The Major Facilities section is not required for the Environmental Compliance Program.

\subsection{Drivers}

Program Direction/Management Manage the Environmental Compliance Program in accordance with the provision of the contract. Develop and implement strategies that support Contractor and RL compliance with specific environmental requirements and agreements that crosscut Hanford Site programs.

Drivers:

DOE Order 4700.1

NEPA Services Provide RL with support to management and facilities on overall NEPAVSEPA activities to ensure consistency and compliance. Primary activities include updating the "NEPA Source Guide", maintenance of NEPA compliance files and databases, collecting and disseminating general NEPASEPA compliance information to Hanford Site management and facilities, evaluating and commenting on draft DOE NEPA guidance and programmatic EISs, coordinating cultural and biological reviews with NEPA review, integration of NEPA review with contractor site planning processes (EMS, ISMS, CERCLA), and updating of NEPA training and training materials for the Hanford Site.

Drivers:

40 CFR 1500-1508; 10 CFR 1021; 10 CFR 1022; 36 CFR 800; 36 CFR 63; 16 USC 461-467;16 USC 1531; 42 USC 4321; DOE Order 4320.1B (7)(1); DOE Order 451; Executive Order 11988; Executive Order 11990; Executive Order 12898, A Federal Actions to Address Environmental Justice in Minority Populations and Low-Income Populations;@RCW 43.21C; WAC 197-11-960

Compliance Integrațion and Process Improvement Provide coordination and facilitation of onsite inspections by regulatory agencies. Coordinate and integrate the resolution of crosscutting environmental noncompliance conditions. Serve as focal point for resolution of Contractor dangerous waste management issues.

Drivers:

WAC 173 303; RCW 70.105; DOE Order 5400.2A; Tri-Party Agreement

Air and Water Permit Services Prepare crosscuiting Air and Water permit application documentation. Participate in negotiations with federal, state, and local regulatory agencies in the establishment of permitting priorities, schedules, and resolution of permitting issues, compliance plans; and agreements involving Contractor Air and Water permitsing activistes. Coordinate and integrate Hanford Site Air Operating Permit activities. Perform Air and Water permitting coordination and documentation consolidation for the Hanford Site as directed by $\mathrm{RL}$ and subject to cooperation by the other contractors.

Drivers:

Clean Air Act (CAA); Clean Water Act (CWA); 40 CFR 52, 60, 61, 63, 70, 80, 82; 40 CFR 122; 40 CFR 264/265; 40 CFR 372;WAC 173-216; WAC 173-218; 


\subsection{Drivers (Cont.)}

WAC 173-221; WAC 173-303; WAC 173-400; WAC 173-401; WAC 173-425; WAC 173-460; WAC 173-480; WAC 246-247; WAC 246-290; Consent Order No. DE $91 \mathrm{NM}$-177; DOE Order 5400.1; DOE Order 5400.5; DOE Order 5484.1; RCW 70.94.431

Site Wide Environmental Reports Prepare documentation required for monitoring and reporting hazardous chemical information under the Emergency Planning and Community Right-To-Know Subtitle B regulations. Ensure compliance with Hanford Site reporting requirements for dangerous and/or hazardous waste and toxic substances as specified by environmental regulations and statufes. Prepare an annual status report on Hanford LDR mixed waste as required under the TPA. Perform Environmental Reporting coordination and documentation consolidation for the Hanford Site as directed by $\mathrm{RL}$ and subject to cooperation by the other contractors.

Drivers:

29 CFR 1910.1200; 40 CFR 260, 264.75, $265268,302,355,370,372,761.180$, 761 Subpart K; WAC 173-303-140, -145, -220, -390; RCRA 3016; CERCLA 102, 103; FFCA of 1992; Tri-Party Agreement M-26-01

Tri-Party Agreement integration Provide technical and administrative support to $\mathrm{RL}$ in coordinating and integrating the Tri-Party Agreement requirements. Perform Tri-Parfy Agreement coordination and documentation consolidation for the Hanford Site as directed by RL and subject to cooperation by the other contractors. Oversee the development and maintenance of guidelines and procedures necessary for implementation of the Tri-Pariy Agreement. Participate in resolution of technical issues related to Tri-Party Agreement activities.

Oversee the operation and maintenance of the administrative records and public information repositories required by the Tri-Party Agreement. Prepare the TriParty Agreement annual update, quarterly reports, and provide status and progress on the Tri-Party Agreement milestones. Assist in the coordination of Tri-Party Agreement public meetings. Provide Ecology and EPA secured access to the Hanford Local Area Network so as to render direct access to the Contractor and other contractors databases that are relevant to work performed under the Tri-Party Agreement.

Drivers:

Tri-Party Agreement

RCRA Services Prepare crosscutting RCRA permit application documentation. Participate in negotiations with federal, state, and local regulatory agencies in the establishment of permitting priorities and schedules, in the resolution of permitting issues, and in the development of compliance plans and agreements involving Contractor permitting activities. 


\subsection{Drivers (Cont)}

Coordinate and integrate Hanford Facility RCRA Permit activities. Perform RCRA permitting coordination and documentation consolidation for the Hanford Site as directed by RL and subject to cooperation by the other contractors. Support resolution of cross-cutting TSCA (PCB) and RCRA compliance issues. Per the Hanford Facility RCRA Permit, DOE-RL and contractors who manage treatment, storage, and/or disposal units are defined as Permittees.

Drivers:

WAC 173-303; RCW 70.105; Hanford Facility RCRA Permit; Tri-Panty Agreement

Clean Air Act (CAA) - Both radioactive and non-radioactive air emissions are discharged to the atmosphere from facilities at Hanford. Specific monitoring and reporting are required by this Act.

Federal Facility Compliance Agreement - National Emission Standards for Hazardous Air Pollutants (NESHAP) requirements have been activated for Hanford stacks. The FFCA requires upgrades to monitoring systems of all major stacks.

Washington State Administrative Code (WAC) -- The WAC defines restrictions on discharges of effluents and liquids.

\section{Other Regulatory Drivers}

40 CFR 265

Interim Status Standards for Owners and Operators of Hazardous Waste Treatment, Storage, and Disposal Facilities

40 CFR 61

National Emission Standards for Hazardous Air Pollutants

WAC-173-303

Dangerous Waste Regulations 
The technical baseline is directly related to the schedule and cost estimates through a WBS. The WBS section shall identify the work scope and a summary of the necessary activities to provide services and products to the customer. The Responsibility Assignment Matrix (RAM) identifies the organizations responsible for accomplishing the work scope.

\subsection{WBS Hierarchy for the Environmental Compliance Program}

This section provides the basic WBS hierarchy for the Environmental Compliance Program, which, as stated previously in the Summary Section, is one of the programs under Mission Support.

\begin{tabular}{lll} 
RL PBS & RL WBS & TITLE \\
\hline & 1.08 & Support \\
\hline RL-OT01 & 1.08 .02 & Mission Support - Other Multi-Year Programs \\
& 1.08 .02 .03 & Environmental Compliance Program \\
& 1.08 .02 .03 .01 & Program Management \\
& 1.08 .02 .03 .02 & NEPA \\
1.08 .02 .03 .03 & Compliance Integration and Process Improvement \\
1.08 .02 .03 .04 & Air and Water Services \\
1.08 .02 .03 .05 & Site wide Environmental Reports \\
1.08 .02 .03 .06 & TPA Integration \\
1.08 .02 .03 .07 & RCRA Services \\
1.08 .02 .03 .08 & Effluent and Environmental Monitoring \\
1.08 .02 .03 .09 & NESHAP Compliance \\
1.08 .02 .03 .10 & Environmental Surveillance (HPT) of Stabilized Sites. \\
1.08 .02 .03 .11 & W-420 Stack Monitoring System Upgrades \\
1.08 .02 .03 .12 & Other Project Activities in Support of Project W-420
\end{tabular}

\subsection{WBS Dictionary}

This program is divided into twelve cost accounts as previously diagrammed in Section 2.1 "WBS Hierarchy for the Environmental Compliance Program". Following is a summary level description of the work scope in each of these cost accounts. Please see the figures on the following pages. The detailed technical work scope backup information for each cost account can be found in the Basis of Estimate (BOE) books for the Environmental Compliance Program, located at 2440 Stevens Center, Richland Washington, room 2215. Contact is Ken Giese (PH 509-376-8301, Fax 509-372-2828). 


\begin{tabular}{|c|c|c|c|}
\hline $\begin{array}{l}\text { Dictionary Title: } \\
\text { Environmental } \\
\text { Compliance } \\
\text { Program }\end{array}$ & $\begin{array}{l}\text { 2. Date: } \\
08 / 04 / 98\end{array}$ & $\begin{array}{l}\text { 3. PBS Number: } \\
\text { RL-OTO1 }\end{array}$ & \\
\hline $\begin{array}{l}\text { 5. WBS No.: } \\
1.8 .2 .3 .1\end{array}$ & $\begin{array}{l}\text { 6. B\&R NO.: } \\
\text { EW0290700 }\end{array}$ & \multicolumn{2}{|c|}{ 7. Baseline CR No:: } \\
\hline \multicolumn{4}{|c|}{$\begin{array}{l}\text { 8. Organization Name: Environmental Compliance Program } \\
\text { 9. Scope of Work: }\end{array}$} \\
\hline \multicolumn{4}{|c|}{ Program Direction/Management Program (1.8.2.3.1), FDS WBS 1R1H01: } \\
\hline \multicolumn{4}{|c|}{$\begin{array}{l}\text { Program Management provides a focus of responsibility and authority for managing the } \\
\text { Environmental Compliance Program. Program priorities are established and } \\
\text { administrative support is provided for program reporting requirements/documentation, } \\
\text { funds management, and commitment schedules. Program goals include, but are not } \\
\text { limited to, managing the Environmental Compliance Program in accordance with the } \\
\text { provisions of the Contract, and continuing to improve the efficiency and effectiveness of } \\
\text { the Program. }\end{array}$} \\
\hline \multicolumn{4}{|c|}{$\begin{array}{l}\text { The program develops and implements strategies that support Contractor and DOE-RL } \\
\text { compliance with specific environmental requirements and agreements that crosscut } \\
\text { Hanford Site programs. The program provides DOE-RL with a mechanism to coordinate } \\
\text { activities between contractors including Hanford Site activities for site wide reports and } \\
\text { permits, TPA coordination, and provides a focal point for regulator data needs and } \\
\text { issues. }\end{array}$} \\
\hline \multicolumn{4}{|c|}{$\begin{array}{l}\text { Deliverables to DOE-RL include monthly progress reports via the Progress Tracking } \\
\text { System (PTS), out year budget planning documents such as the Project Baseline } \\
\text { Summary (PBS), and the annual Multi-Year Work Plan (MYWP). }\end{array}$} \\
\hline
\end{tabular}




\begin{tabular}{|c|c|c|c|}
\hline $\begin{array}{l}\text { 1. Dictionary Title: } \\
\text { Environmental } \\
\text { Compliance } \\
\text { Program }\end{array}$ & $\begin{array}{l}\text { 2. Date: } \\
08 / 04 / 98\end{array}$ & $\begin{array}{l}\text { 3. PBS Number: } \\
\text { RL-OT01 }\end{array}$ & $\begin{array}{l}\text { 4. Dict Rev: } \\
0\end{array}$ \\
\hline $\begin{array}{l}\text { 5. WBS No.: } \\
1.8 .2 .3 .2\end{array}$ & $\begin{array}{l}\text { 6. B\&R No.: } \\
\text { EWO290700 }\end{array}$ & \multicolumn{2}{|c|}{ 7. Baseline CR No.: } \\
\hline \multicolumn{4}{|c|}{ 8. Organization Name: Environmental Compliance Program } \\
\hline \multicolumn{4}{|c|}{ 9. Scope of Work: } \\
\hline \multicolumn{4}{|c|}{ NEPA Services (1.8.2.3.2), FDS WBS 1R1H02: } \\
\hline \multicolumn{4}{|c|}{$\begin{array}{l}\text { Provide DOE-RL with support to management and facilities on overall NEPAISEPA } \\
\text { activities to ensure consistency and compliance. Primary activities include updating the } \\
\text { "NEPA Source Guide", maintenance of NEPA compliance files and databases, collecting } \\
\text { and disseminating general NEPASEPA compliance information to Hanford Site } \\
\text { management and facilities, evaluating and commenting on draft DOE NEPA guidance } \\
\text { and programmatic EISs, coordinating cultural and biological reviews with NEPA review, } \\
\text { integration of NEPA review with contractor site planning processes, and provide NEPA } \\
\text { training and training materials for the Hanford Site. }\end{array}$} \\
\hline \multicolumn{4}{|c|}{$\begin{array}{l}\text { These items suppor NEPA compliance throughout the contractor organizations and } \\
\text { allow for information on existing approved NEPA documentation to be readily available } \\
\text { to contractors and DOE staff on the Hanford Site. }\end{array}$} \\
\hline \multicolumn{4}{|c|}{$\begin{array}{l}\text { Deliverables to RL include a biannual NEPA Activities Status Report, an updated NEPA } \\
\text { Source Guide and categorical exclusion requests, and updates of Hanford Site wide CXs } \\
\text { when requested. }\end{array}$} \\
\hline
\end{tabular}




\begin{tabular}{|c|c|c|c|}
\hline $\begin{array}{l}\text { 1. Dictionary Title: } \\
\text { Environmental } \\
\text { Compliance } \\
\text { Program }\end{array}$ & $\begin{array}{l}\text { 2. Date: } \\
08 / 04 / 98\end{array}$ & $\begin{array}{l}\text { 3. PBS Number: } \\
\text { RL-OTO1 }\end{array}$ & $\begin{array}{l}\text { 4. Dict Rev: } \\
0\end{array}$ \\
\hline $\begin{array}{l}\text { 5. WBS No.: } \\
1.8 .2 .3 .3\end{array}$ & $\begin{array}{l}\text { 6. B\&R NO:: } \\
\text { EW0290700 }\end{array}$ & \multicolumn{2}{|l|}{ 7. Baseline CR No: } \\
\hline \multicolumn{4}{|c|}{ 8. Organization Name: Environmental Compliance Program } \\
\hline \multicolumn{4}{|c|}{ Compliance integration and Process Improvement (1.8.2.3.3) FDH WBS 1R1H03: } \\
\hline \multicolumn{4}{|c|}{$\begin{array}{l}\text { Develop PHMC-wide and site wide environmental policy and compliance strategies. } \\
\text { Facilitate regulatory interactions and compliance, including coordination of } \\
\text { environmental inspections, release reporting, and permitting, review and integration of } \\
\text { environmental documentation, coordination of permit implementation, and coordination } \\
\text { of compliance issue resolution. Develop, implement, and improve environmental } \\
\text { management systems (e.g., Integrated Safety Management System, Chemical } \\
\text { Management System). Management of this account includes development and } \\
\text { maintenance of the MYWP, monthly reporting, and cost management. This account } \\
\text { includes administrative costs for all activities under } 1 \mathrm{R} 1 \mathrm{H} 03 \text { (e.g., training, office } \\
\text { supplies, multimedia services, computer hardware/software, and P-Card purchases.) }\end{array}$} \\
\hline \multicolumn{4}{|c|}{$\begin{array}{l}\text { The work scope develops and implements sirategies that support Contractor and RL } \\
\text { compliance with specific environmental requirements and agreements that crosscut } \\
\text { Hanford Site programs. The work scope provides RL with a mechanism fo coordinate } \\
\text { activities between contractors including Hanford Site activities for site wide reports and } \\
\text { permits, and provides a focal point for regulator data needs and issues. }\end{array}$} \\
\hline \multicolumn{4}{|c|}{$\begin{array}{l}\text { Deliverables to DOE-RL include: (1) Quarterly status report of NOVs; (2) monthly } \\
\text { inspection report based on PHMS inspections; (4) Complete Annual lnspections of the } \\
100,200,300,400 \text {, and } 1100 \text { Areas per RCRA permit condition II.0; (5) quarterly } \\
\text { Notification of Class } 1 \text { modifications package for the Hanford Facility (HF) RCRA Permit; } \\
\text { (6) Certified Class } 3 \text { modification package for the HF RCRA Permit; and (7) transmittal of } \\
\text { completion letter to DOE-RL for the Annual Asbestos Notification to the BCAA. }\end{array}$} \\
\hline
\end{tabular}




\begin{tabular}{|c|c|c|c|}
\hline $\begin{array}{l}\text { 1. Dictionary Title: } \\
\text { Environmental } \\
\text { Compliance } \\
\text { Program }\end{array}$ & $\begin{array}{l}\text { 2. Date: } \\
08 / 04 / 98\end{array}$ & $\begin{array}{l}\text { 3. PBS Number: } \\
\text { RL-OT01 }\end{array}$ & $\begin{array}{l}\text { 4. Dict Rev: } \\
0\end{array}$ \\
\hline $\begin{array}{l}\text { 5. WBS No.: } \\
1.8 .2 .3 .4\end{array}$ & $\begin{array}{l}\text { 6. B\&R No.: } \\
\text { EWO290700 }\end{array}$ & \multicolumn{2}{|c|}{ 7. Baseline CR No:: } \\
\hline \multicolumn{4}{|c|}{$\begin{array}{l}\text { 8. Organization Name: Environmental Compliance Program } \\
\text { 9. Scope of Work: }\end{array}$} \\
\hline \multicolumn{4}{|c|}{$\begin{array}{l}\text { 9. Scope of Work: } \\
\text { Air and Water Services (1.8.2.3.4), FDS WBS 1R1H04: }\end{array}$} \\
\hline \multicolumn{4}{|c|}{$\begin{array}{l}\text { Prepare crosscutting Air and Water permit application documentation. Participate in } \\
\text { negotiations with federal, state, and local regulatory agencies in the establishment of } \\
\text { permitting priorities, schedules, and resolution of permitting issues, compliance plans, } \\
\text { and agreements involving Contractor Air and Water permitting activities. Coordinate and } \\
\text { integrate Hanford Site Air Operating Permit activities. Perform Air and Water permitting } \\
\text { coordination and documentation consolidation for the Hanford Site as directed by DOE- } \\
\text { RL and subject to cooperation by the other contractors. Most activities are established } \\
\text { by regulatory authority and by conditions of the various air and water permits that are in } \\
\text { place on the Hanford Site. }\end{array}$} \\
\hline \multicolumn{4}{|c|}{$\begin{array}{l}\text { Deliverables to DOE-RL include recommendations when mandates or determinations } \\
\text { from regulatory entities have the potential to impact available resources or operations, } \\
\text { air and water permitting schedules, a Risk Management Plan, a revised version of the } \\
\text { Hanford Site Stormwater Pollution Prevention Plan, the annual report of operating logs } \\
\text { of portable and temporary radioactive air emission units (PTRAEU's) for CY } 1998 \text { and } \\
\text { update of the PTRAEU permit, assessment reports of PTRAEU compliance, semi- } \\
\text { annual air emission reports, annual compliance certifications for the air operating permit } \\
\text { (AOP), AOP modifications, and an AOP implementation plan. }\end{array}$} \\
\hline
\end{tabular}




\begin{tabular}{|c|c|c|c|}
\hline $\begin{array}{l}\text { 1. Dictionary title: } \\
\text { Environmental } \\
\text { Compliance } \\
\text { Program }\end{array}$ & $\begin{array}{l}\text { 2. Date: } \\
08 / 04 / 98\end{array}$ & $\begin{array}{l}\text { 3. PBS Number: } \\
\text { RL-OT01 }\end{array}$ & $\begin{array}{l}\text { 4. Dict Rev: } \\
0\end{array}$ \\
\hline $\begin{array}{l}\text { 5. WBS No: } \\
1.8 .2 .3 .5\end{array}$ & $\begin{array}{l}\text { 6. B\&R No:: } \\
\text { EWO290700 }\end{array}$ & \multicolumn{2}{|l|}{ 7. Baseline CR No: } \\
\hline \multicolumn{4}{|c|}{$\begin{array}{l}\text { 8. Organization Name: Environmental Compliance Program } \\
\text { 9. Scope of Work: }\end{array}$} \\
\hline \multicolumn{4}{|c|}{ Sire-Wide Environmental Reports (1.8.2.3.5), FDS WBS 1 R1H05 } \\
\hline \multicolumn{4}{|c|}{$\begin{array}{l}\text { Prepare documentation required for monitoring and reporting hazardous waste and } \\
\text { chemical information including reporting under Emergency Planning and Community } \\
\text { Right-To-Know Act, Resource Conservation and Recovery Act, and Toxic Substances } \\
\text { Control Act regulations, as well as the Tri-Party Agreement. Ensure compliance with } \\
\text { Hanford Site reporting requirements for dangerous and/or hazardous waste and toxic } \\
\text { substances specified by environmental regulations and statutes. Perform Environmental } \\
\text { Reporting coordination and documentation consolidation for the Hanford Site as directed } \\
\text { by DOE-RL and subject to cooperation by the other contractors. }\end{array}$} \\
\hline \multicolumn{4}{|c|}{$\begin{array}{l}\text { Preparation of specific Hanford environmental reports on a site wide basis is mandated } \\
\text { by state and federal regulations. Noncompliance with regulatory requirements is } \\
\text { grounds for substantial enforcement actions by the regulators }\end{array}$} \\
\hline \multicolumn{4}{|c|}{$\begin{array}{l}\text { Deliverables to DOE-RL include the EPCRA } 312 \text { Tier Two Emergency and Hazardous } \\
\text { Chemical Inventory Report, the EPCRA } 313 \text { Toxic Chemical Release Inventory (TRI) } \\
\text { Report, the Annual Dangerous Waste Report, the Hanford Site Annual Polychlorinated } \\
\text { Biphenyl (PCB) Document Log, the Annual PCB Report, and the Land Disposal } \\
\text { Restrictions (LDR) Report. }\end{array}$} \\
\hline
\end{tabular}




\begin{tabular}{|c|c|c|c|}
\hline $\begin{array}{l}\text { 1. Dictionary Title: } \\
\text { Environmental } \\
\text { Compliance } \\
\text { Program }\end{array}$ & $\begin{array}{l}\text { 2. Date: } \\
08 / 04 / 98\end{array}$ & $\begin{array}{l}\text { 3. PBS Number: } \\
\text { RL-OT01 }\end{array}$ & $\begin{array}{l}\text { 4. Dict Rev: } \\
0\end{array}$ \\
\hline $\begin{array}{l}\text { 5. WBS No: } \\
1.8 .2 .3 .6\end{array}$ & $\begin{array}{l}\text { 6. B\&RNO:: } \\
\text { EWO290700 }\end{array}$ & \multicolumn{2}{|c|}{ 7. Baseline CR No: } \\
\hline \multicolumn{4}{|c|}{$\begin{array}{l}\text { 8. Organization Name: Environmental Compliance Program } \\
\text { 9. Scope of Work: }\end{array}$} \\
\hline \multicolumn{4}{|c|}{ Tri-Party Agreement integration (1.8.2.3.6), FDS WBS 1R1H06: } \\
\hline \multicolumn{4}{|c|}{$\begin{array}{l}\text { Provide support to DOE-RL in coordinating and integrating the Tri-Party Agreement } \\
\text { requirements. Perform Tri-Party Agreement coordination and documentation } \\
\text { consolidation for the Hanford Site as directed by DOE-RL and subject to cooperation by } \\
\text { the other contractors. Oversee the development and maintenance of guidelines and } \\
\text { procedures necessary for implementation of the Tri-Party Agreement. Participate in } \\
\text { resolution of the technical issues related to Tri-party Agreement activities. Oversee the } \\
\text { operation and maintenance of the administrative records and public information } \\
\text { repositories required by the Tri-Party Agreement. Prepare the Tri-Party agreement } \\
\text { annual update, quarterly reports, and provide status and progress on the Tri-Party } \\
\text { Agreement milestones. Assist in the coordination of Tri-Party Agreement Public } \\
\text { Meetings, and provide regulators with direct access to TPA-related databases. }\end{array}$} \\
\hline \multicolumn{4}{|c|}{$\begin{array}{l}\text { These activities are required pursuant to the Hanford Federal Facisity Agreement and } \\
\text { Consent Order (TPA)/Community Relation Plan. }\end{array}$} \\
\hline \multicolumn{4}{|c|}{$\begin{array}{l}\text { Deliverables to DOE-RL include a weekly Tri-Party Agreement executive summary } \\
\text { report, a bi-weekly Tri-Party Agreement activities and milestones tracking report, a } \\
\text { monthly Tri-Party Agreement milestone update report and a monthly Tri-Party } \\
\text { Agreement milestone and target date status update chart. }\end{array}$} \\
\hline
\end{tabular}




\begin{tabular}{|c|c|c|c|}
\hline $\begin{array}{l}\text { 1. Dictionary Title: } \\
\text { Environmental } \\
\text { Compliance } \\
\text { Program }\end{array}$ & $\begin{array}{l}\text { 2. Date: } \\
08 / 04 / 98\end{array}$ & $\begin{array}{l}\text { 3. PBS Number: } \\
\text { RL-OT01 }\end{array}$ & $\begin{array}{l}\text { 4. Dict Rev: } \\
0\end{array}$ \\
\hline $\begin{array}{l}\text { 5. WBS No.: } \\
1.8 .2 .3 .7\end{array}$ & $\begin{array}{l}\text { 6. B\&R NO:: } \\
\text { EWO290700 }\end{array}$ & \multicolumn{2}{|c|}{ 7. Baseline CR No:: } \\
\hline \multicolumn{4}{|c|}{$\begin{array}{l}\text { 8. Organization Name: Environmental Compliance Program } \\
\text { 9. Scope of Work: }\end{array}$} \\
\hline \multicolumn{4}{|c|}{ RCRA Services (1.8.2.3.7), FDS WBS 1R IHO7: } \\
\hline \multicolumn{4}{|c|}{$\begin{array}{l}\text { Prepare crosscutting RCRA permit application documentation. Participate in } \\
\text { negotiations with federal, state, and local regulatory agencies in the establishment of } \\
\text { permitting priorities and schedules, in the resolution of permitting issues, and in the } \\
\text { development of compliance plans and agreements involving Contractor permitting } \\
\text { activities. Coordinate Hanford Facility RCRA permit activities. Perform RCRA permitting } \\
\text { coordination and documentation consolidation for the Hanford Site as directed by DOE- } \\
\text { RL and subject to cooperation by the other contractors. Maintain an administrative } \\
\text { record repository for RCRA permitting information. Maintain database systems used to } \\
\text { evaluate compliance with the Hanford Facility RCRA Permit and other applicable } \\
\text { regulations. Assist in responses to cross-cutting RCRA and TSCA compliance issues. }\end{array}$} \\
\hline \multicolumn{4}{|c|}{$\begin{array}{l}\text { Most activities are established by regulatory authority and by conditions of the Hanford } \\
\text { Facility RCRA Permit. }\end{array}$} \\
\hline \multicolumn{4}{|c|}{$\begin{array}{l}\text { Deliverables to DOE-RL include documentation to keep the General Information Portion } \\
\text { of the RCRA Permit (DOE/RL-91-28) current, a contractor-certified Part A, Form } 1 \\
\text { revision, a revised Form } 2 \text { within } 90 \text { days after receiving documentation indicating that } \\
\text { this form requires updating, the Annual Report of Hanford Facility RCRA permit } \\
\text { Noncompliance, the annual Reporting Updating Projections of Anticipated Costs for } \\
\text { Closure and Post-closure of TSD Units, and the annual Hanford Facility RCRA permit } \\
\text { Mapping information Report. }\end{array}$} \\
\hline
\end{tabular}




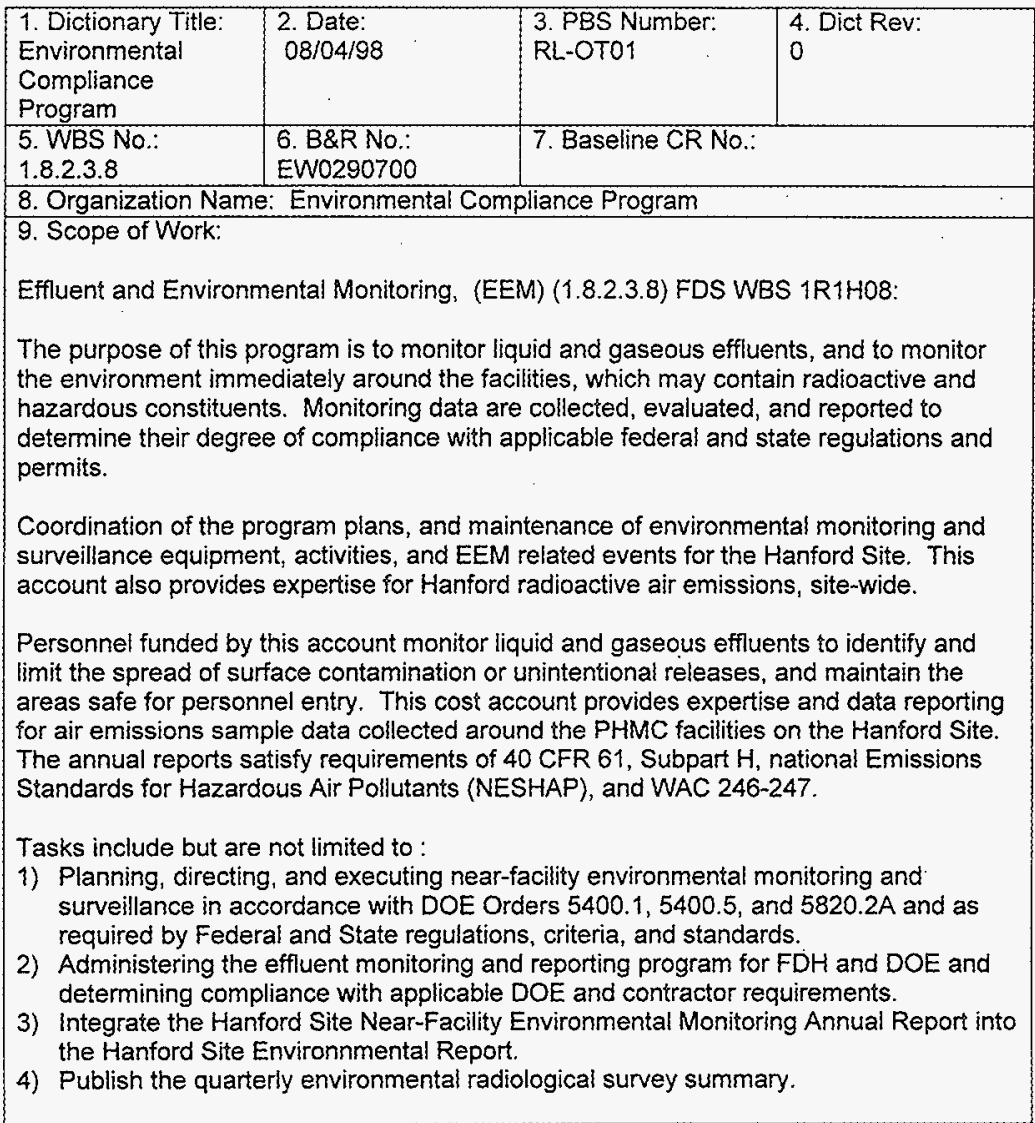




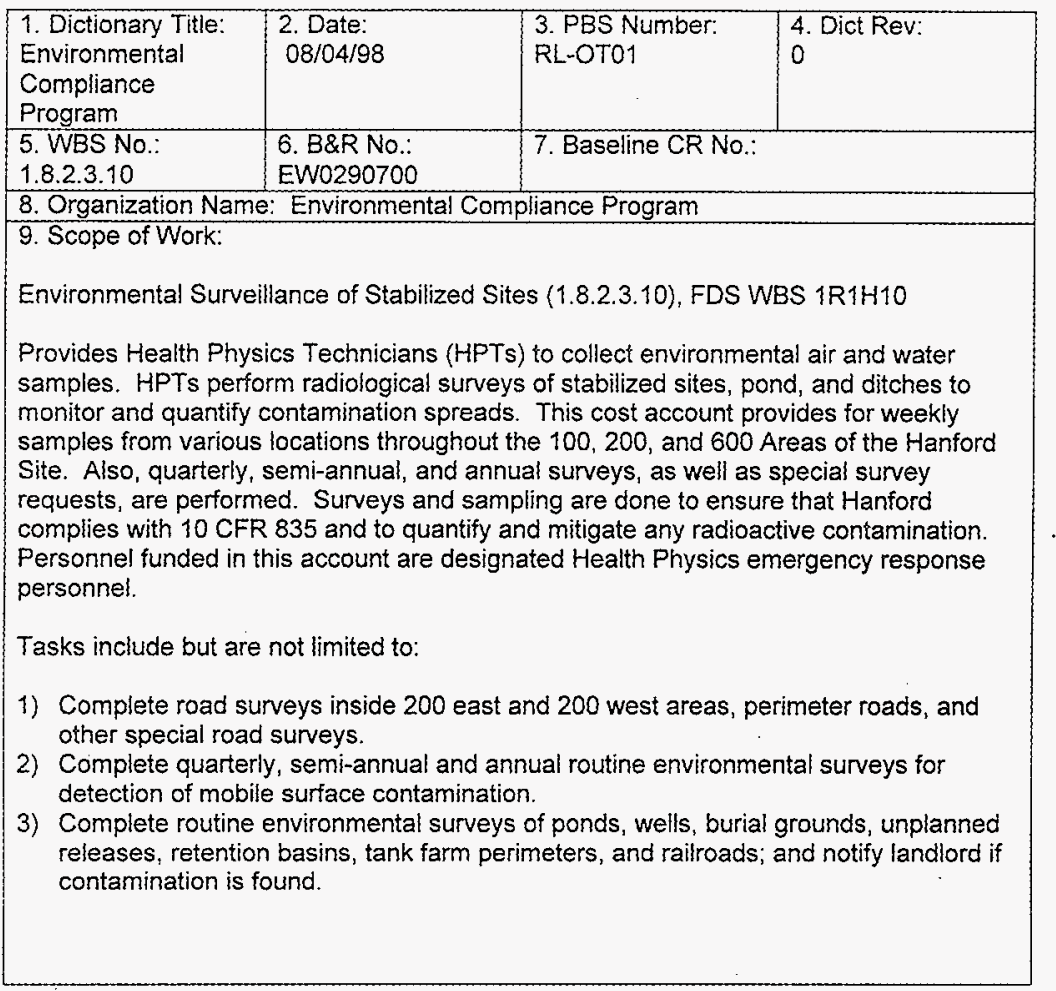




\begin{tabular}{|c|c|c|c|}
\hline $\begin{array}{l}\text { 1. Dictionary Title: } \\
\text { Environmental } \\
\text { Compliance } \\
\text { Program }\end{array}$ & $\begin{array}{l}\text { 2. Date: } \\
08 / 04 / 98\end{array}$ & $\begin{array}{l}\text { 3. PBS Number: } \\
\text { RL-OT01 }\end{array}$ & $\begin{array}{l}\text { 4. Dict Rev: } \\
0\end{array}$ \\
\hline $\begin{array}{l}\text { 5. WBS No:: } \\
1.8 .2 .3 .11 \\
\end{array}$ & $\begin{array}{l}\text { 6. B\&R No.: } \\
\text { EWO290700 }\end{array}$ & \multicolumn{2}{|c|}{ 7. Baseline CR No:: } \\
\hline \multicolumn{4}{|c|}{ 8. Organization Name: Environmental Compliance Program } \\
\hline \multicolumn{4}{|c|}{ 9. Scope of Work: } \\
\hline \multicolumn{4}{|c|}{$\begin{array}{l}\text { This project executes the design, procurement, construction, startup and turnover } \\
\text { activities for stack monitoring system upgrades on selected Tank Waste Remediation } \\
\text { System (TWRS) ventilation systems. FY } 1999 \text { objectives include definitive design, } \\
\text { procurement of material, permitting, and the initiation of construction. The technical } \\
\text { objectives for representative sampling of ventilation exhaust streams are based on } \\
\text { historical data from Hanford and other DOE sites. The project will modify existing } \\
\text { facilities and provide compliant monitoring systems for six Tank Waste Remediation } \\
\text { System (TWRS) "major" ventilation system stacks. Major stacks must meet the } \\
\text { monitoring requirements of } 40 \mathrm{CFR} 6 \text { S Subpart H. }\end{array}$} \\
\hline \multicolumn{4}{|c|}{$\begin{array}{l}\text { The six stacks requiring upgrades for compliant sampling are:296-A-25 (244-A DCRT), } \\
\text { 296-B-28 (244-BX DCRT), 296-C-5 (244-CR Vault), 296-P-16 (241-C Tank Farm), 296- } \\
\text { S-22 (244-S DCRT), and 296-T-18 (244-TX DCRT). }\end{array}$} \\
\hline \multicolumn{4}{|c|}{$\begin{array}{l}\text { For the purposes of this project, "start of construction" for all six stacks shall be defined } \\
\text { as: "place concrete pads before June } 30^{\text {th }} \text { to meet the six legally enforceable Federal } \\
\text { Facility Compliance Agreement (FFCA) milestones". }\end{array}$} \\
\hline
\end{tabular}




\begin{tabular}{|c|c|c|c|}
\hline $\begin{array}{l}\text { 1. Dictionary Title: } \\
\text { Environmental } \\
\text { Compliance } \\
\text { Program }\end{array}$ & $\begin{array}{l}\text { 2. Date: } \\
08 / 04 / 98\end{array}$ & $\begin{array}{l}\text { 3. PBS Number: } \\
\text { RL-OT01 }\end{array}$ & $\begin{array}{l}\text { 4. Dict Rev: } \\
0\end{array}$ \\
\hline $\begin{array}{l}\text { 5. WBS No.: } \\
1.8 .2 .3 .12\end{array}$ & $\begin{array}{l}\text { 6. B\&R NO:: } \\
\text { EW0290700 }\end{array}$ & \multicolumn{2}{|c|}{ 7. Baseline CR No:: } \\
\hline \multicolumn{4}{|c|}{ 8. Organization Name: Environmental Compliance Program } \\
\hline \multicolumn{4}{|c|}{ 9. Scope of Work: } \\
\hline \multicolumn{4}{|c|}{$\begin{array}{l}\text { This account provides expense funds for executing the design, procurement, } \\
\text { construction, startup and turnover activities for stack monitoring system upgrades on } \\
\text { selected Tank Waste Remediation System (TWRS) ventilation systems. FY } 1999 \\
\text { objectives include the project management and integration (other project costs) } \\
\text { supporting Project W-420 definitive design, procurement, permitting, and construction. }\end{array}$} \\
\hline
\end{tabular}




\subsection{Responsibility Assignment Matrix (RAM)}

The RAM is a matrix that identifies the responsible individual for accomplishing the work scope that is identified in the WBS dictionary section.

\begin{tabular}{|c|c|c|c|c|c|c|}
\hline RL WBS & $\begin{array}{c}\text { COST } \\
\text { ACCOUNT }\end{array}$ & CAP TITLE & CAM & $\begin{array}{l}\text { FUND } \\
\text { TYPE }\end{array}$ & $B \& R$ CODE & PBS \\
\hline 1.08 .02 .03 .01 & 1R1HO1 & Program Management & Giese, KA & $\mathrm{OE}$ & EW02J111 & OT01 \\
\hline 1.08 .02 .03 .02 & 1R1H02 & NEPA & Cummins, GD & $\mathrm{OE}$ & EW02J $\{11$ & OT01 \\
\hline 1.08 .02 .03 .03 & 1R1403 & $\begin{array}{l}\text { Compliance Integration and } \\
\text { Process Improvement }\end{array}$ & Brunke, RC & OE & EW02J111 & OTO1 \\
\hline 1.08 .02 .03 .04 & 1R1H04 & Air and Water Services & Greager, EM & $\mathrm{OE}$ & EW02J111 & OTO1 \\
\hline 1.08 .02 .03 .05 & 1R1H05 & $\begin{array}{l}\text { Site wide Environmental } \\
\text { Reports }\end{array}$ & Skolrud, JO & OE & EW02J111 & \\
\hline 1.08 .02 .03 .06 & 1R1H06 & TPA integration & Arnold, LD & $\mathrm{OE}$ & EW02J $\{11$ & OTO1 \\
\hline 1.08 .02 .03 .07 & 1R1H07 & RCRA Services & Engelmann, RH & OE & EW02J\}11 & OTO1 \\
\hline 1.08 .02 .03 .08 & 1R1408 & $\begin{array}{l}\text { Effluent and Environmental } \\
\text { Monitoring }\end{array}$ & Diediker, LP & $\mathrm{OE}$ & EW02J111 & OT01 \\
\hline 1.08 .02 .03 .09 & 1R1HO9 & NESHAP Compliance & Diediker, LP & $\mathrm{OE}$ & EWO2J111 & OTO1 \\
\hline 1.08 .02 .03 .10 & $1 \mathrm{R} 1 \mathrm{H} 10$ & $\begin{array}{l}\text { Environmental Surveillance } \\
\text { (HPT) of Stabilized Sites. }\end{array}$ & Diediker, LP & $\mathrm{OE}$ & EW02J111 & 0701 \\
\hline 1.08 .02 .03 .11 & 1R1H11 & $\begin{array}{l}\text { W-420 Stack Monitoring } \\
\text { System Upgrades }\end{array}$ & Carpenter, KE & GPP & EW02J111 & OT01 \\
\hline 1.08 .02 .03 .12 & 1R1H12 & $\begin{array}{l}\text { Other Project Activities in } \\
\text { Support of Project W- } 420\end{array}$ & Carpenter, KE & $O E$ & EW02J111 & OTO1 \\
\hline
\end{tabular}




\subsection{Schedule Baseline}

\subsection{Project Master Baseline Schedule (PMBS)}

The PMBS covers the life of the project. Since many of the Environmental Compliance Program milestone deliverables are annual deliverables, the schedule displays the lifecycle of each cost account only. A summary description of the out-year milestones can be found in section 3.5, "Milestone Description Sheets - Out-years". Please see the figure on the following page. 


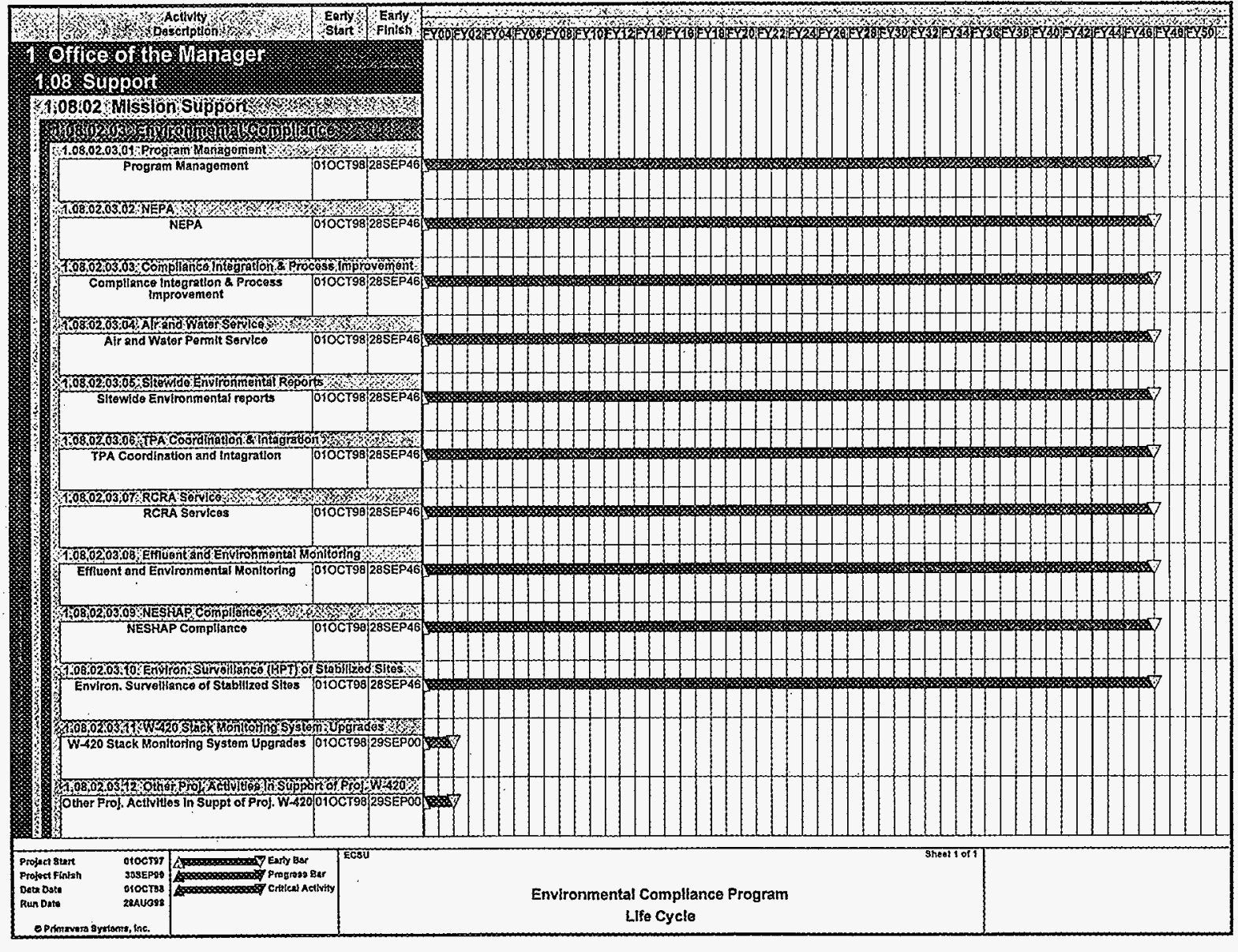




\section{HNF-SP-1112, Rev. 4}

\subsection{Execution Year PMBS}

The Execution Year PMBS displays one year out of the PMBS. This schedule contains enforceable agreement milestones and other milestones at both the DOE-HQ and RL level. Please see the figure on the following page. 


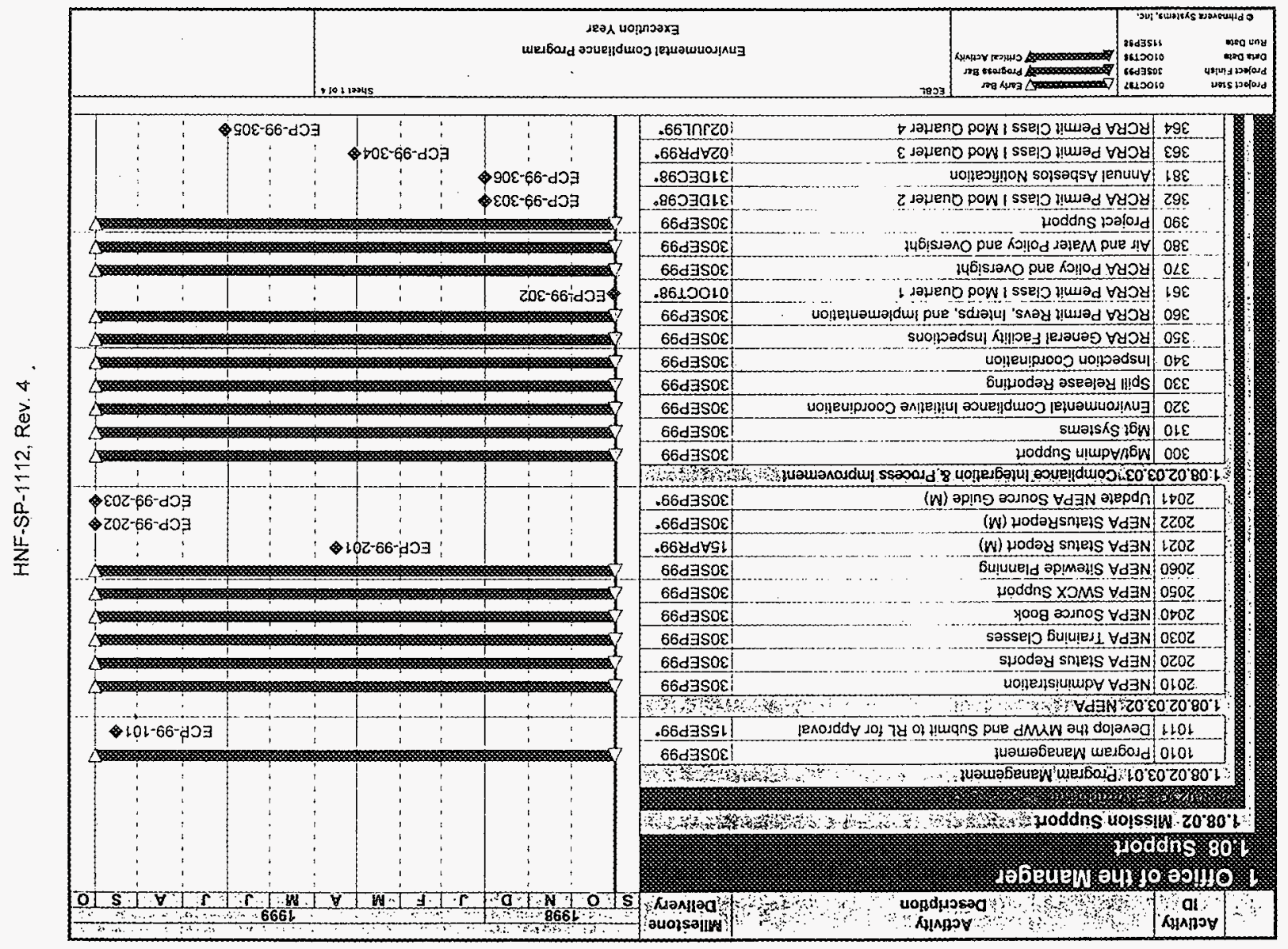




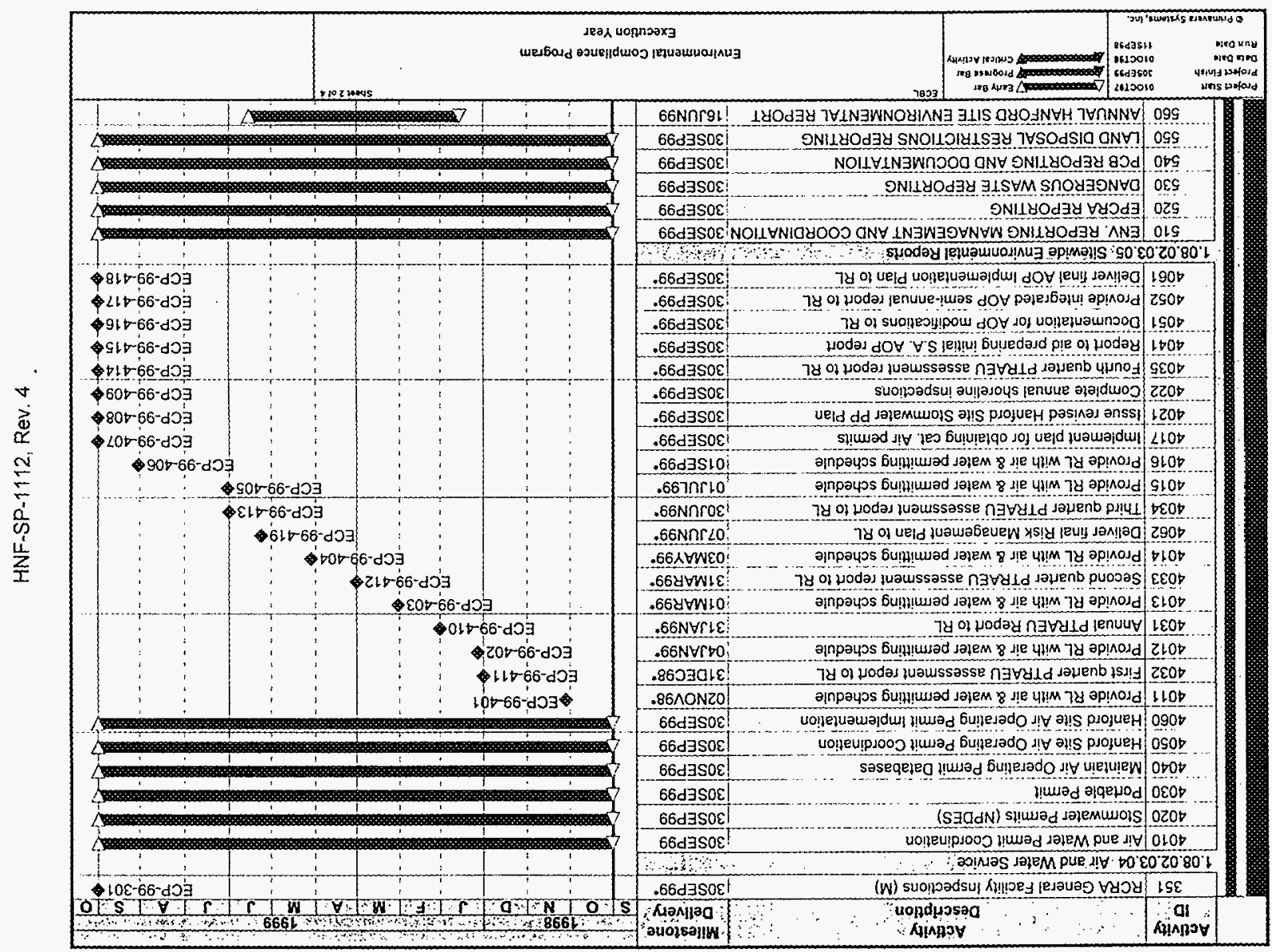




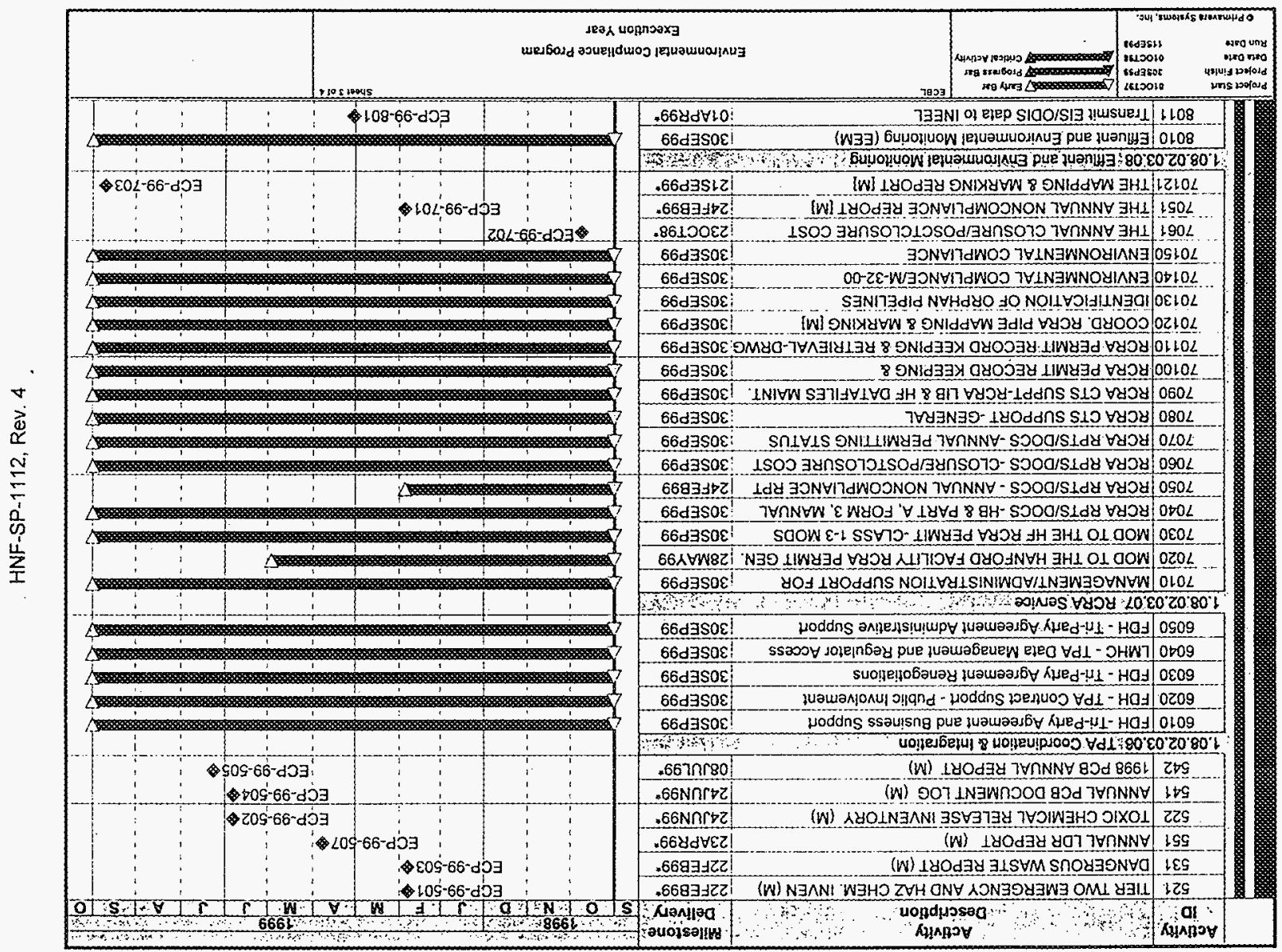




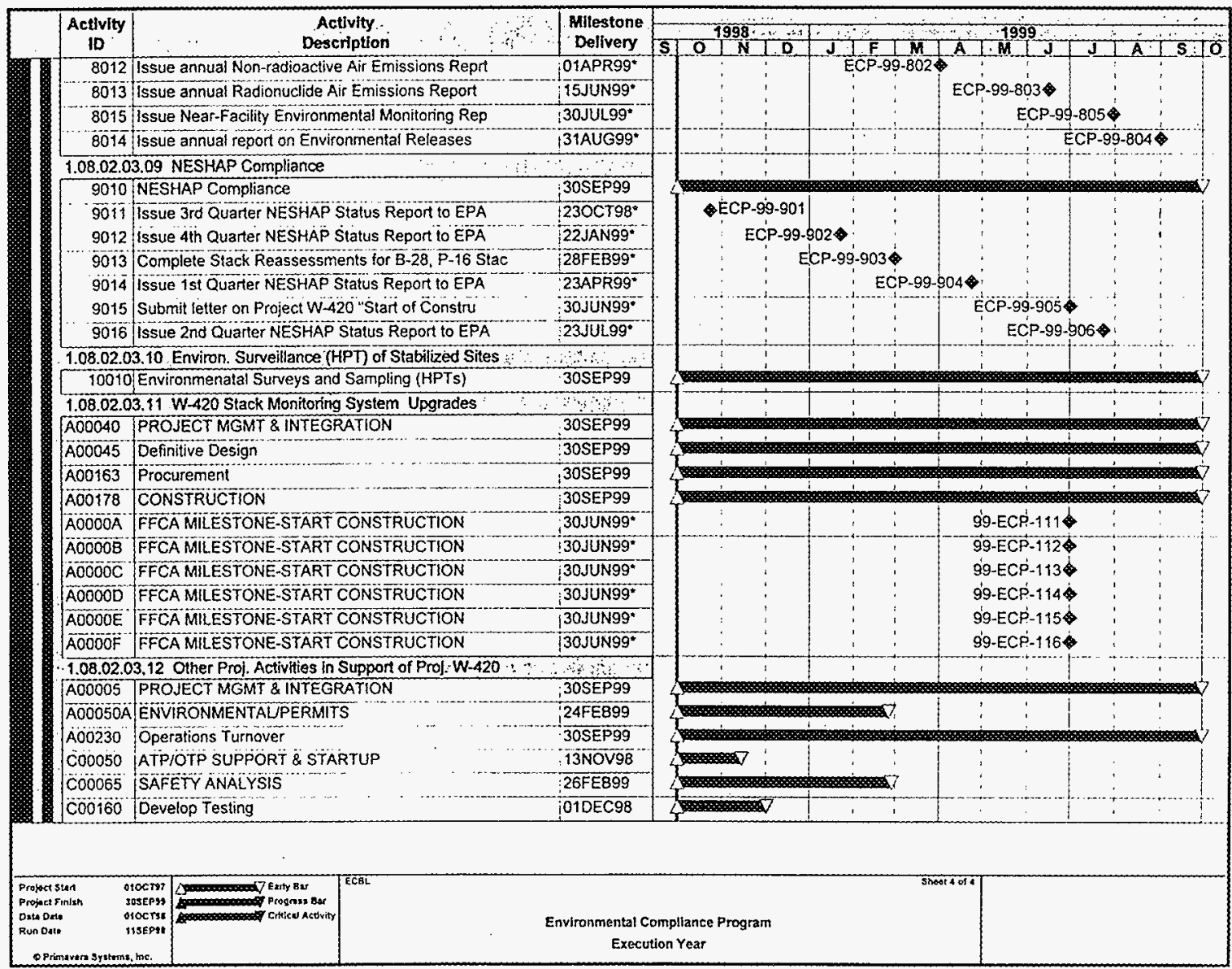


HNF-SP-1112, Rev. 4

\subsection{Milestones}

The milestone list is housed in the Central Milestone Module (CMM). The list includes all "Enforceable Agreement" - type Milestones, which are HQ level and all "Other" - type milestones, which are RL-level. The milestones marked with an asterisk "*" are single occurring events. All other milestones are repetitive. Please see the list on the following page. 


\begin{tabular}{|c|c|c|c|c|c|c|c|c|c|c|}
\hline \multirow{3}{*}{$\begin{array}{l}\text { MILESTONE } \\
\text { CONTROL \# }\end{array}$} & \multirow{3}{*}{$\begin{array}{l}\text { TPA-MS } \\
\text { NUNGER }\end{array}$} & \multirow{3}{*}{$\begin{array}{l}\text { TPA } \\
\text { TYPE }\end{array}$} & \multirow[b]{2}{*}{ MS } & \multirow[b]{3}{*}{ MS TITLE } & \multirow[b]{3}{*}{ TYPE } & \multicolumn{2}{|c|}{-.....-DATES......... } & \multirow[b]{3}{*}{ DNFSB } & \multirow[b]{3}{*}{ TIP } & \multirow[b]{3}{*}{ PBS } \\
\hline & & & & & & PLARNED & TPA/DNPSB & & & \\
\hline & & & i.evec & & & BASELINE & Congit & & & \\
\hline 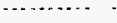 & n.............. & .... & … & (n) & $\cdots$ & .......... & .... & ..... & -.. & ....... \\
\hline ECP-99-101 & & & RI. & FY 2000 MULTI-YEAR WORK PLAN & OTH & $9 / 15 / 99$ & & n & $\mathbf{N}$ & $\mathrm{RL}-\mathrm{OTOA}$ \\
\hline$E C P-99-203$ & & & RL & MIDYEAR STATUS ON NEPA ACTIVITLES & ors & $4 / 15 / 99$ & & $n$ & $\mathrm{~N}$ & RL-OTO2 \\
\hline ECP-99-202 & & & Re & END OF YEAR STAYUS ON NEPA ACTRVITIES & OTR & $9 / 30 / 99$ & & $\mathbf{n}$ & $\mathrm{n}$ & RL-OTO2 \\
\hline ECP-99-203 & & & Ri & ISSUE UPDATED NEPA SOURCE GUIDE & отя & $9 / 30 / 99$ & & N & $n$ & RL-OT02 \\
\hline ECP-99-301 & & & 110 & RCRA GENERAL FACILITY INSPECTIONS & EA & $9 / 30 / 99$ & & м & N & $\mathrm{RL}-0 \mathrm{OTON}$ \\
\hline ECP-99-302 & & & 10 & RCRA PERMIT CLSS I MODIFICATION NOTIFICATION - QUARTER 1 & EA. & $10 / 01 / 98$ & & s & $\mathrm{N}$ & Rz-orạ \\
\hline ECP-99-305 & & & $\mathrm{HQ}$ & RCRA PERMIT CLASS I MODIEICATION BOTIFICATION - QUARTER 4 & ER & $7 / 02 / 99$ & & $\mathrm{~N}$ & v & RL-OTO? \\
\hline ECP-99-304 & & & HQ & RCRA PERMIT CLASS Y MODIEFCATIOA NOTIFICATION - QUARTER 3 & EA & $4 / 02 / 99$ & & $\mathrm{~N}$ & N & RL-OTO2 \\
\hline ECP $-99-303$ & & & $\mathrm{HC}$ & RCRA PERMIT CLASS 3 MODIPICATION NOTIFICATION - QUARTER 2 & EA & $1 / 03 / 99$ & & $n$ & n & RL-OTO: \\
\hline ECP-99-306 & & & HQ & AMUAL ASBESTOS NOTIFICATION OF IMTENT & EA & $12 / 32 / 98$ & & $\mathrm{~N}$ & 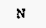 & RL-OTA: \\
\hline$E C P-99-401$ & & & $R \tau_{1}$ & PROVIDE RL WITH AIR E HATER PERMITTING SCHEDULE & OTH & $12 / 02 / 98$ & & $\mathrm{~N}$ & $\mathrm{~N}$ & $\mathrm{RL}-\mathrm{OTO2}$ \\
\hline ECP-99-402 & & & RL & PROVIDE RL WITH AIR AND KATER PERMITTING SCHEDULE & отн & $1 / 04 / 99$ & & $\mathrm{~N}$ & N & RL-Oro1 \\
\hline ECP-99-403 & & & s. & PROVIDE RL WITH ATR AND WATER PERMITTING SCHEDTHE & OTH & $3 / 0 x / 99$ & & $N$ & N & RL-OTo1 \\
\hline ECP.99-404 & & & XL & PROVIDE RE WITH AIR AND HATER PERMYTTIKG SCHEDIRE & OTH & $5 / 03 / 99$ & & $n$ & $\mathrm{~N}$ & RL=OTOL \\
\hline$E C P-99-405$ & & & RE & PROVIDE RL KITH AIR ARD HATER PERMITTING SCHEDULE & OTH & $7 / 02 / 99$ & & N & $\mathrm{H}$ & R2-orol \\
\hline ECP-99-406 & & & $\mathrm{RL}$ & PROVIDE RL WITH ATR ANO WATER PERMITTING SCHEDURE & ors & $9 / 01 / 99$ & & $\mathrm{~N}$ & N & RL-0T02 \\
\hline$E C P-99-407$ & & & RL & IMPLEMENT PLAR FOR OETAINING CATEGORICAL AIR PERMITS & OTH & $9 / 30 / 99$ & & $\mathrm{~N}$ & $\mathbf{N}$ & RL-0T01 \\
\hline ECP-99-\$09 & & & RL & ISSUE REVISED \& UPDATED KANFORD SITE STORMMATER POLUU. PREV PLAN & OTH & $9 / 30 / 99$ & & $\mathrm{~N}$ & N & $R L-O T O H$ \\
\hline ECP-99-409 & & & Ho & COMPLETE SHORELINE INSPECTIONS & EA & $9 / 30 / 99$ & & $\mathrm{~N}$ & $\mathrm{~N}$ & RL-ora1 \\
\hline ECP-99-410 & & & HO & ANNUAL PTRAEU REPORT TO DOE-KL & EA & $1 / 31 / 99$ & & N & 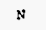 & RL-orol \\
\hline ECP-99-411 & & & RL & FIRST QUARTER PTRAEU ASSESSMEYT REPORT TO DOE-RL & ors & $12 / 31 / 98$ & & $\mathrm{~N}$ & $\mathrm{~N}$ & RL-oro2 \\
\hline ECP-99-112 & & & RL & SECONO QUARTER PTRAEU ASSESSMENT REPCRT TO DOE-RL & OTH & $3 / 31 / 99$ & & $\mathrm{~N}$ & N & RL-0T02 \\
\hline ECP-99-413 & & & RE & THIRD QUARTER PTRAEU ASSESSMENT REPORT TO DOE-RE & OTH & $6 / 30 / 99$ & & $\mathrm{~N}$ & N & RL-OTO2 \\
\hline ECP-99-414 & & & $\mathrm{RC}$ & FOURTH GUARTER PTRAEU ASSESSMENT REPORT TO DOE-RL & OTH & $9 / 30 / 99$ & & $\mathrm{~N}$ & * & RL-OTO1 \\
\hline ECP-99-425 & & & $\mathrm{RL}$ & REPORT IN PREPARATYON OF THE INITTIAL AOR SEMI-ANMUALL REPORT & OTH & $9 / 30 / 99$ & & $\mathrm{~N}$ & s & RL-OTol \\
\hline $\operatorname{ECP}-99-426$ & & & RL & INTEGRATED PHAC APPLTCATION TO MODIFY AIR OPERATING PERMIT & ors & $9 / 30 / 99$ & & $\mathrm{~N}$ & $\mathrm{~N}$ & RL-OTO2 \\
\hline ECP-99-417 & & & HO & TNTEGRATED AIR OPERATINO PERMIT SEMIARNUAL REPORT & EA & $9 / 30 / 99$ & & $N$ & $\mathrm{~N}$ & RL-OTOI \\
\hline ECP- $99-418 *$ & & & RL & FINAL AOP IMPLEMENTATION PLAN & OTH & $9 / 30 / 99$ & & $N$ & N & $\mathrm{RL}-\mathrm{OTOH}$ \\
\hline ECP-99-419* & & & $\because Q$ & DELIVER FINAL RISK MANAGEMENS FLAN TO DOR-RL & EA & $6 / 07 / 99$ & & $\mathrm{~N}$ & $\mathrm{~N}$ & RL- -0 To2 \\
\hline $8 C P .99 .501$ & & & HO & EPCRA 312 TIER TWO EMERGENCY W HAZ CHEMICAL INVENTORY REPORT & EA & $2 / 22 / 99$ & & $\mathrm{~N}$ & $\mathrm{~N}$ & RL-OTOS \\
\hline$E C P=99-502$ & & & no & EPCRA 313 TOXIC CHEMICAI, RELEASE INVEMTORY REPORT & EA & $6 / 24 / 99$ & & N & N & RL-OTQI \\
\hline ECP-99-503 & & & 10 & 1999 KANFORD SITE ANNUAL DANGEROUS KASTE REPORT & EA & $2 / 22 / 99$ & & N & a & RL-OT01 \\
\hline ECP-99-504 & & & 110 & 1998 HANFORD SITE ANAUAE POLYCHIORINATED BIPHENS DOCTMENT LOG & EA & $6 / 24 / 99$ & & $\mathrm{~N}$ & s & RL-OTO2 \\
\hline ECF-99-505 & & & tro & 1998 ANNUAL FOLYCHLORINATED BIPHENYL REPORT & EA & $7 / 08 / 99$ & & $\mathrm{~N}$ & $\mathrm{~s}$ & RL-OTO2 \\
\hline ECP-99-50? & & & HO & STGEMTT AN ANMUAL HANEORD LAMD DISPOSAL RESTRICTIONS REPORT & EA & $4 / 23 / 99$ & & $\mathrm{n}$ & $\mathrm{N}$ & RL-OTOI \\
\hline
\end{tabular}




\begin{tabular}{|c|c|c|c|c|c|c|c|c|c|c|}
\hline \multirow{3}{*}{$\begin{array}{l}\text { MILESTONE } \\
\text { CONTROL \# }\end{array}$} & \multirow{3}{*}{$\begin{array}{l}\text { TPA-MS } \\
\text { MMEER }\end{array}$} & \multirow{3}{*}{$\begin{array}{l}\text { TPA } \\
\text { TYPE }\end{array}$} & \multirow[b]{2}{*}{ MS } & \multirow[b]{3}{*}{ MS TITLE } & \multirow[b]{3}{*}{ TYPE } & \multicolumn{2}{|c|}{ - DATES-......... } & \multirow[b]{3}{*}{ DNESB } & \multirow[b]{3}{*}{ TIP } & \multirow[b]{3}{*}{ PBS \# } \\
\hline & & & & & & PLANRED & TPA/DNFSB & & & \\
\hline & & & LEVEL, & & & EASELINE & COMMit & & & \\
\hline n.t.enc.... & ........... & & $\cdots$ & 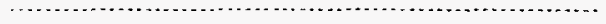 &.-- & n........ & ......... & ..... & $\cdots$ & -....... \\
\hline $8 C P-99-701$ & & & HQ & ANNUAL REPORT OF HANFORD FACILITY RCRA PERMIT NONCOMPLIANCES & EA & $2 / 24 / 99$ & & $\mathrm{~N}$ & $\mathrm{~N}$ & RL-OTO1 \\
\hline ECP-99-702 & & & HQ & UPDATE ESTIMATE OP CLOSURE AND POST-CLOSURE COSTS & ER & $10 / 23 / 98$ & & $\mathrm{~N}$ & $\mathrm{~N}$ & RL-OT01 \\
\hline$E C P-99-703$ & & & HO & COORDINATE RCRA PIPE MAPEING ATD MRRKING & $\mathrm{ER}$ & $9 / 21 / 99$ & & $\mathrm{~N}$ & $\mathrm{~N}$ & Rt,- $-\infty \mathrm{rra}_{3}$ \\
\hline ECP-99-801 & & & HO & TRANSMIT EIS/ODTS DATA TO INEEL & $8 \mathbf{2}$ & $8 / 01 / 99$ & & $\mathrm{~N}$ & N & $R L=0$ Tro \\
\hline ECP.99-802 & & & HQ & ISSUE ANWUAL NONRADTOACTIVE AIRBORTE EMISSIONS REPORT & EA & $4 / 02 / 99$ & & $\mathrm{~N}$ & $\mathrm{~N}$ & RL-OT01 \\
\hline ECP-99-803 & & & HQ & ISSUE ANMUAL RADIONUCLLIDE AIR EMISSIONS REPORT & EA & $6 / 15 / 99$ & & $\mathrm{~N}$ & $\mathrm{~N}$ & RL-OTO1 \\
\hline Ece. $99-805$ & & & Ri & ISSUE REPORT OF NEAR-FACILITY ENIRONYENTAL MONITORING & OTH & $7 / 30 / 99$ & & $\mathrm{~N}$ & $\mathrm{~N}$ & RE-OTo1 \\
\hline ECr.99-804 & & & 110 & ISSUE ANNUAL REPORT ON ENVIRONUETTAL RELEASES & EA & $8 / 31 / 99$ & & N & $\mathrm{N}$ & Rt,-orbs \\
\hline ECP.99-901 & & & HQ & ISSUE JRD QUARTER NESHRP STATUS & EA & $10 / 23 / 98$ & & $\mathrm{~N}$ & $\mathrm{~N}$ & RL-orór \\
\hline ECP.99-902 & & & HQ & ISSUE 4TH OUARTER NESHAP STATUS & EA & $1 / 22 / 99$ & & $\mathrm{~N}$ & $\mathrm{~N}$ & RL-OTO1 \\
\hline Eсp-99-903 & & & HO & COMPLETE STACK REASSESSNENSS FOR B-28, P16 STACKS & EA & $2 / 28 / 99$ & & $\mathrm{~N}$ & $\mathrm{~N}$ & RL-OTO1 \\
\hline $8 C 8 \cdot 99-904$ & & & Ho & ISSUE 1ST QUARTER RESHAP STATUS REPORT & EA & $4 / 23 / 99$ & & $\mathbf{N}$ & $n$ & RL-OTO1 \\
\hline ECP.99-905* & & & Ho & SUBMIT LETTER ON PROJECT W-420 START OF CONSTRUCTION & $\mathrm{EA}$ & $6 / 30 / 99$ & & $n$ & $\mathrm{~N}$ & RL-OTO2 \\
\hline ECP-99-906 & & & HQ & ISSTE 2ND QUARTER NESHAP STAYUS & EA & $7 / 23 / 99$ & & N & n & RL-OTOI \\
\hline ECP-99-121 $\star$ & & & Hо & CONCRETE PAD FOR STACK 296-A-25 (294-A DCRT) & EA & $6 / 30 / 99$ & & $\mathrm{~N}$ & $\mathrm{~N}$ & RL-OT01 \\
\hline ECP-99.312* & & & Ho & CONCRETE PAD FOR STACK $296-8-28$ (244-BX DCRT) & $E A$ & $6 / 30 / 99$ & & $\mathbf{N}$ & N & RL, - OTOA \\
\hline ECP-99-113* & & & HO & CONCRETE PAD FOR STACK $296-C-5$ (244-CR VAULT) & EA & $6 / 30 / 99$ & & $\mathrm{~N}$ & $\mathrm{~N}$ & RL-OTO1 \\
\hline ECP- $99-214$ * & & & HQ & CONCRETE FAO FOR STACK $296-P-16$ (244-C TANK FADM) & EA & $6 / 30 / 99$ & & $\mathrm{~N}$ & $\mathrm{~N}$ & RL-OTO: \\
\hline ECP-99-115* & & & HQ & CONCRETE PAD FOR STACK $296-5-22$ (244-S DCRT) & ER & $6 / 30 / 99$ & & N & s & RL OTOX \\
\hline ECP-99-116* & & & HQ & CONCRETE PAD FOR STACK $296-T-18$ (244-TX DCRT) & EA & $5 / 30 / 99$ & & N & $\mathrm{N}$ & RL-OTO1 \\
\hline
\end{tabular}


HNF-SP-1112, Rev. 4

\subsection{Milestone Description Sheets - Execution Year}

The milestone description sheets are housed in the CMM (Central Milestone Module) database. A milestone description sheet is provided for all EA-type (enforceable agreement) and Other-type milestones for the FY99 execution year. All EA milestones are HQ-level, while the Other milestones are RL-level. Please see the MDS on the following pages. 
PHMC

MILESTONE DESCRIPTION SHEET

\begin{tabular}{|c|c|c|c|c|}
\hline \multicolumn{4}{|c|}{ Title: FY 2000 MULTI-YEAR WORK PLAN } & Date: $8 / 04 / 98$ \\
\hline \multicolumn{4}{|c|}{ Assigned To: W. D. ADAIR } & CIN: \\
\hline \multicolumn{4}{|c|}{ Program WBS Designator: $1 \cdot 8 \cdot 2.3 .1$} & Due Date: $9 / 15 / 99$ \\
\hline \multicolumn{5}{|c|}{ PBS No: RL-0T01 } \\
\hline \multicolumn{2}{|c|}{ MC \#: ECP-99-101 } & TPA No: & & Rev: 0 \\
\hline $\begin{array}{l}\text { MILESTONE } \\
\text { LEVEL: } \\
\text { DOE-HQ } \\
\times \text { DOE-RL } \\
\text { DOE-FO } \\
\text { CONTRACTOR }\end{array}$ & $\begin{array}{l}\text { MILESTONE } \\
\text { TYPE: } \\
\text { EA } \\
\text { PEG } \\
\times \text { OTHER } \\
\text { TIP }\end{array}$ & $\begin{array}{l}\text { DNFSB STATUS: } \\
\text { DNFSB }(Y / N): N \\
\text { COMMIT \#: } \\
\text { RECOMM \#: }\end{array}$ & $\begin{array}{l}\text { DELIVERABLE: } \\
\text { Report } \\
\text { Letter } \\
\text { Drawing(s) } \\
\text { X Other (Specify) } \\
\text { DOCUMENT }\end{array}$ & $\begin{array}{l}\text { ADDRESS TO: } \\
\text { DOE-HO } \\
\times \text { OOE-RL } \\
\text { Other (Specify) }\end{array}$ \\
\hline \multicolumn{5}{|c|}{$\begin{array}{l}\text { Milestone Description: } \\
\text { The FY } 2000 \text { MYWP document provides the mechanism to define the cost, } \\
\text { schedule, and technical aspects of the work to be performed in the. } \\
\text { Environmental Compliance Program. The MYWP is a baseline control document. } \\
\text { Therefore adjustments to cost. schedule, or technical workscope will be } \\
\text { made by change control. }\end{array}$} \\
\hline \multicolumn{5}{|c|}{$\begin{array}{l}\text { Description of what constitutes completion of this milestone: } \\
\text { Submit the final FY } 2000 \text { MYWP to DOE-RL by September 15, 1999. The MYWP at } \\
\text { this time has undergone several reviewS with DOE-RL and should reflect the } \\
\text { fina? version. Final signoff to occur by September } 30 \text {, 1999. }\end{array}$} \\
\hline
\end{tabular}


HNF-SP-1112, ReV. 4

\section{PHMC \\ MILESTONE DESCRIPTION SHEET}

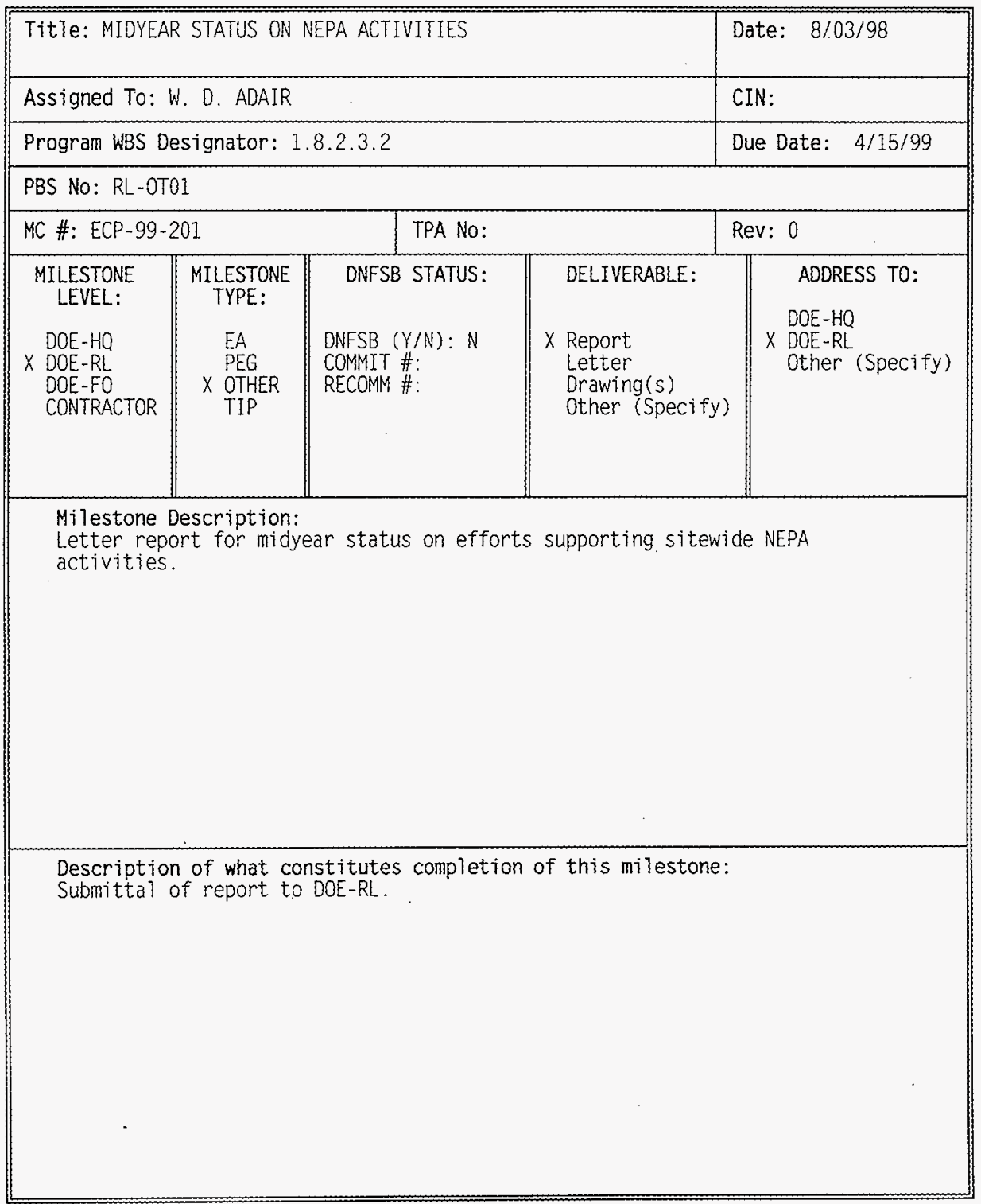




\section{PHMC}

MILESTONE DESCRIPTION SHEET

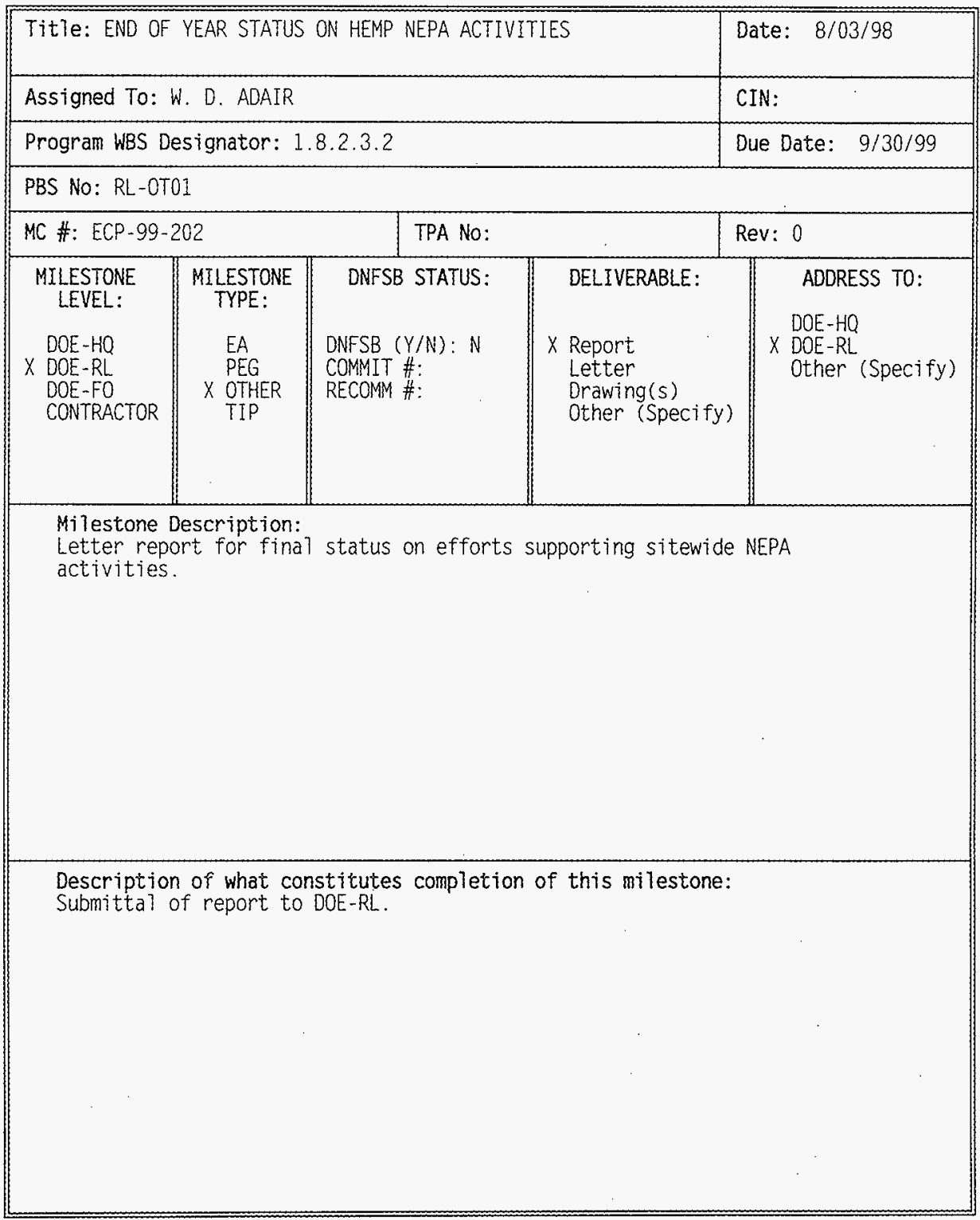




\section{PHMC \\ MILESTONE DESCRIPTION SHEET}

\begin{tabular}{|c|c|c|c|c|}
\hline \multicolumn{4}{|c|}{ Title: ISSUE UPDATED NEPA SOURCE GUIDE } & Date: $8 / 11 / 98$ \\
\hline \multicolumn{4}{|c|}{ Assigned To: W. D. ADAIR } & CIN: \\
\hline \multicolumn{4}{|c|}{ Program WBS Designator: 1.8 .2 .3 .2} & Due Date: $9 / 30 / 99$ \\
\hline \multicolumn{5}{|c|}{ PBS NO: RL-OTOI } \\
\hline \multicolumn{2}{|c|}{ MC \#: ECP-99-203 } & TPA No: & & Rev: 0 \\
\hline $\begin{array}{l}\text { MILESTONE } \\
\text { LEVEL: } \\
\\
\text { DOE-HQ } \\
\times \text { DOE-RL } \\
\text { DOE-FO } \\
\text { CONTRACTOR }\end{array}$ & $\begin{array}{l}\text { MILESTONE } \\
\text { TYPE: } \\
\text { EA } \\
\text { PEG } \\
\times \text { OTHER } \\
\text { TIP }\end{array}$ & $\begin{array}{l}\text { DNFSB STATUS: } \\
\text { DNFSB }(Y / N): N \\
\text { COMMIT \#: } \\
\text { RECOMM \#: }\end{array}$ & $\begin{array}{l}\text { OELIVERABLE: } \\
\text { X Report } \\
\text { Letter } \\
\text { Orawing(s) } \\
\text { Other (Specify) }\end{array}$ & $\begin{array}{l}\text { ADDRESS TO: } \\
\text { OOE-HQ } \\
\times \text { DOE-RL } \\
\text { Other (Specify) }\end{array}$ \\
\hline \multicolumn{5}{|c|}{$\begin{array}{l}\text { Milestone Description: } \\
\text { Update NEPA Source Guide information and issue revised reference. Place } \\
\text { Source Guide information on Hanford Intranet. }\end{array}$} \\
\hline \multicolumn{5}{|c|}{$\begin{array}{l}\text { Description of what constitutes completion of this milestone: } \\
\text { Submittal of updated NEPA Source Guide to DOE-RL. }\end{array}$} \\
\hline
\end{tabular}


PHMC

\section{MILESTONE DESCRIPTION SHEET}

\begin{tabular}{|c|c|c|c|c|}
\hline \multicolumn{4}{|c|}{ Tit7e: RCRA GENERAL FACILITY INSPECTIONS } & Date: $8 / 10 / 98$ \\
\hline \multicolumn{4}{|c|}{ Assigned To: W. D. ADAIR } & CIN: \\
\hline \multicolumn{4}{|c|}{ Program WBS Designator: 1.8.2.3.3 } & Due Date: $9 / 30 / 99$ \\
\hline \multicolumn{5}{|c|}{ PBS No: RL-OT01 } \\
\hline \multicolumn{2}{|c|}{ MC \#: ECP-99-301 } & TPA No: & & Rev: 0 \\
\hline $\begin{array}{l}\text { MILESTONE } \\
\text { LEVEL: } \\
\text { X DOE-HO } \\
\text { DOE-RL } \\
\text { DOE-FO } \\
\text { CONTRACTOR }\end{array}$ & $\begin{array}{l}\text { MILESTONE } \\
\text { TYPE: } \\
\text { X EA } \\
\text { PEG } \\
\text { OTHER } \\
\text { TIP }\end{array}$ & $\begin{array}{l}\text { DNFSB STATUS: } \\
\text { ONFSB }(Y / N): N \\
\text { COMMIT \#: } \\
\text { RECOMM \#: }\end{array}$ & $\begin{array}{l}\text { DELIVERABLE: } \\
\text { Report } \\
\text { Letter } \\
\text { Drawing(s) } \\
\text { X Other (Specify) } \\
\text { INSPECTIONS }\end{array}$ & $\begin{array}{l}\text { ADDRESS TO: } \\
\text { DOE-HQ } \\
\times \text { DOE-RL } \\
\text { Other (Specify) }\end{array}$ \\
\hline \multicolumn{5}{|c|}{$\begin{array}{l}\text { Milestone Description: } \\
\text { The } 100,200.300 .400 \text {. and } 1100 \text { areas will annually be visually inspected } \\
\text { for malfunctions, deteriorations, operator errors, and discharges that } \\
\text { could cause or lead to the release of dangerous waste constituents to the } \\
\text { environment or that could threat human life. Twice per year, the Columbia } \\
\text { River banks within the Hanford boundaries will be inspected, once at the } \\
\text { low water mark of the year and once as determined by the permittees. The } \\
\text { requirement is contained in Condition II. O. of the Hanford Facility RCRA } \\
\text { Permit (Dangerous Waste Portion). Per Condition II. } 0 \text {. inspection } \\
\text { notifications wi7l be provided to DoE-RL. for transmittal to Ecology, at } \\
\text { least seven days before the inspections are conducted. The milestone is. } \\
\text { complete by placing an arnual inspection report in the Hanford Facility } \\
\text { Operating Record. Genera? Information File. }\end{array}$} \\
\hline \multicolumn{5}{|c|}{$\begin{array}{l}\text { Description of what constitutes completion of this milestone: } \\
\text { The } 100,200 \text {. } 200 \mathrm{~W} \text {. } 300 \text {, } 400 \text {, and } 1100 \text { areas wi } 11 \text { be inspected annually, } \\
\text { and the banks of the Columbia River within the boundaries of the Hanford } \\
\text { Site will be inspected twice per year. }\end{array}$} \\
\hline
\end{tabular}




\section{PHMC \\ MILESTONE DESCRIPTION SHEET}

\begin{tabular}{|c|c|c|c|c|}
\hline \multicolumn{4}{|c|}{$\begin{array}{r}\text { Title: RCRA PERMIT CLASS I MODIFICATION } \\
\text { NOTIFICATION - QUARTER } 1\end{array}$} & Date: $8 / 10 / 98$ \\
\hline \multicolumn{4}{|c|}{ Assigned To: W. D. ADAIR } & CIN: \\
\hline \multicolumn{4}{|c|}{ Program WBS Designator: 1.8.2.3.3 } & Due Date: $10 / 01 / 98$ \\
\hline \multicolumn{5}{|c|}{ PBS No: RL-0T01 } \\
\hline \multicolumn{2}{|c|}{ MC \#: ECP-99-302 } & TPA No: & & Rev: 0 \\
\hline $\begin{array}{l}\text { MILESTONE } \\
\text { LEVEL: } \\
\times \text { DOE-HQ } \\
\text { DOE-RL } \\
\text { DOE-FO } \\
\text { CONTRACTOR }\end{array}$ & $\begin{array}{l}\text { MILESTONE } \\
\text { TYPE: } \\
\text { X EA } \\
\text { PEG } \\
\text { OTHER } \\
\text { TIP }\end{array}$ & $\begin{array}{l}\text { DNFSB STATUS: } \\
\text { DNFSB }(Y / N): N \\
\text { COMMIT \#: } \\
\text { RECOMM \#: }\end{array}$ & $\begin{array}{l}\text { DELIVERABLE: } \\
\times \text { Report } \\
\text { Letter } \\
\text { Orawing(s) } \\
\text { Other (Specify) }\end{array}$ & $\begin{array}{l}\text { AODRESS TO: } \\
\text { DOE-HQ } \\
\times \text { DOE-RL } \\
\text { Other (Specify) }\end{array}$ \\
\hline \multicolumn{5}{|c|}{$\begin{array}{l}\text { Milestone Description: } \\
\text { A quarterly notification report wi17 be submitted to DOE-RL as required by } \\
\text { WAC } 173-303-830(4)(a)(i)(A) \text { and Condition I.C. } 3 \text { of the Hanford Facility } \\
\text { RCRA Permit (Dangerous Waste Portion). The report is to provide } \\
\text { information for all Class I Modifications put into effect at Contractor Co- } \\
\text { Operated TSO units during the reporting period. The report will include } \\
\text { TSD unit information received in a complete form } 30 \text { days before the } \\
\text { regulator due date. }\end{array}$} \\
\hline \multicolumn{5}{|c|}{$\begin{array}{l}\text { Description of what constitutes completion of this milestone: } \\
\text { Submit quarterly Notification of Class I Modifications of the Hanford } \\
\text { Facility RCRA Permit to DOE-RL by October 1, } 1998 \text { for transmittal by DOE-RL } \\
\text { to Ecology by October } 9.1998 \text {. }\end{array}$} \\
\hline
\end{tabular}




\section{PHMC \\ MILESTONE DESCRIPTION SHEET}

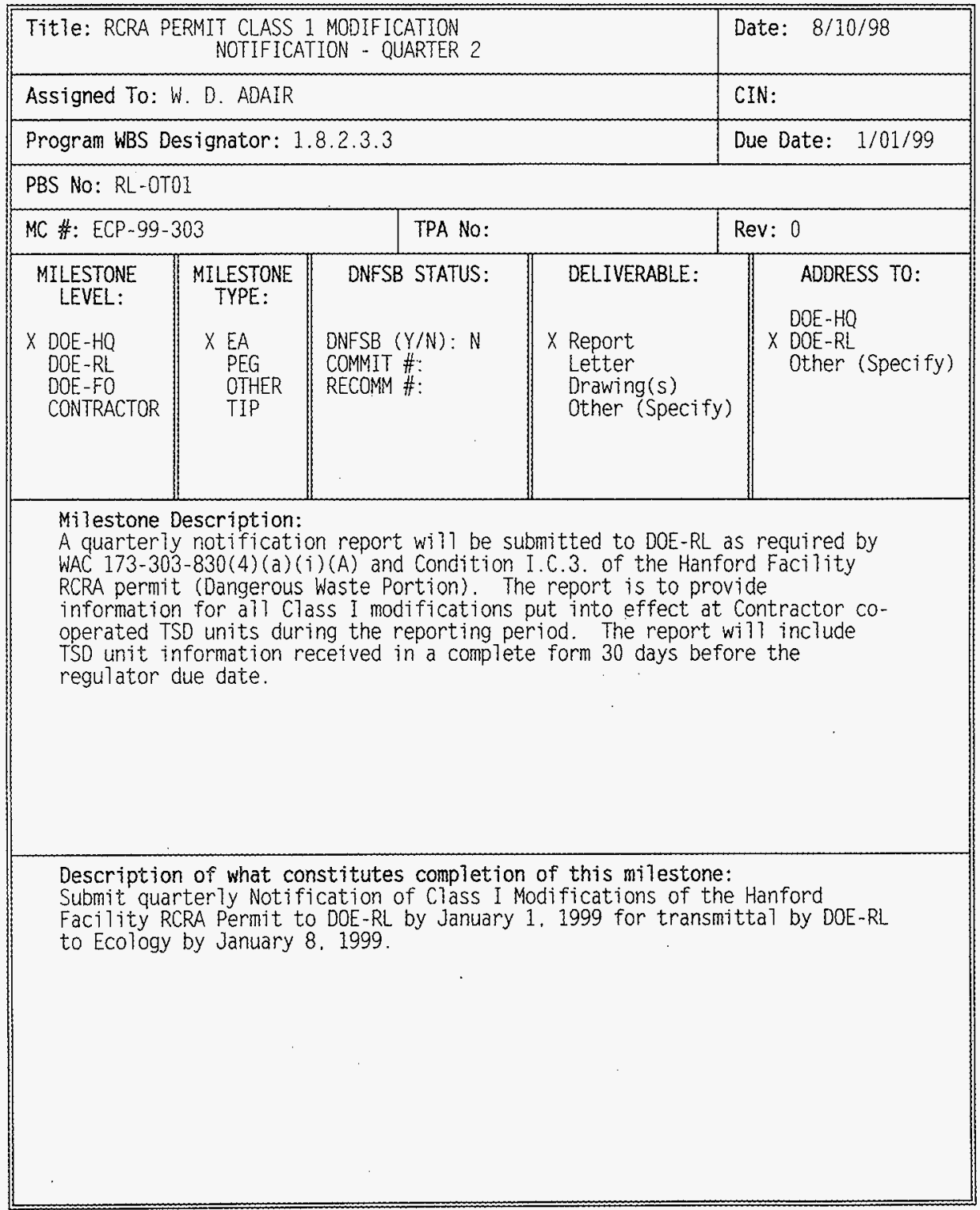


HNF-SP-1112, Rev. 4

\section{PHMC}

\section{MILESTONE DESCRIPTION SHEET}

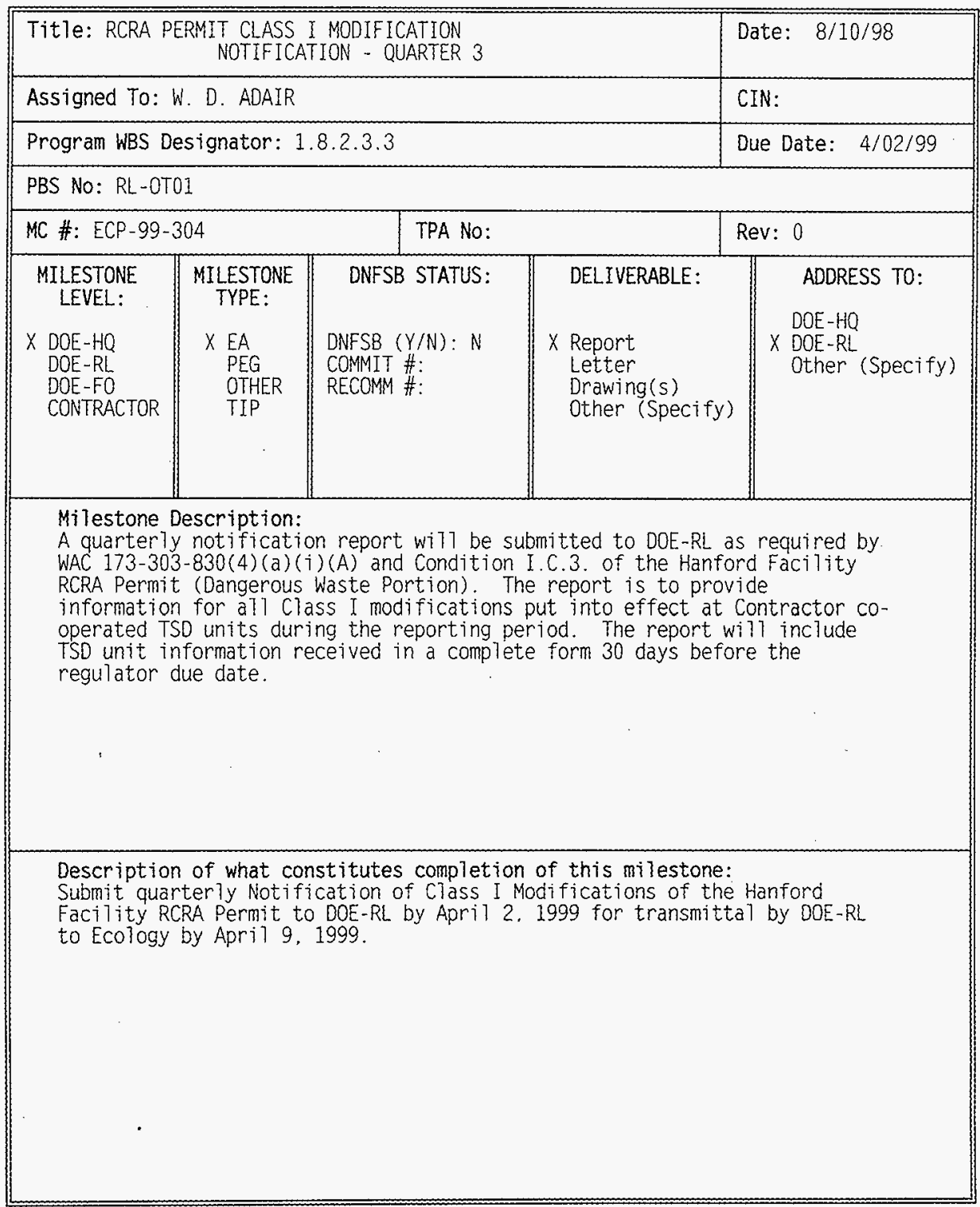


HNF-SP-1112, Rev. 4

\section{PHMC}

\section{MILESTONE DESCRIPTION SHEET}

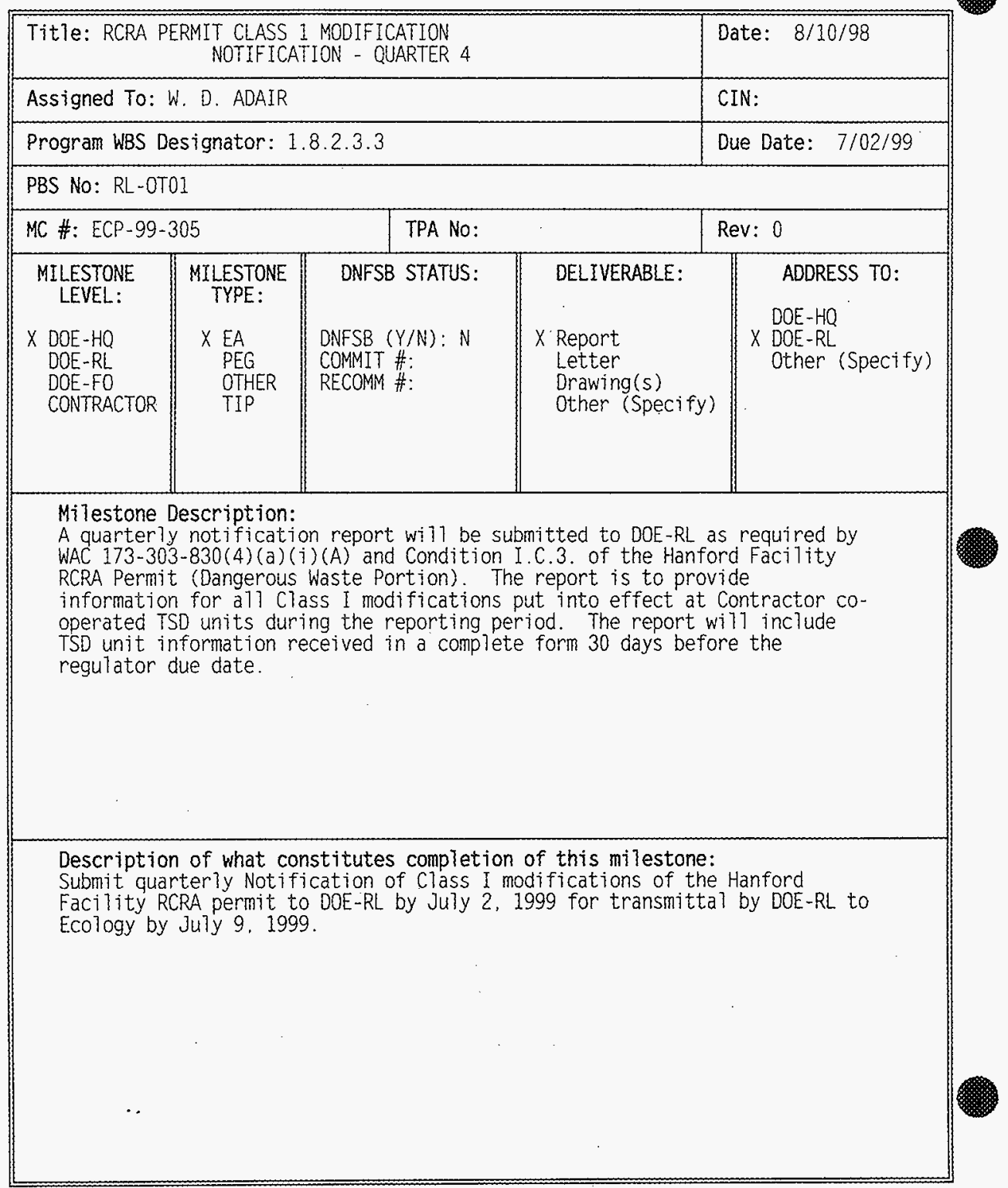




\section{PHMC}

\section{MILESTONE DESCRIPTION SHEET}

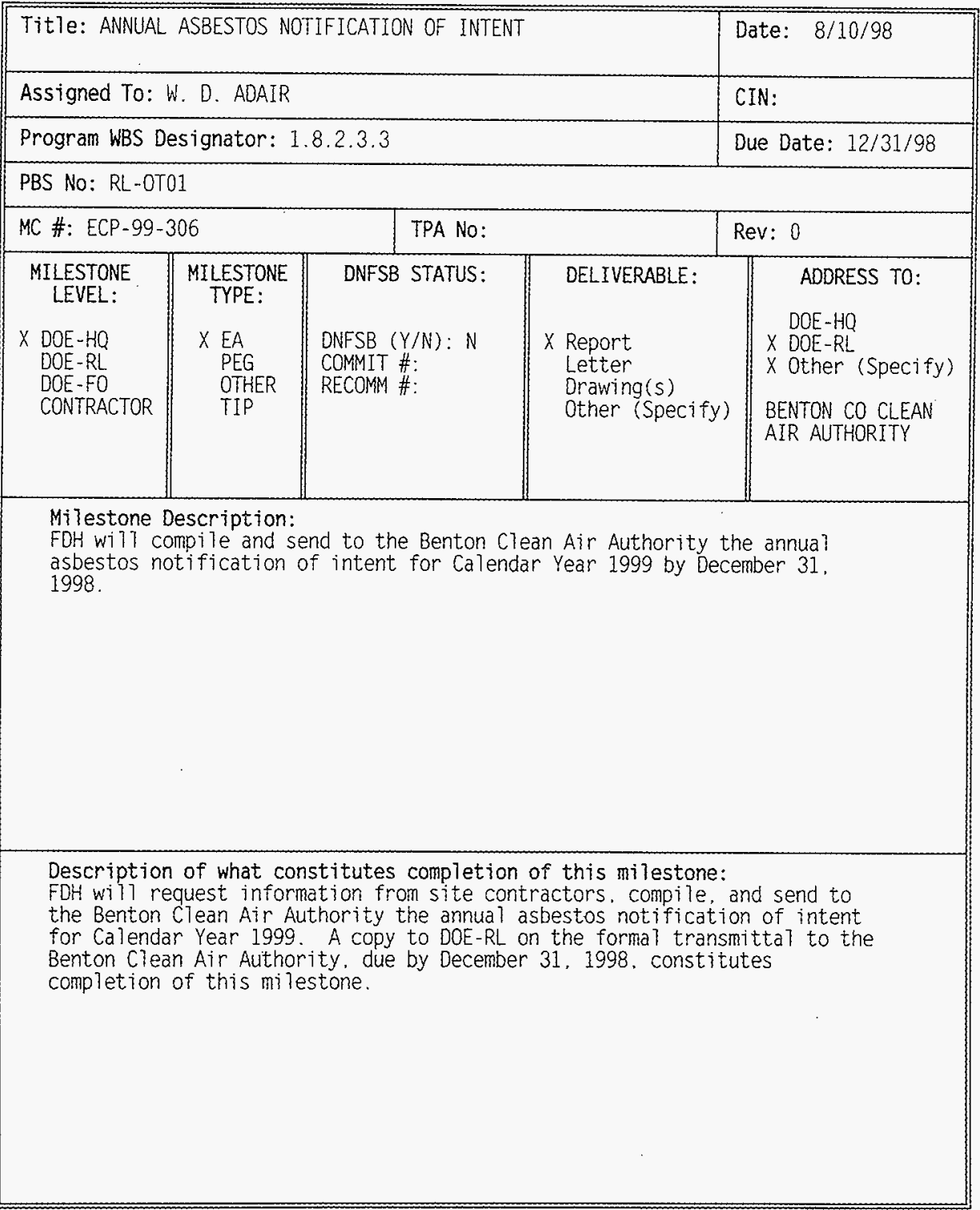




\section{PHMC}

MILESTONE DESCRIPTION SHEET

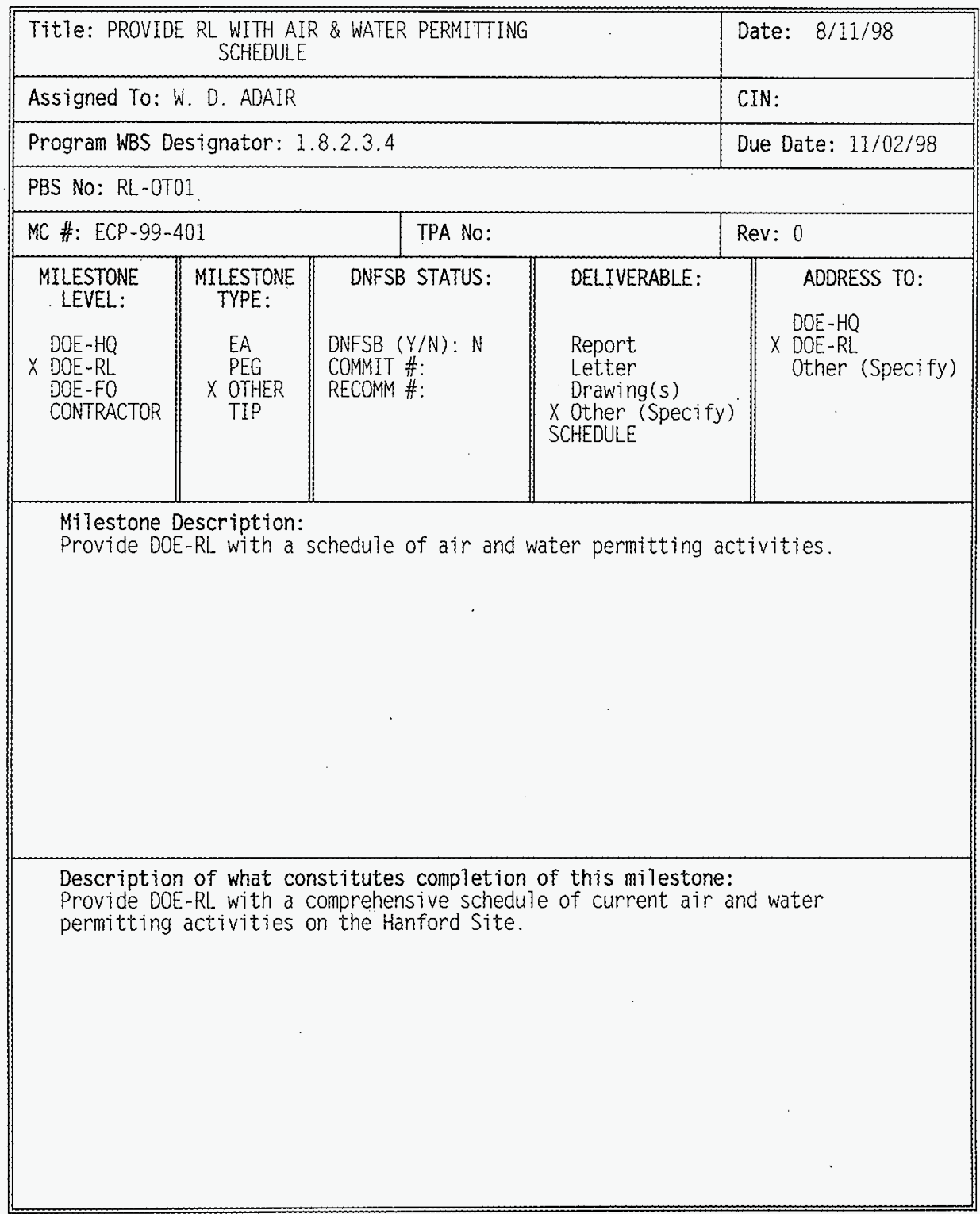


HNF-SP-1112, Rev. 4

\section{PHMC}

\section{MILESTONE DESCRIPTION SHEET}

\begin{tabular}{|c|c|c|c|c|}
\hline \multicolumn{4}{|c|}{$\begin{array}{l}\text { Title: PROVIDE RL WITH AIR AND WATER PERMITTING } \\
\text { SCHEDULE }\end{array}$} & Date: $8 / 11 / 98$ \\
\hline \multicolumn{4}{|c|}{ Assigned To: W. D. ADAIR } & CIN: \\
\hline \multicolumn{4}{|c|}{ Program WBS Designator: 1.8.2.3.4 } & Due Date: 1/04/99. \\
\hline \multicolumn{5}{|c|}{ PBS No: RL-0T01 } \\
\hline \multicolumn{2}{|c|}{ MC \#: ECP-99-402 } & \multicolumn{2}{|l|}{ TPA No: } & Rev: 0 \\
\hline $\begin{array}{l}\text { MILESTONE } \\
\text { LEVEL: } \\
\\
\text { OOE-HO } \\
\times \text { OOE-RL } \\
\text { DOE-FO } \\
\text { CONTRACTOR }\end{array}$ & $\begin{array}{l}\text { MILESTONE } \\
\text { TYPE: } \\
\\
\text { EA } \\
\text { PEG } \\
\times \text { OTHER } \\
\text { TIP }\end{array}$ & $\begin{array}{l}\text { DNFSB STATUS: } \\
\text { DNFSB (Y/N): N } \\
\text { COMMIT \#: } \\
\text { RECOMM \#: }\end{array}$ & $\begin{array}{l}\text { DELIVERABLE: } \\
\\
\text { Report } \\
\text { Letter } \\
\text { Drawing(s) } \\
\text { X Other (Specify) } \\
\text { SCHEDULE }\end{array}$ & $\begin{array}{l}\text { ADDRESS TO: } \\
\text { DOE-HQ } \\
\times \text { DOE-RL } \\
\text { Other (Specify) }\end{array}$ \\
\hline \multicolumn{5}{|c|}{$\begin{array}{l}\text { Milestone Description: } \\
\text { Provide DOE-RL with a schedule of air and water permitting activities. }\end{array}$} \\
\hline \multicolumn{5}{|c|}{$\begin{array}{l}\text { Description of what constitutes completion of this milestone: } \\
\text { Provide OOE-RL with a comprehensive schedule of current air and water } \\
\text { permitting activities on the Hanford Site. }\end{array}$} \\
\hline
\end{tabular}


HNF-SP-1112, Rev. 4

\section{PHMC}

\section{MILESTONE DESCRIPTION SHEET}

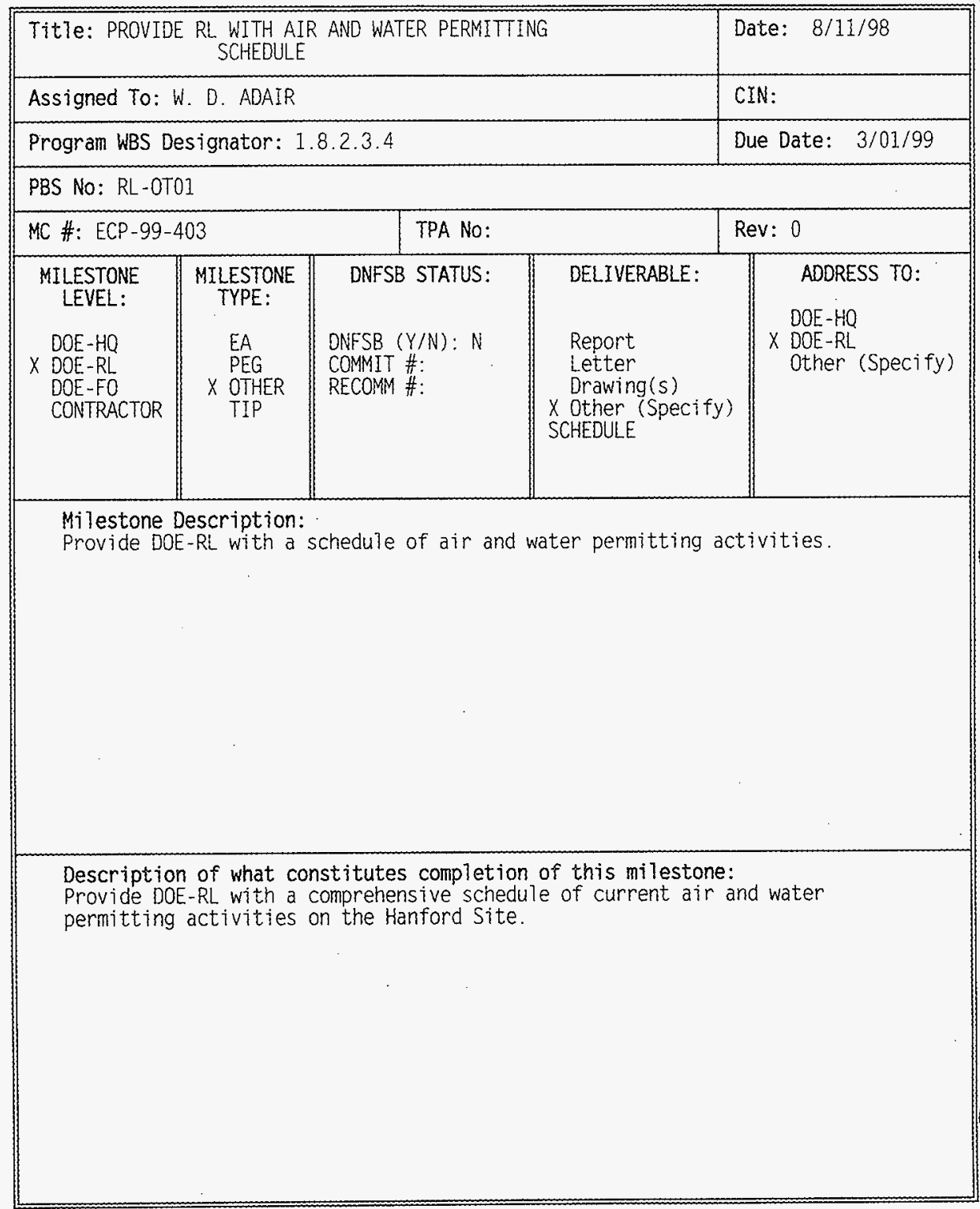




\section{PHMC \\ MILESTONE DESCRIPTION SHEET}

\begin{tabular}{|c|c|c|c|c|}
\hline \multicolumn{4}{|c|}{$\begin{array}{l}\text { TitTe: PROVIDE RL WITH AIR AND WATER PERMITTING } \\
\text { SCHEDULE. }\end{array}$} & Date: $8 / 11 / 99$ \\
\hline \multicolumn{4}{|c|}{ Assigned To: $W . D$. ADAIR } & CIN: \\
\hline \multicolumn{4}{|c|}{ Program WBS Designator: 1.8 .2 .3 .4} & Due Date: $5 / 03 / 99$ \\
\hline \multicolumn{5}{|c|}{ PBS No: RL-OT01 } \\
\hline \multicolumn{2}{|c|}{ MC \#: ECP-99-404 } & TPA No: & & Rev: 0 \\
\hline $\begin{array}{l}\text { MILESTONE } \\
\text { LEVEL: } \\
\text { DOE-HQ } \\
\text { X OOE-RL } \\
\text { DOE-FO } \\
\text { CONTRACTOR }\end{array}$ & $\begin{array}{l}\text { MILESTONE } \\
\text { TYPE: } \\
\text { EA } \\
\text { PEG } \\
\times \text { OTHER } \\
\text { TIP }\end{array}$ & $\begin{array}{l}\text { DNFSB STATUS: } \\
\text { ONFSB }(Y / N): N \\
\text { COMMIT \#: } \\
\text { RECOMM \#: }\end{array}$ & $\begin{array}{l}\text { DELIVERABLE: } \\
\text { Report } \\
\text { Letter } \\
\text { Drawing(s) } \\
\text { X Other (Specify) } \\
\text { SCHEDULE }\end{array}$ & $\begin{array}{l}\text { ADDRESS TO: } \\
\text { OOE-HO } \\
\times \text { OOE-RL } \\
\text { Other (Specify) }\end{array}$ \\
\hline \multicolumn{5}{|c|}{$\begin{array}{l}\text { Milestone Description: } \\
\text { Provide DOE-RL with a schedule of air and water permitting activities. }\end{array}$} \\
\hline \multicolumn{5}{|c|}{$\begin{array}{l}\text { Description of what constitutes completion of this milestone: } \\
\text { Provide DOE-RL with a comprehensive schedule of current air and water } \\
\text { permitting activities on the Hanford Site. }\end{array}$} \\
\hline
\end{tabular}




\begin{tabular}{|c|c|c|c|c|}
\hline \multicolumn{4}{|c|}{$\begin{array}{l}\text { Title: PROVIDE RL WITH AIR AND WATER PERMITTING } \\
\text { SCHEDULE }\end{array}$} & Date: $8 / 11 / 98$ \\
\hline \multicolumn{4}{|c|}{ Assigned To: W. D. ADAIR } & CIN: \\
\hline \multicolumn{4}{|c|}{ Program WBS Designator: $1 \cdot 8 \cdot 2 \cdot 3.4$} & Due Date: 7/01/99 \\
\hline \multicolumn{5}{|c|}{ PBS No: RL-0T01 } \\
\hline \multicolumn{2}{|c|}{ MC \#: ECP-99-405 } & TPA No: & & Rev: 0 \\
\hline $\begin{array}{l}\text { MILESTONE } \\
\text { LEVEL: } \\
\\
\text { DOE-HO } \\
\times \text { DOE-RL } \\
\text { DOE-FO } \\
\text { CONTRACTOR }\end{array}$ & $\begin{array}{l}\text { MILESTONE } \\
\text { TYPE: } \\
\\
\text { EA } \\
\text { PEG } \\
\times \text { OTHER } \\
\text { TIP }\end{array}$ & $\begin{array}{l}\text { DNFSB STATUS: } \\
\text { ONFSB (Y/N): N } \\
\text { COMMIT \#: } \\
\text { RECOMM \#: }\end{array}$ & $\begin{array}{l}\text { DELIVERABLE: } \\
\text { Report } \\
\text { Letter } \\
\text { Drawing(s) } \\
\text { X Other (Specify) } \\
\text { SCHEDULE }\end{array}$ & $\begin{array}{l}\text { ADDRESS TO: } \\
\text { DOE-HQ } \\
\times \text { DOE-RL } \\
\text { Other (Specify) }\end{array}$ \\
\hline
\end{tabular}

Description of what constitutes completion of this milestone:

Provide DOE-RL with a comprehensive schedule of current air and water permitting activities on the Hanford Site. 


\section{PHMC}

\section{MILESTONE DESCRIPTION SHEET}

\begin{tabular}{|c|c|c|c|c|}
\hline \multicolumn{4}{|c|}{$\begin{array}{c}\text { Title: PROVIDE RL WITH AIR AND WATER PERMITTING } \\
\text { SCHEOULE }\end{array}$} & Date: $8 / 11 / 99$ \\
\hline \multicolumn{4}{|c|}{ Assigned To: W. D. ADAIR } & CIN: \\
\hline \multicolumn{4}{|c|}{ Program WBS Designator: $1.8 \cdot 2 \cdot 3.4$} & Due Date: 9/01/99 \\
\hline \multicolumn{5}{|c|}{ PBS No: RL-0T01 } \\
\hline \multicolumn{2}{|c|}{ MC \#: ECP-99-406 } & \multicolumn{2}{|l|}{ TPA No: } & Rev: 0 \\
\hline $\begin{array}{l}\text { MILESTONE } \\
\text { LEVEL: }\end{array}$ & $\begin{array}{l}\text { MILESTONE } \\
\text { TYPE: }\end{array}$ & DNFSB STATUS: & DELIVERABLE: & \multirow{2}{*}{$\begin{array}{l}\text { ADDRESS TO: } \\
\text { DOE-HQ } \\
\times \text { DOE-RL } \\
\text { Other (Specify) }\end{array}$} \\
\hline $\begin{array}{l}\text { DOE-HO } \\
\times \text { DOE-RL } \\
\text { DOE-FO } \\
\text { CONTRACTOR }\end{array}$ & $\begin{array}{l}\text { EA } \\
\text { PEG } \\
\times \text { OTHER } \\
\text { TIP }\end{array}$ & $\begin{array}{l}\text { DNFSB }(Y / N): N \\
\text { COMMIT \#: } \\
\text { RECOMM \#: }\end{array}$ & $\begin{array}{l}\text { Report } \\
\text { Letter } \\
\text { Drawing (s) } \\
\text { Other (Specify) }\end{array}$ & \\
\hline
\end{tabular}

Milestone Description:

Provide DOE-RL with a schedule of air and water permitting activities.

Description of what constitutes completion of this milestone:

Provide DOE-RL with a comprehensive schedule of current air and water permitting activities on the Hanford Site. 


\section{HNF-SP-1112, Rev. 4 \\ PHMC \\ MILESTONE DESCRIPTION SHEET}

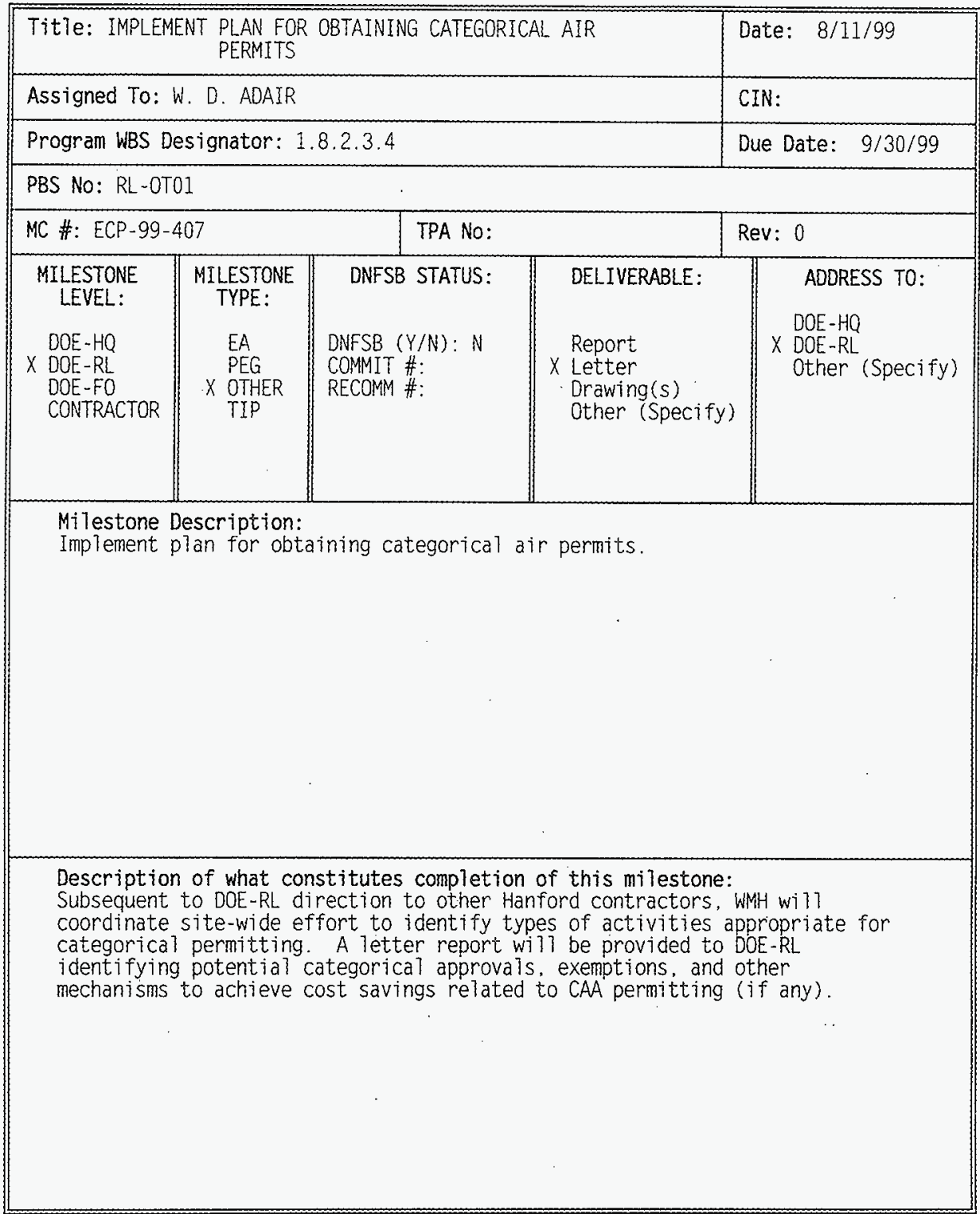


PHMC

\section{MILESTONE DESCRIPTION SHEET}

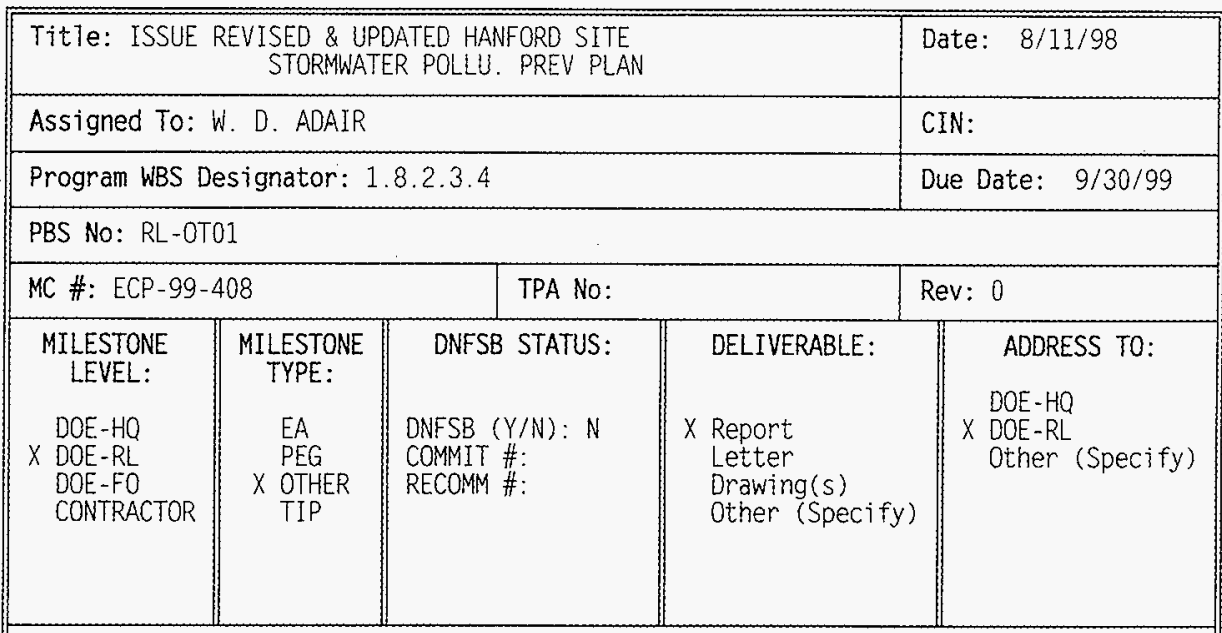

Milestone Description:

Provide DOE-RL with a revised, updated version of the Hanford Site Stormwater Pollution Prevention Plan.

Description of what constitutes completion of this milestone:

An updated, revised version of the Hanford Site Stormwater Pollution

Prevention Plan. 


\section{PHMC}

MILESTONE DESCRIPTION SHEET

\begin{tabular}{|c|c|c|c|c|}
\hline \multicolumn{4}{|c|}{ TitTe: COMPLETE SHORELINE INSPECTIONS } & Date: $8 / 11 / 98$ \\
\hline \multicolumn{4}{|c|}{ Assigned To: W. D. ADAIR } & CIN: \\
\hline \multicolumn{4}{|c|}{ Program WBS Designator: 1.8.2.3.4 } & Due Date: $9 / 30 / 99$ \\
\hline \multicolumn{5}{|c|}{ PBS No: RL-0T01 } \\
\hline \multicolumn{2}{|c|}{ MC \#: ECP-99-409 } & TPA No: & & Rev: 0 \\
\hline $\begin{array}{l}\text { MILESTONE } \\
\text { LEVEL: } \\
\times \text { DOE-HO } \\
\text { OOE-RL } \\
\text { DOE-FO } \\
\text { CONTRACTOR }\end{array}$ & $\begin{array}{l}\text { MILESTONE } \\
\text { TYPE: } \\
\text { X EA } \\
\text { PEG } \\
\text { OTHER } \\
\text { TIP }\end{array}$ & $\begin{array}{l}\text { DNFSB STATUS: } \\
\text { ONFSB }(Y / N): N \\
\text { COMMIT \#: } \\
\text { RECOMM \#: }\end{array}$ & $\begin{array}{l}\text { DELIVERABLE: } \\
\text { X Report } \\
\text { Letter } \\
\text { Drawing(s) } \\
\text { Other (Specify) }\end{array}$ & $\begin{array}{l}\text { ADDRESS TO: } \\
\times \text { DOE-HQ } \\
\times \text { DOE-RL } \\
\text { Other (Specify) }\end{array}$ \\
\hline \multicolumn{5}{|c|}{$\begin{array}{l}\text { Milestone Description: } \\
\text { Complete shoreline inspections necessary to prepare and compile the annual } \\
\text { Hanford site Stormwater Comprehensive site Compliance Evaluation Report. }\end{array}$} \\
\hline \multicolumn{5}{|c|}{$\begin{array}{l}\text { Description of what constitutes completion of this milestone: } \\
\text { Shoreline inspections will be completed. }\end{array}$} \\
\hline
\end{tabular}


HNF-SP-1112, Rev. 4

\section{PHMC \\ MILESTONE DESCRIPTION SHEET}

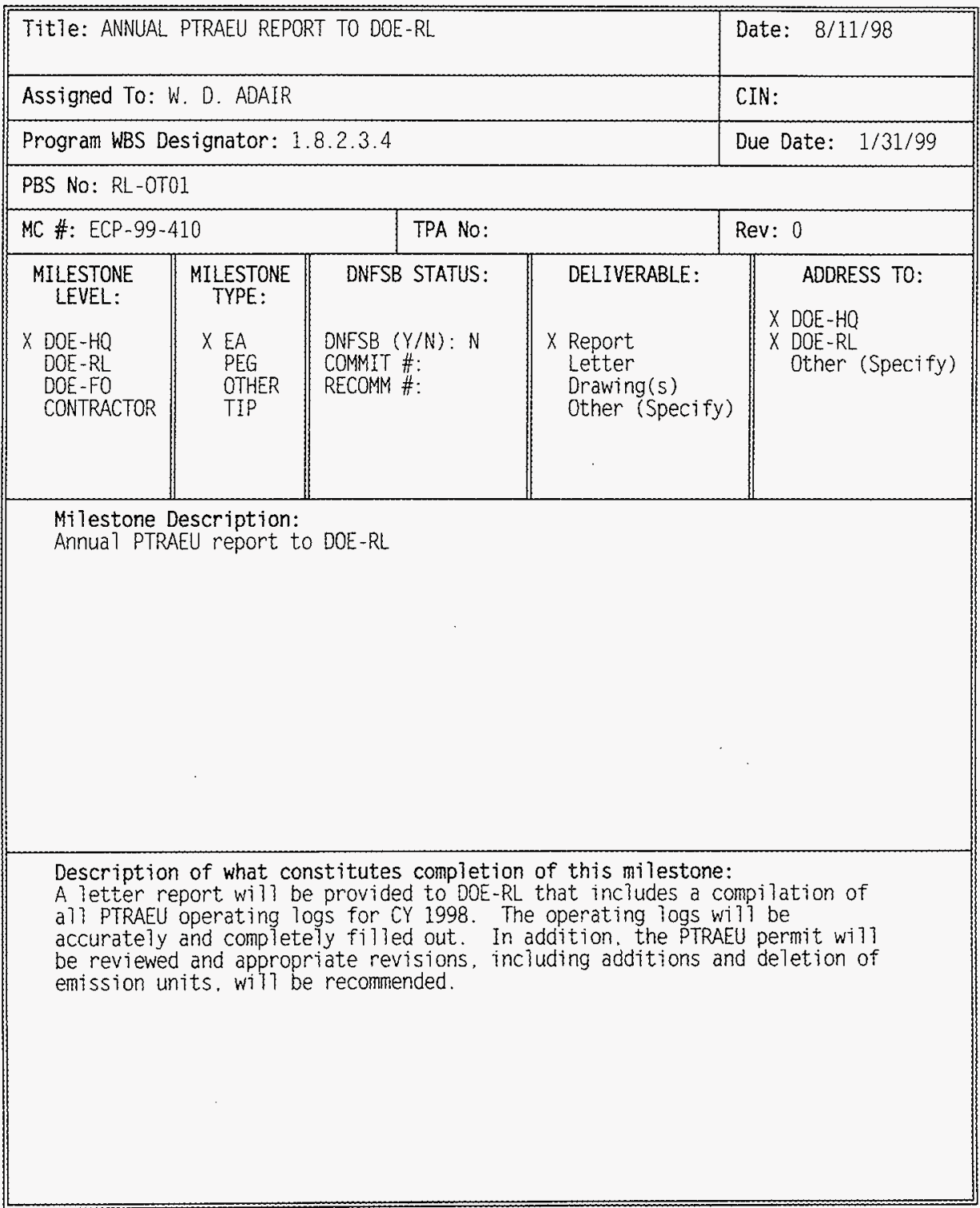




\section{PHMC}

MILESTONE DESCRIPTION SHEET

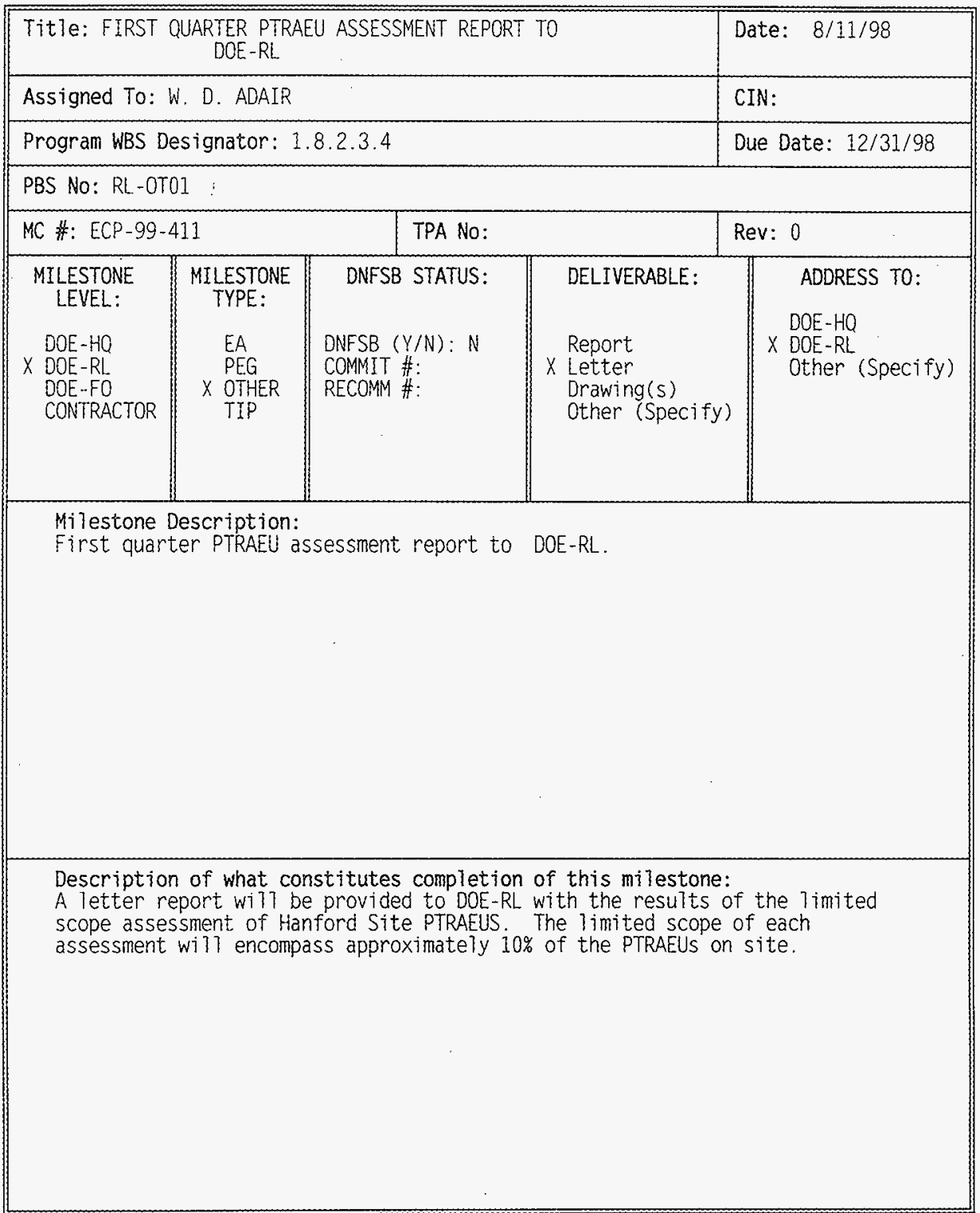


HNF-SP-1112, Rev. 4

\section{PHMC}

\section{MILESTONE DESCRIPTION SHEET}

\begin{tabular}{|c|c|c|c|c|}
\hline \multicolumn{4}{|c|}{$\begin{array}{c}\text { Titie: SECOND QUARTER PTRAEU ASSESSMENT REPORT TO } \\
\text { DOE-RL }\end{array}$} & Date: $8 / 11 / 99$ \\
\hline \multicolumn{4}{|c|}{ Assigned To: W. D. ADAIR } & CIN: \\
\hline \multicolumn{4}{|c|}{ Program WBS Designator: 1.8.2.3.4 } & Due Date: $3 / 31 / 99$ \\
\hline \multicolumn{5}{|c|}{ PBS No: RL-OT01 } \\
\hline \multicolumn{2}{|c|}{$M C \#: E C P-99-412$} & \multicolumn{2}{|l|}{ TPA No: } & Rev: 0 \\
\hline $\begin{array}{l}\text { MILESTONE } \\
\text { LEVEL: }\end{array}$ & $\begin{array}{l}\text { MILESTONE } \\
\text { TYPE: }\end{array}$ & DNFSB STATUS: & OELIVERABLE: & ADDRESS TO: \\
\hline $\begin{array}{l}\text { DOE-HO } \\
\times \text { DOE-RL } \\
\text { DOE-FO } \\
\text { CONTRACTOR }\end{array}$ & $\begin{array}{l}\text { EA } \\
\text { PEG } \\
\times \text { OTHER } \\
\text { TIP }\end{array}$ & $\begin{array}{l}\text { DNFSB }(Y / N): N \\
\text { COMMIT \#: } \\
\text { RECOMM \#: }\end{array}$ & $\begin{array}{l}\text { Report } \\
x \text { Letter } \\
\text { Drawing(s) } \\
\text { Other (Specify) }\end{array}$ & $\begin{array}{l}\times \text { DOE-RL } \\
\text { Other (Specify) }\end{array}$ \\
\hline
\end{tabular}

Milestone Description:

Second quarter PTRAEU assessment report to DOE-RL.

Description of what constitutes completion of this milestone:

A letter report will be provided to DOE-RL with the results of a limited scope assessment of Hanford Site PTRAEUS. The Timited scope of each

assessment wi11 encompass approximately $10 \%$ of the PTRAEUS on site. 
HNF-SP-1112, Rev. 4

\section{PHMC}

MILESTONE DESCRIPTION SHEET

\begin{tabular}{|c|c|c|c|c|}
\hline \multicolumn{4}{|c|}{$\begin{array}{l}\text { Title: THIRD QUARTER PTRAEU ASSESSMENT REPORT TO } \\
\text { DOE-RL }\end{array}$} & Date: $8 / 11 / 98$ \\
\hline \multicolumn{4}{|c|}{ Assigned To: W. D. ADAIR } & CIN: \\
\hline \multicolumn{4}{|c|}{ Program WBS Designator: $1.8 \cdot 2.3 .4$} & Due Date: $6 / 30 / 99$ \\
\hline \multicolumn{5}{|c|}{ PBS NO: RL-OT01 } \\
\hline \multicolumn{2}{|c|}{ MC \#: ECP-99-413 } & \multicolumn{2}{|l|}{ TPA No: } & Rev: 0 \\
\hline $\begin{array}{l}\text { MILESTONE } \\
\text { LEVEL: } \\
\text { DOE-HQ } \\
\text { X DOE-RL } \\
\text { DOE-FO } \\
\text { CONTRACTOR }\end{array}$ & $\begin{array}{l}\text { MILESTONE } \\
\text { TYPE: } \\
\\
\text { EA } \\
\text { PEG } \\
\times \text { OTHER } \\
\text { TIP }\end{array}$ & $\begin{array}{l}\text { DNFSB STATUS: } \\
\text { DNFSB }(Y / N): N \\
\text { COMMIT \#: } \\
\text { RECOMM \#: }\end{array}$ & $\begin{array}{l}\text { DELIVERABLE: } \\
\\
\text { Report } \\
\text { x Letter } \\
\text { Drawing (s) } \\
\text { Other (Specify) }\end{array}$ & $\begin{array}{l}\text { ADDRESS TO: } \\
\text { DOE-HQ } \\
\times \text { DOE-RL } \\
\text { Other (Specify) }\end{array}$ \\
\hline
\end{tabular}

Description of what constitutes completion of this milestone:

A letter report will be provided to DOE-RL with the results of a limited scope assessment of Hanford Site PTRAEUS. The 7 imited scope of each assessment will encompass approximately $10 \%$ of the PTRAEUS on site. 


\section{PHMC}

MILESTONE DESCRIPTION SHEET

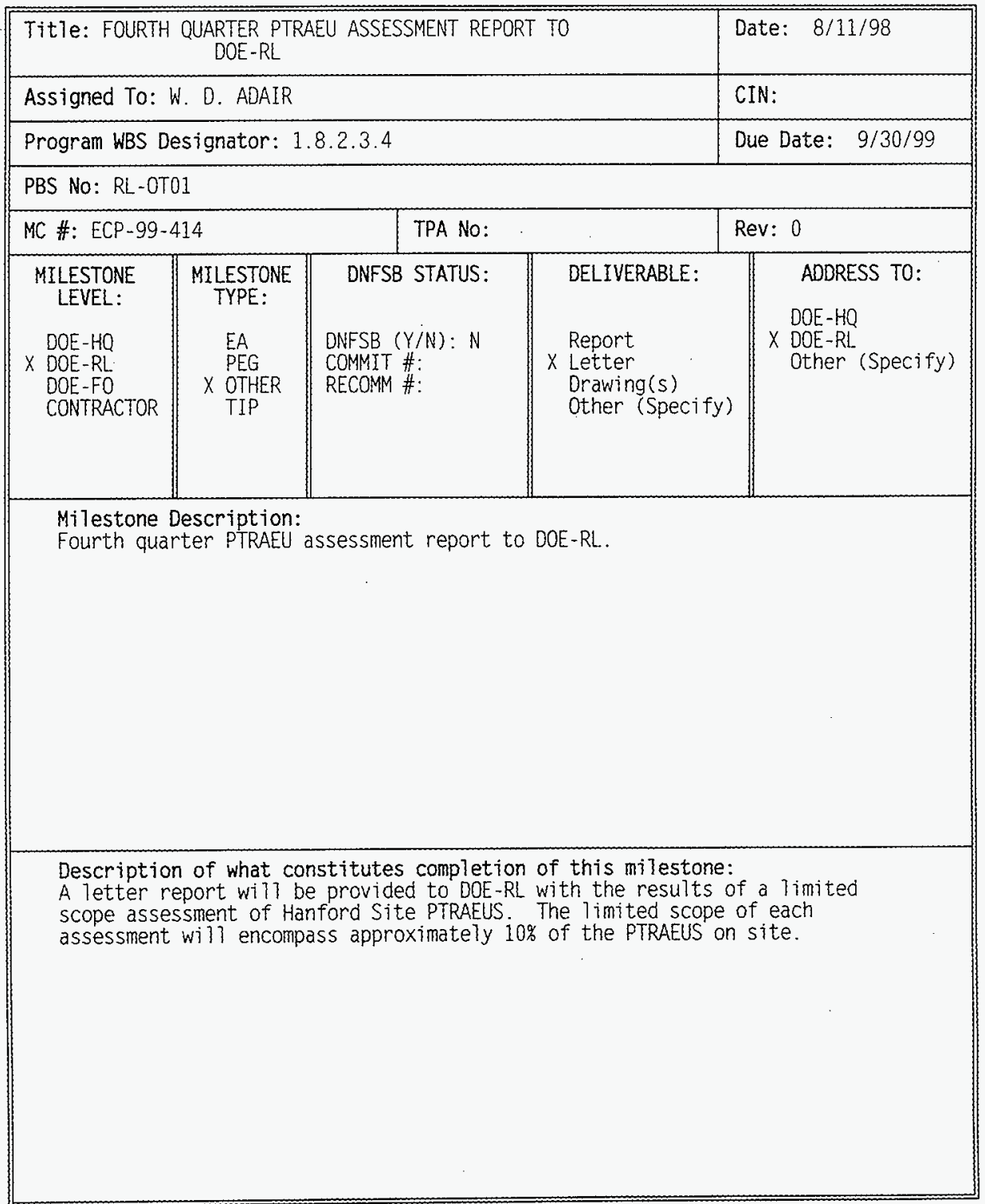




\section{PHMC \\ MILESTONE DESCRIPTION SHEET}

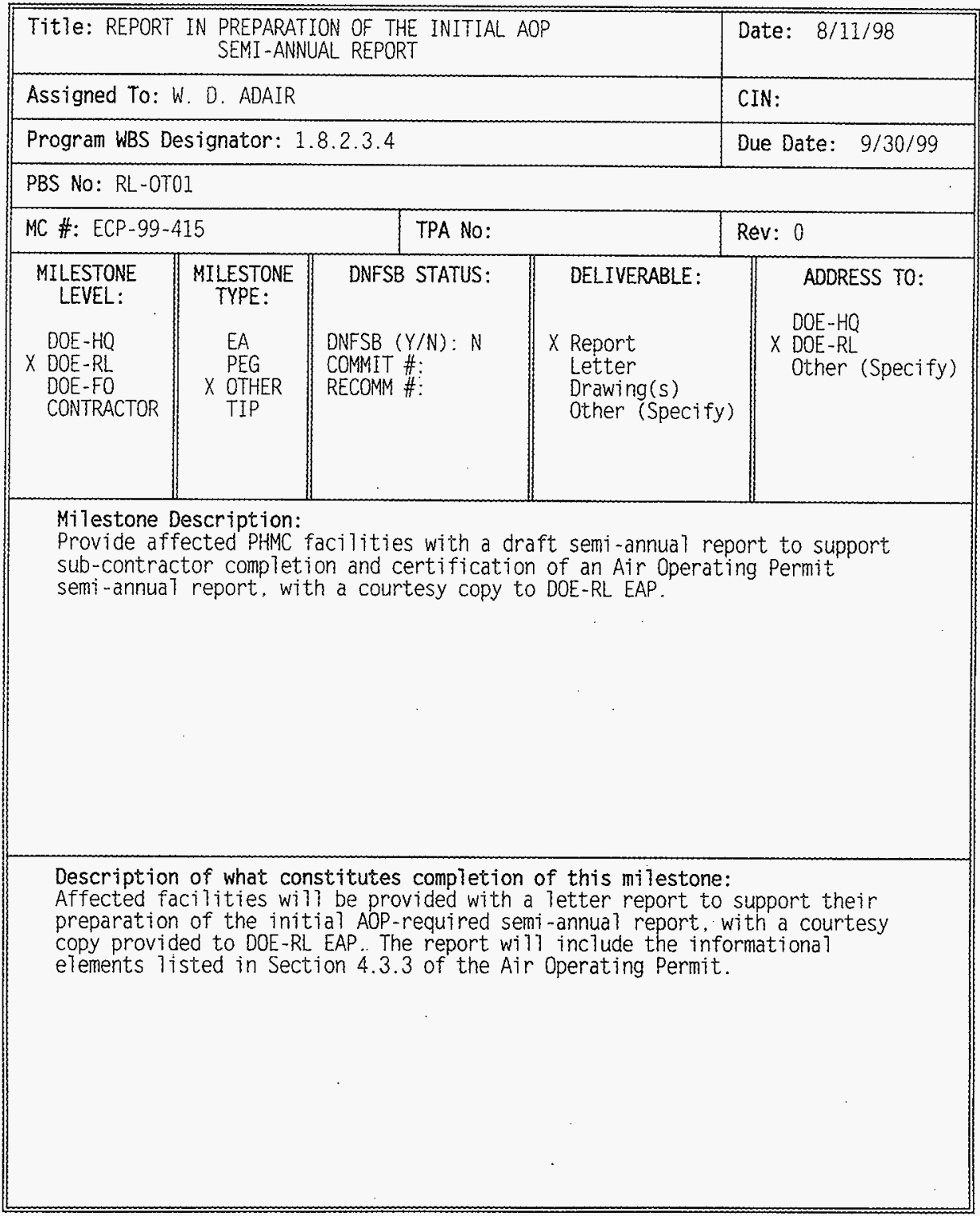


HNF-SP-1112, Rev. 4

\section{PHMC \\ MILESTONE DESCRIPTION SHEET}

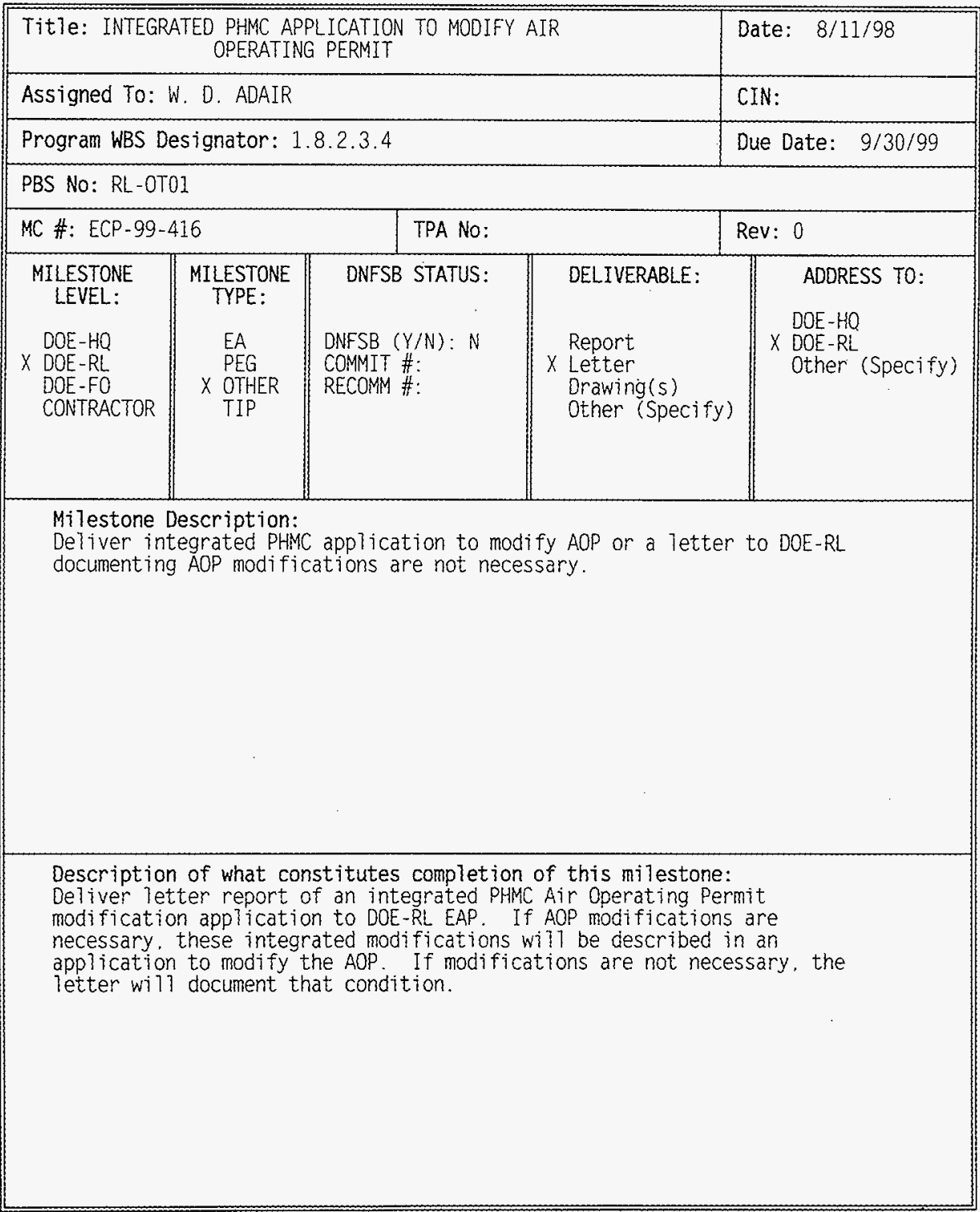




\section{PHMC}

\section{MILESTONE DESCRIPTION SHEET}

\begin{tabular}{|c|c|c|c|c|}
\hline \multicolumn{4}{|c|}{$\begin{array}{l}\text { Title: INTEGRATED AIR OPERATING PERMIT SEMIANNUAL } \\
\text { REPORT }\end{array}$} & Date: $8 / 11 / 98$ \\
\hline \multicolumn{4}{|c|}{ Assigned To: W. D. ADAIR } & CIN: \\
\hline \multicolumn{4}{|c|}{ Program WBS Designator: $1.8 \cdot 2.3 .4$} & Due Date: $9 / 30 / 99$ \\
\hline \multicolumn{5}{|c|}{ PBS NO: RL-OTO1 } \\
\hline \multicolumn{2}{|c|}{ MC \#: ECP-99-417 } & TPA No: & & Rev: 0 \\
\hline $\begin{array}{l}\text { MILESTONE } \\
\text { LEVEL: } \\
\times \text { DOE-HQ } \\
\text { DOE-RL } \\
\text { DOE-FO } \\
\text { CONTRACTOR }\end{array}$ & $\begin{array}{l}\text { MILESTONE } \\
\text { TYPE: } \\
\times \text { EA } \\
\text { PEG } \\
\text { OTHER } \\
\text { TIP }\end{array}$ & $\begin{array}{l}\text { DNFSB STATUS: } \\
\text { DNFSB }(Y / N): N \\
\text { COMMIT \#: } \\
\text { RECOMM \#: }\end{array}$ & $\begin{array}{l}\text { DELIVERABLE: } \\
\times \text { Report } \\
\text { Letter } \\
\text { Drawing(s) } \\
\text { Other (Specify) }\end{array}$ & $\begin{array}{l}\text { ADDRESS TO: } \\
\text { DOE-HO } \\
\times \text { DOE-RL } \\
\text { Other (Specify) }\end{array}$ \\
\hline \multicolumn{5}{|c|}{$\begin{array}{l}\text { Milestone Description: } \\
\text { Provide integrated PHMC semiannual report required by AOP to DOE-RL EAP. }\end{array}$} \\
\hline \multicolumn{5}{|c|}{$\begin{array}{l}\text { Description of what constitutes completion of this milestone: } \\
\text { Provide DOE-RL with a semiannual report. certified by FDH as true, } \\
\text { accurate, and complete, to support transmittal to the regulatory agencies, } \\
\text { to satisfy the terms and conditions of the AOP. }\end{array}$} \\
\hline
\end{tabular}


HNF-SP-1112, Rev. 4

PHMC

MILESTONE DESCRIPTION SHEET

\begin{tabular}{|c|c|c|c|c|}
\hline \multicolumn{4}{|c|}{ Title: FINAL AOP IMPLEMENTATION PLAN } & Date: $8 / 11 / 98$ \\
\hline \multicolumn{4}{|c|}{ Assigned To: W. D. ADAIR } & CIN: \\
\hline \multicolumn{4}{|c|}{ Program WBS Designator: 1.8 .2 .3 .4} & Due Date: $9 / 30 / 99$ \\
\hline \multicolumn{5}{|c|}{ PBS No: RL-OT01 } \\
\hline \multicolumn{2}{|c|}{ MC \#: ECP-99-418 } & TPA No: & & Rev: 0 \\
\hline $\begin{array}{l}\text { MILESTONE } \\
\text { LEVEL: }\end{array}$ & $\begin{array}{l}\text { MILESTONE } \\
\text { TYPE: }\end{array}$ & DNFSB STATUS: & DELIVERABLE: & \multirow{2}{*}{$\begin{array}{l}\text { ADDRESS TO: } \\
\text { DOE-HQ } \\
\times \text { DOE-RL } \\
\text { Other (Specify) }\end{array}$} \\
\hline $\begin{array}{l}\text { DOE-HQ } \\
\times \text { DOE-RL } \\
\text { DOE-FO } \\
\text { CONTRACTOR }\end{array}$ & $\begin{array}{l}\text { EA } \\
\text { PEG } \\
\times \quad \text { OTHER } \\
\text { TIP }\end{array}$ & $\begin{array}{l}\text { DNFSB }(Y / N): N \\
\text { COMMIT \#: } \\
\text { RECOMM \#: }\end{array}$ & $\begin{array}{l}X \text { Report } \\
\text { Letter } \\
\text { Drawing(s) } \\
\text { Other (Specify) }\end{array}$ & \\
\hline
\end{tabular}

Milestone Description:

Deliver final implementation pian to DOE-RL.

Description of what constitutes completion of this milestone:

Following the format and content agreed to for the draft implementation plan, submit the final version of the AOP Implementation Plan to DOE-RL. Comments from Hanford Site contractors and DOE-RL on the draft plan will be incorporated into the final plan. where appropriate. 


\section{PHMC}

\section{MILESTONE DESCRIPTION SMEET}

\begin{tabular}{|c|c|c|c|c|}
\hline \multicolumn{4}{|c|}{ TitTe: DELIVER FINAL RISK MANAGEMENT PLAN TO DOE-RL } & Date: $8 / 11 / 98$ \\
\hline \multicolumn{4}{|c|}{ Assigned To: $W$. D. ADAIR } & CIN: \\
\hline \multicolumn{4}{|c|}{ Program WBS Designator: $1 \cdot 8 \cdot 2 \cdot 3 \cdot 4$} & Due Date: $6 / 07 / 99$ \\
\hline \multicolumn{5}{|c|}{ PBS No: RL-0T01 } \\
\hline \multicolumn{2}{|c|}{ MC \#: ECP-99-419 } & TPA No: & & Rev: 0 \\
\hline $\begin{array}{l}\text { MILESTONE } \\
\text { LEVEL: } \\
\times \text { DOE-HQ } \\
\text { DOE-RL } \\
\text { DOE-FO } \\
\text { CONTRACTOR }\end{array}$ & $\begin{array}{l}\text { MILESTONE } \\
\text { TYPE: } \\
\times \text { EA } \\
\text { PEG } \\
\text { OTHER } \\
\text { TIP }\end{array}$ & $\begin{array}{l}\text { DNFSB STATUS: } \\
\text { DNFSB (Y/N): N } \\
\text { COMMIT \#: } \\
\text { RECOMM \#: }\end{array}$ & $\begin{array}{l}\text { DELIVERABLE: } \\
\text { X Report } \\
\text { Letter } \\
\text { Orawing(s) } \\
\text { Other (Specify) }\end{array}$ & $\begin{array}{l}\text { ADDRESS TO: } \\
\text { DOE-HQ } \\
\times \text { DOE-RL } \\
\text { Other (Specify) }\end{array}$ \\
\hline
\end{tabular}

Description of what constitutes completion of this milestone:

Provide DOE-RL with a statement certifying a risk management $p l a n$ has been determined not to apply to the Hanford Site or a Risk Management Plan and registration form. 


\section{PHMC}

\section{MILESTONE DESCRIPTION SHEET}

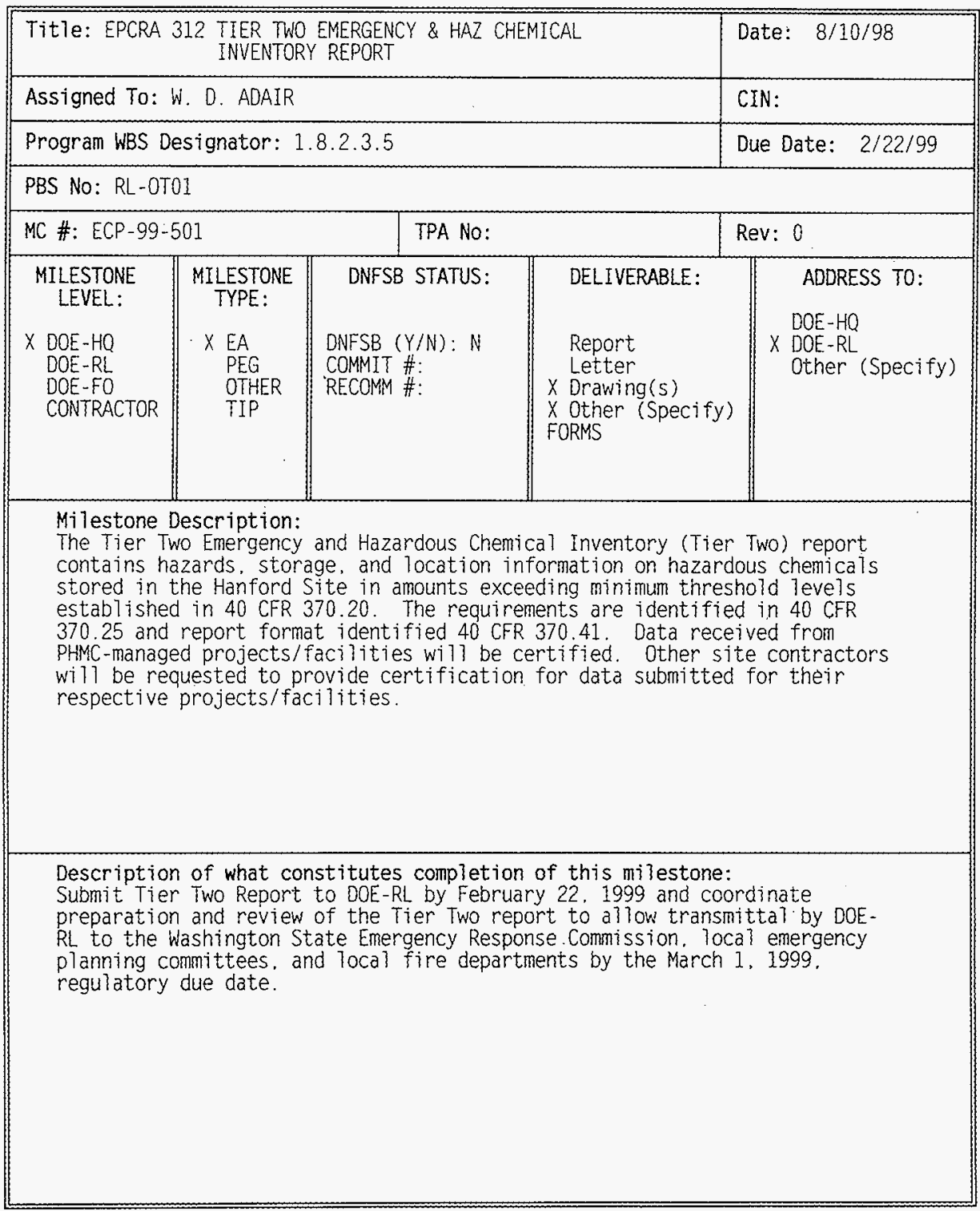


HNF-SP-1112, Rev. 4

PHMC

MILESTONE DESCRIPTION SHEET

\begin{tabular}{|c|c|c|c|c|}
\hline \multicolumn{4}{|c|}{$\begin{array}{c}\text { Title: } 1998 \text { HANFORD SITE ANNUAL DANGEROUS WASTE } \\
\text { REPORT }\end{array}$} & Date: $8 / 10 / 98$ \\
\hline \multicolumn{4}{|c|}{ Assigned To: $W .0$. ADAIR } & CIN: \\
\hline \multicolumn{4}{|c|}{ Program WBS Designator: 1.8 .2 .3 .5} & Due Date: 2/22/99 \\
\hline \multicolumn{5}{|c|}{ PBS No: RL-0TO1 } \\
\hline \multicolumn{2}{|c|}{ MC \#: ECP-99-503 } & TPA No: & & Rev: 0 \\
\hline $\begin{array}{l}\text { MILESTONE } \\
\text { LEVEL: } \\
\text { X DOE-HQ } \\
\text { DOE-RL } \\
\text { DOE-FO } \\
\text { CONTRACTOR }\end{array}$ & $\begin{array}{l}\text { MILESTONE } \\
\text { TYPE: } \\
\text { X EA } \\
\text { PEG } \\
\text { OTHER } \\
\text { TIP }\end{array}$ & $\begin{array}{l}\text { DNFSB STATUS: } \\
\text { DNFSB }(Y / N): N \\
\text { COMMIT \#: } \\
\text { RECOMM \#: }\end{array}$ & $\begin{array}{l}\text { DELIVERABLE: } \\
\times \text { Report } \\
\text { Letter } \\
\text { Drawing (s) } \\
\times \text { Other (Specify) } \\
\text { DATA SHEETS }\end{array}$ & $\begin{array}{l}\text { ADDRESS TO: } \\
\text { DOE-HQ } \\
\times \text { DOE-RL } \\
\text { Other (Specify) }\end{array}$ \\
\hline \multicolumn{5}{|c|}{$\begin{array}{l}\text { Milestone Description: } \\
\text { The Annual Dangerous Waste Report is a compilation of the DoE dangerous } \\
\text { waste generation and waste management activities on the Hanford Site for } \\
\text { the calendar year. Data received from PHMC managed projects/facilities } \\
\text { will be certified. Other site prime contractors will be requested to } \\
\text { provide certification for data that is submitted for their respective } \\
\text { projects/facilities. The report is prepared annual7y in accordance with } \\
\text { WAC 173-303-220. WAC 173-303-390, and the Hanford Facility RCRA Permit, } \\
\text { Revision 4A. Permit Condition I.E.22. (Dangerous Waste Portion). }\end{array}$} \\
\hline \multicolumn{5}{|c|}{$\begin{array}{l}\text { Description of what constitutes completion of this milestone: } \\
\text { Submit } 1998 \text { Hanford Site Annual Dangerous waste report for each EPA/State } \\
\text { I.D. number to DOE-RL by February 22. I999. For subsequent transmittal by } \\
\text { DOE-RL to Ecology and EPA by March 1. 1999. }\end{array}$} \\
\hline
\end{tabular}




\section{PHMC}

MILESTONE DESCRIPTION SHEET

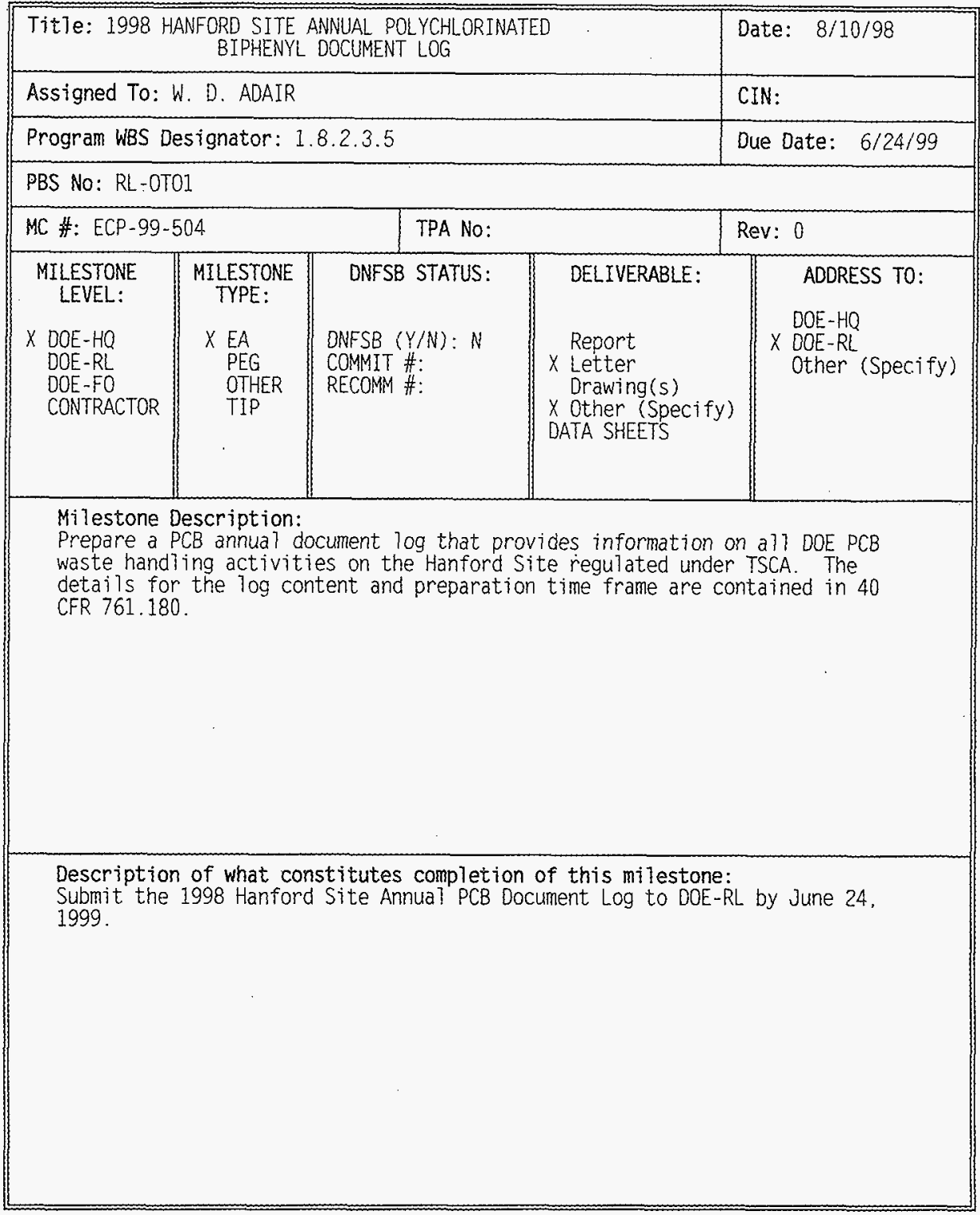




\section{HNF-SP-1112, Rev. 4 \\ PHMC \\ MILESTONE DESCRIPTION SHEET}

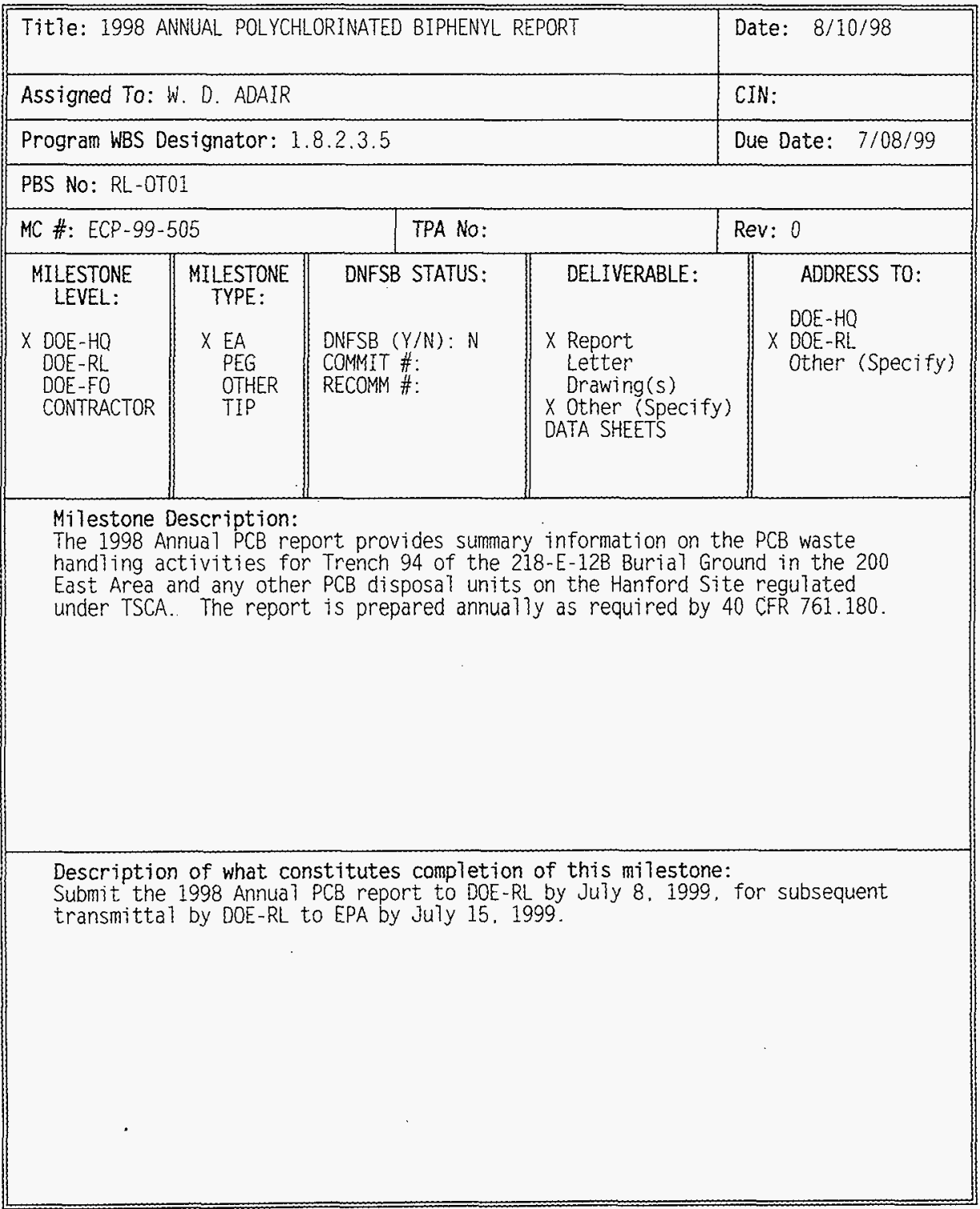




\section{PHMC}

MILESTONE DESCRIPTION SHEET

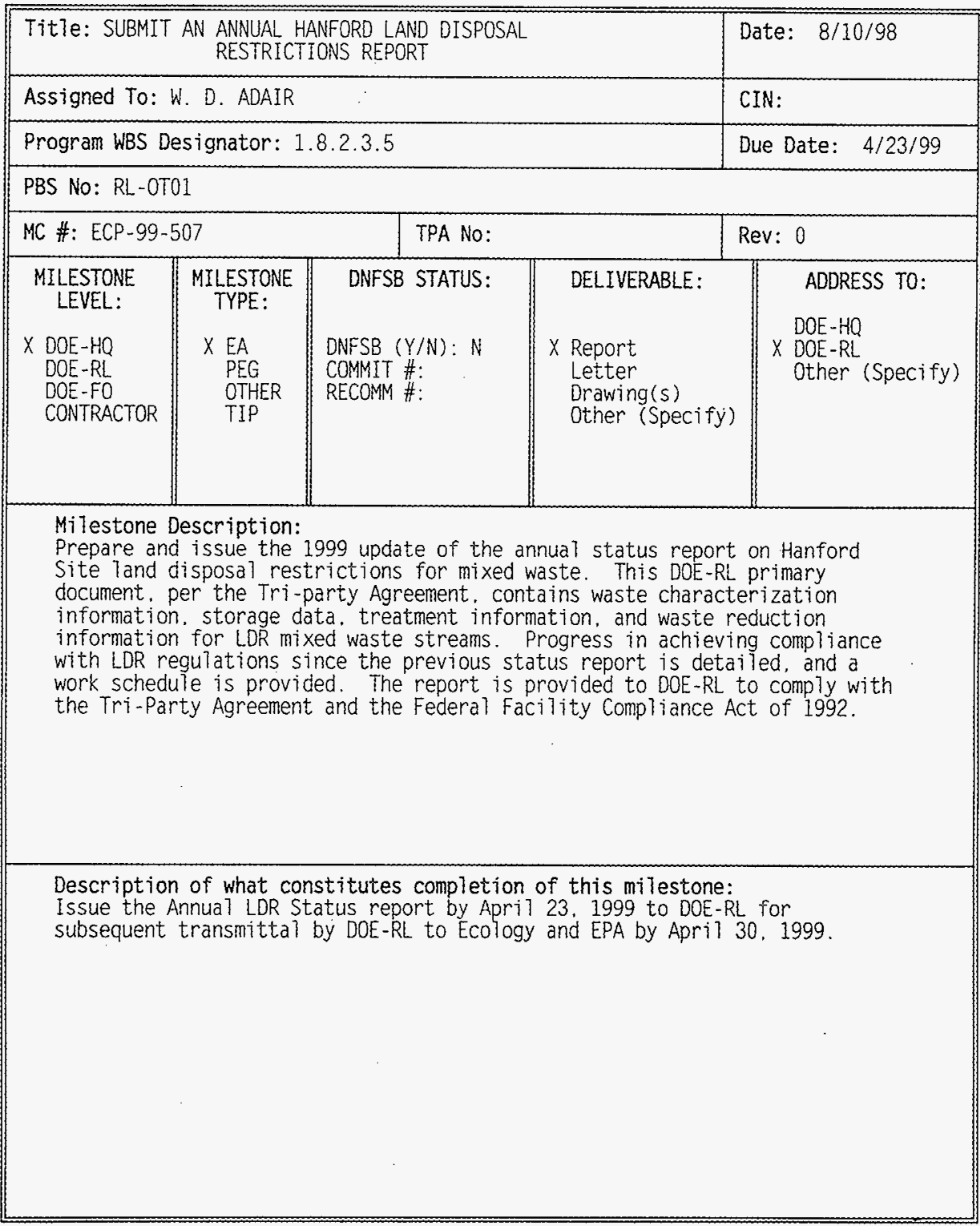


PHMC

MILESTONE DESCRIPTION SHEET

\begin{tabular}{|c|c|c|c|c|}
\hline \multicolumn{4}{|c|}{$\begin{array}{c}\text { Title: ANNUAL REPORT OF HANFORD FACILITY RCRA } \\
\text { PERMIT NONCOMPL IANCES }\end{array}$} & Date: $8 / 04 / 98$ \\
\hline \multicolumn{4}{|c|}{ Assigned To: W. D. ADAIR } & CIN: \\
\hline \multicolumn{4}{|c|}{ Program WBS Designator: 1.8.2.3.7 } & Due Date: $2 / 24 / 99$ \\
\hline \multicolumn{5}{|c|}{ PBS No: RL-OT01 } \\
\hline \multicolumn{2}{|c|}{ MC \#: ECP-99-701 } & \multicolumn{2}{|l|}{ TPA NO: } & Rev: 0 \\
\hline $\begin{array}{l}\text { MILESTONE } \\
\text { LEVEL: }\end{array}$ & $\begin{array}{l}\text { MILESTONE } \\
\text { TYPE: }\end{array}$ & DNFSB STATUS: & DELIVERABLE: & ADDRESS TO: \\
\hline $\begin{array}{l}X \text { DOE-HQ } \\
\text { DOE-RL } \\
\text { DOE-FO } \\
\text { CONTRACTOR }\end{array}$ & $\begin{array}{l}X \text { EA } \\
\text { PEG } \\
\text { OTHER } \\
\text { TIP }\end{array}$ & $\begin{array}{l}\text { DNFSB }(Y / N): N \\
\text { COMMIT \#: } \\
\text { RECOMM \#: }\end{array}$ & $\begin{array}{l}X \text { Report } \\
X \text { Letter } \\
\text { Orawing (s) } \\
\text { Other (Specify) }\end{array}$ & $\begin{array}{l}X \text { DOE-RL } \\
\text { Other (Specify) }\end{array}$ \\
\hline
\end{tabular}

Milestone Description:

Submit to DOE-RL a report that addresses the requirement in Condition I.E.19 of the Hanford Facility RCRA Permit at the time the Annual Dangerous Waste Report is submitted. This report will identify all instances of noncompliances for standard and general permit conditions at Contractor cooperated TSD units not otherwise required to be reported elsewhere in the Hanford Facility RCRA Permit (Dangerous Waste Portion). The report of noncompliances, which is for calendar year 1998. will contain information on other Contractor co-operated TSD unit noncompliances received 55 days before the regulator due date of March 1. 1999.

Description of what constitutes completion of this milestone: FOH will submit Annual Report of Hanford Faci1ity RCRA Permit Noncomp 3 iances to DOE-RL by February 24, 1999. for subsequent transmittal by DOE-RL to Ecology by March 1, 1999. 


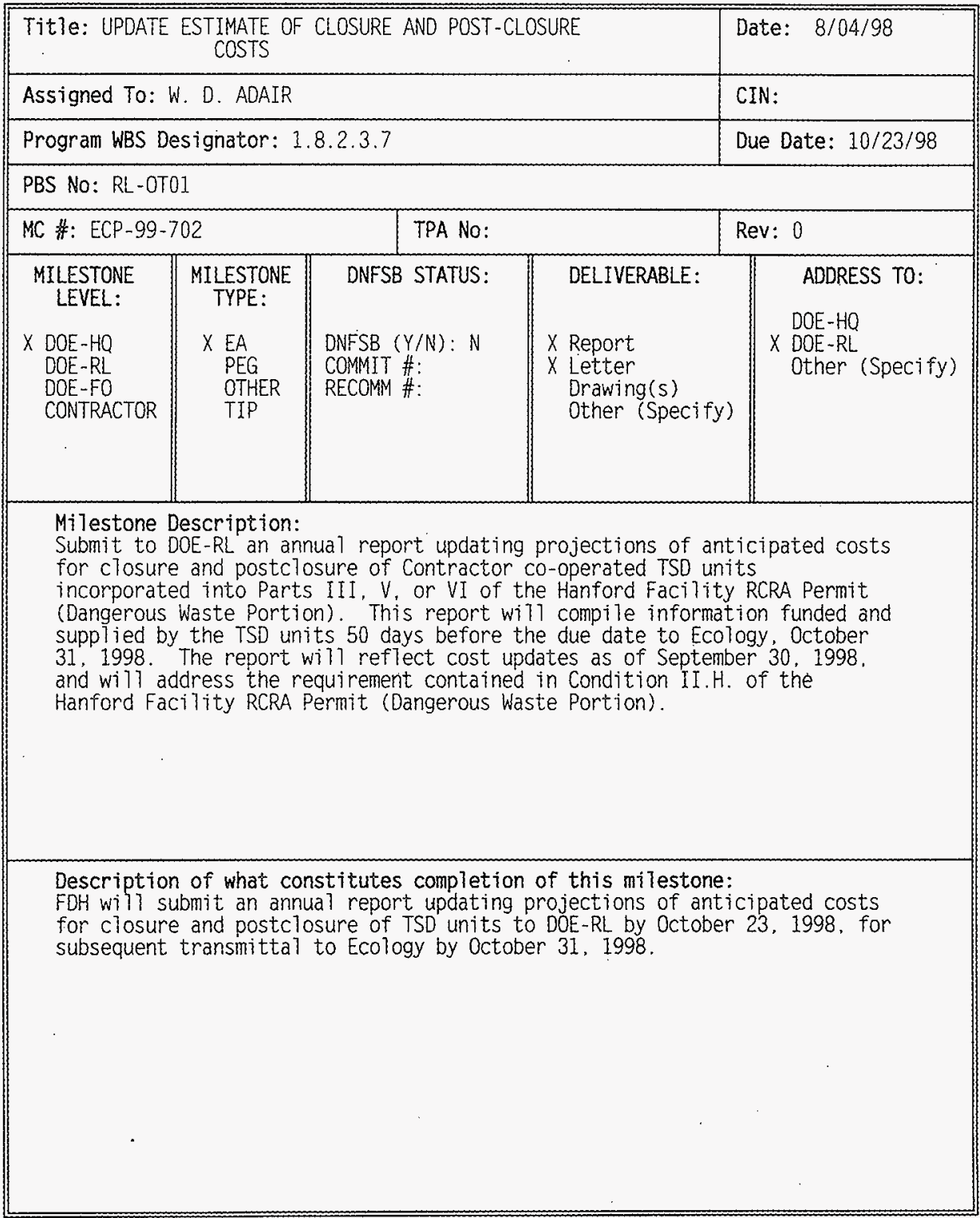




\section{PHMC \\ MILESTONE DESCRIPTION SHEET}

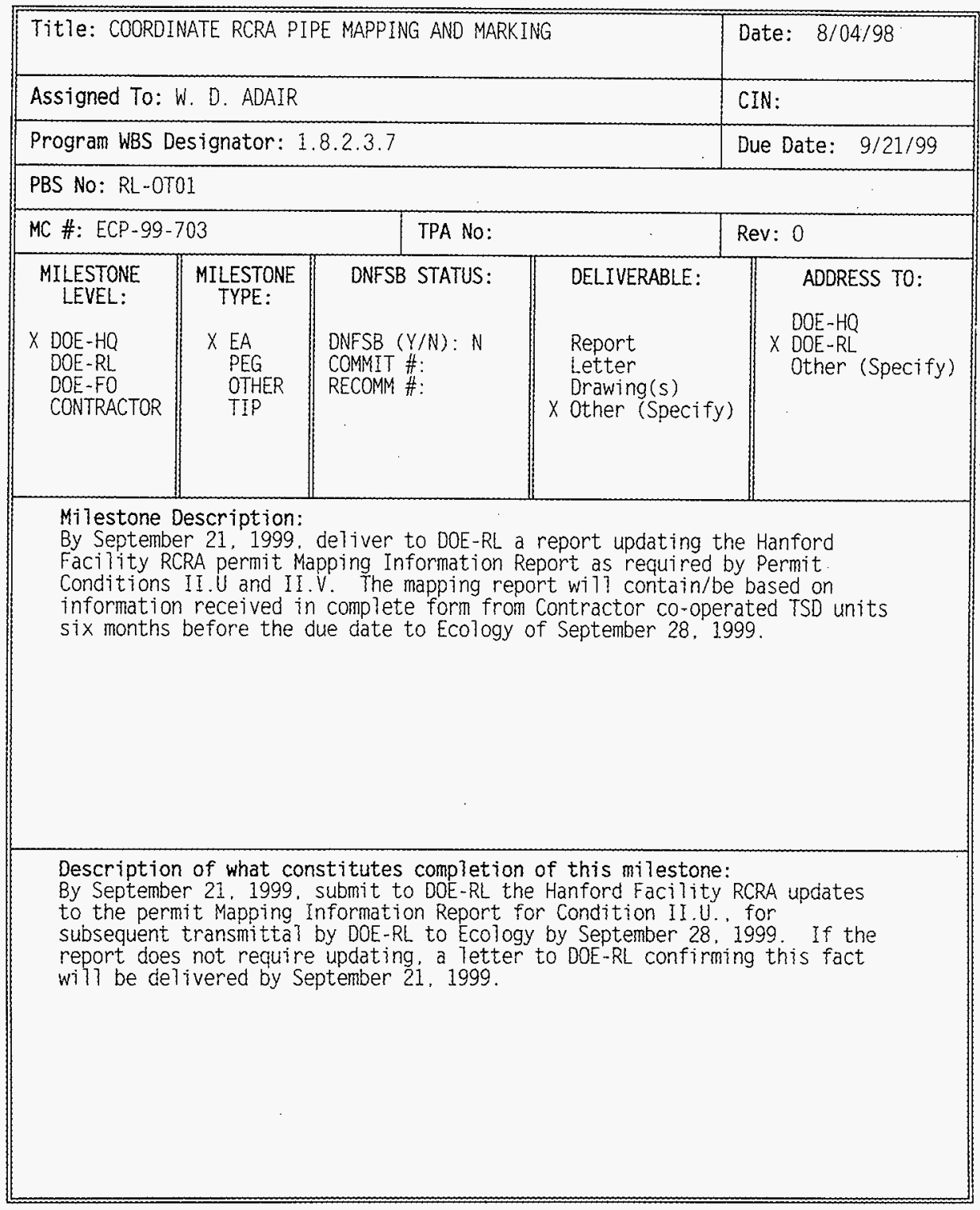




\section{PHMC \\ MILESTONE DESCRIPTION SHEET}

\begin{tabular}{|c|c|c|c|c|}
\hline \multicolumn{4}{|c|}{ Title: TRANSMIT EIS/ODIS DATA TO INEEL } & Date: $8 / 03 / 98$ \\
\hline \multicolumn{4}{|c|}{ Assigned To: W. D. ADAIR } & CIN: \\
\hline \multicolumn{4}{|c|}{ Program WBS Designator: 1.8.2.3.8 } & Due Date: 4/01/99 \\
\hline \multicolumn{5}{|c|}{ PBS No: RL-OTOI } \\
\hline \multicolumn{2}{|c|}{ MC \#: ECP-99-801 } & TPA No: & & Rev: 0 \\
\hline $\begin{array}{l}\text { MILESTONE } \\
\text { LEVEL: } \\
\text { X DOE-HO } \\
\text { DOE-RL } \\
\text { DOE-FO } \\
\text { CONTRACTOR }\end{array}$ & $\begin{array}{l}\text { MILESTONE } \\
\text { TYPE: } \\
\times \text { EA } \\
\text { PEG } \\
\text { OTHER } \\
\text { TIP }\end{array}$ & $\begin{array}{l}\text { DNFSB STATUS: } \\
\text { DNFSB (Y/N): N } \\
\text { COMMIT \#: } \\
\text { RECOMM \#: }\end{array}$ & $\begin{array}{l}\text { OELIVERABLE: } \\
\text { Report } \\
\text { Letter } \\
\text { Drawing(s) } \\
\text { X Other (Specify) } \\
\text { ELECTRONIC DATA }\end{array}$ & $\begin{array}{l}\text { ADORESS TO: } \\
\text { DOE-HO } \\
\text { DOE-RL } \\
\times \text { Other (Specify) } \\
\text { IDAHO NATIONAL } \\
\text { ENG LAB (DOE) }\end{array}$ \\
\hline \multicolumn{5}{|c|}{$\begin{array}{l}\text { Milestone Description: } \\
\text { DOE requires its sites to annually compile and send radionuclide release } \\
\text { data, for both liquid and airborne discharges, to the Idaho National } \\
\text { Environmenta } 1 \text { Engineering Laboratory (INEEL) in Idaho Falls, Idaho. in } \\
\text { accordance with DoE Order } 5400 \text {. I. This data-collecting program is called } \\
\text { the Effluent information System - Onsite Discharge Information System } \\
\text { (EIS/ODIS). NOTE: Early transmission to DOE-RL is not required. The } \\
\text { electronic format is not reviewed by DOE-RL. }\end{array}$} \\
\hline \multicolumn{5}{|c|}{$\begin{array}{l}\text { Description of what constitutes completion of this milestone: } \\
\text { Electronic transmittal to INEEL by midnight 04/01/99, and formal } \\
\text { transmittal of a completion letter to DOE-RL constitutes completion. }\end{array}$} \\
\hline
\end{tabular}




\section{HNF-SP-1112, Rev. 4 \\ PHMC \\ MILESTONE DESCRIPTION SHEET}

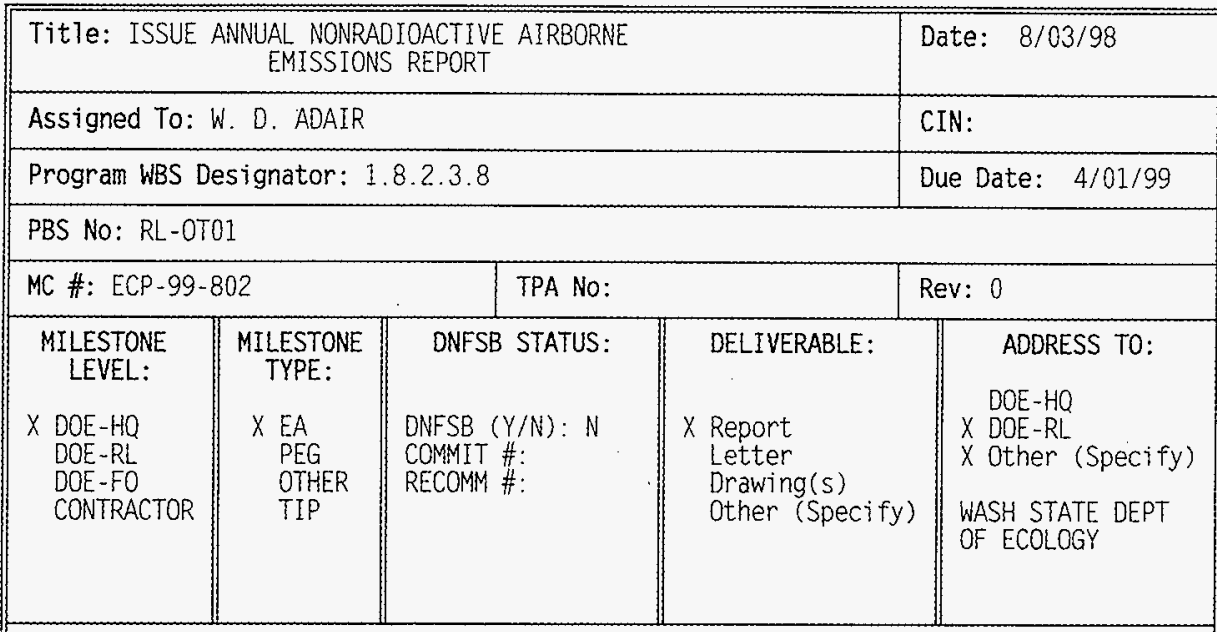

Milestone Description:

The nonradioactive airborne emissions report contains information on BHI and FDH managed operations having the potential to emit combustion products from fossil fuels and inorganic compounds from waste storage tanks.

Preparation of this report requires that nonradioactive airborne emissions data will be recorded on emissions inventory forms. Completed inventory forms will be provided to DOE-RL for transmittal to the Washington State Department of Ecology.

Description of what constitutes completion of this milestone:

Formal transmittal of the report to DOE-RL constitutes completion. DOE-RL must transmit this report to arrive at Ecology by $4 / 15 / 99$. 


\section{PHMC}

MILESTONE DESCRIPTION SHEET

\begin{tabular}{|c|c|c|c|c|}
\hline \multicolumn{4}{|c|}{$\begin{array}{c}\text { Title: ISSUE ANNUAL RADIONUCLIDE AIR EMISSIONS } \\
\text { REPORT }\end{array}$} & Date: $8 / 03 / 98$ \\
\hline \multicolumn{4}{|c|}{ Assigned To: $W$. D. ADAIR } & CIN: \\
\hline \multicolumn{4}{|c|}{ Program WBS Designator: 1.8 .2 .3 .8} & Due Date: $6 / 15 / 99$ \\
\hline \multicolumn{5}{|c|}{ PBS No: RL-0T01 } \\
\hline \multicolumn{2}{|c|}{ MC \#: ECP-99-803 } & TPA No: & & Rev: 0 \\
\hline $\begin{array}{l}\text { MILESTONE } \\
\text { LEVEL: } \\
\text { X DOE-HQ } \\
\text { DOE-RL } \\
\text { DOE-FO } \\
\text { CONTRACTOR }\end{array}$ & $\begin{array}{l}\text { MILESTONE } \\
\text { TYPE: } \\
\times \text { EA } \\
\text { PEG } \\
\quad \text { OTHER } \\
\text { TIP }\end{array}$ & $\begin{array}{l}\text { DNFSB STATUS: } \\
\text { DNFSB }(Y / N): N \\
\text { COMMIT \#: } \\
\text { RECOMM \#: }\end{array}$ & $\begin{array}{l}\text { DELIVERABLE: } \\
\times \text { Report } \\
\text { Letter } \\
\text { Orawing(s) } \\
\text { Other (Specify) }\end{array}$ & $\begin{array}{l}\text { ADDRESS TO: } \\
\text { DOE-HQ } \\
\times \text { DOE-RL } \\
\times \text { Other (Specify) } \\
\text { EPA }\end{array}$ \\
\hline \multicolumn{5}{|c|}{$\begin{array}{l}\text { Milestone Description: } \\
\text { The Radionuclide Air Emissions Report for the Hanford Site is due to the } \\
\text { EPA by } 06 / 30 \text { of each year following the year being reported in accordance } \\
\text { with } 40 \text { CFR } 61 \text {. Subpart } H \text {. The report is prepared for DOE-RL and contains } \\
\text { data on radionuclides emitted to the atmosphere from Hanford Site } \\
\text { facilities managed by PNNL, BHI, and FDH. }\end{array}$} \\
\hline \multicolumn{5}{|c|}{$\begin{array}{l}\text { Description of what constitutes completion of this milestone: } \\
\text { Formal transmitta? of the finaT report to DOE-RL constitutes completion } \\
\text { Transmittal shall occur ten working days prior to } 06 / 30 \text {, and contain ali } \\
\text { Hanford contractor signatures of certification. }\end{array}$} \\
\hline
\end{tabular}




\section{PHMC}

\section{MILESTONE DESCRIPTION SHEET}

\begin{tabular}{|c|c|c|c|c|}
\hline \multicolumn{4}{|c|}{$\begin{array}{c}\text { Title: ISSUE ANNUAL REPORT ON ENVIRONMENTAL } \\
\text { RELEASES }\end{array}$} & Date: $8 / 03 / 98$ \\
\hline \multicolumn{4}{|c|}{ Assigned To: $W$. D. ADAIR } & CIN: \\
\hline \multicolumn{4}{|c|}{ Program WBS Designator: 1.8 .2 .3 .8} & Due Date: $8 / 31 / 99$ \\
\hline \multicolumn{5}{|c|}{ PBS No: RL-OT01 } \\
\hline \multicolumn{2}{|c|}{ MC \#: ECP-99-804 } & TPA NO: & & Rev: 0 \\
\hline $\begin{array}{l}\text { MILESTONE } \\
\text { LEVEL: } \\
\times \text { DOE-HQ } \\
\text { OOE-RL } \\
\text { DOE-FO } \\
\text { CONTRACTOR }\end{array}$ & $\begin{array}{l}\text { MILESTONE } \\
\text { TYPE: } \\
\times \text { EA } \\
\text { PEG } \\
\text { OTHER } \\
\text { TIP }\end{array}$ & $\begin{array}{l}\text { DNFSB STATUS: } \\
\text { DNFSB }(Y / N): N \\
\text { COMMIT \#: } \\
\text { RECOMM \#: }\end{array}$ & $\begin{array}{l}\text { DELIVERABLE: } \\
\times \text { Report } \\
\text { Letter } \\
\text { Drawing (s) } \\
\text { Other (Specify) }\end{array}$ & $\begin{array}{l}\text { ADDRESS TO: } \\
\text { DOE-HQ } \\
\times \text { DOE-RL } \\
\text { Other (Specify) }\end{array}$ \\
\hline
\end{tabular}

Milestone Description:

This annual report presents data for radioactive and nonradioactive substances released into the environment during the previous calendar year from the Hanford Site facilities managed by FDH and BHI. Both summary and detailed presentations of this data are given, and some comparisons to data from previous years are made. Although not an enforceable agreement milestone, it does document compliance with DOE Order 5400.1

Description of what constitutes completion of this milestone:

This report is not transmitted by DOE-RL to other agencies. Forma? transmittal of the report to DOE-RL by August 31. 1999, with contractor signatures of approval constitutes completion. 


\section{PHMC \\ MILSTONE DESCRIPTION SHEET}

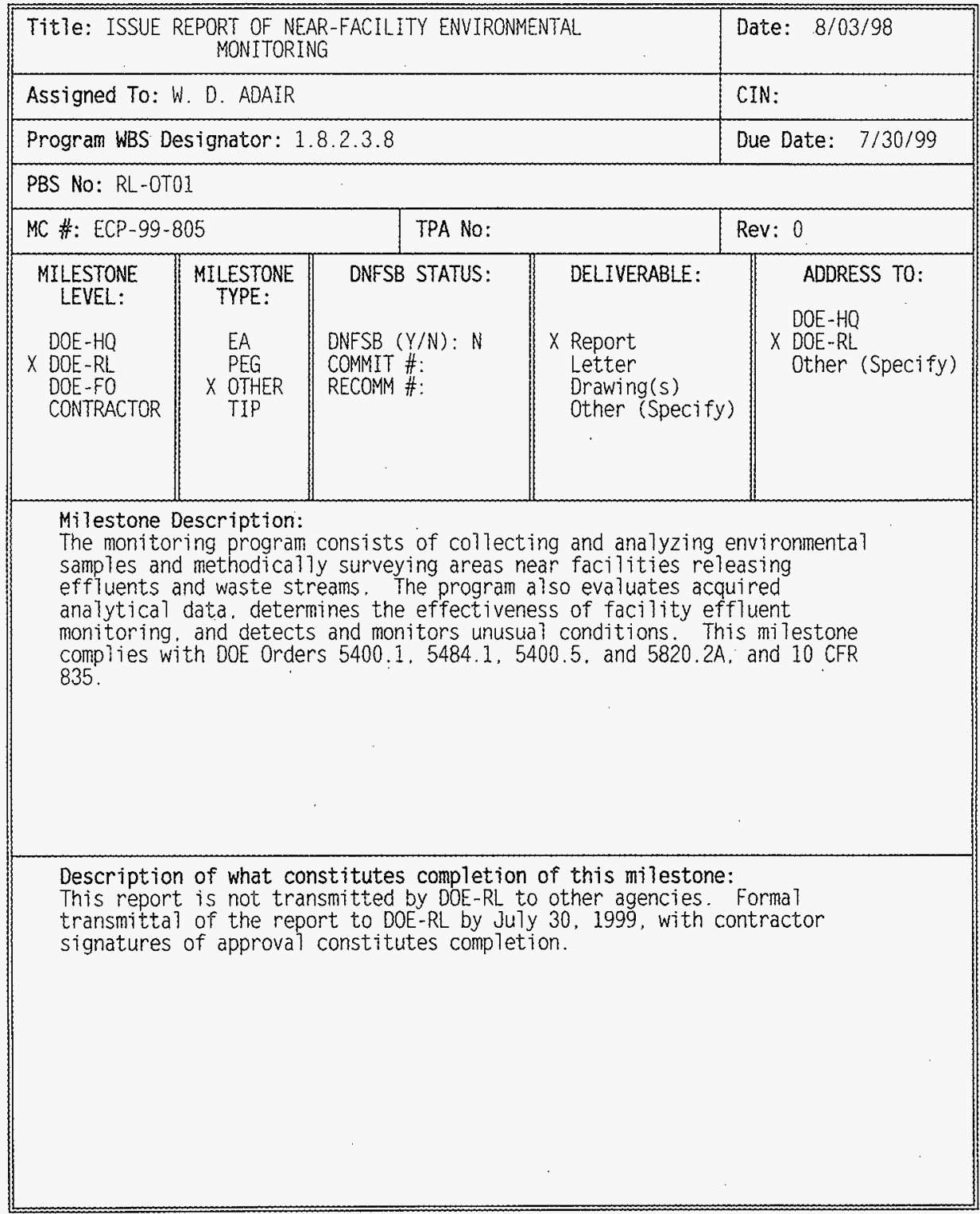


HNF-SP-1112, Rev. 4

PHMC

MILESTONE DESCRIPTION SHEET

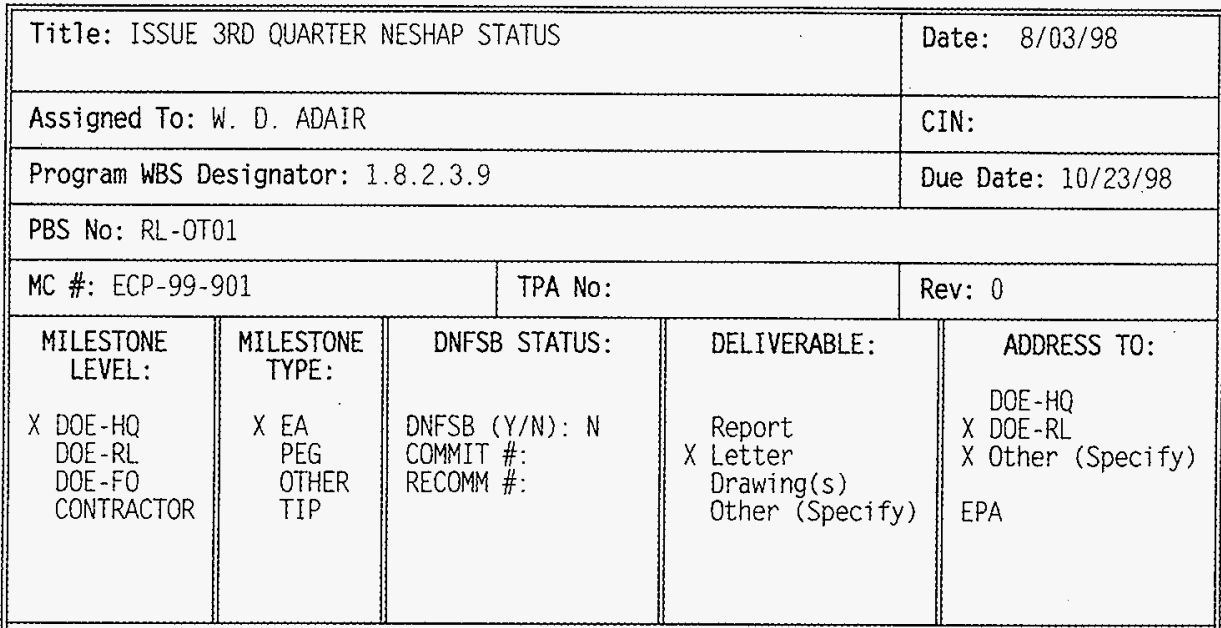

Milestone Description:

This milestone is a quarterly report on the progress made towards

satisfying the FFCA requirements. This quarterly report is required as an update on Hanford Site activities performed in support of the FFCA.

Description of what constitutes completion of this milestone:

This milestone is completed by delivering the report to DOE-RL and providing a telefax transmittal to EPA. The report is subsequently transmitted by DOE-RL to the regulators by $11 / 06 / 98$. 


\begin{tabular}{|c|c|c|c|c|}
\hline \multicolumn{4}{|c|}{ Title: ISSUE 4TH QUARTER NESHAP STATUS } & Date: $8 / 03 / 98$ \\
\hline \multicolumn{4}{|c|}{ Assigned To: W. D. ADAIR } & CIN: \\
\hline \multicolumn{4}{|c|}{ Program WBS Designator: 1.8.2.3.9 } & Due Date: $1 / 22 / 99$ \\
\hline \multicolumn{5}{|c|}{ PBS No: RL-0T01 } \\
\hline \multicolumn{2}{|c|}{ MC \#: ECP-99-902 } & TPA No: & & Rev: 0 \\
\hline $\begin{array}{l}\text { MILESTONE } \\
\text { LEVEL: } \\
\times \text { DOE-HQ } \\
\text { DOE-RL } \\
\text { DOE-FO } \\
\text { CONTRACTOR }\end{array}$ & $\begin{array}{l}\text { MILESTONE } \\
\text { TYPE: } \\
\times \text { EA } \\
\text { PEG } \\
\text { OTHER } \\
\text { TIP }\end{array}$ & $\begin{array}{l}\text { DNFSB STATUS: } \\
\text { DNFSB }(Y / N): N \\
\text { COMMIT \#: } \\
\text { RECOMM \#: }\end{array}$ & $\begin{array}{l}\text { DELIVERABLE: } \\
\\
\text { Report } \\
\times \text { Letter } \\
\text { Drawing(s) } \\
\text { Other (Specify) }\end{array}$ & $\begin{array}{l}\text { ADDRESS TO: } \\
\text { DOE-HQ } \\
\times \text { DOE-RL (Specify) } \\
x \text { Other (Spes } \\
\text { EPA }\end{array}$ \\
\hline \multicolumn{5}{|c|}{$\begin{array}{l}\text { Milestone Description: } \\
\text { This milestone is a quarterly report on the progress made towards } \\
\text { satisfying the FFCA requirements. This quarterly report is required as an } \\
\text { update on Hanford Site activities performed in support of the FFCA. }\end{array}$} \\
\hline \multicolumn{5}{|c|}{$\begin{array}{l}\text { Description of what constitutes completion of this milestone: } \\
\text { This milestone is completed by delivering the report to DOE-RL and } \\
\text { providing a telefax transmittal to EPA. The report is subsequently } \\
\text { transmitted by DOE-RL to the regulators by } 02 / 05 / 99 \text {. }\end{array}$} \\
\hline
\end{tabular}




\section{HNF-SP-1112, Rev. 4 \\ PHMC \\ MILESTONE DESCRIPTION SHEET}

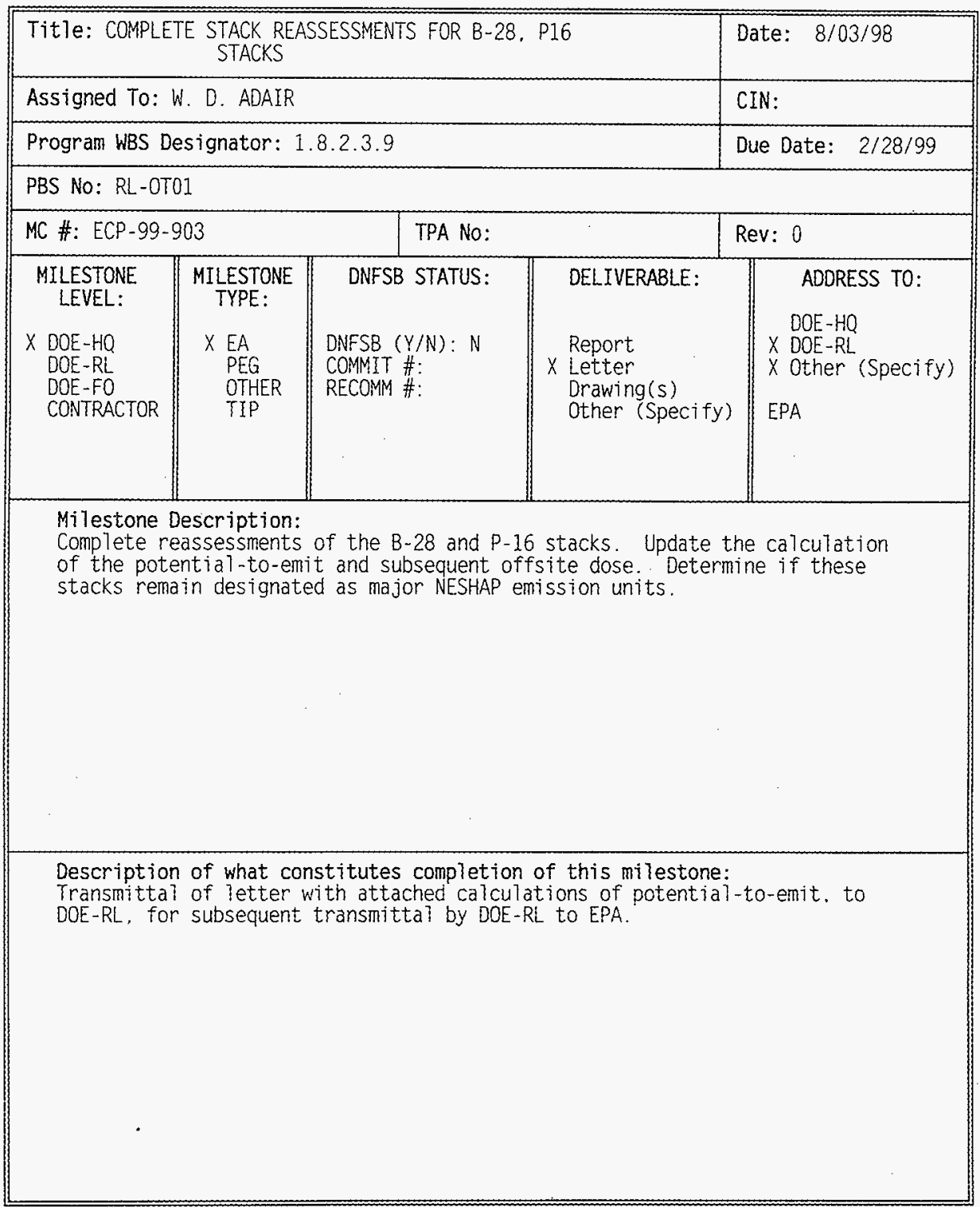




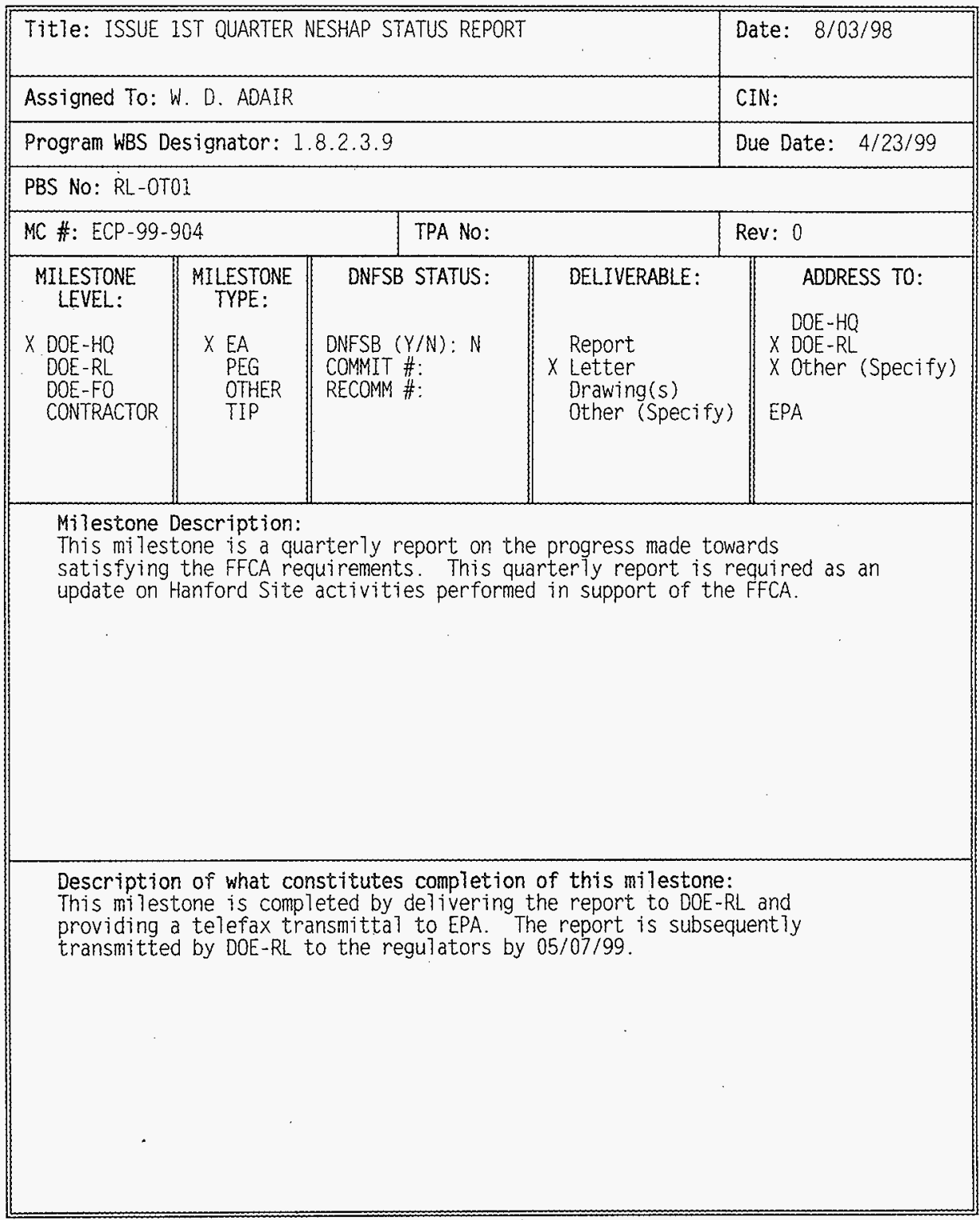


PHMC

\section{MILESTONE DESCRIPTION SHEET}

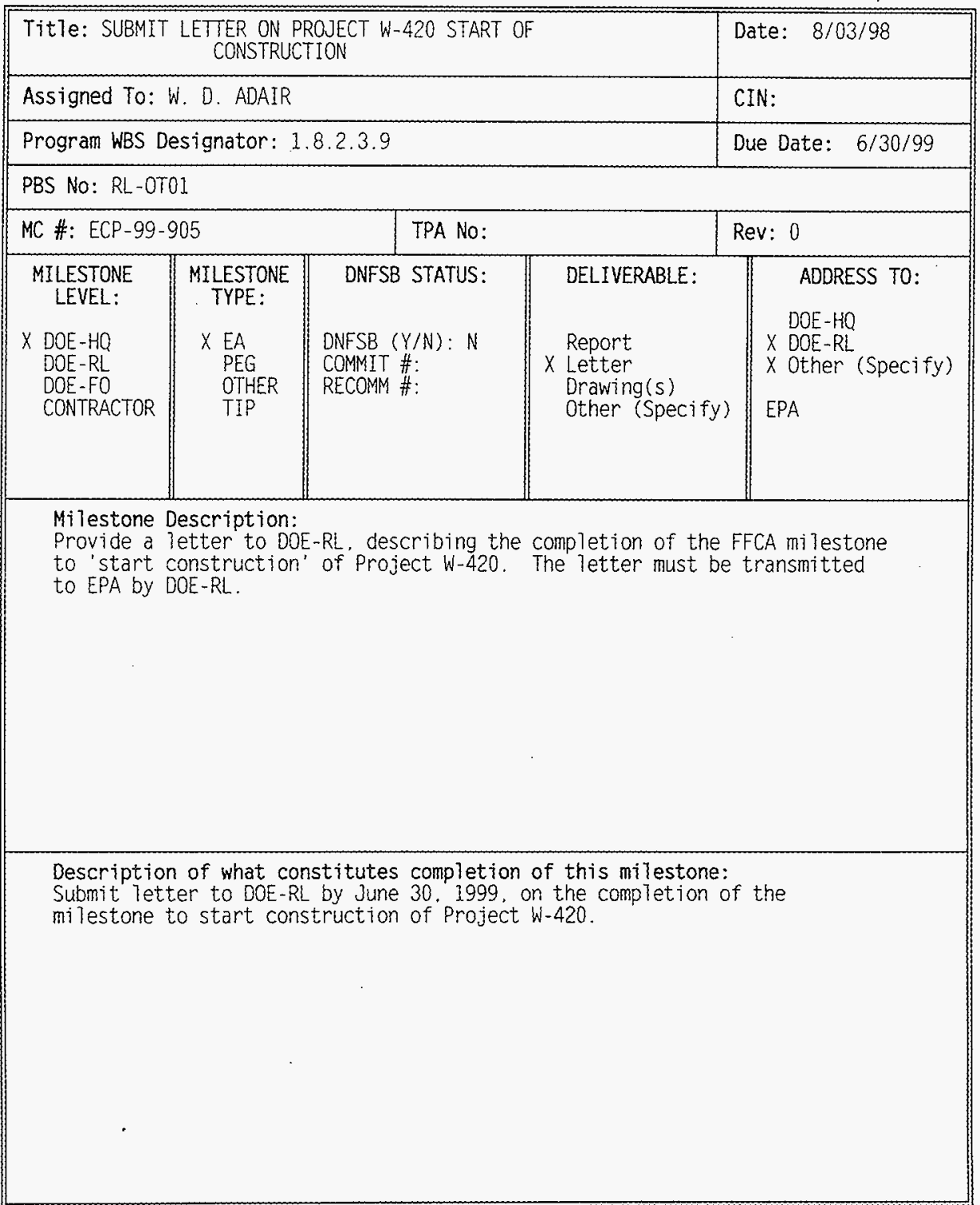




\section{PHMC}

MILESTONE DESCRIPTION SHEET

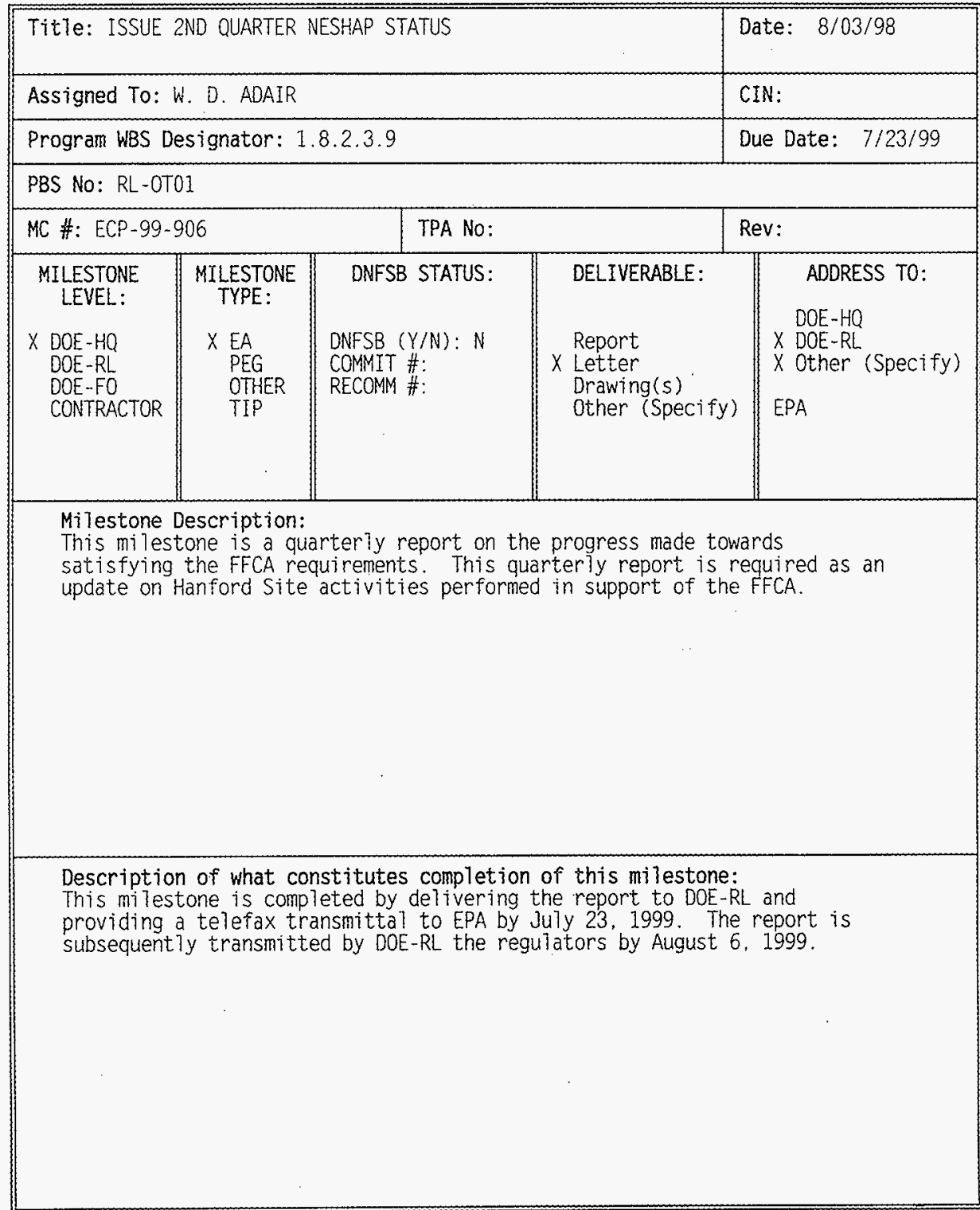




\section{PHMC}

\section{MILESTONE DESCRIPTION SHEET}

\begin{tabular}{|c|c|c|c|c|}
\hline \multicolumn{4}{|c|}{ Title: CONCRETE PAD FOR STACK 296-A-25 (244-A DCRT) } & Date: $8 / 04 / 98$ \\
\hline \multicolumn{4}{|c|}{ Assigned To: W. D. ADAIR } & CIN: \\
\hline \multicolumn{4}{|c|}{ Program WBS Designator: 1.8.2.3.11 } & Due Date: 6/30/99 \\
\hline \multicolumn{5}{|c|}{ PBS No: RL-0T01 } \\
\hline \multicolumn{2}{|c|}{ MC \#: ECP-99-111 } & TPA No: & & Rev: 0 \\
\hline $\begin{array}{l}\text { MILESTONE } \\
\text { LEVEL: } \\
\times \text { DOE-HQ } \\
\text { DOE-RL } \\
\text { DOE-FO } \\
\text { CONTRACTOR }\end{array}$ & $\begin{array}{l}\text { MILESTONE } \\
\text { TYPE: } \\
X \text { EA } \\
\text { PEG } \\
\text { OTHER } \\
\text { TIP }\end{array}$ & $\begin{array}{l}\text { DNFSB STATUS: } \\
\text { DNFSB }(Y / N): N \\
\text { COMMIT \#: } \\
\text { RECOMM \#: }\end{array}$ & $\begin{array}{l}\text { DELIVERABLE: } \\
\\
\text { Report } \\
\times \text { Letter } \\
\text { Orawing(s) } \\
\text { Other (Specify) }\end{array}$ & $\begin{array}{l}\text { ADDRESS TO: } \\
\text { DOE-HO } \\
\times \text { DOE-RL } \\
\text { Other (Specify) }\end{array}$ \\
\hline \multicolumn{5}{|c|}{$\begin{array}{l}\text { Milestone Description: } \\
\text { Place ancillary concrete pad at } 244-A \text { DCRT in support of the monitoring } \\
\text { upgrade on stack } 296-A-25 \text { to meet one of the six legally enforceable } \\
\text { Federal Facility Compliance Agreement milestones. }\end{array}$} \\
\hline \multicolumn{5}{|c|}{$\begin{array}{l}\text { Description of what constitutes completion of this milestone: } \\
\text { Letter report to DoE-RL by June } 30 \text {. } 1999 \text {. that documents the completion of } \\
\text { the placement of the pad at } 244-A \text { OCRT. }\end{array}$} \\
\hline
\end{tabular}




\section{PHMC}

MILESTONE DESCRIPTION SHEET

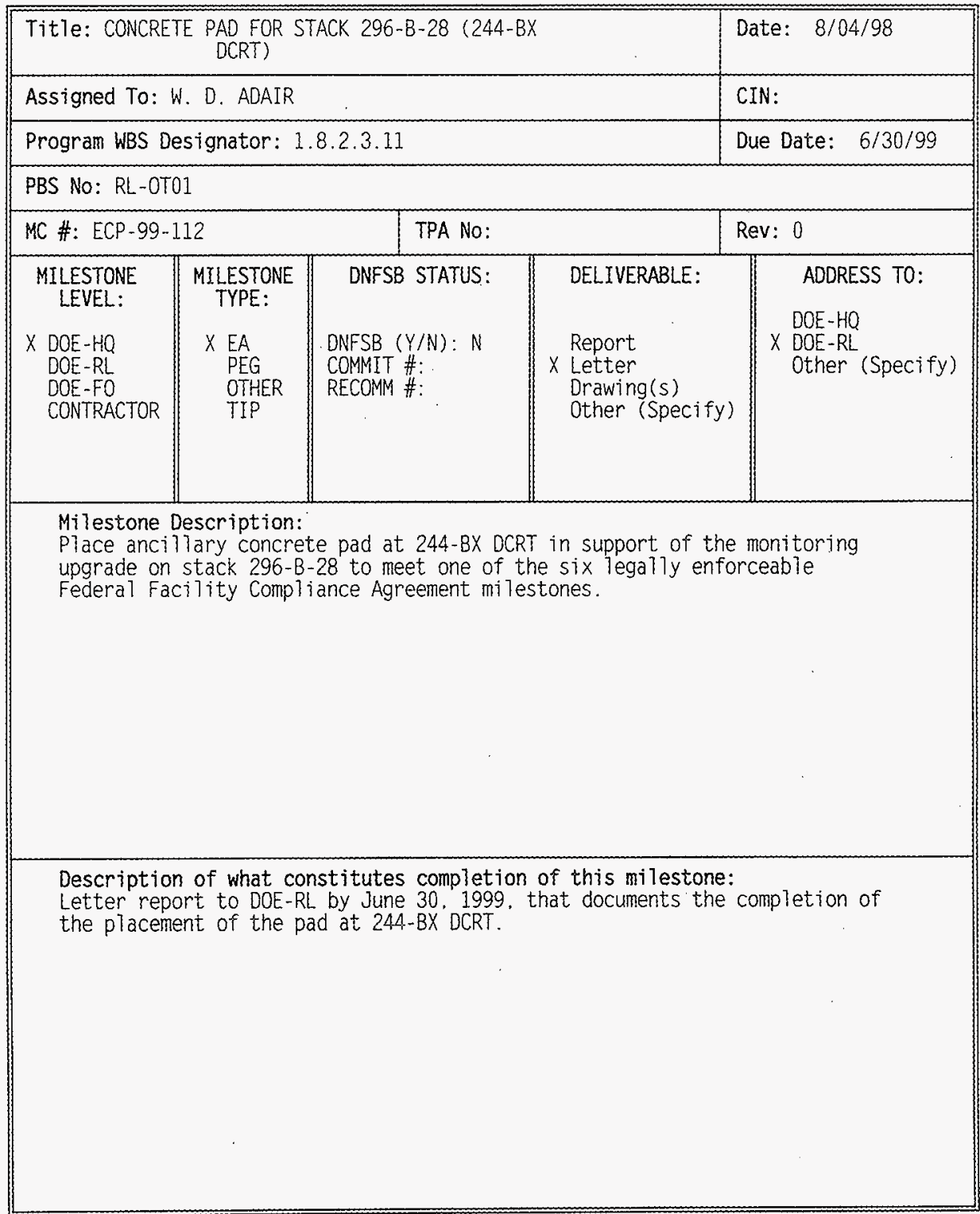




\section{PHMC}

\section{MILESTONE DESCRIPTION SHEET}

\begin{tabular}{|c|c|c|c|c|}
\hline \multicolumn{4}{|c|}{$\begin{array}{l}\text { Title: CONCRETE PAD FOR STACK 296-C-5 (244-CR } \\
\text { VAULT) }\end{array}$} & Date: $8 / 04 / 98$ \\
\hline \multicolumn{4}{|c|}{ Assigned To: $W$. D. ADAIR } & CIN: \\
\hline \multicolumn{4}{|c|}{ Program WBS Designator: 1.8.2.3.11 } & Due Date: $6 / 30 / 99$ \\
\hline \multicolumn{5}{|c|}{ PBS No: RL-0T01 } \\
\hline \multicolumn{2}{|c|}{ MC \#: ECP-99-113 } & \multicolumn{2}{|l|}{ TPA No: } & Rev: 0 \\
\hline $\begin{array}{l}\text { MILESTONE } \\
\text { LEVEL: } \\
\times \text { DOE-HQ } \\
\text { DOE-RL } \\
\text { DOE-FO } \\
\text { CONTRACTOR }\end{array}$ & $\begin{array}{l}\text { MILESTONE } \\
\text { TYPE: } \\
\times \text { EA } \\
\text { PEG } \\
\text { OTHER } \\
\text { TIP }\end{array}$ & $\begin{array}{l}\text { DNFSB STATUS: } \\
\text { DNFSB }(Y / N): N \\
\text { COMMIT \#: } \\
\text { RECOMM \#: }\end{array}$ & $\begin{array}{l}\text { DELIVERABLE: } \\
\text { Report } \\
\times \text { Letter } \\
\text { Drawing (s) } \\
\text { Other (Specify) }\end{array}$ & $\begin{array}{l}\text { ADORESS TO: } \\
\text { DOE-HQ } \\
\times \text { DOE-RL } \\
\text { Other (Specify) }\end{array}$ \\
\hline
\end{tabular}

Milestone Description:

Place ancillary concrete pad at 244-CR Vault in support of the monitoring upgrade on stack 296-C-5 to meet one of the six legally enforceable Federal Facility Compliance Agreement milestones.

Description of what constitutes completion of this milestone:

Letter report to DOE-RL by June 30. 1999, that documents the completion of the placement of the pad at 244-CR Vault. 
HNF-SP-1112, Rev. 4

PHMC

MILESTONE DESCRIPTION SHEET

\begin{tabular}{|c|c|c|c|c|}
\hline \multicolumn{4}{|c|}{$\begin{array}{c}\text { Title: CONCRETE PAD FOR STACK 296-P-16 (244-C TANK } \\
\text { FARM) }\end{array}$} & Date: $8 / 04 / 98$ \\
\hline \multicolumn{4}{|c|}{ Assigned To: $W$. D. ADAIR } & CIN: \\
\hline \multicolumn{4}{|c|}{ Program WBS Designator: 1.8.2.3.11 } & Due Date: $6 / 30 / 99$ \\
\hline \multicolumn{5}{|c|}{ PBS NO: RL-0T01 } \\
\hline \multicolumn{2}{|c|}{ MC \#: ECP-99-114 } & TPA No: & & Rev: 0 \\
\hline $\begin{array}{l}\text { MILESTONE } \\
\text { LEVEL: } \\
\text { X DOE-HQ } \\
\text { DOE-RL } \\
\text { DOE-FO } \\
\text { CONTRACTOR }\end{array}$ & $\begin{array}{l}\text { MILESTONE } \\
\text { TYPE: } \\
\times \text { EA } \\
\text { PEG } \\
\text { OTHER } \\
\text { TIP }\end{array}$ & $\begin{array}{l}\text { DNFSB STATUS: } \\
\text { DNFSB }(Y / N): N \\
\text { COMMIT \#: } \\
\text { RECOMM \#: }\end{array}$ & $\begin{array}{l}\text { DELIVERABLE: } \\
\text { Report } \\
\times \text { Letter } \\
\text { Drawing(s) } \\
\text { Other (Specify) }\end{array}$ & $\begin{array}{l}\text { ADDRESS TO: } \\
\text { DOE-HO } \\
\times \text { DOE-RL } \\
\text { Other (Specify) }\end{array}$ \\
\hline \multicolumn{5}{|c|}{$\begin{array}{l}\text { Milestone Description: } \\
\text { Place ancillary concrete pad at } 241-C \text { Tank Farm in support of the } \\
\text { monitoring upgrade on stack } 296-16 \text { - } 16 \text { to meet one of the six legally } \\
\text { enforceable Federa? Facility Compliance Agreement milestones. }\end{array}$} \\
\hline \multicolumn{5}{|c|}{$\begin{array}{l}\text { Description of what constitutes completion of this milestone: } \\
\text { Letter report to DOE-RL by June } 30 \text {, } 1999 \text {, that documents the completion of } \\
\text { the placement of the pad at } 241-C \text { Tank Farm. }\end{array}$} \\
\hline
\end{tabular}


PHMC

MILESTONE DESCRIPTION SHEET

\begin{tabular}{|c|c|c|c|c|}
\hline \multicolumn{4}{|c|}{ TitTe: CONCRETE PAD FOR STACK 296-S-22 (244-S DCRT) } & Date: $8 / 04 / 98$ \\
\hline \multicolumn{4}{|c|}{ Assigned To: W. D. ADAIR } & CIN: \\
\hline \multicolumn{4}{|c|}{ Program WBS Designator: 1.8 .2 .3 .11} & Due Date: $6 / 30 / 99$ \\
\hline \multicolumn{5}{|c|}{ PBS No: RL-OT01 } \\
\hline \multicolumn{2}{|c|}{ MC \#: ECP-99-115 } & TPA No: & & Rev: 0 \\
\hline $\begin{array}{l}\text { MILESTONE } \\
\text { LEVEL: } \\
\text { X DOE-HQ } \\
\text { DOE-RL } \\
\text { DOE-FO } \\
\text { CONTRACTOR }\end{array}$ & $\begin{array}{l}\text { MILESTONE } \\
\text { TYPE: } \\
\times \text { EA } \\
\text { PEG } \\
\text { OTHER } \\
\text { TIP }\end{array}$ & $\begin{array}{l}\text { DNFSB STATUS: } \\
\text { DNFSB }(Y / N): N \\
\text { COMMIT \#: } \\
\text { RECOMM \#: }\end{array}$ & $\begin{array}{l}\text { DELIVERABLE: } \\
\text { Report } \\
\times \text { Letter } \\
\text { Drawing(s) } \\
\text { Other (Specify) }\end{array}$ & $\begin{array}{l}\text { ADDRESS TO: } \\
\text { DOE-HO } \\
\times \text { DOE-RL } \\
\text { Other (Specify) }\end{array}$ \\
\hline \multirow{2}{*}{\multicolumn{5}{|c|}{$\begin{array}{l}\text { Milestone Description: } \\
\text { Please ancillary concrete pad at } 244-S \text { DCRT in support of the monitoring } \\
\text { upgrade on stack } 296-5-22 \text { to meet one of the six legally enforceable } \\
\text { Federal Facility CompTiance Agreement milestones. }\end{array}$}} \\
\hline & & & & \\
\hline \multicolumn{5}{|c|}{$\begin{array}{l}\text { Description of what constitutes completion of this milestone: } \\
\text { Letter report to DoE-RL by June } 30 \text {, } 1999 \text {. that documents the completion of } \\
\text { the placement of the pad at } 244-S \text { DCRT. }\end{array}$} \\
\hline
\end{tabular}




\section{PHMC}

MILESTONE DESCRIPTION SHEET

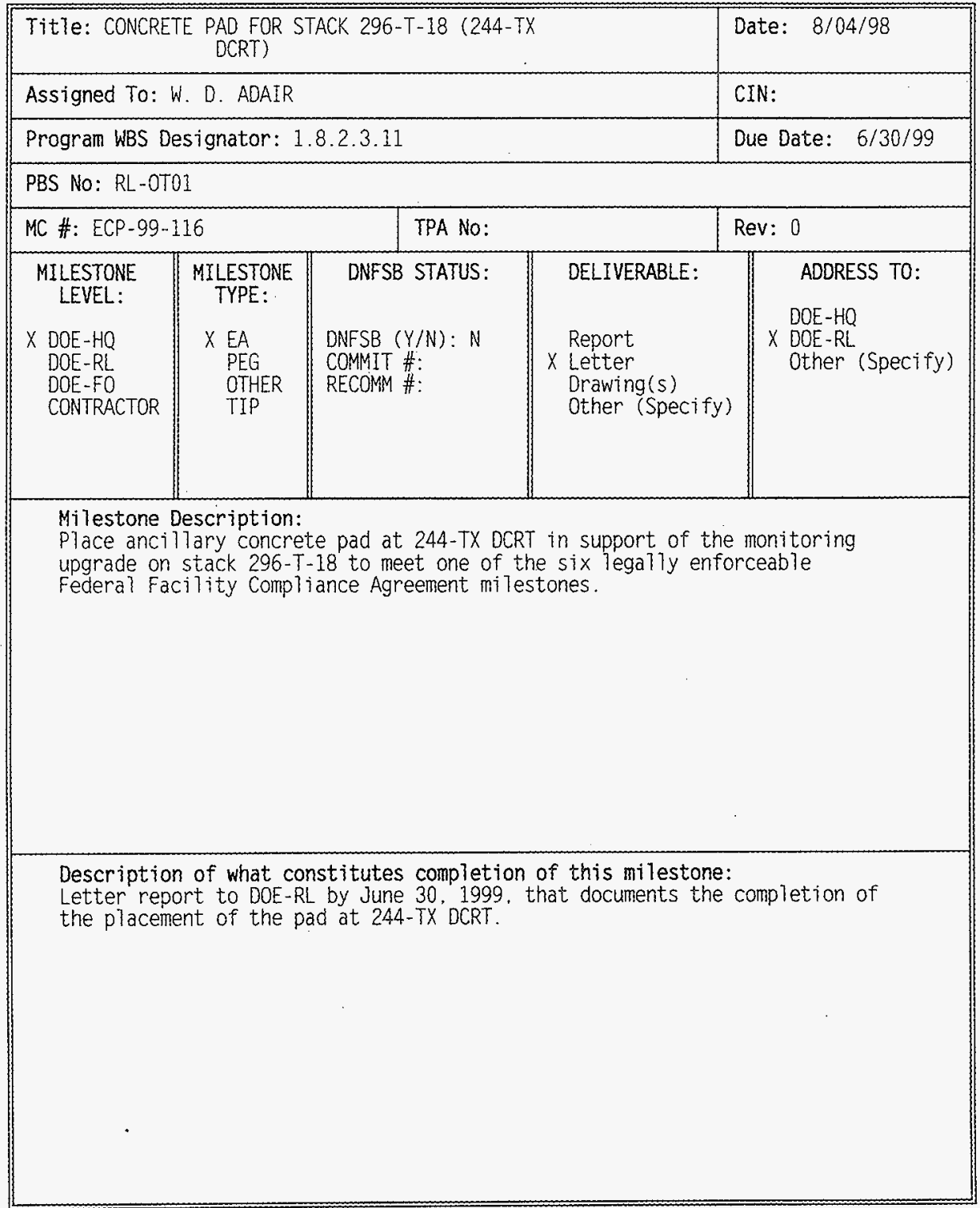




\section{Milestone Description Sheets - Out-years}

One set of milestone description sheets for lifecycle are housed in the CMM. Since most milestones in the ECP are perennial, one set of MDS is representative of all the lifecycle deliverables which is through the year 2046. A representative milestone description sheet is provided for each EA-type and Other-type milestone. All EA-type milestones are HQ-level, while the Other-type milestones are RL-fevel. Please see the lifecycle MDS on the following pages. 
PHMC

MILESTONE DESCRIPTION SHEET

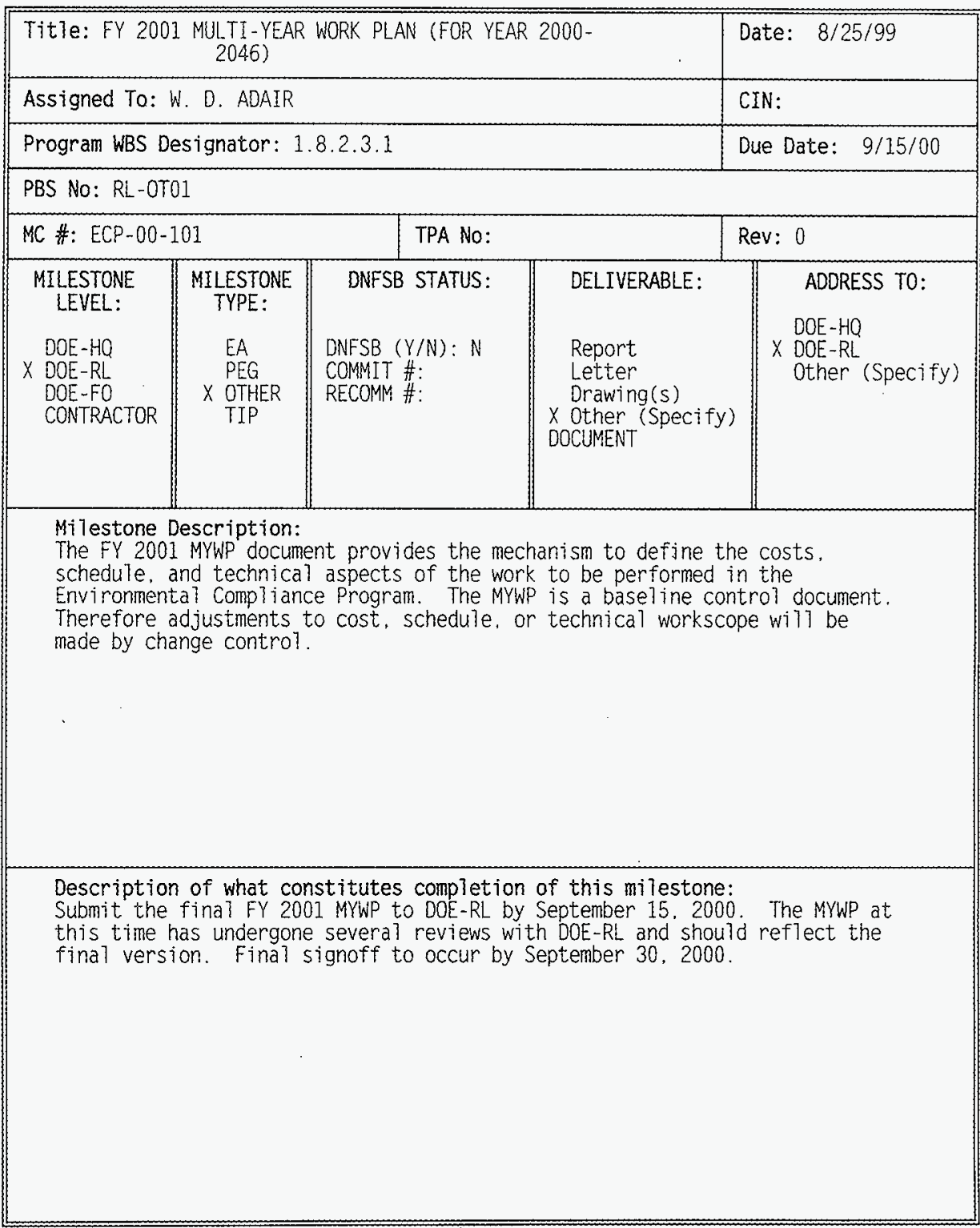


PHMC

MILESTONE DESCRIPTION SHEET

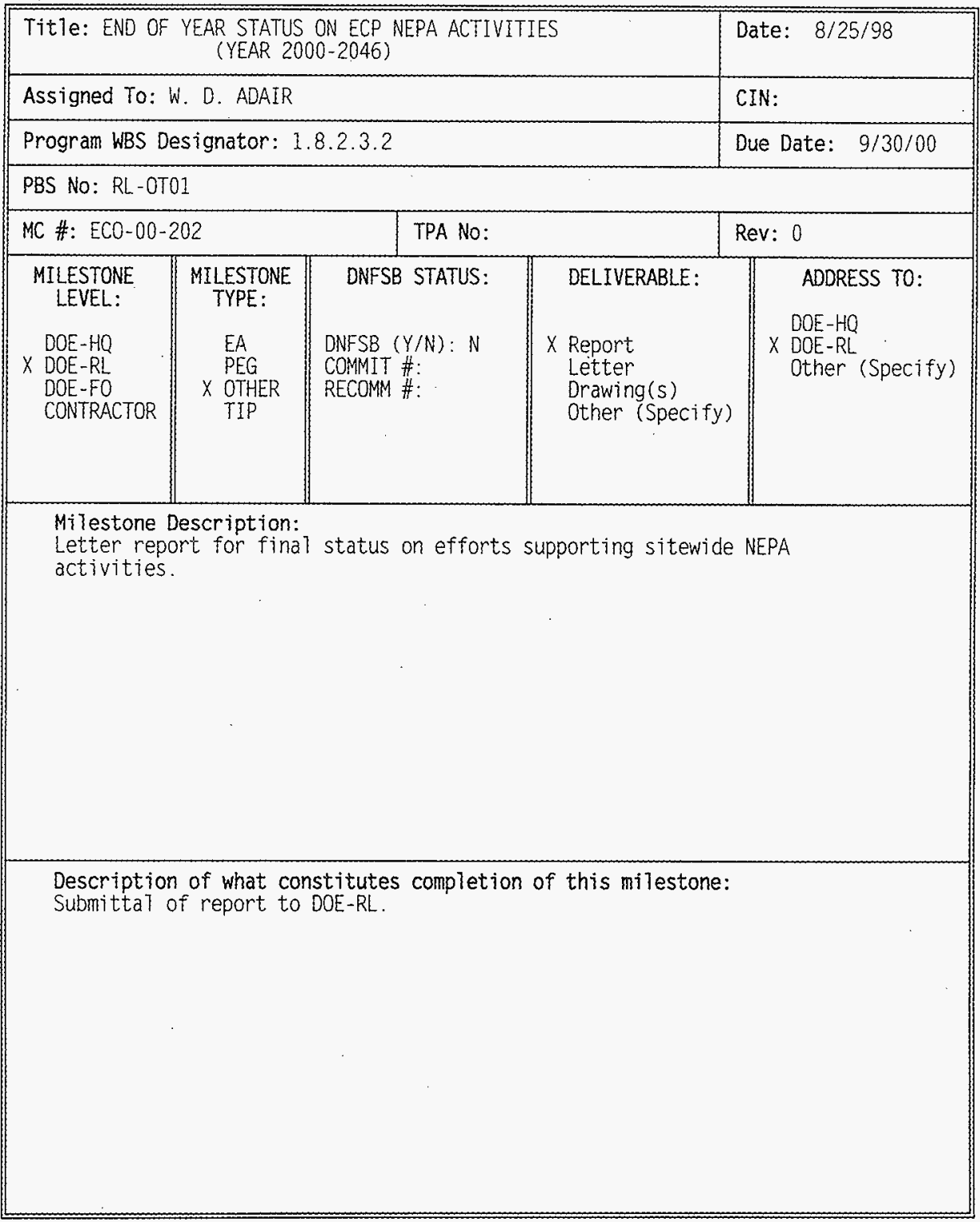


HNF-SP-1112, Rev. 4

\section{PHMC}

\section{MILESTONE DESCRIPTION SHEET}

\begin{tabular}{|c|c|c|c|c|}
\hline \multicolumn{4}{|c|}{$\begin{array}{l}\text { Title: ISSUE UPDATED NEPA SOURCE GUIDE (FOR FY } 2000 \\
\qquad-2046 \text { ) }\end{array}$} & Date: $8 / 25 / 98$ \\
\hline \multicolumn{4}{|c|}{ Assigned To: WD ADAIR } & CIN: \\
\hline \multicolumn{4}{|c|}{ Program WBS Designator: $1.8 \cdot 2.3 .2$} & Due Date: $9 / 30 / 00$ \\
\hline \multicolumn{5}{|c|}{ PBS No: RL-0T01 } \\
\hline \multicolumn{2}{|c|}{ MC \#: ECP-00-203 } & TPA NO: & & Rev: 0 \\
\hline $\begin{array}{l}\text { MILESTONE } \\
\text { LEVEL: } \\
\text { DOE-HQ } \\
\times \text { DOE-RL } \\
\text { DOE-FO } \\
\text { CONTRACTOR }\end{array}$ & $\begin{array}{l}\text { MILESTONE } \\
\text { TYPE: } \\
\\
\text { EA } \\
\text { PEG } \\
\times \text { OTHER } \\
\text { TIP }\end{array}$ & $\begin{array}{l}\text { DNFSB STATUS: } \\
\text { DNFSB }(Y / N): N \\
\text { COMMIT \#: } \\
\text { RECOMM \#: }\end{array}$ & $\begin{array}{l}\text { DELIVERABLE: } \\
\text { X Report } \\
\text { Letter } \\
\text { Drawing(s) } \\
\text { Other (Specify) }\end{array}$ & $\begin{array}{l}\text { ADDRESS TO: } \\
\text { DOE-HO } \\
\times \text { DOE-RL } \\
\text { Other (Specify) }\end{array}$ \\
\hline \multicolumn{5}{|c|}{$\begin{array}{l}\text { Milestone Description: } \\
\text { Update NEPA Source Guide information and issue revised reference. Place } \\
\text { Source Guide information on Hanford Intranet. }\end{array}$} \\
\hline \multicolumn{5}{|c|}{$\begin{array}{l}\text { Description of what constitutes completion of this milestone: } \\
\text { Submittâ of updated NEPA Source Guide to DOE-RL. }\end{array}$} \\
\hline
\end{tabular}




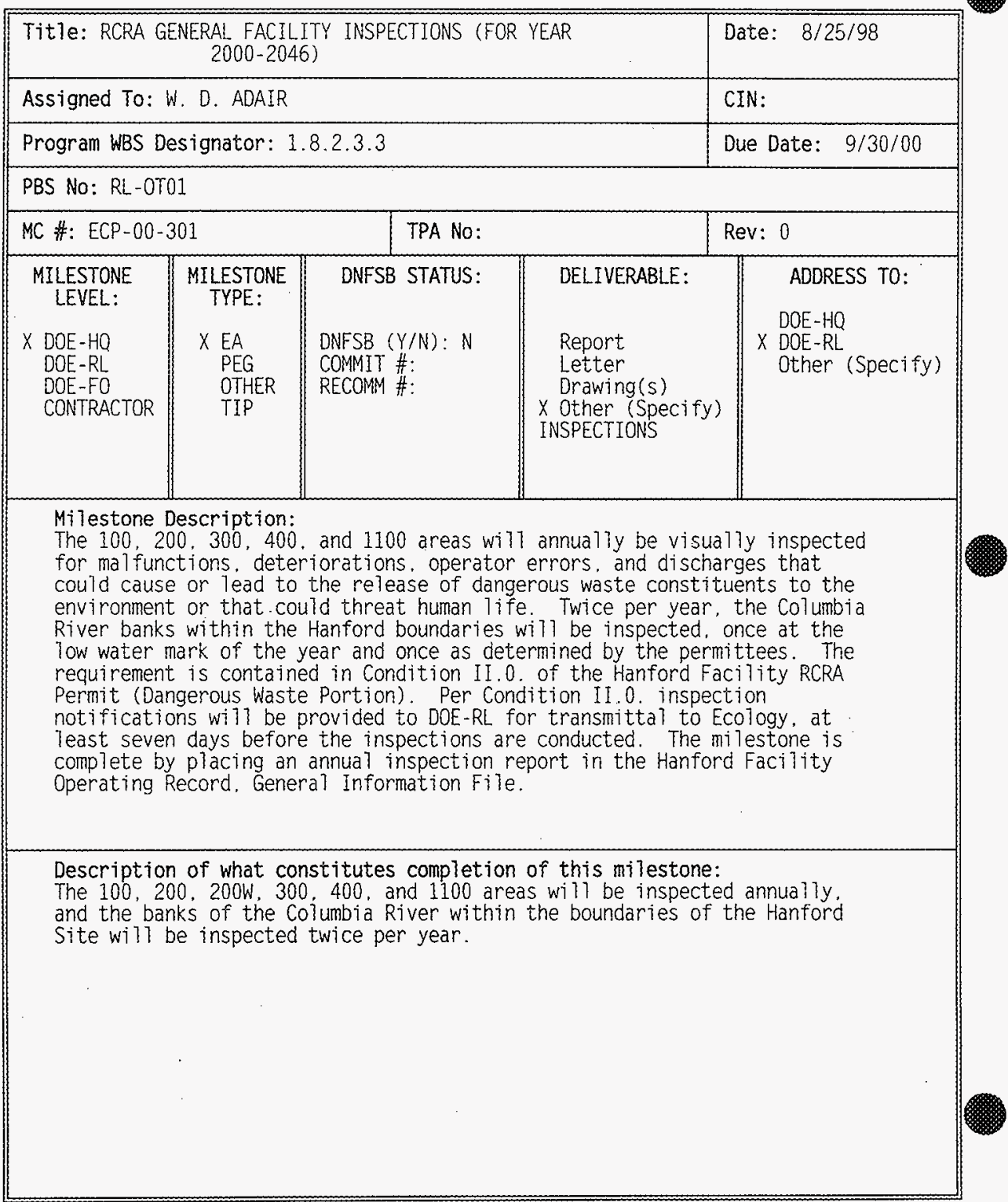


PHMC

\section{MILESTONE DESCRIPTION SHEET}

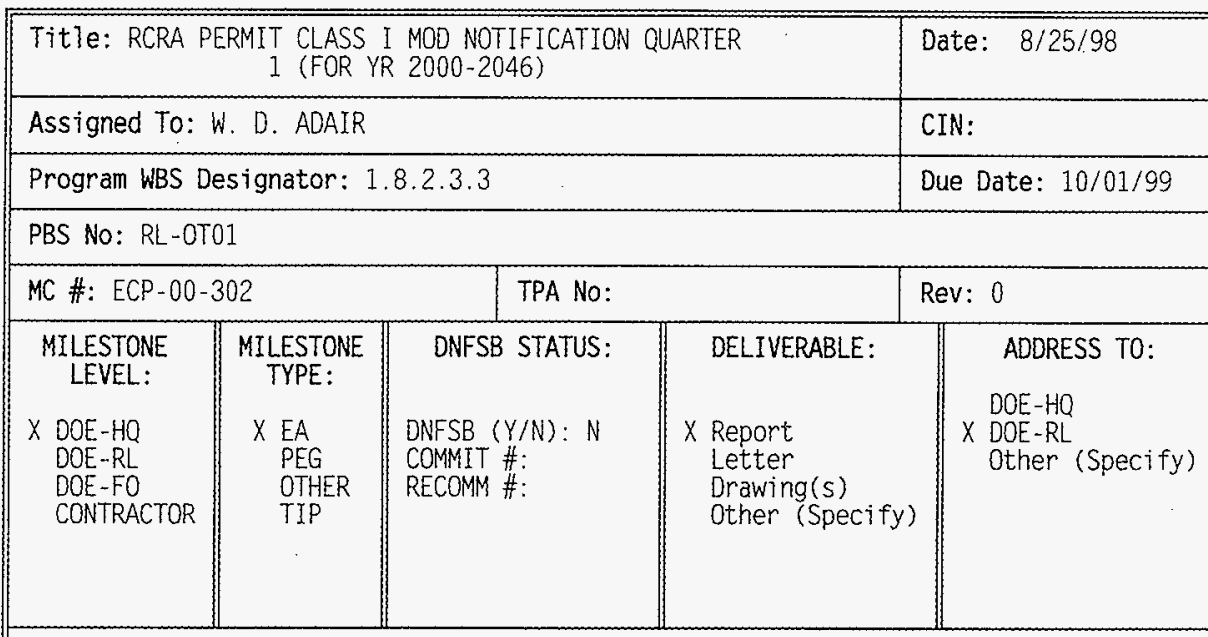

Milestone Description:

A quarterly notification report wi 11 be submitted to DOE-RL as required by WAC 173-303-830(4)(a)(i)(A) and Condition I.C.3 of the Hanford Facility RCRA Permit (Dangerous Waste Portion). The report is to provide information for all Class I Modifications put into effect at Contractor Cooperated TSD units during the reporting period. The report wil 1 include TSD unit information received in a complete form 30 days before the regulator due date.

Description of what constitutes completion of this milestone:

Submit quarterly Notification of Class I Modifications of the Hanford Faci7 ity RCRA Permit to DOE-RL by October 1. 1999 for transmittal by DOE-RL to Ecology October 9, 1999. 
PHMC

\section{MILESTONE DESCRIPTION SHEET}

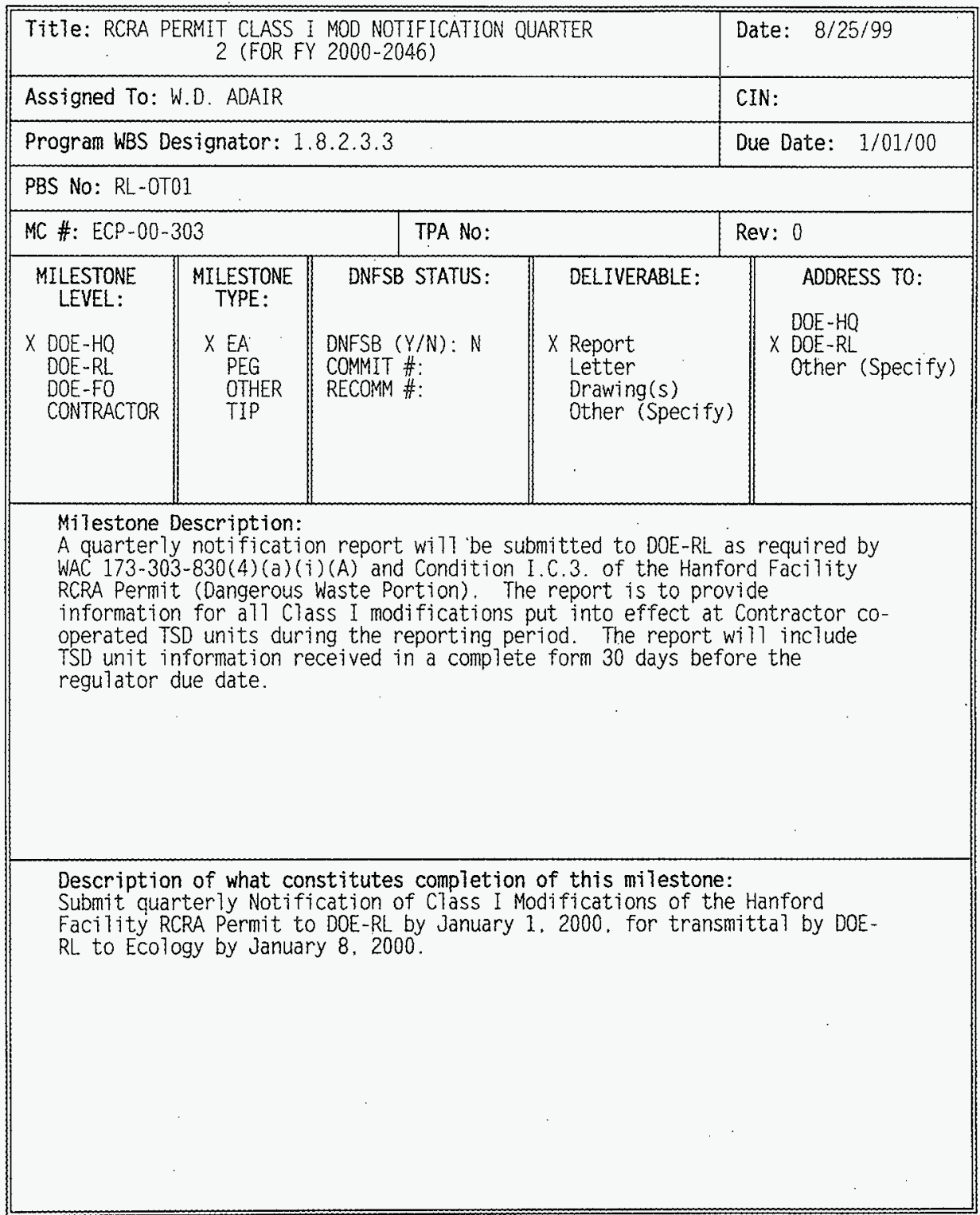




\section{PHMC}

\section{MILESTONE DESCRIPTION SHEET}

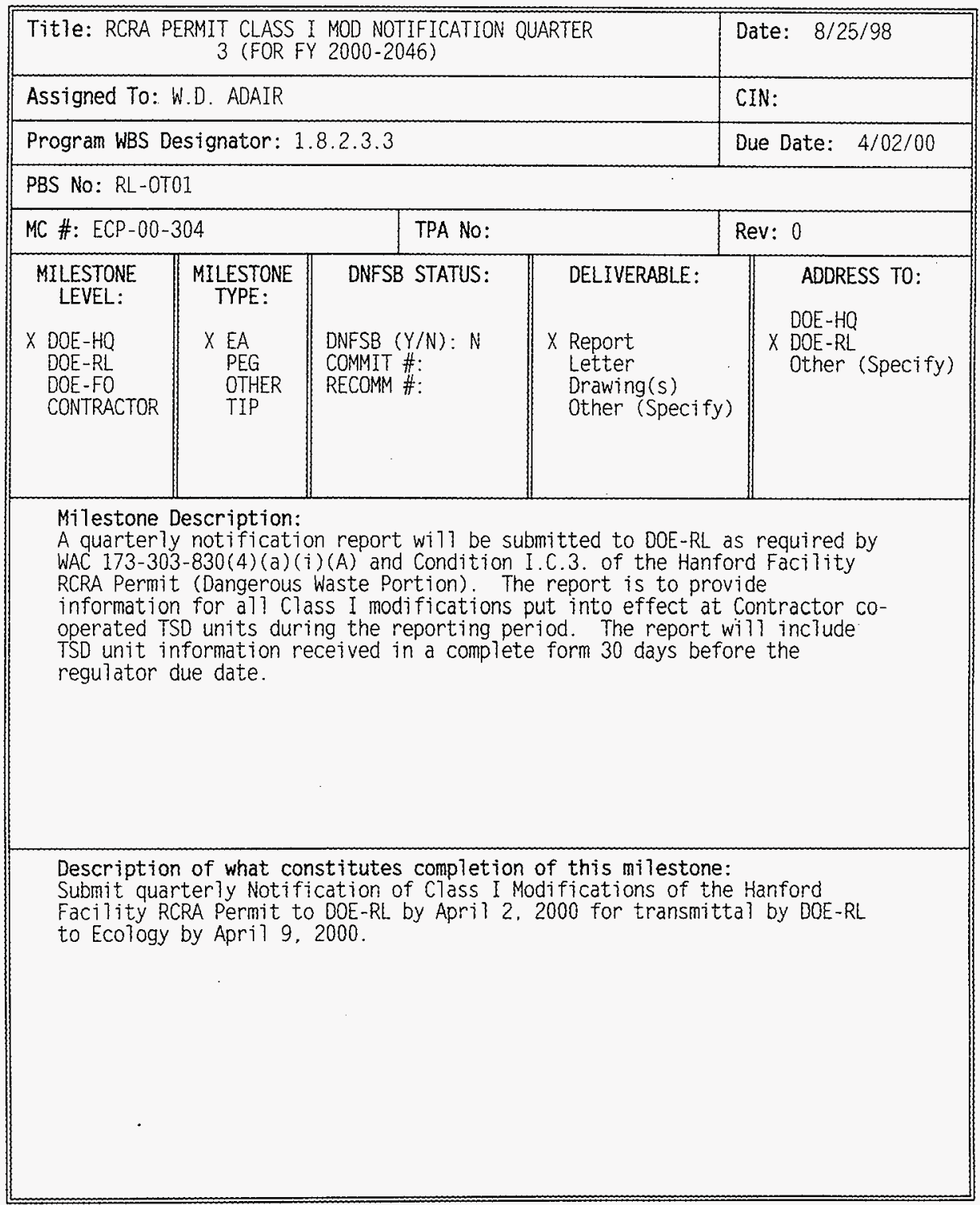


HNF-SP-1112, Rev. 4

\section{PHMC}

MILESTONE DESCRIPTION SHEET

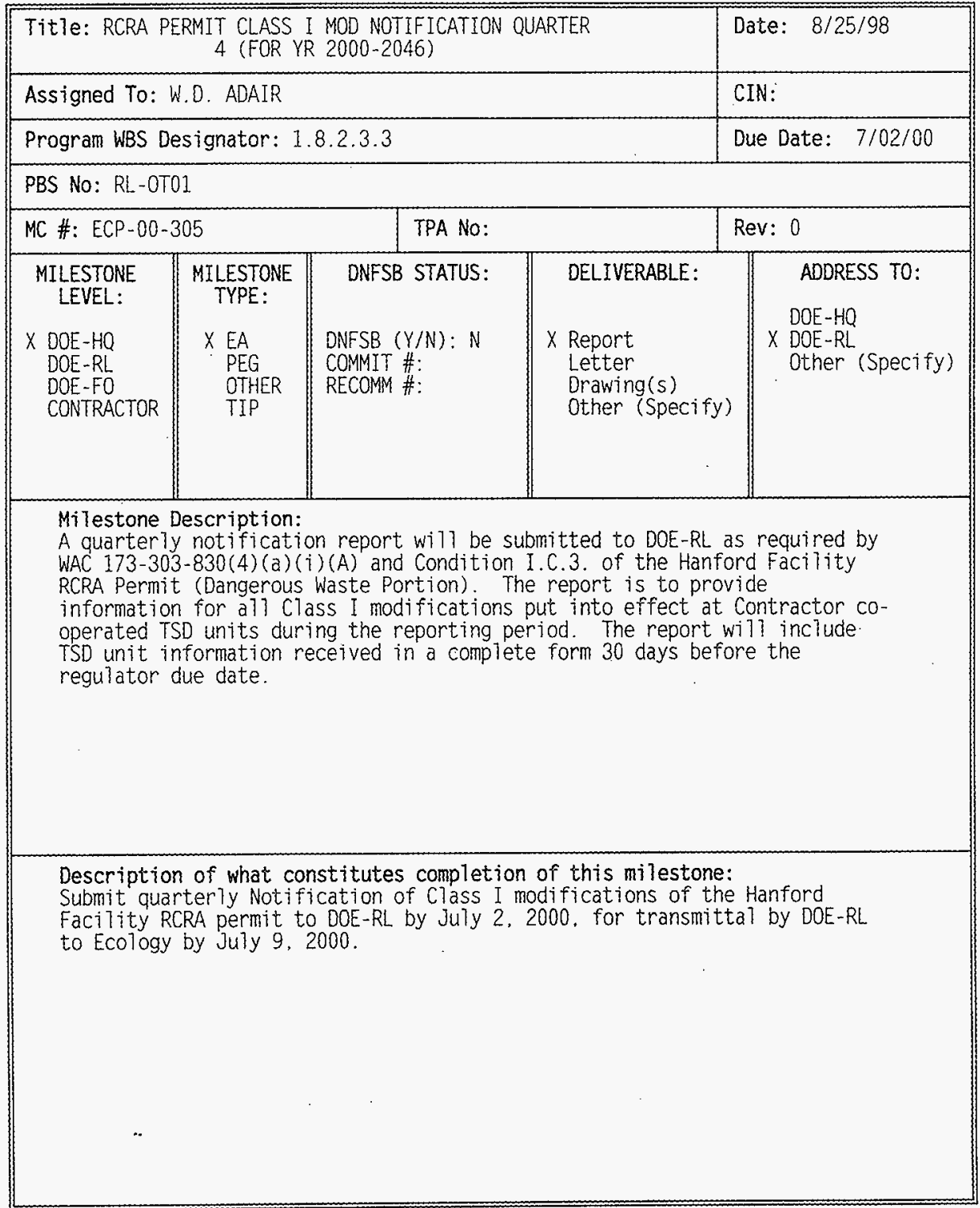


PHMC

MILESTONE DESCRIPTION SHEET

\begin{tabular}{|c|c|c|c|c|}
\hline \multicolumn{4}{|c|}{$\begin{array}{l}\text { Tit]e: ANNUAL ASBESTOS NOTIFICATION OF INTENT (FOR } \\
\text { YEAR 2000-2046) }\end{array}$} & Date: $8 / 25 / 98$ \\
\hline \multicolumn{4}{|c|}{ Assigned To: W.D. ADAIR } & CIN: \\
\hline \multicolumn{4}{|c|}{ Program WBS Designator: 1.8.2.3.3 } & Due Date: $12 / 31 / 99$ \\
\hline \multicolumn{5}{|c|}{ PBS NO: RL-OT01 } \\
\hline \multicolumn{2}{|c|}{ MC \#: ECP-00-306 } & TPA No: & & Rev: 0 \\
\hline $\begin{array}{l}\text { MILESTONE } \\
\text { LEVEL: } \\
\times \text { DOE-HQ } \\
\text { DOE-RL } \\
\text { DOE-FO } \\
\text { CONTRACTOR }\end{array}$ & $\begin{array}{l}\text { MILESTONE } \\
\text { TYPE: } \\
\times \text { EA } \\
\text { PEG } \\
\text { OTHER } \\
\text { TIP }\end{array}$ & $\begin{array}{l}\text { DNFSB STATUS: } \\
\text { ONFSB }(Y / N): N \\
\text { COMMIT \#: } \\
\text { RECOMM \#: }\end{array}$ & $\begin{array}{l}\text { DELIVERABLE: } \\
\text { X Report } \\
\text { Letter } \\
\text { Drawing(s) } \\
\text { Other (Specify) }\end{array}$ & $\begin{array}{l}\text { ADDRESS TO: } \\
\text { DOE-HO } \\
\times \text { DOE-RL } \\
X \text { Other (Specify) } \\
\text { BENTON COUNTY } \\
\text { CLEAN AIR AUTHOR }\end{array}$ \\
\hline \multicolumn{5}{|c|}{$\begin{array}{l}\text { Milestone Description: } \\
\text { FDH wil1 compile and send to the Benton Clean Air Authority the annual } \\
\text { asbestos notification of intent for Calendar year } 2000 \text { by December } 31 \text {, } \\
1999 \text {. }\end{array}$} \\
\hline \multicolumn{5}{|c|}{$\begin{array}{l}\text { Description of what constitutes completion of this milestone: } \\
\text { FDH will request information from site contractors. compile, and send to } \\
\text { the Benton Clean Air Authority the annual asbestos notification of intent } \\
\text { for Calendar Year } 2000 \text {. A copy to DOE-RL on the formal transmittal to the } \\
\text { Benton Clean Air Authority, due by December } 31.1999 \text {. constitutes } \\
\text { completion of this milestone. }\end{array}$} \\
\hline
\end{tabular}


PHMC

MILESTONE DESCRIPTION SHEET

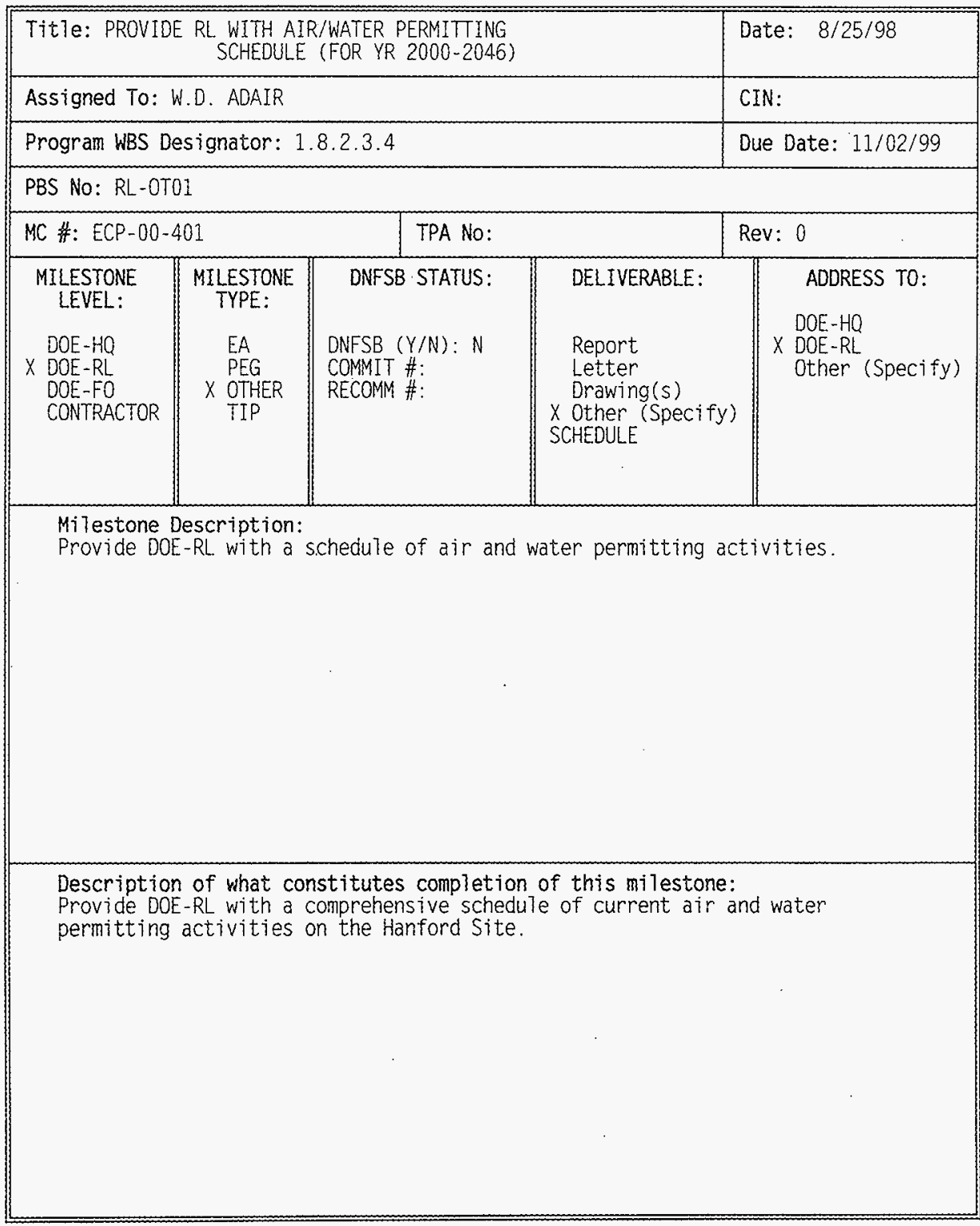




\section{PHMC}

MILESTONE DESCRIPTION SHEET

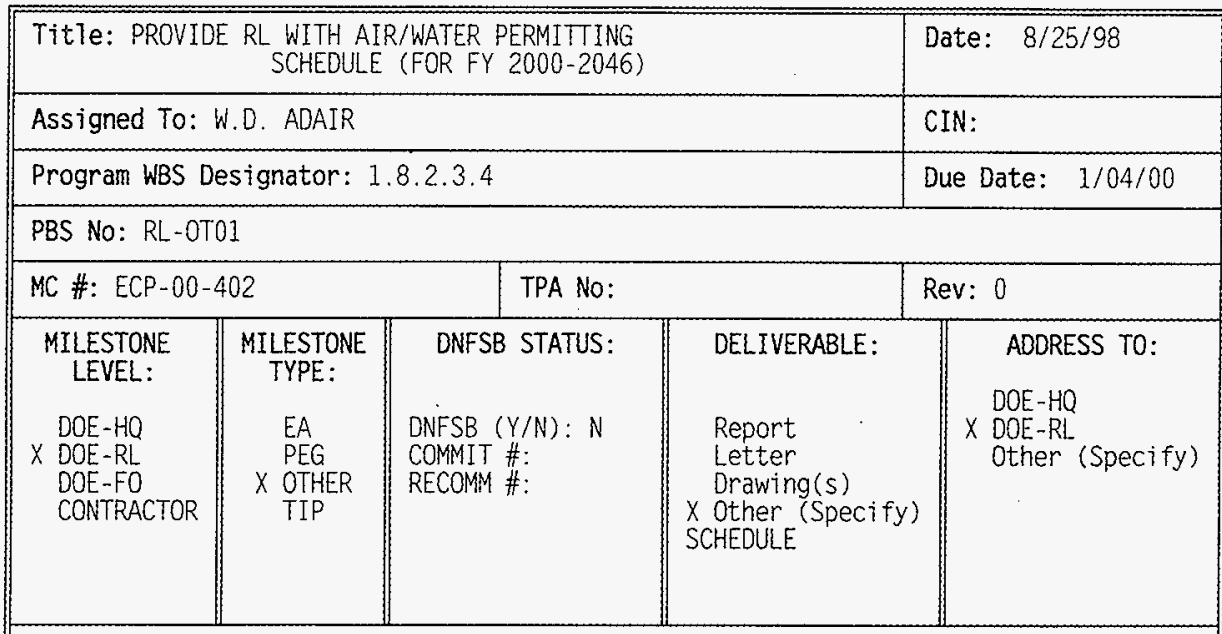

Milestone Description:

Provide DOE-RL with a schedule of air and water permitting activities.

Description of what constitutes completion of this milestone:

Provide DOE-RL with a comprehensive schedule of current air and water permitting activities on the Hanford Site. 
HNF-SP-1112, Rev. 4

\section{PHMC}

MILESTONE DESCRIPTION SHEET

\begin{tabular}{|c|c|c|c|c|}
\hline \multicolumn{4}{|c|}{$\begin{array}{r}\text { Title: PROVIDE RL WITH AIR/WATER PERMITTING } \\
\text { SCHEDULE (FOR FY 2000-2046) }\end{array}$} & Date: $8 / 25 / 98$ \\
\hline \multicolumn{4}{|c|}{ Assigned To: W.D. ADAIR } & CIN: \\
\hline \multicolumn{4}{|c|}{ Program WBS Designator: 1.8 .2 .3 .4} & Due Date: $3 / 01 / 00$ \\
\hline \multicolumn{5}{|c|}{ PBS No: RL-0T01 } \\
\hline \multicolumn{2}{|c|}{ MC \#: ECP-00-403 } & \multicolumn{2}{|l|}{ TPA No: } & Rev: 0 \\
\hline $\begin{array}{l}\text { MILESTONE } \\
\text { LEVEL: }\end{array}$ & $\begin{array}{l}\text { MILESTONE } \\
\text { TYPE: }\end{array}$ & DNFSB STATUS: & DELIVERABLE: & \\
\hline $\begin{array}{l}\text { DOE-HQ } \\
\times \text { DOE-RL } \\
\text { DOE-FO } \\
\text { CONTRACTOR }\end{array}$ & $\begin{array}{l}\text { EA } \\
\text { PEG } \\
\times \text { OTHER } \\
\text { TIP }\end{array}$ & $\begin{array}{l}\text { DNFSB }(Y / N): N \\
\text { COMMIT \#: } \\
\text { RECOMM \#: }\end{array}$ & $\begin{array}{l}\text { Report } \\
\text { Letter } \\
\text { Drawing (s) } \\
\text { X Other (Specify) } \\
\text { SCHEDULE }\end{array}$ & $\begin{array}{l}X \text { DOE-RL } \\
\text { Other (Specify) }\end{array}$ \\
\hline
\end{tabular}

Description of what constitutes completion of this milestone:

Provide 00e-RL with a comprehensive schedule of current air and water permitting activities on the Hanford Site. 


\section{PHMC}

\section{MILESTONE DESCRIPTION SHEET}

\begin{tabular}{|c|c|c|c|c|}
\hline \multicolumn{4}{|c|}{$\begin{array}{l}\text { Title: PROVIDE RL WITH AIR/WATER PERMITIING } \\
\text { SCHEDULE (FOR FY 2000-2046) }\end{array}$} & Date: $8 / 25 / 98$ \\
\hline \multicolumn{4}{|c|}{ Assigned To: W.D. ADAIR } & CIN: \\
\hline \multicolumn{4}{|c|}{ Program WBS Designator: 1.8 .2 .3 .4} & Due Date: $5 / 03 / 00$ \\
\hline \multicolumn{5}{|c|}{ PBS No: RL-0T0I } \\
\hline \multicolumn{2}{|c|}{ MC \#: ECP-00-404 } & TPA No: & & Rev: 0 \\
\hline $\begin{array}{l}\text { MILESTONE } \\
\text { LEVEL: } \\
\text { DOE-HO } \\
\times \text { DOE-RL } \\
\text { DOE-FO } \\
\text { CONTRACTOR }\end{array}$ & $\begin{array}{l}\text { MILESTONE } \\
\text { TYPE: } \\
\\
\text { EA } \\
\text { PEG } \\
\text { X OTHER } \\
\text { TIP }\end{array}$ & $\begin{array}{l}\text { DNFSB STATUS: } \\
\text { DNFSB }(Y / N): N \\
\text { COMMIT \#: } \\
\text { RECOMM \#: }\end{array}$ & $\begin{array}{l}\text { DELIVERABLE: } \\
\text { Report } \\
\text { Letter } \\
\text { Orawing (s) } \\
\text { x Other (Specify) } \\
\text { SCHEDULE }\end{array}$ & $\begin{array}{l}\text { ADDRESS TO: } \\
\text { DOE-HQ } \\
\times \text { DOE-RL } \\
\text { Other (Specify) }\end{array}$ \\
\hline \multicolumn{5}{|c|}{$\begin{array}{l}\text { Milestone Description: } \\
\text { Provide DOE-RL with a schedule of air and water permitting activities. }\end{array}$} \\
\hline \multicolumn{5}{|c|}{$\begin{array}{l}\text { Description of what constitutes completion of this milestone: } \\
\text { Provide DOE-RL with a comprehensive schedule of current air and water } \\
\text { permitting activities on the Hanford Site. }\end{array}$} \\
\hline
\end{tabular}


HNF.SP-1112, Rev. 4

\section{PHMC}

MILESTONE DESCRIPTION SHEET

\begin{tabular}{|c|c|c|c|c|}
\hline \multicolumn{4}{|c|}{$\begin{array}{l}\text { TitTe: PROVIDE RL WITH AIR/WATER PERMITTING } \\
\text { SCHEDULE (FY 2000-2046) }\end{array}$} & Date: $8 / 25 / 99$ \\
\hline \multicolumn{4}{|c|}{ Assigned To: W.D. ADAIR } & CIN: \\
\hline \multicolumn{4}{|c|}{ Program WBS Designator: 1.8 .2 .3 .4} & Due Date: $7 / 01 / 00$ \\
\hline \multicolumn{5}{|c|}{ PBS No: RL-OT01 } \\
\hline \multicolumn{2}{|c|}{ MC \#: ECP-00-405 } & TPA No: & & Rev: 0 \\
\hline $\begin{array}{l}\text { MILESTONE } \\
\text { LEVEL: } \\
\\
\text { DOE-HQ } \\
\times \text { DOE-RL } \\
\text { DOEFO } \\
\text { CONTRACTOR }\end{array}$ & $\begin{array}{l}\text { MILESTONE } \\
\text { TYPE: } \\
\\
\text { EA } \\
\text { PEG } \\
\times \text { OTHER } \\
\text { TIP }\end{array}$ & $\begin{array}{l}\text { DNFSB STATUS: } \\
\text { DNFSB }(Y / N): N \\
\text { COMMIT \#: } \\
\text { RECOMM \#: }\end{array}$ & $\begin{array}{l}\text { DELIVERABLE: } \\
\text { Report } \\
\text { Letter } \\
\text { Drawing(s) } \\
\text { X Other (Specify) } \\
\text { SCHEDULE }\end{array}$ & $\begin{array}{l}\text { ADDRESS TO: } \\
\text { DOE-HQ } \\
\times \text { DOE-RL (Specify) } \\
\text { Other (Specis }\end{array}$ \\
\hline
\end{tabular}

Description of what constitutes completion of this milestone:

Provide DOE-RL with a comprhensive schedule of current air and water permitting activities on the Hanford Site. 
PHMC

MILESTONE DESCRIPTION SHEET

\begin{tabular}{|c|c|c|c|c|}
\hline \multicolumn{4}{|c|}{$\begin{array}{r}\text { Title: PROVIDE RL WITH AIR/WATER PERMITTIING } \\
\text { SCHEDULE (FOR FY 2000-2046) }\end{array}$} & Date: $8 / 25 / 98$ \\
\hline \multicolumn{4}{|c|}{ Assigned To: W.D. ADAIR } & CIN: \\
\hline \multicolumn{4}{|c|}{ Program WBS Designator: 1.8 .2 .3 .4} & Oue Date: 9/01/00 \\
\hline \multicolumn{5}{|c|}{ PBS No: RL-OT01 } \\
\hline \multicolumn{2}{|c|}{ MC \#: ECP-00-406 } & \multicolumn{2}{|l|}{ TPA No: } & Rev: 0 \\
\hline $\begin{array}{l}\text { MILESTONE } \\
\text { LEVEL: } \\
\text { DOE-HQ } \\
\times \text { DOE-RL } \\
\text { DOE-FO } \\
\text { CONTRACTOR }\end{array}$ & $\begin{array}{l}\text { MILESTONE } \\
\text { TYPE: } \\
\\
\text { EA } \\
\text { PEG } \\
\times \text { OTHER } \\
\text { TIP }\end{array}$ & $\begin{array}{l}\text { ONFSB STATUS: } \\
\text { DNFSB (Y/N): N } \\
\text { COMMIT \#: } \\
\text { RECOMM \#: }\end{array}$ & $\begin{array}{l}\text { DELIVERABLE: } \\
\\
\text { Report } \\
\text { Letter } \\
\text { Drawing(s) } \\
\text { X Other (Specify } \\
\text { SCHEDULE }\end{array}$ & $\begin{array}{l}\text { ADDRESS TO: } \\
\text { DOE-HQ } \\
\times \text { DOE-RL } \\
\text { Other (Specify) }\end{array}$ \\
\hline
\end{tabular}

Milestone Description:

Provide DOE-RL with a schedule of air and water permitting activities.

Description of what constitutes completion of this milestone:

Provide DOE-RL with a comprehensive schedule of current air and water permitting activities on the Hanford Site. 


\section{PHMC \\ MILESTONE DESCRIPTION SHEET}

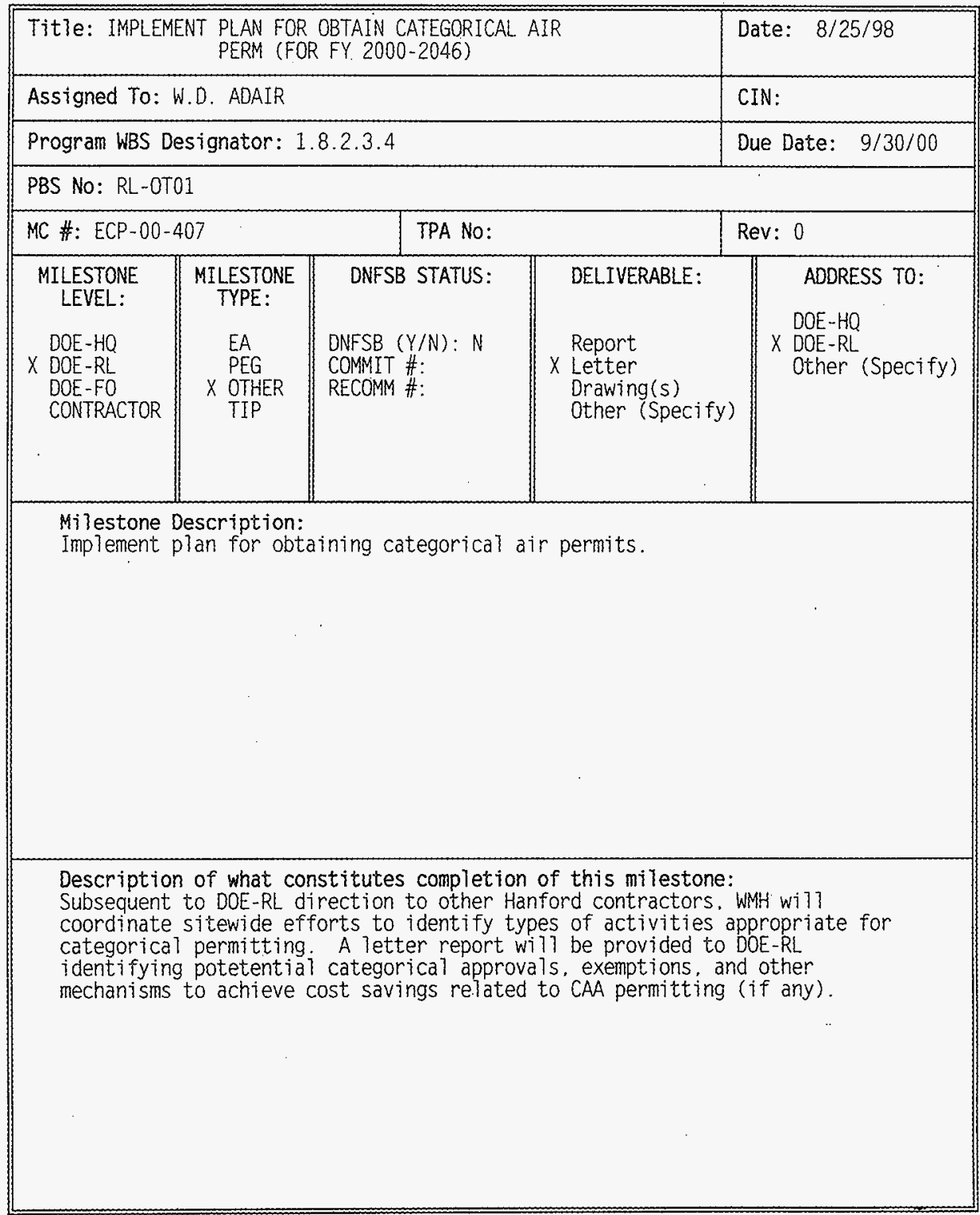




\section{PHMC}

\section{MILESTONE DESCRIPTION SHEET}

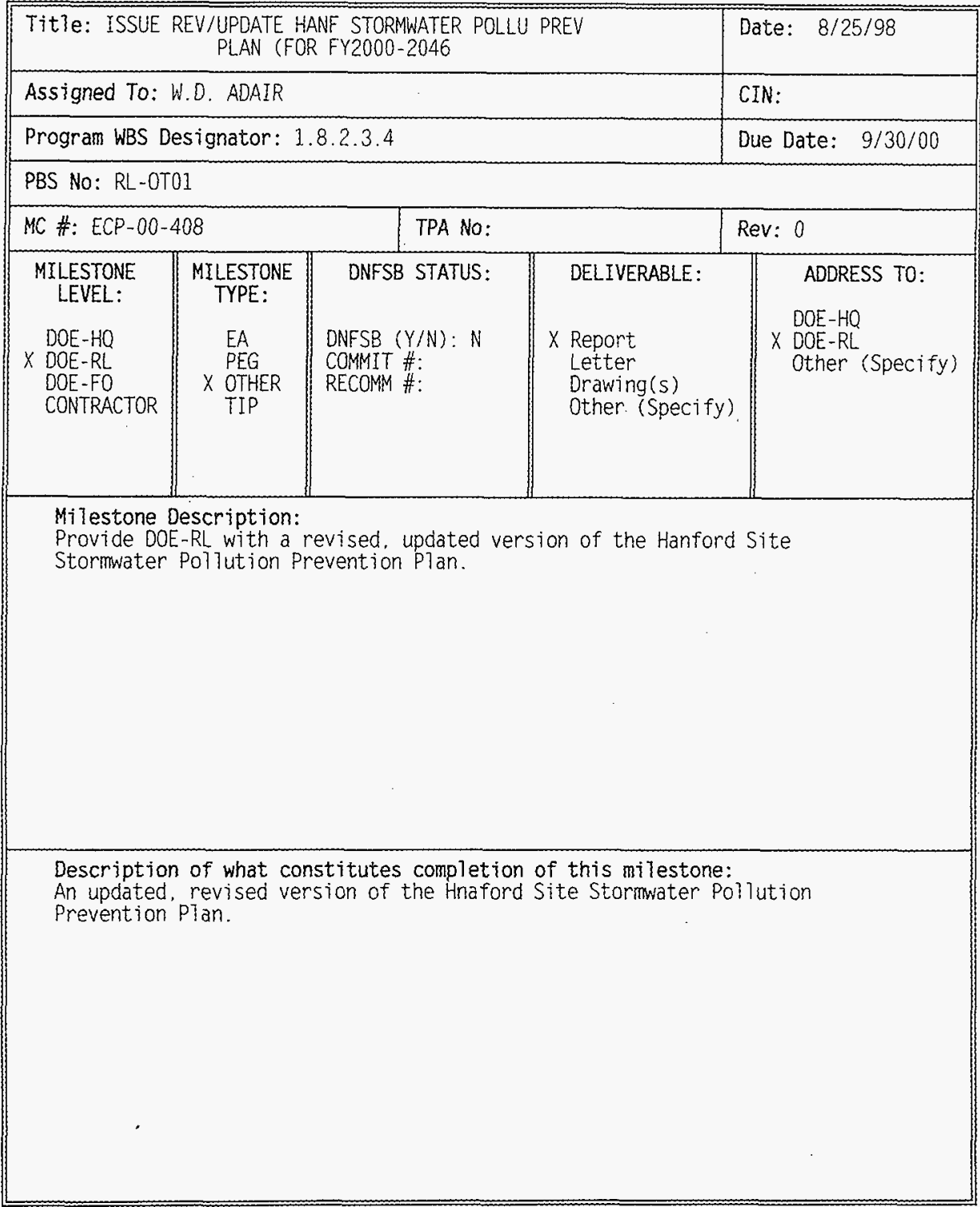


HNF-SP-1112, Rev. 4

\section{PHMC}

\section{MILESTONE DESCRIPTION SHEET}

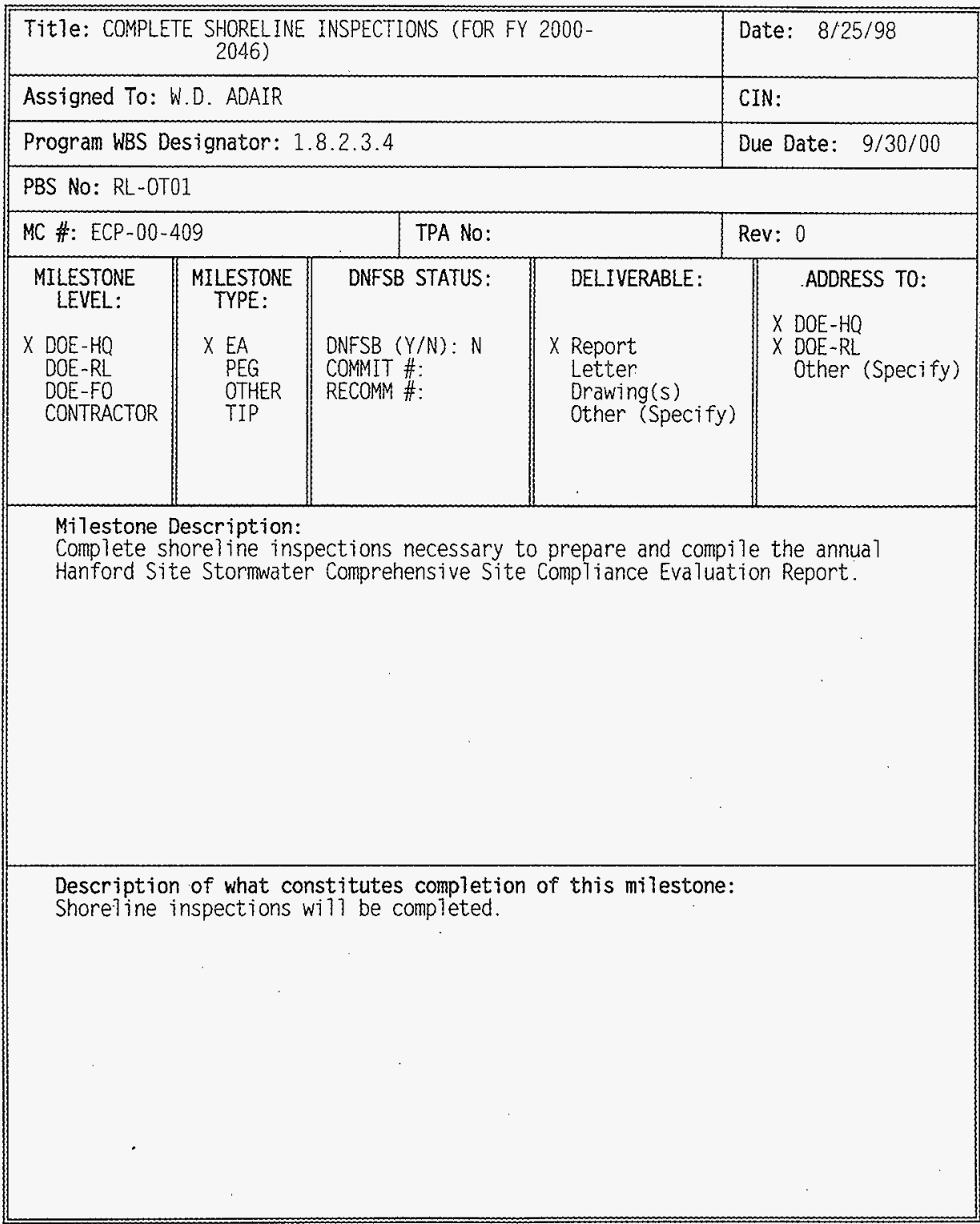


HNF-SP-1112, ReV. 4

\section{PHMC}

\section{MILESTONE DESCRIPTION SHEET}

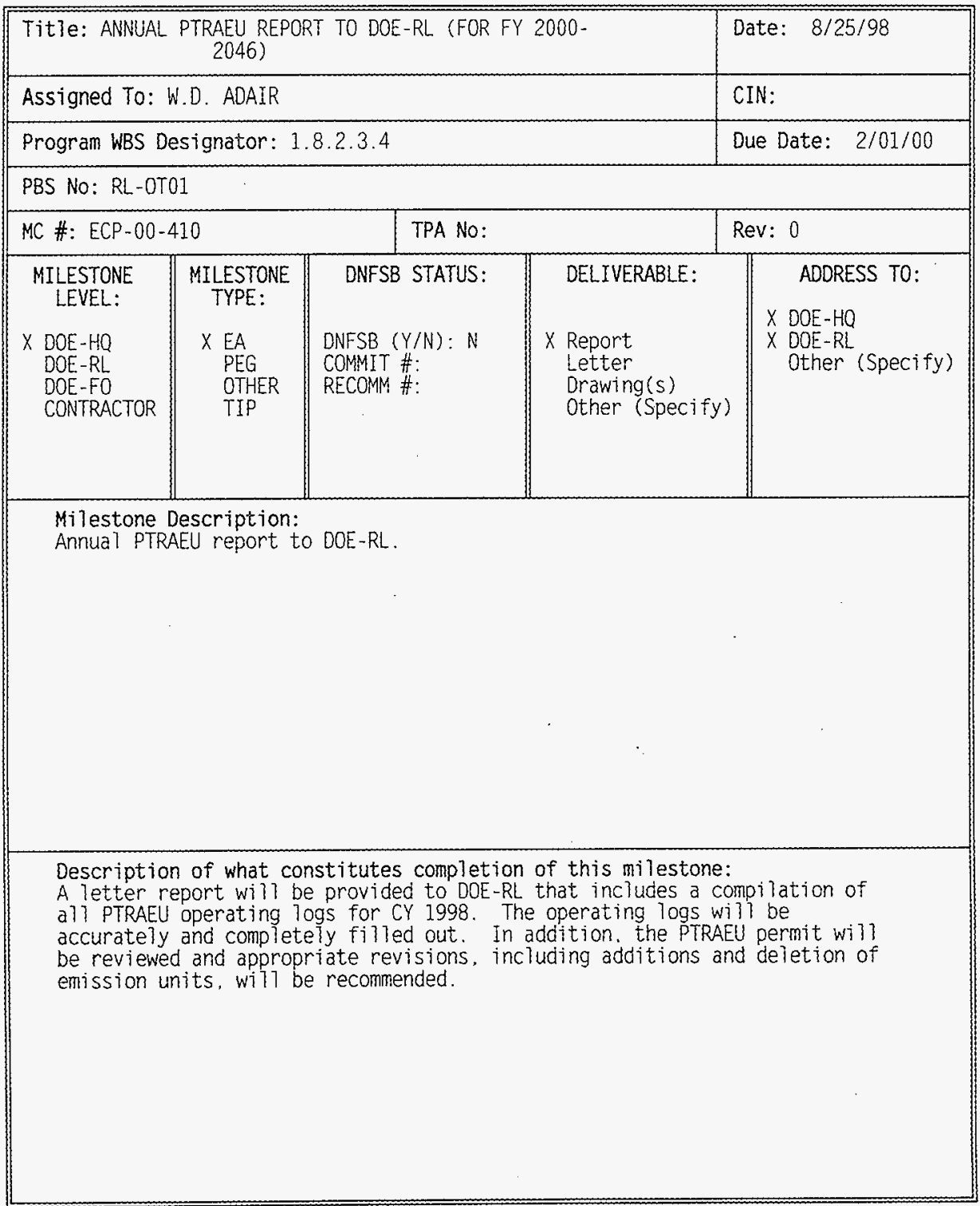


HNF-SP-1112, Rev. 4

PHMC

MILESTONE DESCRIPTION SHEET

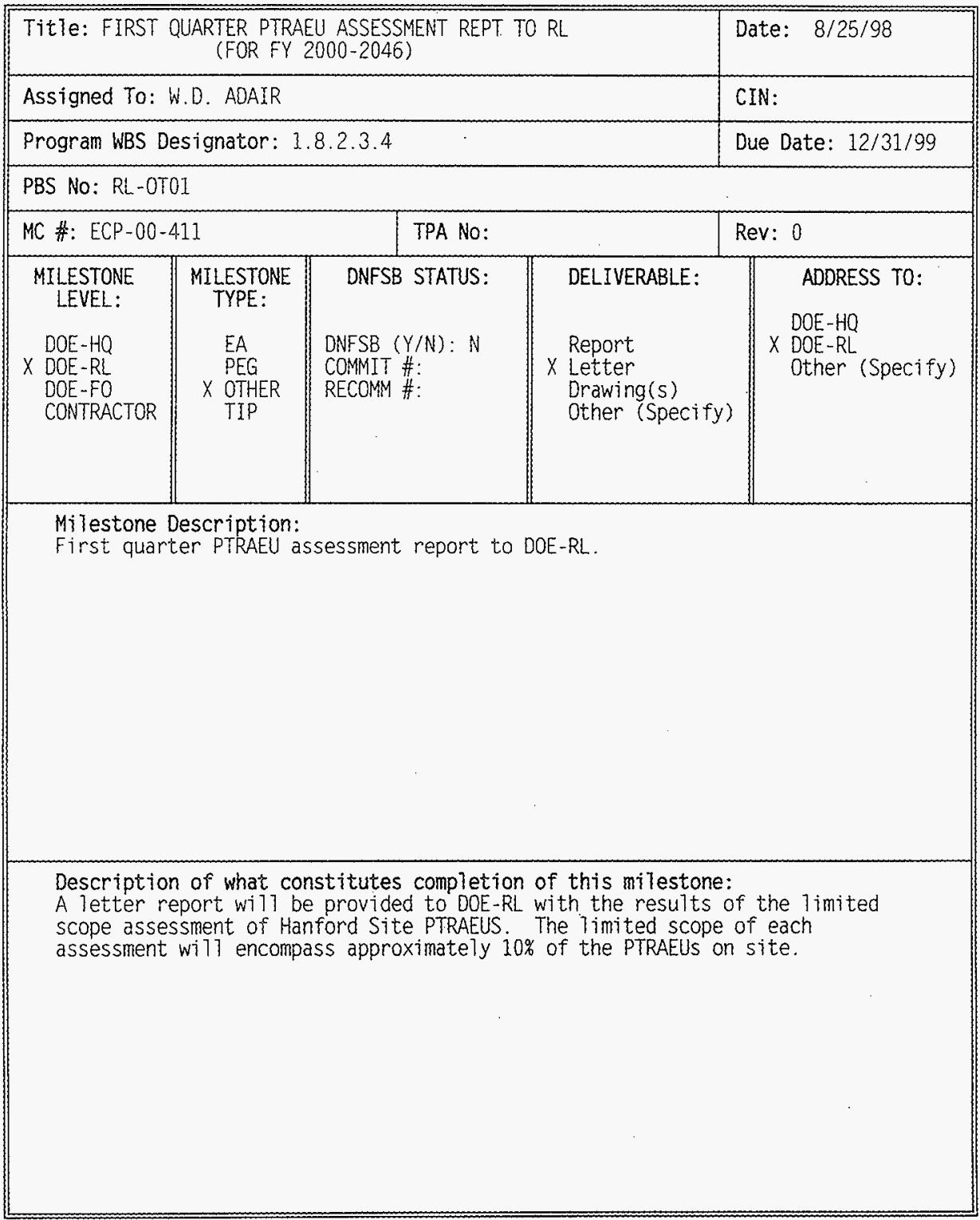


HNF-SP-1112, Rev. 4

\section{PHMC \\ MILESTONE DESCRIPTION SHEET}

\begin{tabular}{|c|c|c|c|c|}
\hline \multicolumn{4}{|c|}{$\begin{array}{c}\text { Title: SECOND QUARTER PTRAEU ASSESSMENT REPT TO RL } \\
\text { (FOR FY } 2000-2046)\end{array}$} & te: $8 / 25 / 98$ \\
\hline \multicolumn{4}{|c|}{ Assigned To: W.D. ADAIR } & CIN: \\
\hline \multicolumn{4}{|c|}{ Program WBS Designator: $1.8 \cdot 2.3 .4$} & e Date: $3 / 31 / 00$ \\
\hline \multicolumn{5}{|c|}{ PBS No: RL-OT01 } \\
\hline \multicolumn{2}{|c|}{ MC \#: ECP-00-412 } & TPA No: & & Rev: 0 \\
\hline $\begin{array}{l}\text { MILESTONE } \\
\text { LEVEL: } \\
\text { DOE-HQ } \\
\times \text { DOE-RL } \\
\text { DOE-FO } \\
\text { CONTRACTOR }\end{array}$ & $\begin{array}{l}\text { MILESTONE } \\
\text { TYPE: } \\
\\
\text { EA } \\
\text { PEG } \\
\times \text { OTHER } \\
\text { TIP }\end{array}$ & $\begin{array}{l}\text { DNFSB STATUS: } \\
\text { ONFSB }(Y / N): N \\
\text { COMMIT \#: } \\
\text { RECOMM \#: }\end{array}$ & $\begin{array}{l}\text { DELIVERABLE: } \\
\text { Report } \\
\times \text { Letter } \\
\text { Drawing(s) } \\
\text { Other (Specify) }\end{array}$ & $\begin{array}{l}\text { ADDRESS TO: } \\
\text { DOE-HO } \\
\times \text { DOE-RL } \\
\text { Other (Specify) }\end{array}$ \\
\hline \multicolumn{5}{|c|}{$\begin{array}{l}\text { Milestone Description: } \\
\text { Second quarter PTRAEU assessment report to DOE-RL. }\end{array}$} \\
\hline \multicolumn{5}{|c|}{$\begin{array}{l}\text { Description of what constitutes completion of this milestone: } \\
\text { A letter report will be provided to DOE-RL with the results of a limited } \\
\text { scope assessment of Hanford Site PTRAEUS. The limited scope of each } \\
\text { assessment will encompass approximately 10\% of the PTRAEUS on site. }\end{array}$} \\
\hline
\end{tabular}


HNF-SP-1112, Rev. 4

PHMC

MILESTONE DESCRIPTION SHEET

\begin{tabular}{|c|c|c|c|c|}
\hline \multicolumn{4}{|c|}{$\begin{array}{c}\text { Title: THIRD QUARTER PTRAEU ASSESSMENT REPT TO RL } \\
\text { (FOR FY } 2000-2046)\end{array}$} & Date: $8 / 25 / 98$ \\
\hline \multicolumn{4}{|c|}{ Assigned To: W.D. ADAIR } & CIN: \\
\hline \multicolumn{4}{|c|}{ Program WBS Designator: 1.8 .2 .3 .4} & Due Date: $6 / 30 / 00$ \\
\hline \multicolumn{5}{|c|}{ PBS NO: RL-OTO1 } \\
\hline \multicolumn{2}{|c|}{ MC \#: ECP-00-413 } & \multicolumn{2}{|l|}{ TPA NO: } & Rev: 0 \\
\hline $\begin{array}{l}\text { MILESTONE } \\
\text { LEVEL: }\end{array}$ & $\begin{array}{l}\text { MILESTONE } \\
\text { TYPE: }\end{array}$ & DNFSB STATUS: & DELIVERABLE: & \\
\hline $\begin{array}{l}\text { DOE-HQ } \\
\times \text { DOE-RL } \\
\text { DOE-FO } \\
\text { CONTRACTOR }\end{array}$ & $\begin{array}{l}\text { EA } \\
\text { PEG } \\
\times \text { OTHER } \\
\text { TIP }\end{array}$ & $\begin{array}{l}\text { DNFSB }(Y / N): N \\
\text { COMMIT \#: } \\
\text { RECOMM \#: }\end{array}$ & $\begin{array}{l}\text { Report } \\
X \text { Letter } \\
\text { Drawing (s) } \\
\text { Other (Specify) }\end{array}$ & $\begin{array}{l}\times \text { DOE- }-\mathrm{RL} \\
\text { Other (Specify) }\end{array}$ \\
\hline
\end{tabular}

Milestone Description:

Third quarter PTRAEU assessment report to DOE-RL.

Description of what constitutes completion of this milestone:

A letter report will be provided to DOE-RL with the results of a limited scope assessment of Hanford Site PTRAEUS. The limited scope of each assessment wi11 encompass approximately $10 \%$ of the PTRAEUS on site. 
HNF-SP-1112, Rev. 4

PHMC

MILESTONE DESCRIPTION SHEET

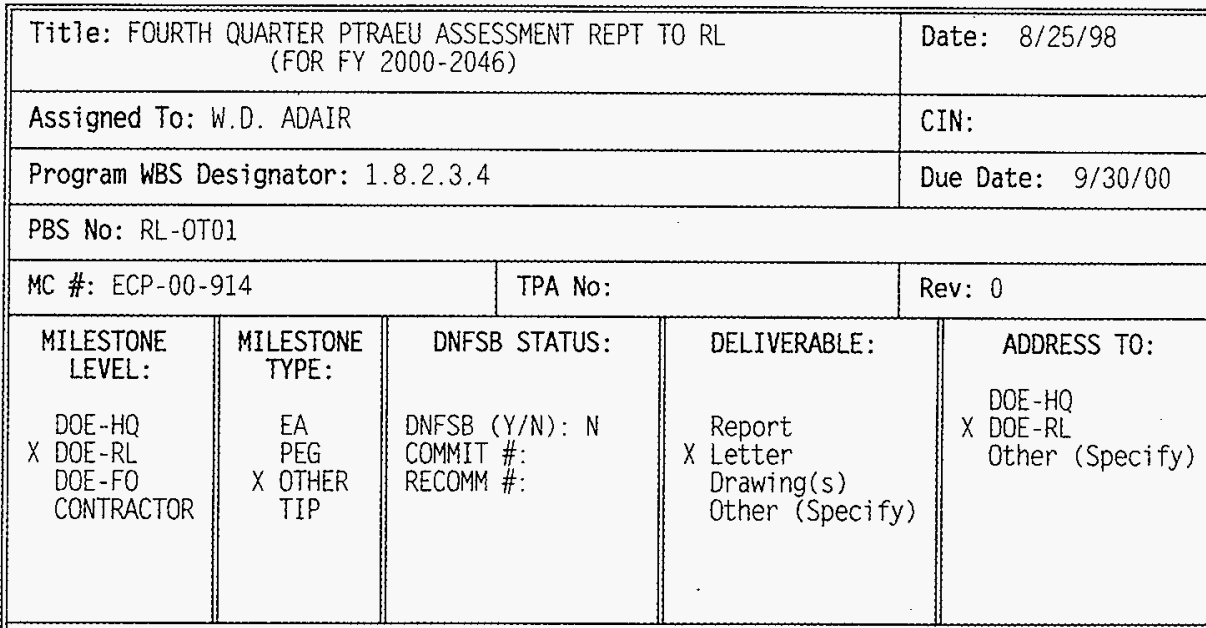

Milestone Description:

Fourth quarter PTRAEU assessment report to DOE-RL.

Description of what constitutes completion of this milestone:

A letter report will be provided to OOE-RL with the results of a limited scope assessment wi 71 encompass approximately $10 \%$ of the PTRAEUS on site. 
PHMC

MILESTONE DESCRIPTION SHEET

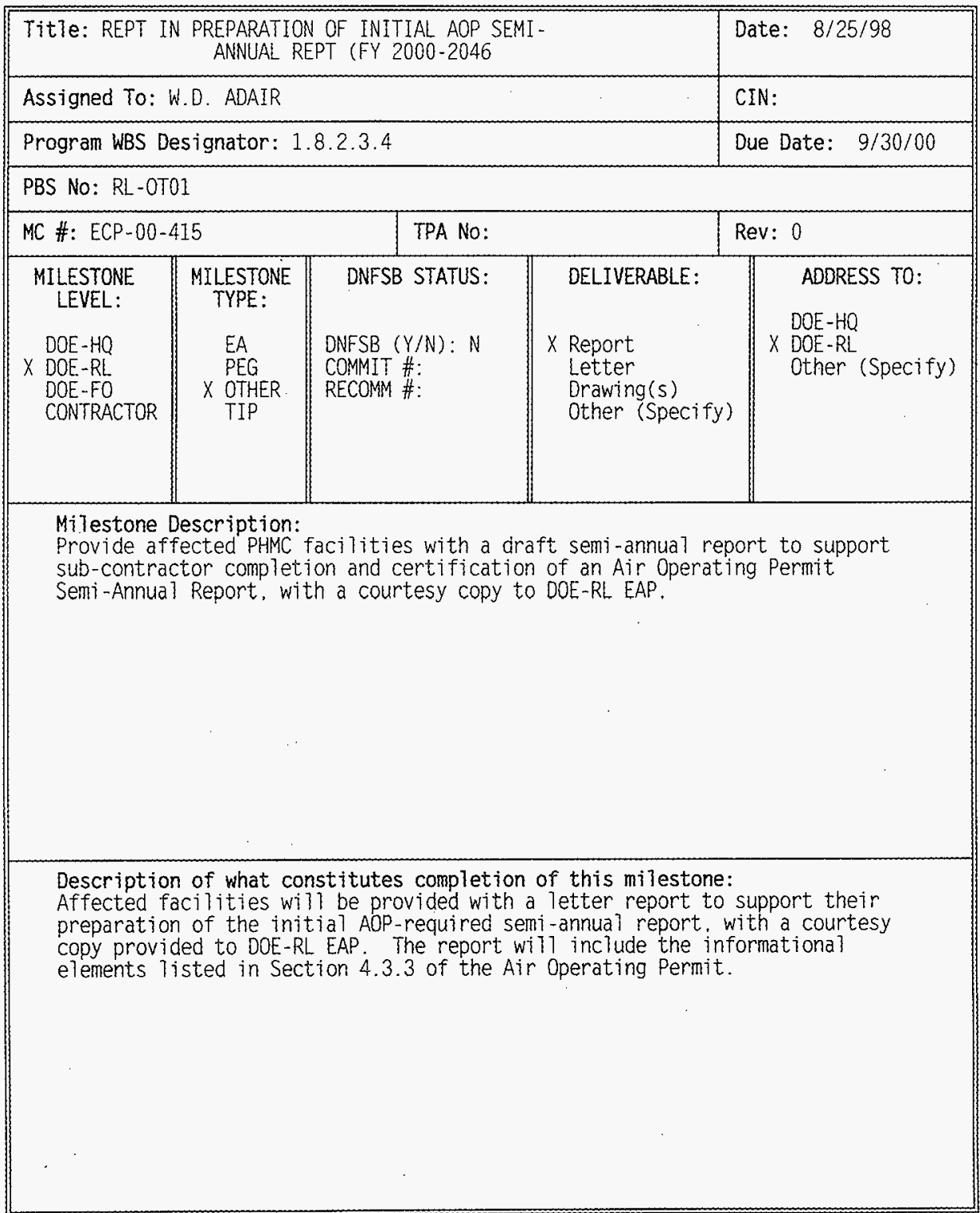




\section{PHMC}

\section{MILESTONE DESCRIPTION SHEET}

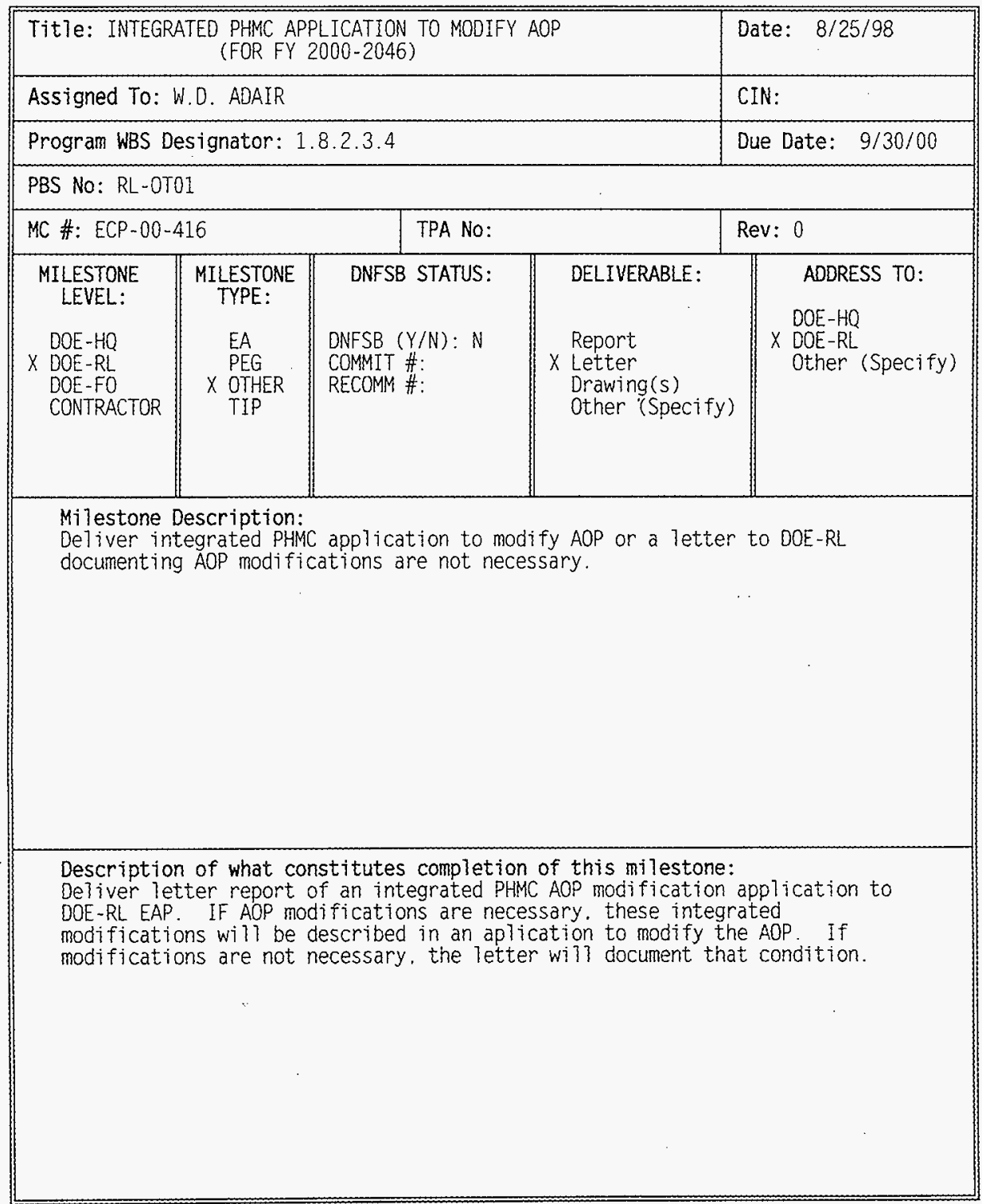


HNF-SP-1112, Rev. 4

PHMC

MILESTONE DESCRIPTION SHEET

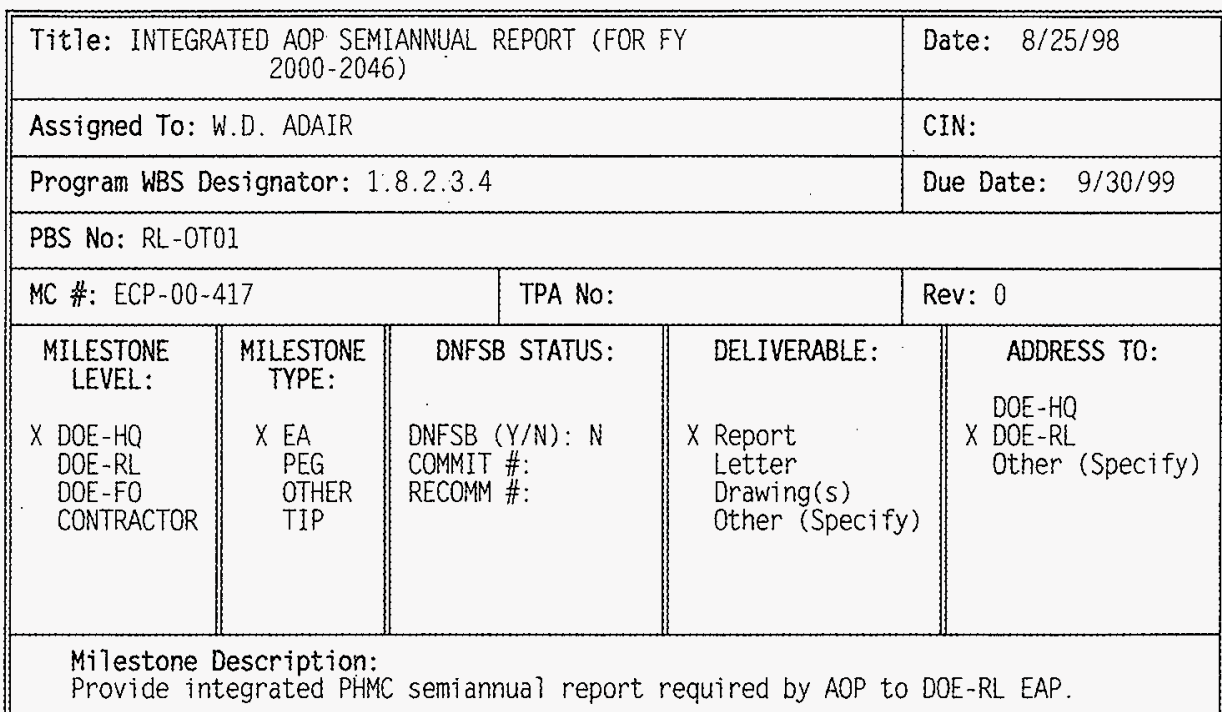

Description of what constitutes completion of this milestone:

Provide DOE-RL with a semiannual report, certified by FDH as true, accurate, and complete. to support transmittal to the regulatory agencies. to satisfy the terms and conditions of the AOP. 
HNF-SP-1112, Rev. 4

\section{PHMC \\ MILESTONE DESCRIPTION SHEET}

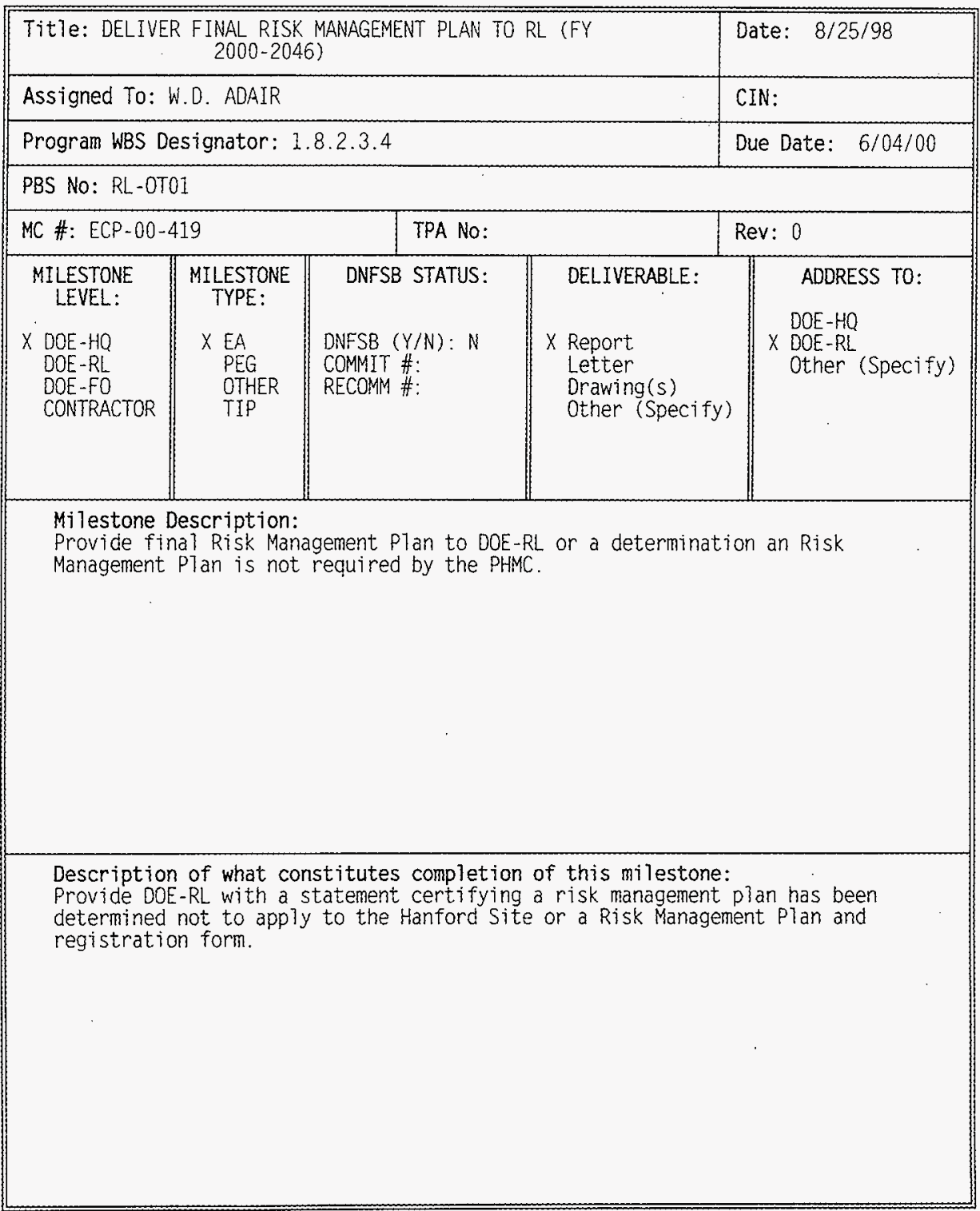




\section{PHMC}

MILESTONE DESCRIPTION SHEET

\begin{tabular}{|c|c|c|c|c|}
\hline \multicolumn{4}{|c|}{$\begin{aligned} \text { Title: EPCRA } 312 & \text { TIER TWO EMERG \& HAZ CHEM } \\
& \text { INVENTORY REPT (FY 2000-2046) }\end{aligned}$} & Date: $8 / 25 / 98$ \\
\hline \multicolumn{4}{|c|}{ Assigned To: WD ADAIR } & CIN: \\
\hline \multicolumn{4}{|c|}{ Program WBS Designator: 1.8 .2 .3 .5} & Due Date: 2/22/00 \\
\hline \multicolumn{5}{|c|}{ PBS No: RL-0T01 } \\
\hline \multicolumn{2}{|c|}{ MC \#: ECP-00-501 } & TPA No: & & Rev: 0 \\
\hline $\begin{array}{l}\text { MILESTONE } \\
\text { LEVEL: } \\
\text { X DOE-HQ } \\
\text { DOE-RL } \\
\text { DOE-FO } \\
\text { CONTRACTOR }\end{array}$ & $\begin{array}{l}\text { MILESTONE } \\
\text { TYPE: } \\
\text { X EA } \\
\text { PEG } \\
\text { OTHER } \\
\text { TIP }\end{array}$ & $\begin{array}{l}\text { DNFSB STATUS: } \\
\text { DNFSB (Y/N): N } \\
\text { COMMIT \#: } \\
\text { RECOMM \#: }\end{array}$ & $\begin{array}{l}\text { DELIVERABLE: } \\
\text { Report } \\
\text { Letter } \\
\text { X Drawing(s) } \\
\text { X Other (Specify) } \\
\text { FORMS }\end{array}$ & $\begin{array}{l}\text { ADDRESS TO: } \\
\text { DOE-HO } \\
\times \text { DOE-RL } \\
\text { Other (Specify) }\end{array}$ \\
\hline \multicolumn{5}{|c|}{$\begin{array}{l}\text { MiTestone Description: } \\
\text { The Tier Two Emergency and Hazardous Chemical Inventory (Tier Two) report } \\
\text { contains hazards, storage, and location information on Hazardous chemicals } \\
\text { stored in the Hanford Site in amounts exceeding minimum threshold levels } \\
\text { established in } 40 \text { CFR } 370.20 \text {. The requirements are identified in } 40 \text { CFR } \\
370.25 \text { and report format identified } 40 \text { CFR } 370.41 \text {. Data received from } \\
\text { PHMC-managed projects/facilities will be certified. Other site contractors } \\
\text { will be requested to provide certification for data submitted for their } \\
\text { respective projects/facilities. }\end{array}$} \\
\hline \multicolumn{5}{|c|}{$\begin{array}{l}\text { Description of what constitutes completion of this miTestone: } \\
\text { Submit Tier Two Report to DOE-RL by February 22. } 2000 \text {, and coordinate } \\
\text { preparation and review of the Tier Two report to a } 71 \text { w transmittal by DOE- } \\
\text { RL to the Washington State Emergency Response Commission. local energency } \\
\text { planning committees, and local fire departments by the March 1. 2000. } \\
\text { regulatory due date. }\end{array}$} \\
\hline
\end{tabular}




\section{PHMC}

\section{MILESTONE DESCRIPTION SHEET}

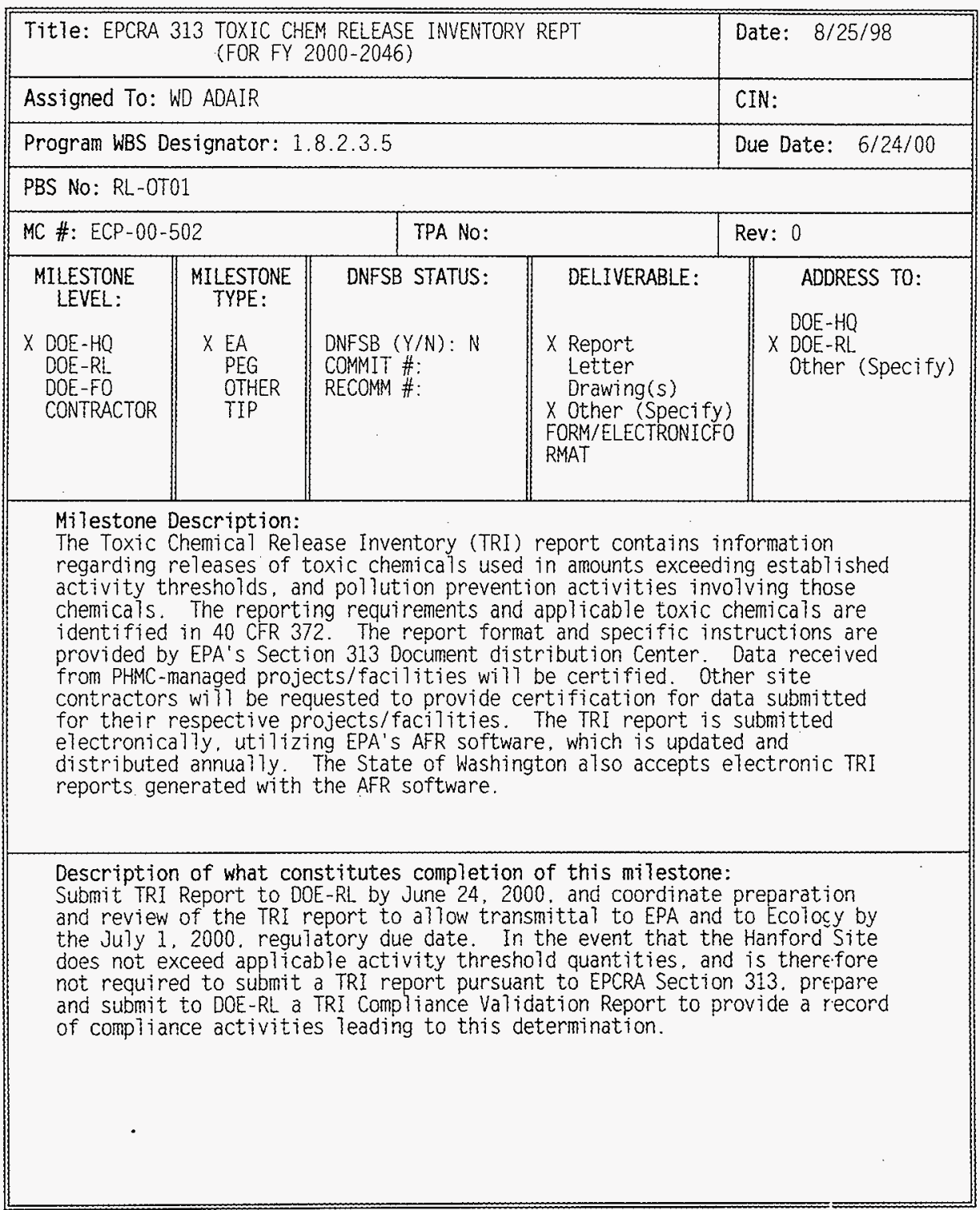




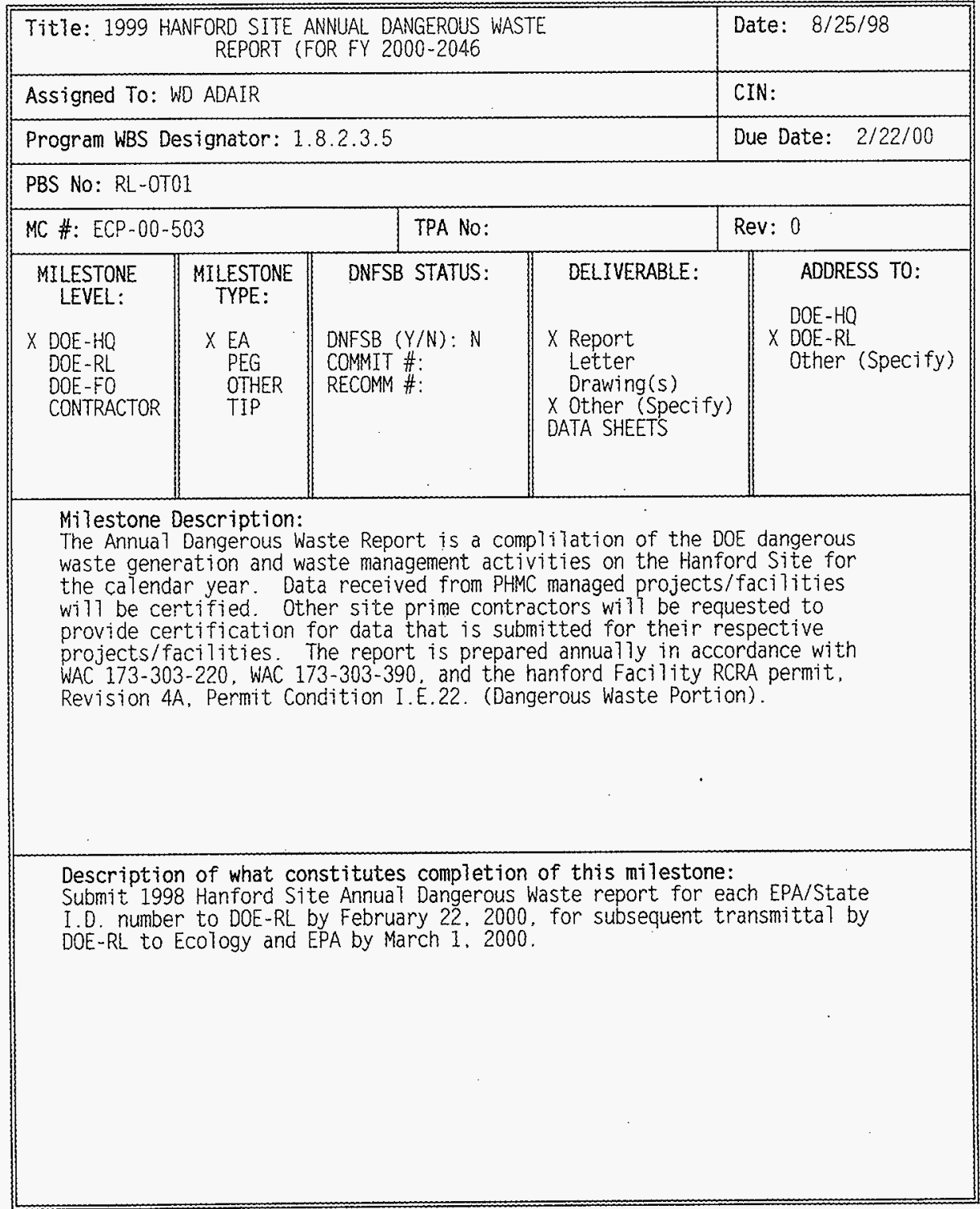


HNF-SP-1112, Rev. 4

\section{PHMC}

\section{MILESTONE DESCRIPTION SHEET}

\begin{tabular}{|c|c|c|c|c|}
\hline \multicolumn{4}{|c|}{$\begin{array}{c}\text { Title: } 1999 \text { HANFORD SITE ANNUAL PCB OOCUMENT LOG } \\
\text { (FOR FY 2000-2046) }\end{array}$} & Date: $8 / 25 / 98$ \\
\hline \multicolumn{4}{|c|}{ Assigned To: WD ADAIR } & CIN: \\
\hline \multicolumn{4}{|c|}{ Program WBS Designator: 1.8.2.3.5 } & Due Date: $6 / 24 / 00$ \\
\hline \multicolumn{5}{|c|}{ PBS No: RL-OT01 } \\
\hline \multicolumn{2}{|c|}{ MC \#: ECP-00-504 } & \multicolumn{2}{|l|}{ TPA No: } & Rev: 0 \\
\hline $\begin{array}{l}\text { MILESTONE } \\
\text { LEVEL: }\end{array}$ & $\begin{array}{l}\text { MILESTONE } \\
\text { TYPE: }\end{array}$ & DNFSB STATUS: & DELIVERABLE: & ADDRESS TO: \\
\hline $\begin{array}{l}X \text { DOE-HQ } \\
\text { DOE-RL } \\
\text { DOE-FO } \\
\text { CONTRACTOR }\end{array}$ & $\begin{array}{l}\times \text { EA } \\
\text { PEG } \\
\text { OTHER } \\
\text { TIP }\end{array}$ & $\begin{array}{l}\text { DNFSB }(Y / N): N \\
\text { COMMIT \#: } \\
\text { RECOMMI \#: }\end{array}$ & $\begin{array}{l}\text { Report } \\
\times \text { Letter } \\
\text { Drawing(s) } \\
x \text { Other (Specify) } \\
\text { DATA SHEETS }\end{array}$ & $\begin{array}{l}\text { DOE-HQ } \\
\times \text { DOE-RL } \\
\text { Other (Specify) }\end{array}$ \\
\hline
\end{tabular}

Milestone Description:

Prepare a PCB annual document log that provides information on a 17 DOE PCB waste handling activities on the Hanford site regulated under TSCA. The detajls for the log content and preparation time frame are contained in 40 CFR 761.180

Description of what constitutes completion of this milestone:

Submit the 1998 Hanford Site Annual PCB Document Log to DOE-RL by June 24. 2000 . 
HNF-SP-1112, Rev. 4

PHMC

MILESTONE DESCRIPTION SHEET

\begin{tabular}{|c|c|c|c|c|}
\hline \multicolumn{4}{|c|}{ Title: 1999 ANNUAL PCB REPORT (FOR FY 2000-2046) } & Date: $8 / 25 / 98$ \\
\hline \multicolumn{4}{|c|}{ Assigned To: WD ADAIR } & CIN: \\
\hline \multicolumn{4}{|c|}{ Program WBS Designator: 1.8 .2 .3 .5} & Due Date: $7 / 08 / 00$ \\
\hline \multicolumn{5}{|c|}{ PBS NO: RL-OT01 } \\
\hline \multicolumn{2}{|c|}{ MC \#: ECP-00-505 } & TPA NO: & & Rev: 0 \\
\hline $\begin{array}{l}\text { MILESTONE } \\
\text { LEVEL: } \\
\times \text { DOE-HQ } \\
\text { DOE-RL } \\
\text { DOE-FO } \\
\text { CONTRACTOR }\end{array}$ & $\begin{array}{l}\text { MILESTONE } \\
\text { TYPE: } \\
\text { X EA } \\
\text { PEG } \\
\text { OTHER } \\
\text { TIP }\end{array}$ & $\begin{array}{l}\text { DNFSB STATUS: } \\
\text { DNFSB }(Y / N): N \\
\text { COMMIT \#: } \\
\text { RECOMM \#: }\end{array}$ & $\begin{array}{l}\text { DELIVERABLE: } \\
x \text { Report } \\
X \text { Letter } \\
\text { Drawing(S) } \\
x \text { Other (Specify) } \\
\text { DATA SHEETS }\end{array}$ & $\begin{array}{l}\text { ADDRESS TO: } \\
\text { DOE-HO } \\
\times \text { DOE-RL } \\
\text { Other (Specify) }\end{array}$ \\
\hline \multicolumn{5}{|c|}{$\begin{array}{l}\text { Milestone Description: } \\
\text { The } 1999 \text { Annual PCB report provides summary information on the PCB waste } \\
\text { handling activities for Trench } 94 \text { of the } 218-\mathrm{E}-12 \mathrm{~B} \text { Burial Ground in the } 200 \\
\text { East Area and any other PCB disposal units on the Hanford Site regulated } \\
\text { under TSCA. The report is prepared annually as required by } 40 \text { CFR } 761.180 \text {. }\end{array}$} \\
\hline \multicolumn{5}{|c|}{$\begin{array}{l}\text { Description of what constitutes completion of this milestone: } \\
\text { Submit the } 1999 \text { Annual PCB report to D0E-RL by July } 8,2000 \text {, for subsequent } \\
\text { transmittal by DOE-RL to EPA by July } 15,2000 \text {. }\end{array}$} \\
\hline
\end{tabular}


PHMC

MILESTONE DESCRIPTION SHEET

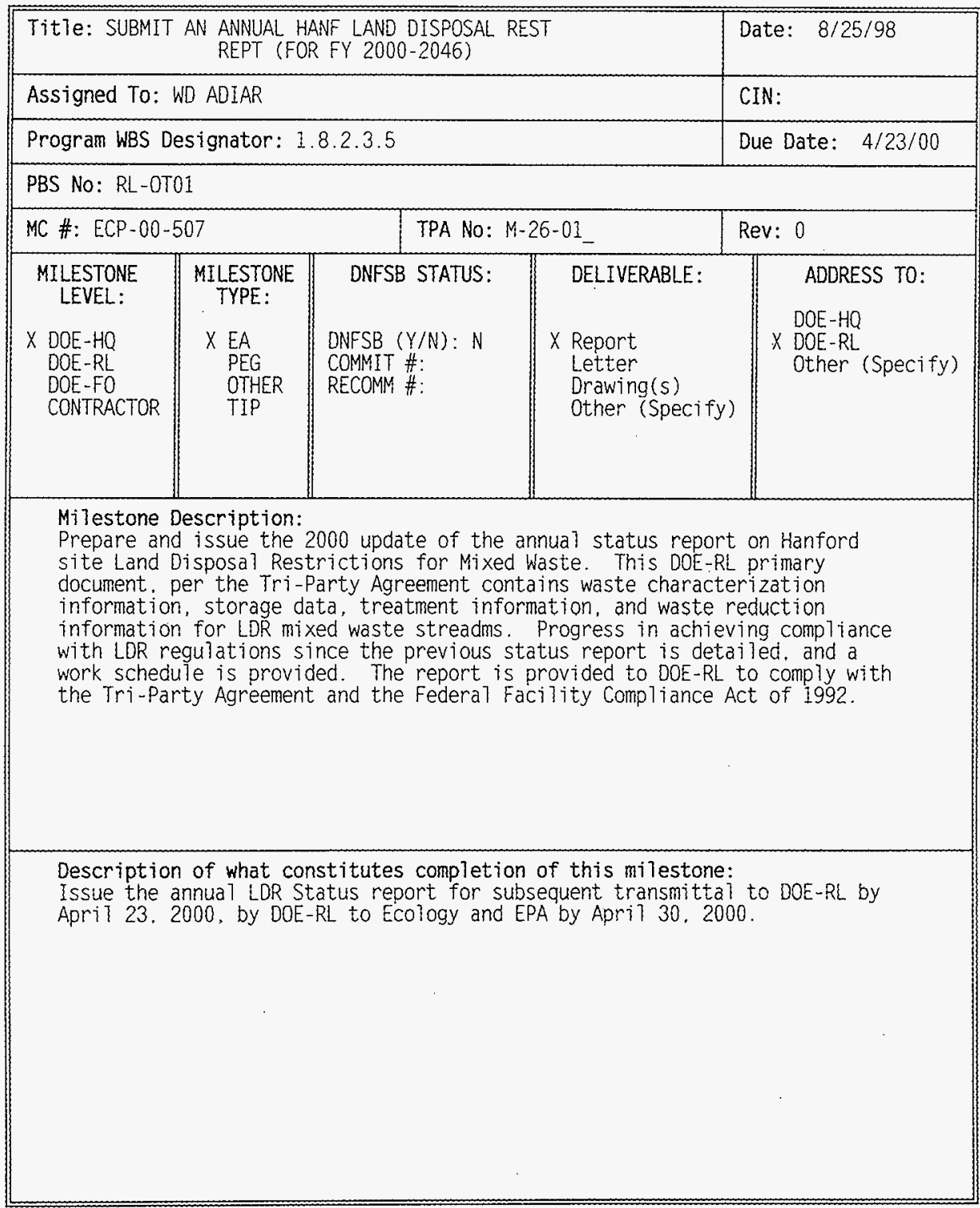




\section{PHMC}

\section{MILESTONE DESCRIPTION SHEET}

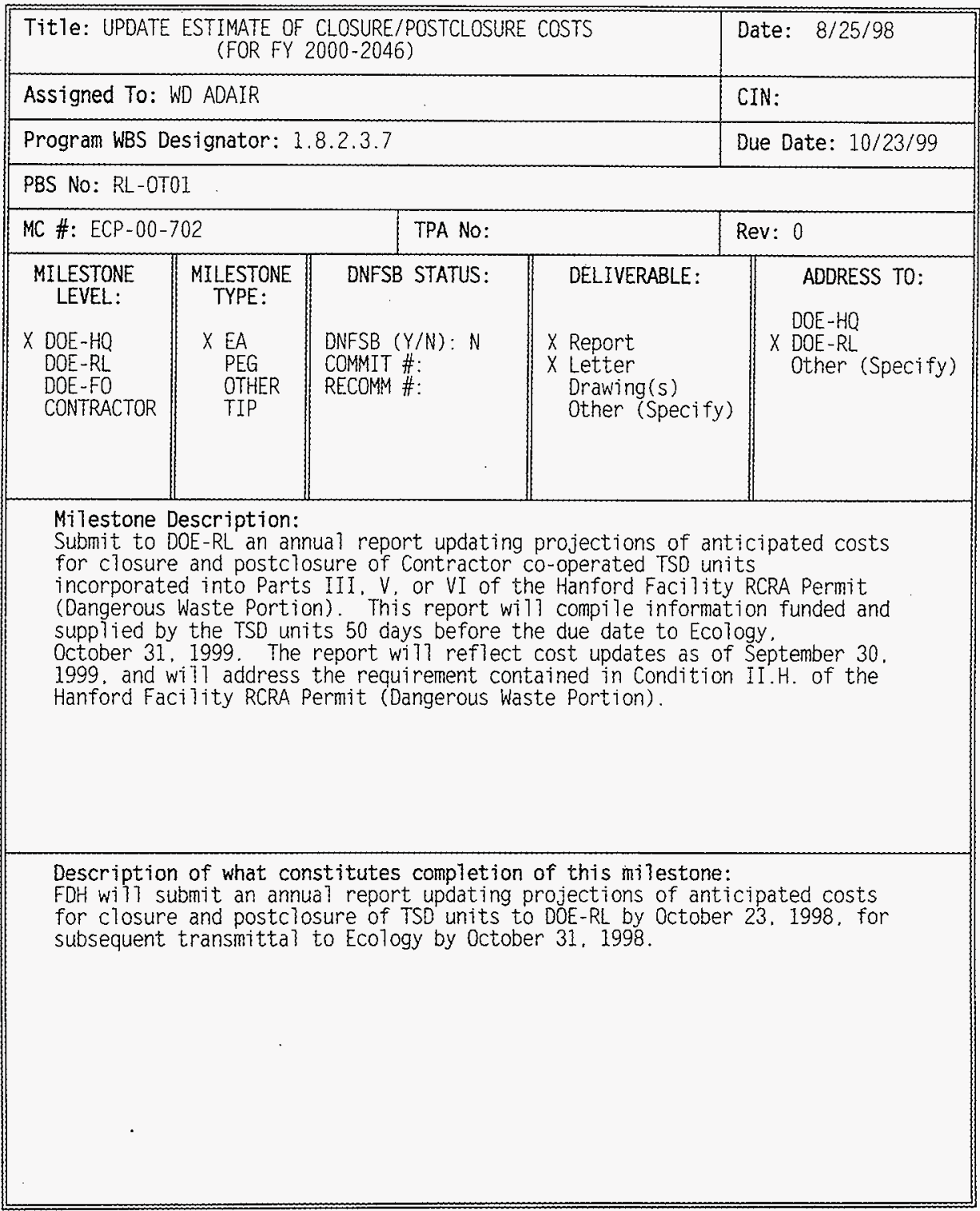




\section{PHMC}

MILESTONE DESCRIPTION SHEET

\begin{tabular}{|c|c|c|c|c|}
\hline \multicolumn{4}{|c|}{$\begin{array}{c}\text { Title: COORDINATE RCRA PIPE MAPPING AND MARKING } \\
\text { (FOR FY 2000-2046) }\end{array}$} & ate: $8 / 25 / 98$ \\
\hline \multicolumn{4}{|c|}{ Assigned To: WD ADAIR } & CIN: \\
\hline \multicolumn{4}{|c|}{ Program WBS Designator: $1.8 \cdot 2.3 .7$} & Due Date: $9 / 21 / 00$ \\
\hline \multicolumn{5}{|c|}{ PBS No: RL-0T01 } \\
\hline \multicolumn{2}{|c|}{ MC \#: ECP-00-703 } & TPA No: & & Rev: 0 \\
\hline $\begin{array}{l}\text { MILESTONE } \\
\text { LEVEL: } \\
\text { X DOE-HO } \\
\text { DOE-RL } \\
\text { DOE-FO } \\
\text { CONTRACTOR }\end{array}$ & $\begin{array}{l}\text { MILESTONE } \\
\text { TYPE: } \\
\times \text { EA } \\
\text { PEG } \\
\text { OTHER } \\
\text { TIP }\end{array}$ & $\begin{array}{l}\text { DNFSB STATUS: } \\
\text { ONFSB }(Y / N): N \\
\text { COMMIT \#: } \\
\text { RECOMM \#: }\end{array}$ & $\begin{array}{l}\text { DELIVERABLE: } \\
\text { Report } \\
\text { Letter } \\
\text { Drawing(s) } \\
\text { x Other (Specify) }\end{array}$ & $\begin{array}{l}\text { ADDRESS TO: } \\
\text { DOE-HQ } \\
\times \text { DOE-RL } \\
\text { Other (Specify) }\end{array}$ \\
\hline \multicolumn{5}{|c|}{$\begin{array}{l}\text { Milestone Description: } \\
\text { By September } 21 \text {. 2000 deliver to DOE-RL a report updating the Hanford } \\
\text { Facility RCRA Permit Mapping Information Report as required by Permit } \\
\text { Conditions II. U and II.V. The mapping report will contain/be based on } \\
\text { information received in complete form from Contractor co-operated TSO units } \\
\text { six months before the due date to Ecology of September } 28 \text {, } 2000 \text {. If the } \\
\text { report does not require updating, a letter to DOE-RL confirming this fact } \\
\text { will be delivered by September } 21.2000 \text {. }\end{array}$} \\
\hline \multicolumn{5}{|c|}{$\begin{array}{l}\text { Description of what constitutes completion of this milestone: } \\
\text { By September } 21 \text {, } 2000 \text {, submit to DOE-RL the Hanford Facility RCRA updates } \\
\text { to the permit Mapping Information Report for Condition II.U. for } \\
\text { subsequent transmittal by DOE-RL to Ecology by September } 28,2000 \text {. }\end{array}$} \\
\hline
\end{tabular}


HNF-SP-1112, Rev. 4

\section{PHMC}

\section{MILESTONE DESCRIPTION SHEET}

\begin{tabular}{|c|c|c|c|c|}
\hline \multicolumn{4}{|c|}{$\begin{array}{c}\text { Title: TRANSMIT EIS/ODIS DATA TO INEEL (FOR FY } 2000 \\
-2046)\end{array}$} & Date: $8 / 25 / 98$ \\
\hline \multicolumn{4}{|c|}{ Assigned To: WD ADAIR } & CIN: \\
\hline \multicolumn{4}{|c|}{ Program WBS Designator: $1.8 \cdot 2.3 .8$} & Due Date: $4 / 01 / 00$ \\
\hline \multicolumn{5}{|c|}{ PBS No: RL-0T01 } \\
\hline \multicolumn{2}{|c|}{ MC \#: ECP-00-801 } & \multicolumn{2}{|l|}{ TPA No: } & Rev: 0 \\
\hline $\begin{array}{l}\text { MILESTONE } \\
\text { LEVEL: } \\
\times \text { DOE-HO } \\
\text { DOE-RL } \\
\text { DOE-FO } \\
\text { CONTRACTOR }\end{array}$ & $\begin{array}{l}\text { MILESTONE } \\
\text { TYPE: } \\
\times \text { EA } \\
\text { PEG } \\
\text { OTHER } \\
\text { TIP }\end{array}$ & $\begin{array}{l}\text { DNFSB STATUS: } \\
\text { DNFSB }(Y / N): N \\
\text { COMMIT \#: } \\
\text { RECOMM \#: }\end{array}$ & $\begin{array}{l}\text { DELIVERABLE: } \\
\text { Report } \\
\text { Letter } \\
\text { Drawing(s) } \\
\text { X Other (Specify) } \\
\text { ELECTRONIC DATA }\end{array}$ & $\begin{array}{l}\text { ADDRESS TO: } \\
\text { DOE-HQ } \\
\text { DOE-RL } \\
\times \text { Other (SPECify) } \\
\text { IDAHO NATIONAL } \\
\text { ENG LAB (DOE) }\end{array}$ \\
\hline \multicolumn{5}{|c|}{$\begin{array}{l}\text { Milestone Description: } \\
\text { DOE requires its sites to annually compile and send radionuclide release } \\
\text { data, for both } 3 \text { iquid and airborne discharges, to the Idaho National } \\
\text { Environmental Engineering Laboratory (INEE() in Idaho Falls. Idaho, in } \\
\text { accordance with DOE Order } 5400.1 \text {. This data-collecting program is called } \\
\text { the Effluent Information system - Onsite Discharge Information System } \\
\text { (EIS/ODIS). NOTE: Early transmission to DOE-RL is not required. The } \\
\text { electronic format is not reviewed by DOE-RL. }\end{array}$} \\
\hline \multicolumn{5}{|c|}{$\begin{array}{l}\text { Description of what constitutes completion of this milestone: } \\
\text { Electronic transmittal to INEEL by midnight on Apri I I, 2000. and formal } \\
\text { transmitta\} of a completion letter to DOE-RL constitutes completion. }\end{array}$} \\
\hline
\end{tabular}


HNF-SP-1112, Rev. 4

PHMC

MILESTONE DESCRIPTION SHEET

\begin{tabular}{|c|c|c|c|c|}
\hline \multicolumn{4}{|c|}{$\begin{array}{l}\text { TitTe: ISSUE ANNUAL NONRADIOACTIVE AIRBORNE } \\
\text { EMISSIONS REPT (FY 2000-2046 }\end{array}$} & Date: $8 / 25 / 98$ \\
\hline \multicolumn{4}{|c|}{ Assigned To: WO ADAIR } & CIN: \\
\hline \multicolumn{4}{|c|}{ Program WBS Designator: 1.8 .2 .3 .8} & Due Date: $4 / 01 / 00$ \\
\hline \multicolumn{5}{|c|}{ PBS No: RL-0T01 } \\
\hline \multicolumn{2}{|c|}{ MC \#: ECP-00-802 } & TPA No: & & Rev: 0 \\
\hline $\begin{array}{l}\text { MILESTONE } \\
\text { LEVEL: } \\
\times \text { DOE-HO } \\
\text { DOE-RL } \\
\text { DOE-FO } \\
\text { CONTRACTOR }\end{array}$ & $\begin{array}{l}\text { MILESTONE } \\
\text { TYPE: } \\
\text { X EA } \\
\text { PEG } \\
\text { OTHER } \\
\text { TIP }\end{array}$ & $\begin{array}{l}\text { DNFSB STATUS: } \\
\text { DNFSB }(Y / N): N \\
\text { COMMIT \#: } \\
\text { RECOMMM \#: }\end{array}$ & $\begin{array}{l}\text { DELIVERABLE: } \\
\text { X Report } \\
\text { Letter } \\
\text { Drawing(s) } \\
\text { Dther (Specify) }\end{array}$ & $\begin{array}{l}\text { ADDRESS TO: } \\
\text { DOE-HQ } \\
\times \text { DOE-RL } \\
x \text { Other (Specify) } \\
\text { WASH STATE DEPT } \\
\text { OF ECOLOGY }\end{array}$ \\
\hline \multicolumn{5}{|c|}{$\begin{array}{l}\text { Milestone Description: } \\
\text { The nonradioactive airborne emissions report contains information on BHI } \\
\text { and FDH managed operations having the potential to emit combustion products } \\
\text { from fossil fuels and inorganic compounds from waste storage tanks. } \\
\text { Preparation of this report requires that nonradioactive airborne emissions } \\
\text { data wi77 be recorded on emissions inventory forms. Completed inventory } \\
\text { forms wi7l be provided to DoE-RL for transmittal to the Washington State } \\
\text { Department of Ecology. }\end{array}$} \\
\hline \multicolumn{5}{|c|}{$\begin{array}{l}\text { Description of what constitutes completion of this milestone: } \\
\text { Formal transmittal of the report to DOE-RL constitutes completion. DOE-RL } \\
\text { must transmit this report to arrive at Ecology by April 15.2000. }\end{array}$} \\
\hline
\end{tabular}




\section{PHMC \\ MILESTONE DESCRIPTION SHEET}

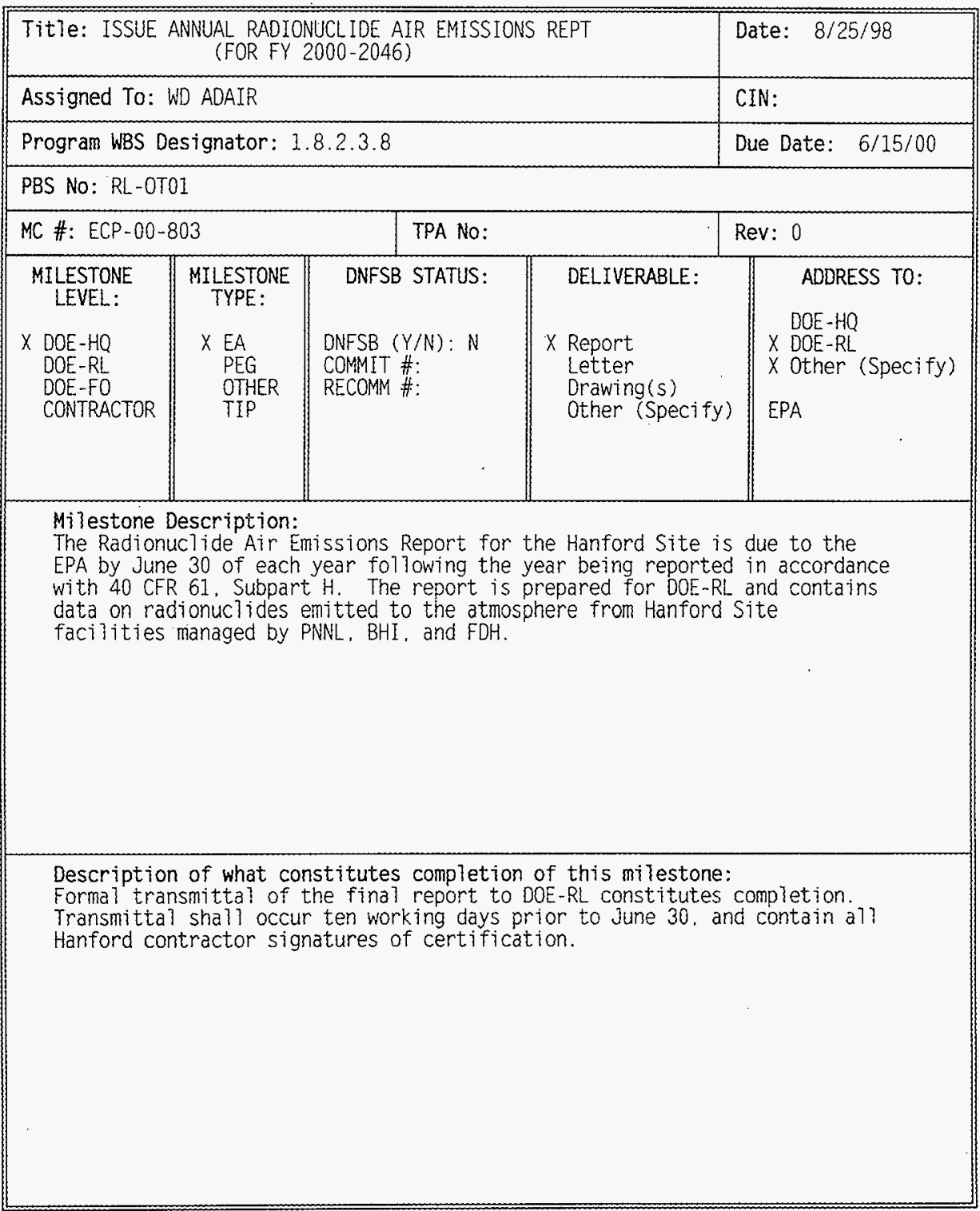




\section{PHMC}

MILESTONE DESCRIPTION SHEET

\begin{tabular}{|c|c|c|c|c|}
\hline \multicolumn{4}{|c|}{$\begin{array}{l}\text { Title: ISSUE ANNUAL REPORT ON ENV. RELEASES (FOR FY } \\
2000-2046 \text { ) }\end{array}$} & Date: $8 / 25 / 98$ \\
\hline \multicolumn{4}{|c|}{ Assigned TO: WD ADAIR } & CIN: \\
\hline \multicolumn{4}{|c|}{ Program WBS Designator: $1 \cdot 8 \cdot 2.3 .8$} & Due Date: $8 / 31 / 00$ \\
\hline \multicolumn{5}{|c|}{ PBS No: RL-OT01 } \\
\hline \multicolumn{2}{|c|}{ MC \#: ECP-00-804 } & TPA No: & & Rev: 0 \\
\hline $\begin{array}{l}\text { MILESTONE } \\
\text { LEVEL: } \\
\text { X DOE-HO } \\
\text { DOE-RL } \\
\text { DOE-FO } \\
\text { CONTRACTOR }\end{array}$ & $\begin{array}{l}\text { MILESTONE } \\
\text { TYPE: } \\
\times \text { EA } \\
\text { PEG } \\
\text { OTHER } \\
\text { TIP }\end{array}$ & $\begin{array}{l}\text { DNFSB STATUS: } \\
\text { DNFSB }(Y / N): N \\
\text { COMMIT \#: } \\
\text { RECOMM \#: }\end{array}$ & $\begin{array}{l}\text { DELIVERABLE: } \\
\text { X Report } \\
\text { Letter } \\
\text { Orawing(s) } \\
\text { Other (Specify) }\end{array}$ & $\begin{array}{l}\text { ADDRESS TO: } \\
\text { DOE-HQ } \\
\times \text { DOE-RL } \\
\text { Other (Specify) }\end{array}$ \\
\hline \multicolumn{5}{|c|}{$\begin{array}{l}\text { Milestone Description: } \\
\text { This annual report presents data for radioactive and nonradioactive } \\
\text { substances released into the environment during the previous calendar year } \\
\text { from the Hanford Site facilities managed by FDH and BHI. Both summary and } \\
\text { detailed presentations of this data are given. and some comparisons to data } \\
\text { from previous years are made. Although not an enforceable agreement } \\
\text { milestone. it does document compliance with DOE Order } 5400.1 \text {. }\end{array}$} \\
\hline \multicolumn{5}{|c|}{$\begin{array}{l}\text { Description of what constitutes completion of this milestone: } \\
\text { This report is not transmitted by DOE-RL to other agencies. Formal } \\
\text { transmittal of the report to DOE-RL by August } 31.2000 \text {. with contractor } \\
\text { signatures of approval constitutes completion. }\end{array}$} \\
\hline
\end{tabular}




\section{PHMC}

\section{MILESTONE DESCRIPTION SHEET}

\begin{tabular}{|c|c|c|c|c|}
\hline \multicolumn{4}{|c|}{$\begin{array}{c}\text { TitTe: ISSUE REPT OF NEAR-FACILITY ENV MONITORING } \\
\text { (FOR FY 2000-2046) }\end{array}$} & Date: $8 / 25 / 98$ \\
\hline \multicolumn{4}{|c|}{ Assigned To: WD ADAIR } & CIN: \\
\hline \multicolumn{4}{|c|}{ Program WBS Designator: $1.8 \cdot 2.3 .8$} & Due Date: $7 / 30 / 00$ \\
\hline \multicolumn{5}{|c|}{ PBS No: RL-0TO1 } \\
\hline \multicolumn{2}{|c|}{ MC \#: ECP-00-805 } & TPA No: & & Rev: 0 \\
\hline $\begin{array}{l}\text { MILESTONE } \\
\text { LEVEL: } \\
\text { DOE-HO } \\
\times \text { DOE-RL } \\
\text { DOE-FO } \\
\text { CONTRACTOR }\end{array}$ & $\begin{array}{l}\text { MILESTONE } \\
\text { TYPE: } \\
\text { EA } \\
\text { PEG } \\
\times \text { OTHER } \\
\text { TIP }\end{array}$ & $\begin{array}{l}\text { DNFSB STATUS: } \\
\text { DNFSB }(Y / N): N \\
\text { COMMIT \#: } \\
\text { RECOMM \#: }\end{array}$ & $\begin{array}{l}\text { DELIVERABLE: } \\
\text { X Report } \\
\text { Letter } \\
\text { Orawing(s) } \\
\text { Other (Specify) }\end{array}$ & $\begin{array}{l}\text { ADDRESS TO: } \\
\text { DOE-HQ } \\
\times \text { DOE-RL } \\
\text { Other (Specify) }\end{array}$ \\
\hline \multicolumn{5}{|c|}{$\begin{array}{l}\text { Milestone Description: } \\
\text { The monitoring program consists of collecting and analyzing environmental } \\
\text { samples and methodically surveying areas near facilities releasing } \\
\text { effluents and waste streams. The program also evaluates acquired } \\
\text { analytical data, determines the effectiveness of facility effluent } \\
\text { monitoring and detects and monitors unusual conditions. This milestone } \\
\text { complies with ooE Orders } 5400.1 .5484 .1 .5400 .5 \text { and } 5820.2 \mathrm{~A} \text { and } 10 \mathrm{CFR} 835 \text {. }\end{array}$} \\
\hline \multicolumn{5}{|c|}{$\begin{array}{l}\text { Description of what constitutes completion of this milestone: } \\
\text { This rpeort is not transmitted by DOE-RL to other agencies. Formal } \\
\text { transmittal of the report to DOE-RL by July 30.2000, with contractor } \\
\text { signatures of approval constitutes completion. }\end{array}$} \\
\hline
\end{tabular}


HNF-SP-1112, Rev. 4

PHMC

MILESTONE DESCRIPTION SHEET

\begin{tabular}{|c|c|c|c|c|}
\hline \multicolumn{4}{|c|}{$\begin{array}{l}\text { TitTe: ISSUE THIRD QUARTER NESHAP STATUS (FOR FY } \\
\text { 2000-2046) }\end{array}$} & Date: $8 / 25 / 98$ \\
\hline \multicolumn{4}{|c|}{ Assigned To: WD ADAIR } & CIN: \\
\hline \multicolumn{4}{|c|}{ Program WBS Designator: 1.8.2.3.9 } & Due Date: $10 / 23 / 99$ \\
\hline \multicolumn{5}{|c|}{ PBS NO: RL-OTO1 } \\
\hline \multicolumn{2}{|c|}{ MC \#: ECP-00-901 } & TPA No: & & Rev: 0 \\
\hline $\begin{array}{l}\text { MILESTONE } \\
\text { LEVEL: } \\
\times \text { DOE-HQ } \\
\text { DOE-RL } \\
\text { DOE-FO } \\
\text { CONTRACTOR }\end{array}$ & $\begin{array}{l}\text { MILESTONE } \\
\text { TYPE: } \\
\times \text { EA } \\
\text { PEG } \\
\text { OTHER } \\
\text { TIP }\end{array}$ & $\begin{array}{l}\text { DNFSB STATUS: } \\
\text { DNFSB }(Y / N): N \\
\text { COMMIT \#: } \\
\text { RECOMM \#: }\end{array}$ & $\begin{array}{l}\text { DELIVERABLE: } \\
\\
\text { Report } \\
\times \text { Letter } \\
\text { Drawing(s) } \\
\text { Other (Specify) }\end{array}$ & $\begin{array}{l}\text { ADDRESS TO: } \\
\text { DOE-HO } \\
\times \text { DOE-RL } \\
\times \text { Other (Specify) } \\
\text { EPA }\end{array}$ \\
\hline \multicolumn{5}{|c|}{$\begin{array}{l}\text { Milestone Description: } \\
\text { This milestone is a quarterly report on the progress made towards } \\
\text { satisgying the FFCA requirements. This quarterly report is required as an } \\
\text { update on Hanford site activities performed in support of the FFCA. }\end{array}$} \\
\hline \multicolumn{5}{|c|}{$\begin{array}{l}\text { Description of what constitutes completion of this milestone: } \\
\text { This milestone is completed by delivering the report to DOE-RL and } \\
\text { providing a telefax transmittal to EPA. The report is subsequently } \\
\text { transmitted by DOE-RL to the regulators by November } 6 \text {. } 1999 \text {. }\end{array}$} \\
\hline
\end{tabular}


HNF-SP-1112, Rev. 4

\section{PHMC}

\section{MILESTONE DESCRIPTION SHEET}

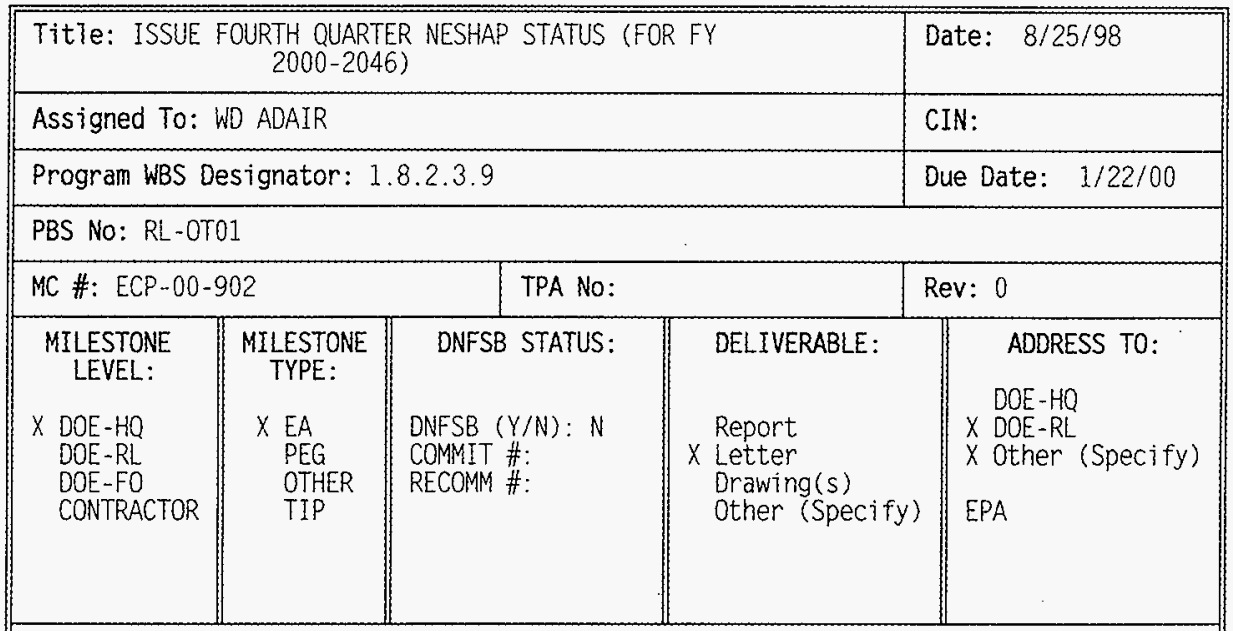

Milestone Description:

This milestone is a quarterly report on the progress made towards satisfying the FFCA requirements. This quarterly report is required as an update on Hanford Site activities performed in support of the FFCA.

Description of what constitutes completion of this milestone:

This milestone is completed by delivering the report to DOE-RL and providing a telefax transmittal to EPA. This report is subsequentiy transmitted by DOE-RL to the regulators by February 5, 2000. 


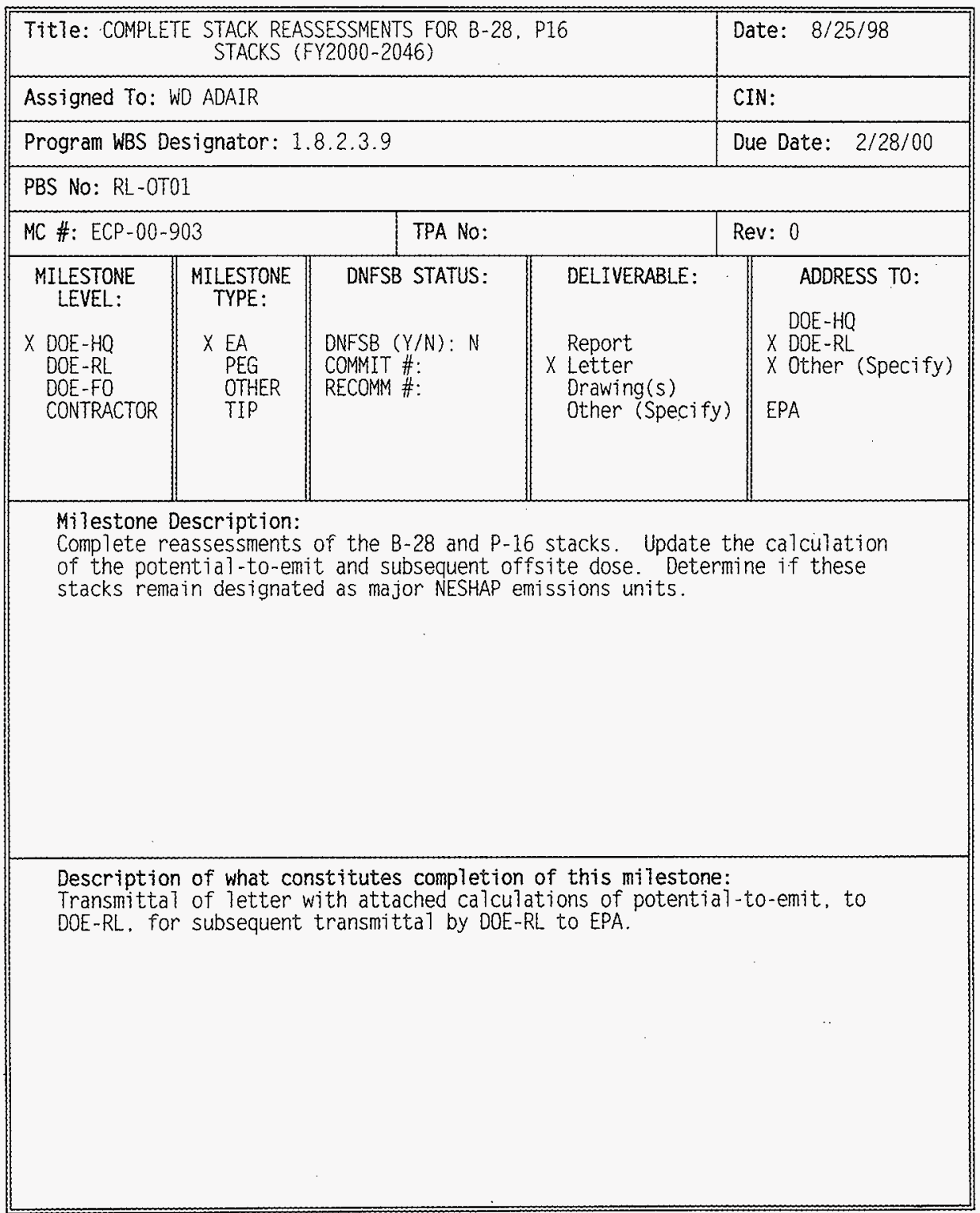


HNF-SP-1112, Rev. 4

\section{PHMC}

MILESTONE DESCRIPTION SHEET

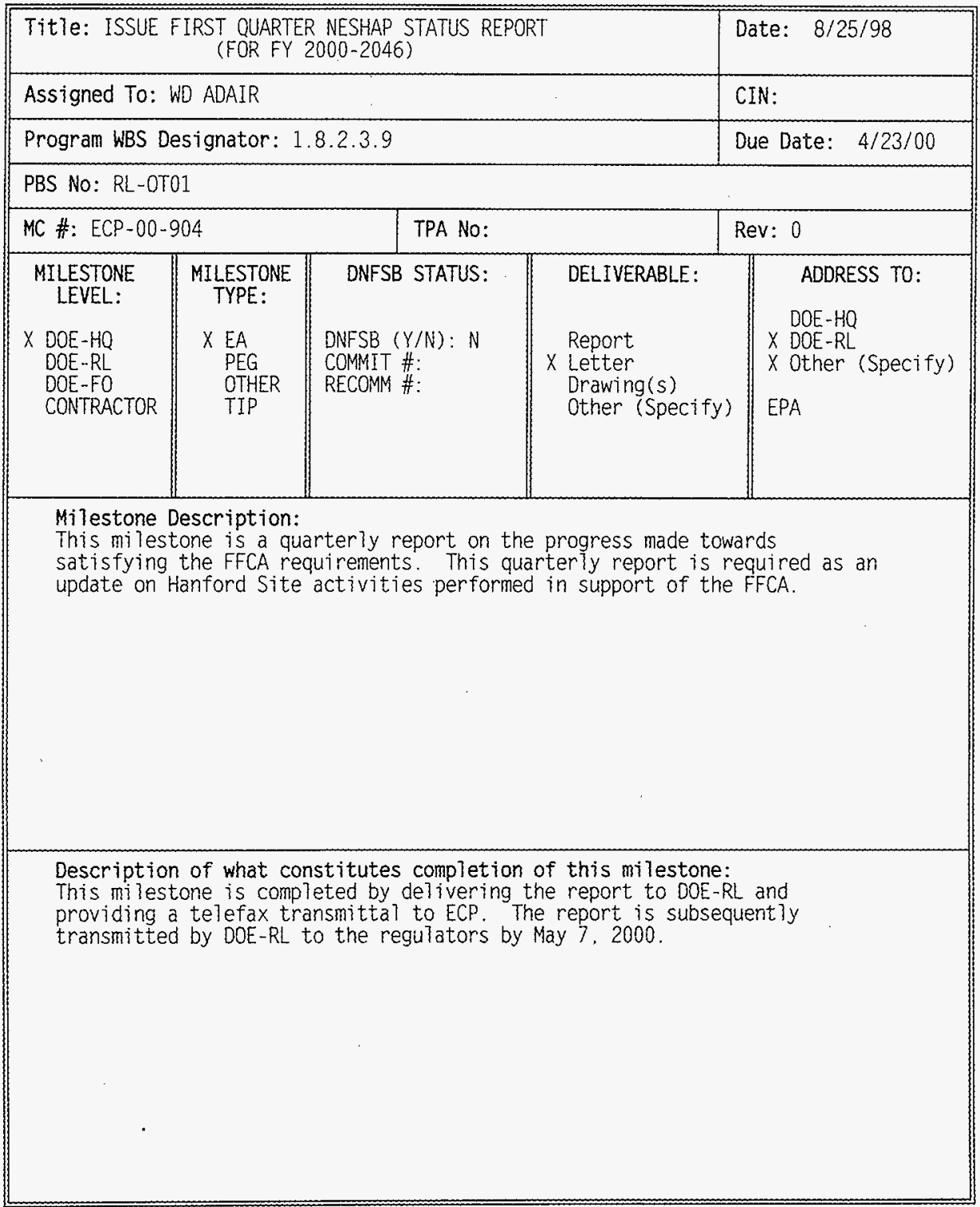




\section{PHMC}

\section{MILESTONE DESCRIPTION SHEET}

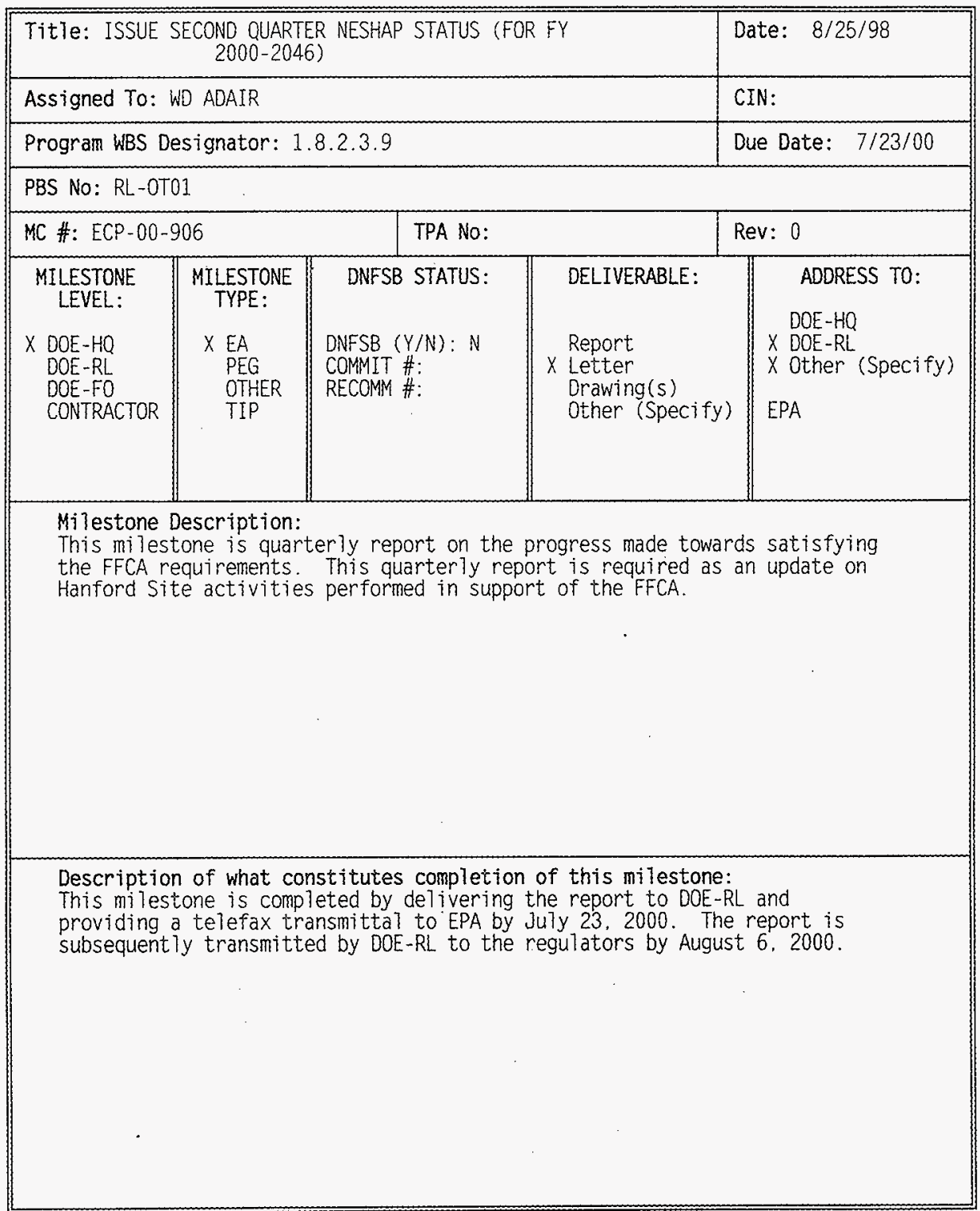


HNF-SP-1112, Rev. 4

\section{PHMC}

\section{MILESTONE DESCRIPTION SHEET}

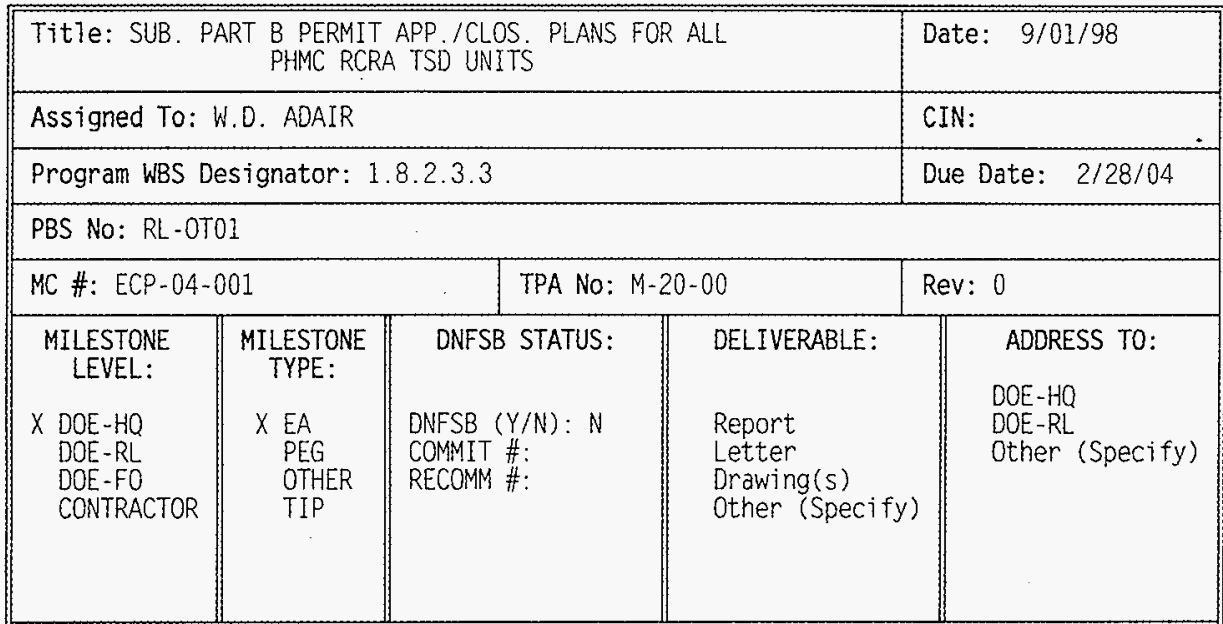

Milestone Description:

Prepare Part B Permit applications or closure/postclosure plans for all

PHMC-operated RCRA TSD units. Plans will be submitted to Ecology for approval. Preclosure work plans will be prepared and submitted for approval for TSD units which will achieve closure in conjunction with the disposition of the facility in which they are contained.

Description of what constitutes completion of this milestone:

Submittal to RL the final RCRA TSD unit for subsequent transmittal to Ecology. 


\section{PHMC \\ MILESTONE DESCRIPTION SHEET}

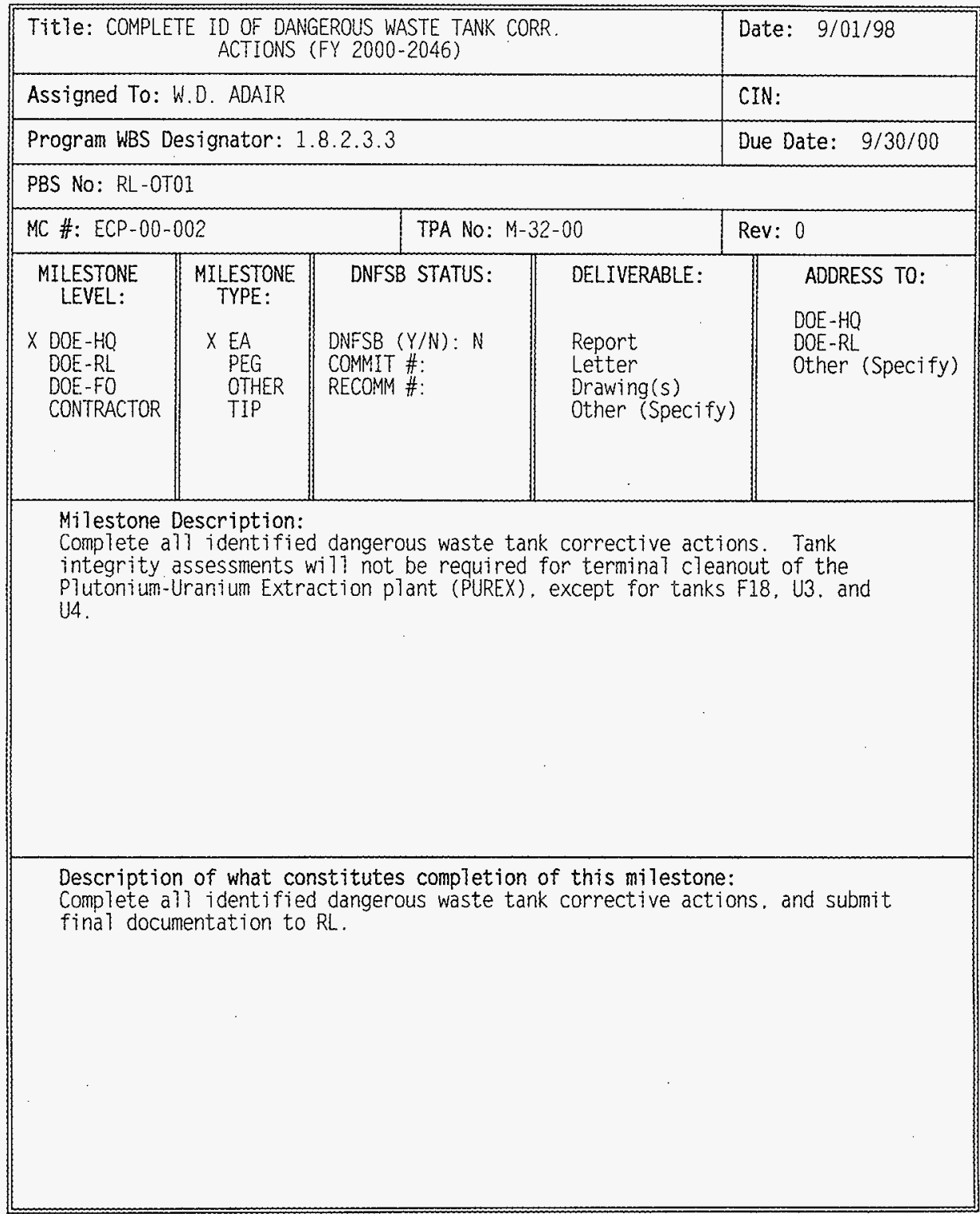




\section{PHMC}

\section{MILESTONE DESCRIPTION SHEET}

\begin{tabular}{|c|c|c|c|c|}
\hline \multicolumn{4}{|c|}{$\begin{array}{l}\text { Title: BIENNIAL ASSESS. OF INFO \& DATA ACCESS } \\
\text { NEEDS EPA/ECO }(2000-2046)\end{array}$} & Date: $9 / 01 / 98$ \\
\hline \multicolumn{4}{|c|}{ Assigned To: W.D. ADAIR } & CIN: \\
\hline \multicolumn{4}{|c|}{ Program WBS Designator: 1.8 .2 .3 .6} & Due Date: $3 / 31 / 00$ \\
\hline \multicolumn{5}{|c|}{ PBS No: RL-OT01 } \\
\hline \multicolumn{2}{|c|}{ MC \#: ECP-00-003 } & \multicolumn{2}{|c|}{ TPA No: $M-35-090$} & Rev: 0 \\
\hline $\begin{array}{l}\text { MILESTONE } \\
\text { LEVEL: }\end{array}$ & $\begin{array}{l}\text { MILESTONE } \\
\text { TYPE: }\end{array}$ & DNFSB STATUS: & DELIVERABLE: & \multirow{2}{*}{$\begin{array}{l}\text { ADDRESS TO: } \\
\text { DOE-HQ } \\
\text { DOE-RL } \\
\text { Other (Specify) }\end{array}$} \\
\hline $\begin{array}{l}X \text { DOE-HQ } \\
\text { DOE-RL } \\
\text { DOE-FO } \\
\text { CONTRACTOR }\end{array}$ & $\begin{array}{l}X \text { EA } \\
\text { PEG } \\
\text { OTHER } \\
\text { TIP }\end{array}$ & $\begin{array}{l}\text { DNFSB }(Y / N): N \\
\text { COMMIT \#: } \\
\text { RECOMM \#: }\end{array}$ & $\begin{array}{l}\text { Report } \\
\text { Letter } \\
\text { Drawing(s) } \\
\times \text { Other (Specify) } \\
\text { TPA MILESTONE }\end{array}$ & \\
\hline
\end{tabular}

Milestone Description:

Conduct a biennial assessment of information and data access needs with EPA and Ecology

Description of what constitutes completion of this milestone:

Propose to RL implementing schedules (Tri-Party Agreement milestones) for enhancements as a result of biennial assessments starting March 31, 2000 


\subsection{Cost Baseline}

This section contains data for life cycle costs by Project Baseline Summary (PBS) and life cycie Budget Authority (B/A) by PBS, and execution year cost baseline by month by PBS.

\subsection{Life Cycle Cost Baseline (BCWS) by PBS}

The data in Exhibit 1a provides Life Cycle BCWS by fund type by PBS from fiscal year 1998 through project completion. Management Reserve held by RL is not included in the BCWS baseline total. Estimated carryover is not reported at this time. Please see the figure on the following page. 


\section{SUPPORT}

\begin{tabular}{|c|c|c|c|c|c|c|c|c|c|c|c|}
\hline PROJECT WBS: & $\begin{array}{r}1.8 .2 .3 \\
R L-0701 \\
\end{array}$ & & & & & & & & & & \multirow{3}{*}{$\begin{array}{l}\text { SUBTOT } \\
\text { FY1997- } \\
\text { FY2006 }\end{array}$} \\
\hline PBS TITLE: & \multicolumn{10}{|c|}{ ENVIRONMENTAL COMPLIANCE } & \\
\hline FUND TYPE & EY1997 & FY1998 & FYig9g & FY2000 & FY2001 & FY2002 & FY2003 & FY2004 & FY2005 & FY2006 & \\
\hline \multirow[t]{2}{*}{ OPERATING EXPENSE } & 10,751 & 10,546 & 10.585 & 11,144 & 11,144 & 11,144 & 11,144 & 11,144 & 11,144 & 11,144 & 109,890 \\
\hline & & & 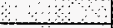 & . & & & & & & & \\
\hline \multirow[t]{2}{*}{ CENRTC } & $\therefore$ & 9 & $\therefore \therefore-2$ & - & - & - & - & - & - & - & 9 \\
\hline & & & 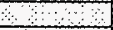 & & & & & & & & \\
\hline \multirow[t]{2}{*}{ GENERAL PLANT PRO JECT } & $=$ & 121 & 1,870 & 833 & $\therefore$ & $\therefore$ & - & $\therefore$ & $=$ & - & 2,824 \\
\hline & & & 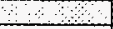 & & & & & & & & \\
\hline \multicolumn{12}{|l|}{ LINE ITEM Rist Exch Ono) } \\
\hline \multirow{6}{*}{$\frac{0}{0}$} & - & - & $\therefore$ & $\therefore$ & 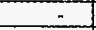 & - & - & $\because$ & - & $=$ & - \\
\hline & - & - & 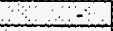 & $\therefore$ & $=$ & - & $=$ & $=$ & $\therefore$ & $=$ & $\because$ \\
\hline & - & - & $\therefore$ & - & $\div$ & $\therefore$ & - & - & - & - & $\because$ \\
\hline & - & - & $\therefore$ & - & - & - & $=$ & $\therefore$ & - & - & - \\
\hline & - & $\therefore$ & $\therefore \quad$ & - & $=$ & - & - & $\therefore$ & - & $\div$ & - \\
\hline & - & - & $\therefore$ & - & $\therefore$ & - & - & $=$ & - & - & - \\
\hline Subtotal Line fiems & - & - & . & - & - & $\div$ & - & $=$ & - & - & - \\
\hline Escalation & 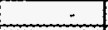 & - & $\therefore$ & 238 & 449 & 686 & 929 & 1,177 & 1,430 & 1,689 & 6,597 \\
\hline \multirow[t]{2}{*}{ TOTAL BCWSIPMB? } & 10,751 & 10,676 & 12,455 & 12,215 & 11,593 & 111,830 & 12073 & 12,321 & 412,564 & $12+833$ & 8119320 \\
\hline & . & 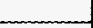 & 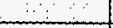 & - & & & & 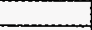 & & & \\
\hline \multirow[t]{2}{*}{ MGMT RESERVE } & - & $\therefore$ & $=$ & - & - & $\therefore$ & $\therefore$ & - & - & - & $=$ \\
\hline & & & $\therefore$ & & & & & & & & \\
\hline \multirow[t]{2}{*}{ UNE ITEM CONTINGENCY ${ }^{2}$} & - & $\therefore$ & $\therefore$ & - & $=$ & - & - & - & - & - & $\therefore$ \\
\hline & & & $\therefore \quad 1$ & & & & & & & & \\
\hline \multirow[t]{2}{*}{ TRANSFERS $^{3}$} & $=$ & + & $+\ldots$ & - & - & $\therefore$ & - & - & - & $=$ & $\therefore$ \\
\hline & & & 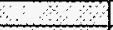 & & & & & & & & \\
\hline Subtotal & $\therefore$ & $\therefore$ & $\therefore \quad 1$ & $=$ & - & - & - & - & - & $\therefore$ & - \\
\hline Eecalation &. & - & $\therefore-\cdots$ & - & - & - & 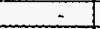 & $=$ & - & - & - \\
\hline TOTAL & 10.751 & 10.676 & 12,455 & 12,215 & 11,593 & 11,830 & 12,073 & 12321 & 12,574 & 12833 & 119,320 \\
\hline
\end{tabular}

'Buogeted Cost of Work Scheduled (BCWS) Equals Performance Measurement Baseline (PMB); Expense Canyover Is Not included ${ }^{2}$ Management Reservo and Line ltem Contingency Held By RL.

J Funds Workscope Transferted to Other Sites. Transterred to Harford from Other Sites, and/or Funds Workscope Ccntrolled by RL. 


\section{SUPPORT}

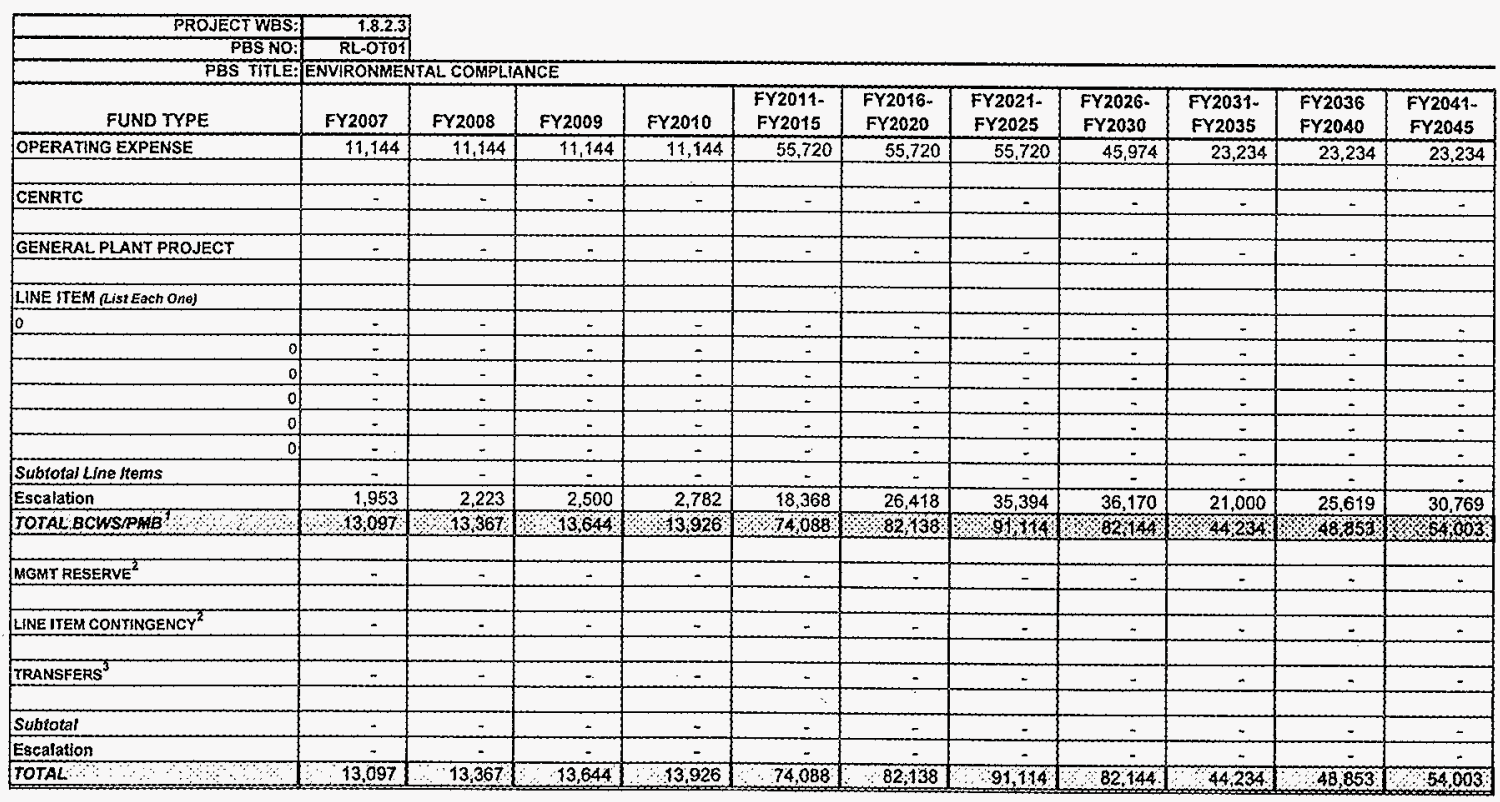

'Budgeted Cost of Work Scheduled (BCWS) Equals Performance Measurement Baseline (PMB); Expense Carrycver is Not included ${ }^{2}$ Management Reserve and Line Item Contingency Held By RL.

${ }^{3}$ FundsWorkscope Transferred to Other Sites, Transferred to Hanford from Other Sltes, and/or Funds Workscope Controlied by RL. 
EXHIBIT 1a

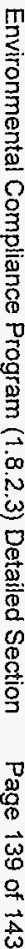

\section{LIFE CYCLE COST BASELINE (BCWS) BY YEAR BY FUND TYPE} BY PROJECT BASELINE SUMMARY (PBS)

FY 1999

(\$000s)

\begin{tabular}{|c|c|c|}
\hline $\begin{array}{r}\text { PROJECT WBS: } \\
\text { PBS NO: }\end{array}$ & & \\
\hline PBS IITLE: & & TOTAL \\
\hline FUND TYPE & $\begin{array}{l}\text { FY2046 } \\
\text { FY2050 }\end{array}$ & $\begin{array}{l}\text { FY1997. } \\
\text { FY2050 }\end{array}$ \\
\hline OPERATING EXPENSE & 23,234 & 460,536 \\
\hline CENRTC & - & 9 \\
\hline GENERAL PLANT PROJECT & - & 2,824 \\
\hline \multicolumn{3}{|l|}{ LINE ITEM Rist Each One) } \\
\hline 0 & - & - \\
\hline of & - & - \\
\hline 0 & - & - \\
\hline 0 & - & $=$ \\
\hline 0 & $=$ & $=$ \\
\hline 0. & - & 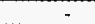 \\
\hline Sublotal Line Items & - & $\therefore$ \\
\hline Escalation & 36,511 & 246,304 \\
\hline FOTAL BCWSIPMB & 59745 & 709.672 \\
\hline MGMTRESERVE ${ }^{2}$ & - & - \\
\hline UUNE ITEM CONTINGENCY ${ }^{2}$ & - & - \\
\hline & & \\
\hline TRANSFERS ${ }^{3}$ & $=$ & $=$ \\
\hline Subtotal & - & T \\
\hline Escalation & $\ldots$ & - \\
\hline TOTAL & 59,745 & 709,672 \\
\hline
\end{tabular}


HNF-SP-1112, Rev. 4

\subsection{Life Cycle Budget Authority (B/A) by PBS}

The data in Exhibit 4a provides new B/A by fund lype by PBS for fiscal years 1998 and 1999. The carryover for FY 1999 is not included at this time. Please see the figure on the following page. 


\section{SUPPORT \\ BUDGET AUTHORITY (B/A) BY YEAR BY FUND TYPE \\ BY PROJECT BASELINE SUMMARY (PBS)}

FY 1999

(\$000s)

\begin{tabular}{|c|c|c|}
\hline PROJECT WBS: & 1.8 .2 .3 & \\
\hline PBS NO: & RL-OTO1 & \\
\hline PBS TITLE: & TAL COMPLIANCE & \\
\hline & \multirow{2}{*}{$\begin{array}{c}\text { TARGET } \\
\text { B/A } \\
\text { FY1998 }\end{array}$} & \multirow{2}{*}{$\begin{array}{l}\text { PRESIDENTS } \\
\text { BUDGET PLUS } \\
\text { CARRYOVER' } \\
\text { FY1999 }\end{array}$} \\
\hline FUND TYPE & & \\
\hline OPERATING EXPENSE & 10,076 & 10,585 \\
\hline CENRTC & 9 & - \\
\hline & & \\
\hline GENERAL PLANT PROJECT & - & 1,870 \\
\hline & & \\
\hline \multicolumn{3}{|l|}{ LINE ITEM (LIst Each One) } \\
\hline 0 & - & - \\
\hline 0 & - & - \\
\hline 0 & $\therefore$ & - \\
\hline 0 & $=$ & - \\
\hline 0 & - & - \\
\hline 0 & - & - \\
\hline Subtofal Line lfems & - & - \\
\hline TOTAL NEW B/A & 10,085 & 12,455 \\
\hline ESTIMATED FY 1998 CARRYOVER & & - \\
\hline TOTAL NEW B/A+CARRYOVER & 10,085 & 12,455 \\
\hline
\end{tabular}

'Estimated Camyover Is NOT Included in Cost Baseline; Change Request For Actual Carryover

Subrnitted After September 30, 1998 
HNF-SP-1112, Rev. 4

\subsection{Execution Year Cost Baseline by Month by PBS by Fund Type}

The data in Exhibit 2 a provides the BCWS by month for each PBS by fund type. Due to the new financial system, this cost exhibit cannot be completed at this time. it is estimated that this information will be available around November, 1998. Estimated carryover is not reported at this time. Please see the figure on the following page. 
FY 1999 COST BASELINE (BCWS) BY MONTH

BY FUND TYPE BY PROJECT BASELINE SUMRARY (PBS)

EXECUTION YEAR

(\$000s)

\begin{tabular}{|c|c|c|c|c|c|c|c|c|c|c|c|c|c|}
\hline $\begin{array}{r}\text { PROJECT (PES)WBS: } \\
\text { PBS NO: }\end{array}$ & $\begin{array}{r}1.8 .2 .3 \\
\text { RLLOT.01 } \\
\end{array}$ & & & & & & & & & & & & \\
\hline PBSTITLE: & ENVIRONME & TALCON & ANCE & & & & & & & & & & \\
\hline FUND TYPE & OCT & Nov & $\mathrm{DEC}$ & SAN & FEB & MAR & APR & MAY & JUN & SUL & AUG & SEP & TOTAL \\
\hline Operating Expense & 10,585 & $\therefore$ & - & - & - & - & $\therefore$ & $\therefore$ & - & & $\therefore$ & $\therefore$ & 10.585 \\
\hline CENRTC & . & $\dot{0}$ & 4 & - & $\therefore$ & $\therefore$ & - & . & - & . & . & $\therefore$ & - \\
\hline GPP & 1.870 & $\therefore$ & - & - & - & - & $\therefore$ & - & - & - & - & - & 1.870 \\
\hline Line Item & $\dot{.}$ & $\therefore$ & $=$ & - & $\therefore$ & - & $\therefore$ & $\therefore$ & - & - & . & - & - \\
\hline 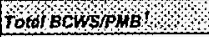 & 12,455 & 4 & 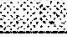 & 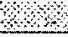 & 8 & 2 & 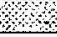 & \% & 4 & 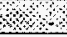 & 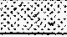 & 4 & 12,465 \\
\hline Mgmt Reservg ${ }^{2}$ & - & $\therefore$ & $\therefore$ & $\therefore$ & $\therefore$ & - & . & - & $\therefore$ & - & . & . & \\
\hline Line stom Conthngency ${ }^{2}$ & - & . & - & - & . & . & $\therefore$ & . & - & $\therefore$ & $\therefore$ & . & . \\
\hline Transfers' & - & - & - & - & - & - & . & $\because$ & $\therefore$ & $\therefore$ & $\dot{.}$ & - & $=$ \\
\hline rotal & 12.455 & . & - & $=$ & $\therefore$ & $=$ & - & $=$ & $\therefore$ & - & - & . & 12,455 \\
\hline
\end{tabular}

'Budgilad Cost of Work Schadulod (BCWS) Equais Porfomanco Mosstrament Basoling (PMB); Expensa Carnover is Not included

${ }^{2} \mathrm{M}$ ansgement Rosorve and Lina !larn Contingoncy hidd By Rt

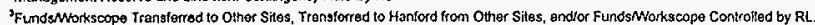


HNF-SP-1112, Rev, 4

TABLE OF CONTENTS

ADDITIONAL REQUIREMENTS SECTION

Page

1.1 Project Priority List.....

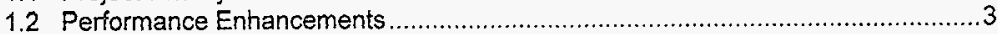

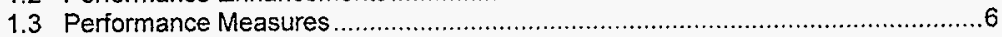

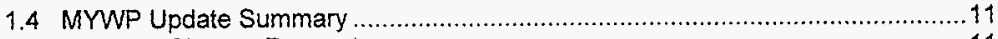

1.5 Baseline Change Request ............................................................................. 


\subsection{Project Priority List}

The project priority list displays the president's budget for FY1999 and the target funding level for FY2000 for the two Units of Analysis (UOA) that comprise the Environmental Compliance Program. These two UOAs are Effluent and Environmental Monitoring, classified as "Min Safe"; and Hanford Environmental Management, classified as "Essential Services". The project priority list is on the following page. 
HNF-SP-1112, Rev. 4

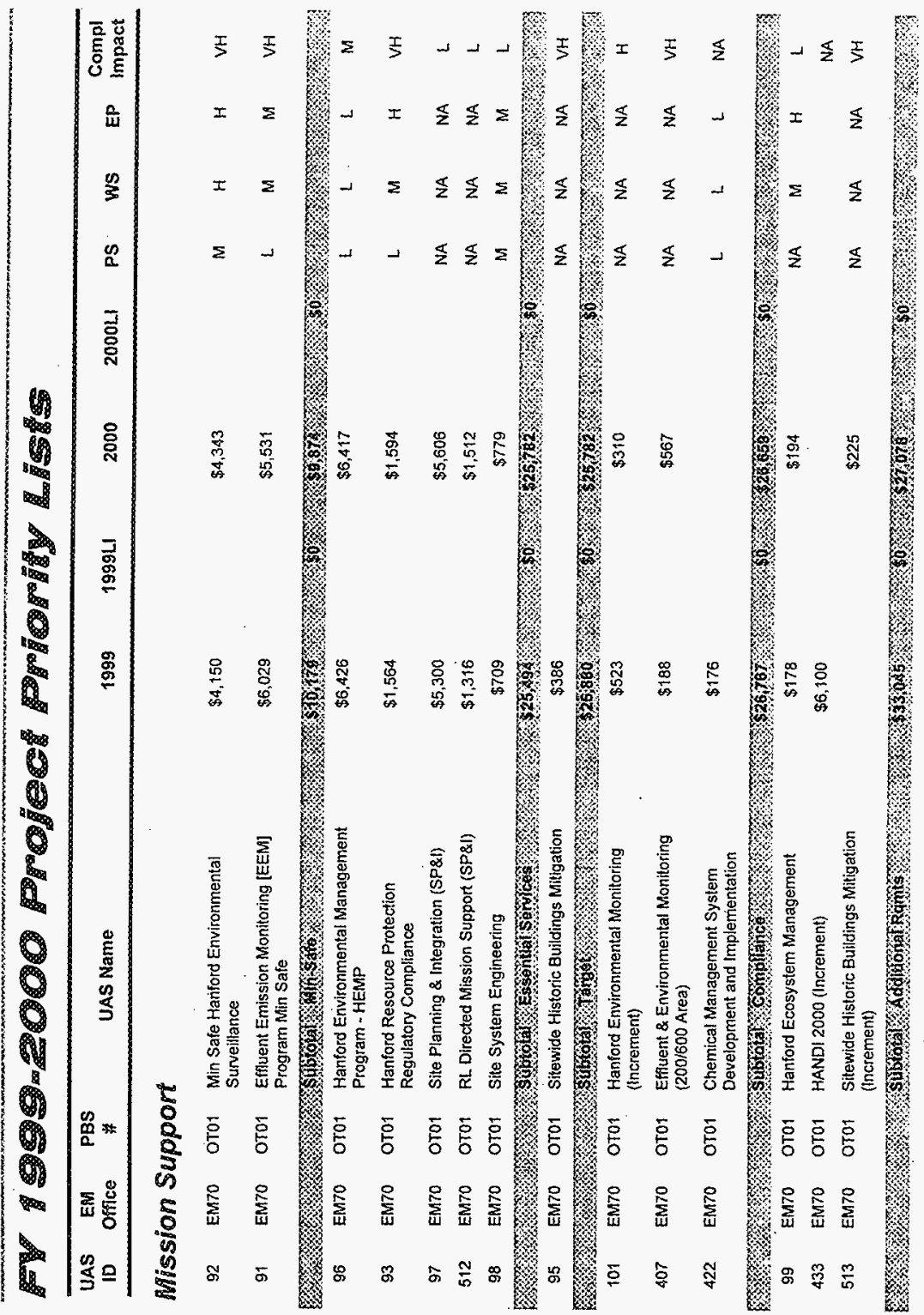




\subsection{Performance Enhancements}

The project has made an assessment, and there is not funding available in the current President's baseline to accelerate/enhance lifecycle work scope at this time. Please see the forms on the following pages. 
PERFORMANCE ENHANCEMENT WORKSHEET

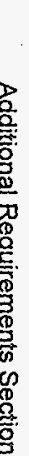

ITEM 1

Part!

PROS:

.

OESRIPTONOE ENMANCEMENT OPPORTUNTT

Ranges

PROBAEILITY LIFE CYCLE

FUNDING REOURED

TO INVESTIGATE

WBSt(s) WHERE OF SUCCESS $\%$ / SAVINGS (SMillions) OPPORTUNITY (SThous) FUNDING IS REQUIRED Totan Totar
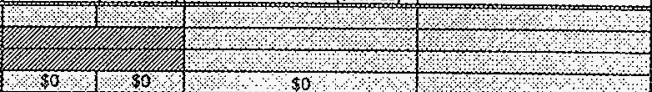

Part II

STEPSISCHEDULE TO ACCOMPLISH PART I ENHANCEMENTS

莡

\section{STEPSISCHEDULE TOACCOMPLISH PART IENHANCEMENTS}

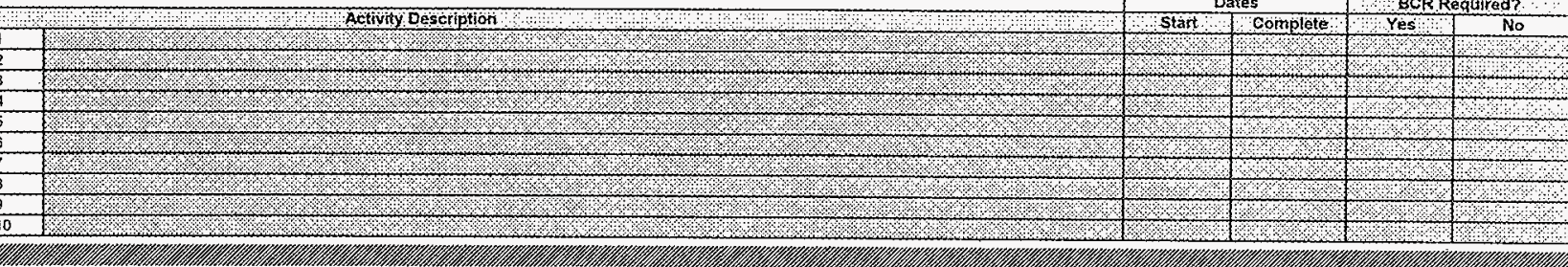

ITEM 2

Part I

PRoj:

prs:

8s: $\$$

DESCRIPTION OF ENHANCENENT OPPORTUNIT

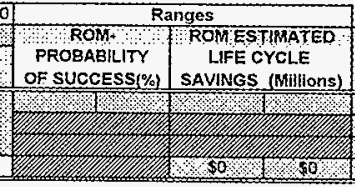

FUNDING REQUIRED

TO INVESTIGATE OPPORTUNITY (SThOLS)

WBSF( FUNDING IS REQUIRED

\section{Part ii}

STEPS/SGHEDULE TO ACCOMPLISH PART I ENHANCEMENTS
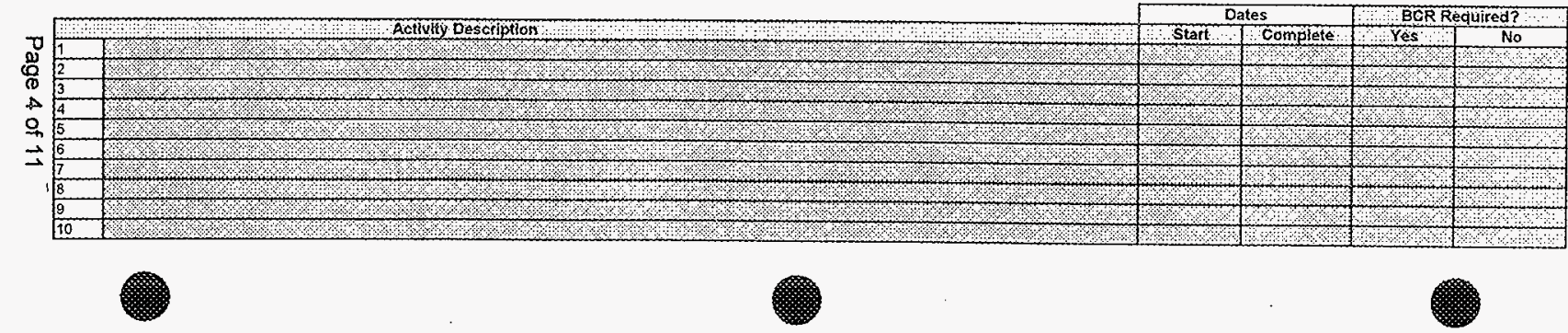
PERFORMANCE ENHANCEMENT WORKSHEET

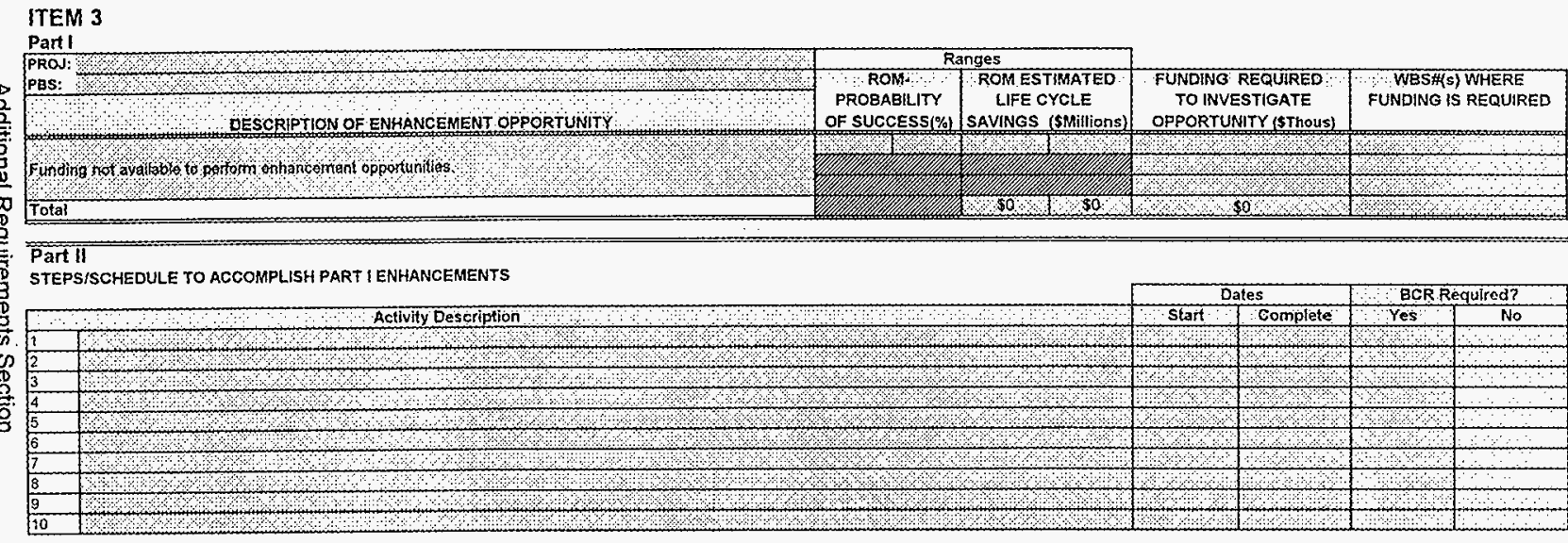

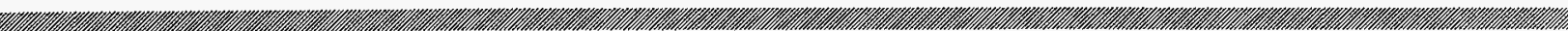

\section{ITEM 4}

Part!

PROS:

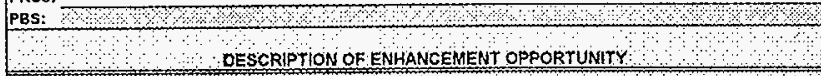

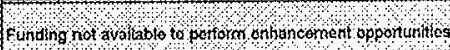

Iotal

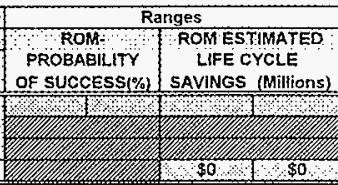

FUNDING REQUURED

TO INVESTIGATE

WESF(S) WHERE OPPORTUNITY (STHOUS) FUNDING IS REQUIRED

\section{Part II}

STEPS/SCHEDULE TO ACCOMPLISH PART I ENHANCEMENTS

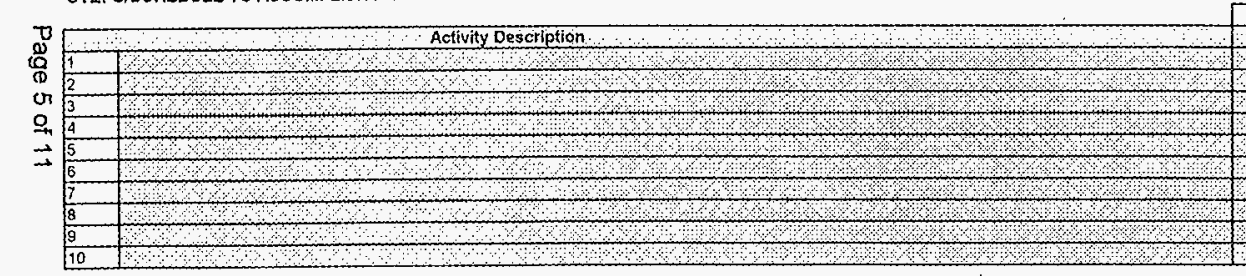

\begin{tabular}{|r|r|r|r|}
\hline \multicolumn{2}{|c|}{ Dates } & BCR Required? \\
\hline Start & Complate & Yes & No \\
\hline & & & \\
\hline & & & \\
\hline & & & \\
\hline & & & \\
\hline & & & \\
\hline & & & \\
\hline & & & \\
\hline & & & \\
\hline
\end{tabular}


HNF-SP-1112, Rev. 4

\subsection{Performance Measures}

The Environmental Compliance Program will have performance objectives in FY99, but will not have performance measures. There will be a project execution plan however for the Environmental Compliance Program. The performance objective table is on the following page. 
Performance Objectives and Measures

Environmentał Compliance Program

\begin{tabular}{|c|c|c|c|c|}
\hline $\begin{array}{l}\text { HSP Success } \\
\text { indicator/Critical } \\
\text { Success }\end{array}$ & Strategic Outcome/Goal & Performance Objective & Output/Metric & $\begin{array}{l}\text { ECP } \\
\text { Management } \\
\text { Commitment }\end{array}$ \\
\hline \multirow[t]{3}{*}{ QT01-EM } & $\begin{array}{l}\text { Implement an integrated ES\&H } \\
\text { management system; } \\
\text { reduce/eliminate } \\
\text { emissions/effluent; regulatory } \\
\text { and TPA compliance }\end{array}$ & $\begin{array}{l}\text { Integration of ES\&H into } \\
\text { management and work } \\
\text { practices at all levels } \\
\text { (institutional, facility and } \\
\text { activity) to ensure the Hanford } \\
\text { Site missions are } \\
\text { accomplished while protecting } \\
\text { the public, the worker and the } \\
\text { environment }\end{array}$ & $\begin{array}{l}\text { Submit the Hanford Site Radionuclide } \\
\text { Air Emissions Report to EPA each year } \\
\text { following the year being reported. The } \\
\text { report contains data on atmospheric } \\
\text { radionuclides emitted from Hanford } \\
\text { Site facilities. }\end{array}$ & $09 / 30 / 99$ \\
\hline & & & $\begin{array}{l}\text { Submit the Hanford Site Radionuclide } \\
\text { Air Emissions Report to EPA each year } \\
\text { following the year being reported. The } \\
\text { report contains data on aimospheric } \\
\text { radionuclides emitted from Hanford } \\
\text { Site facilities. }\end{array}$ & $9 / 30 / 2000$ \\
\hline & & & $\begin{array}{l}\text { Submit the Hanford Site Radionuclide } \\
\text { Air Emissions Report to EPA each year } \\
\text { following the year being reported. The } \\
\text { report contains data on atmospheric } \\
\text { radionuclides emitted from Hanford } \\
\text { Site facilities. }\end{array}$ & $9 / 30 / 2001$ \\
\hline
\end{tabular}


Performance Objectives and Measures

Environmental Compliance Program

\begin{tabular}{|c|c|c|c|c|}
\hline $\begin{array}{l}\text { HSP Success } \\
\text { Indicator/Critical } \\
\text { Success }\end{array}$ & Strategic Outcome/Goal & Performance Objective & Output/Metric & $\begin{array}{l}\text { ECP } \\
\text { Management } \\
\text { Commitment }\end{array}$ \\
\hline OTO1-EM & $\begin{array}{l}\text { Implement an integrated ES\&H } \\
\text { management system; } \\
\text { reduce/eliminate } \\
\text { emissions/effiuent; regulatory } \\
\text { and TPA compliance }\end{array}$ & $\begin{array}{l}\text { Integration of ES\&H into } \\
\text { management and work } \\
\text { practices at afl levels } \\
\text { (institutional, facility and } \\
\text { activity) to ensure the Hanford } \\
\text { Site missions are } \\
\text { accomplished while protecting } \\
\text { the public, the worker and the } \\
\text { environment }\end{array}$ & $\begin{array}{l}\text { Submit the Tier } 2 \text { Emergency \& } \\
\text { Hazardous Chemical Inventory Report } \\
\text { to RL \& coordinate preparation/review } \\
\text { activities so RL can transmit it to the } \\
\text { WA State Emergency Response } \\
\text { Commission, local emergency planning } \\
\text { committees, \& local fire departments }\end{array}$ & $9 / 30 / 2001$ \\
\hline & & & $\begin{array}{l}\text { Submit the Tier } 2 \text { Emergency \& } \\
\text { Hazardous Chemical Inventory Report } \\
\text { to RL \& coordinate preparation/review } \\
\text { activities so RL can transmit it to the } \\
\text { WA State Emergency Response } \\
\text { Commission, local emergency planning } \\
\text { committees, \& local fire departments }\end{array}$ & $9 / 30 / 99$ \\
\hline & & & $\begin{array}{l}\text { Submit the Tier } 2 \text { Emergency \& } \\
\text { Hazardous Chemical Inventory Report } \\
\text { to RL \& coordinate preparation/review } \\
\text { activities so RL can transmit it to the } \\
\text { WA State Emergency Response } \\
\text { Commission, local emergency planning } \\
\text { committees, \& local fire departments }\end{array}$ & $9 / 30 / 2000$ \\
\hline
\end{tabular}


Performance Objectives and Measures

Environmental Compliance Program

\begin{tabular}{|c|c|c|c|c|}
\hline $\begin{array}{l}\text { HSP Success } \\
\text { Indicator/Critical } \\
\text { Success }\end{array}$ & Strategic Outcome/Goal & Performance Objective & Output/Metric & $\begin{array}{l}\text { ECP } \\
\text { Management } \\
\text { Commitment }\end{array}$ \\
\hline \multirow[t]{3}{*}{ QTQ1-EM } & $\begin{array}{l}\text { Implement an integrated ES\&H } \\
\text { management system; } \\
\text { reduce/eliminate } \\
\text { emissions/effluent; regulatory } \\
\text { and TPA compliance }\end{array}$ & $\begin{array}{l}\text { Integration of ES\&H into } \\
\text { management and work } \\
\text { practices at all levels } \\
\text { (institutional, facility and } \\
\text { activity) to ensure the Hanford } \\
\text { Site missions are } \\
\text { accomplished while protecting } \\
\text { the public, the worker and the } \\
\text { environment }\end{array}$ & $\begin{array}{l}\text { Update NEPA Source Guide } \\
\text { information and issue revised } \\
\text { reference. Place Source Guide on the } \\
\text { Hanford Intranet }\end{array}$ & $9 / 30 / 99$ \\
\hline & & & $\begin{array}{l}\text { Update NEPA Source Guide } \\
\text { information and issue revised } \\
\text { reference. Place Source Guide on the } \\
\text { Hanford Intranet }\end{array}$ & $9 / 30 / 2000$ \\
\hline & & & $\begin{array}{l}\text { Update NEPA Source Guide } \\
\text { information and issue revised } \\
\text { reference. Place Source Guide on the } \\
\text { Hanford Intranet }\end{array}$ & $09 / 30 / 01$ \\
\hline
\end{tabular}


Performance Objectives and Measures

Environmental Compliance Program

\begin{tabular}{|c|c|c|c|c|}
\hline $\begin{array}{l}\text { HSP Success } \\
\text { Indicator/Critical } \\
\text { Success }\end{array}$ & Strategic Outcome/Goal & Performance Objective & OutputMetric & $\begin{array}{l}\text { ECP } \\
\text { Management } \\
\text { Commitment }\end{array}$ \\
\hline \multirow[t]{3}{*}{ QT01-EM } & $\begin{array}{l}\text { Implement an integrated ES\&H } \\
\text { management system; } \\
\text { reduce/eliminate } \\
\text { emissions/effluent; regulatory } \\
\text { and TPA compliance }\end{array}$ & $\begin{array}{l}\text { Integration of ES\&H into } \\
\text { management and work } \\
\text { practices at all levels } \\
\text { (institutional, facility and } \\
\text { activity) to ensure the Hanford } \\
\text { Site missions are } \\
\text { accomplished while protecting } \\
\text { the public, the worker and the } \\
\text { environment }\end{array}$ & $\begin{array}{l}\text { Visually inspect } 100 / 200 / 300 / 400 / 1100 \\
\text { areas \& Columbia River bank for } \\
\text { malfunctions, deteriorations, operator } \\
\text { errors, and discharges that could } \\
\text { cause or lead to the release of } \\
\text { dangerous waste constituents to the } \\
\text { environment or could threaten human } \\
\text { life }\end{array}$ & $9 / 30 / 99$ \\
\hline & & & $\begin{array}{l}\text { Visually inspect } 100 / 200 / 300 / 400 / 1100 \\
\text { areas \& Columbia River bank for } \\
\text { malfunctions, deteriorations, operator } \\
\text { errors, and discharges that could } \\
\text { cause or lead to the release of } \\
\text { dangerous waste constituents to the } \\
\text { environment or could threaten human } \\
\text { life }\end{array}$ & $9 / 30 / 2000$ \\
\hline & & & $\begin{array}{l}\text { Visually inspect } 100 / 200 / 300 / 400 / 1100 \\
\text { areas \& Columbia River bank for } \\
\text { malfunctions, deteriorations, operator } \\
\text { errors, and discharges that could } \\
\text { cause or lead to the release of } \\
\text { dangerous waste constituents to the } \\
\text { environment or could threaten human } \\
\text { life }\end{array}$ & $9 / 30 / 2001$ \\
\hline
\end{tabular}




\subsection{MYWP Update Summary}

This summary provides a basis for listing the changes to the baseline. In the current work scope for the Environmental Compliance Program, no new work scope exists. However additional work scope has been requested of the program. This request for additional work scope will be addressed using the baseline change control process after fiscal year 1999 commences.

\subsection{Baseline Change Request}

A baseline change request to conduct current, planned work scope is not needed for the Environmental Compliance Program in FY99. 


\section{CORRESPONDENCE DISTRIBUTION COVERSHEET}

Autinor

W. D. Adair, FDH

K. A. Giese, FDH, 3̦76-8301
Acioressee

J. E. Rasmussen, RL
Correspondence ivo.

FDH-9855103 A R2

Subject: FISCAL YEAR 1999 FINAL/APPROVED MULTI-YEAR WORK PLAN

\section{DISTRIBUTION}

\begin{tabular}{|c|c|c|c|c|}
\hline Approval & Date & Name & Location & w/ \\
\hline \multirow{29}{*}{ 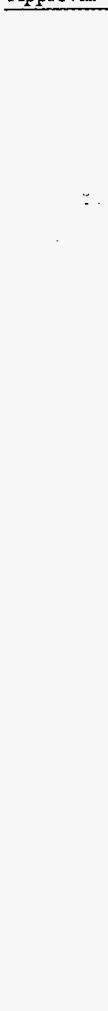 } & & Correspondence Control & A3-01 & $\mathrm{X}$ \\
\hline & & Fluor Daniel Hanford, Inc. & & \\
\hline & & President's Office & $\mathrm{H} 5-20$ & \\
\hline & & W. D. Adair & H6-21 & $X$ \\
\hline & & L. D. Arnold & $\mathrm{H} 8-67$ & $\mathrm{X}$ \\
\hline & & B. A Austin & $\mathrm{H} 6-67$ & \\
\hline & & S. M. Cooley & H8-67 & $X$ \\
\hline & & K. A. Giese & H6-21 & $X$ \\
\hline & & R. T. Hansen & H8-46 & \\
\hline & & O. S. Kramer & H8-67 & $X$ \\
\hline & & D. L. Mitchell & $\mathrm{H} 6-23$ & $X$ \\
\hline & & S. M. Price & H6-23 & $\mathrm{X}$ \\
\hline & & D. G. Ranade & H6-23 & $X$ \\
\hline & & R. L. Shoup & H8-67 & $X$ \\
\hline & & K. B. Iobier (5) & $\mathrm{B} 3-53$ & $X$ \\
\hline & & B. D. Williamson & B3-15 & \\
\hline & & Central Files & B1-07 & $X$ \\
\hline & & Document 2 rocessing Center & A3-94 & $X$ \\
\hline & & Hanford Technical Library & P8-55 & $X$ \\
\hline & & Numatec Hanford Corporatio & & \\
\hline & & K. E. Carpenter & $\mathrm{R} 3-47$ & $\mathrm{X}$ \\
\hline & & U.S. Department of Energy, $\mathrm{B}$ & hland $\mathrm{Op}$ & \\
\hline & & C. E. Clark & A5-15 & $X$ \\
\hline & & J. B. Hall (1 for Bay) & A5-15 & $X$ \\
\hline & & R. N. Krekei & A5-15 & $x$ \\
\hline & & P. W. Kruger & A5-54 & $X$ \\
\hline & & G. H. Sanders & A5-15 & $\mathrm{X}$ \\
\hline & & P.F. X. Dunigan & A5-15 & $X$ \\
\hline & & J. L. Ward & A7-89 & $\mathrm{X}$ \\
\hline
\end{tabular}

Waste Management Federal Services of Hanford Inc.

\begin{tabular}{lll}
\hline G. D. Cummins & H6-26 & X \\
L. P. Diediker & $H 6-36$ & X \\
R. H. Engelmann & $H 6-26$ & X \\
E. M. Greager & $H 6-36$ & X \\
J. O. Skolrud & $H 6-20$ & X \\
J. A. Winterhalder & H6-21 & X
\end{tabular}


Fluor Daniel Hantord, Inc.

P.O. Box 1000

Richland, WA 99352

October 6, 1998

FDH-9855103A R2

Mr. J. E, Rasmussen, Director

Environmental Assurance, Permits, and Policy Division

U.S. Department of Energy A5-15

Richland Operations Office

Post Office Box 550

Richland, Washington 99352

Dear Mr. Rasmussen:

\section{FISCAL YEAR 1999 FINAL/APPROVED MULTI-YEAR WORK PLAAN}

Attached is the final/approved Fiscal Year 1955 ivuli-Year Work Plan (aix wr) iur une Environmentai Compliance Program which reprecents the merging of the Effuent and Environmental Monitoring Prowram and the Hanford Environmental Management Program. The MYYxp ruas prepared in accordañe with the U.S. Dejartinent of Energy, Richland Opcantions Office (RL) guidanace letter, from A. Q. Murphy, RL, to R. F. Green, FDH, "Contract No. DE-AC06-96RL13200-Baseline Updating Guidance (BUG)," 98-PID-641, dated June 11, 1998, and subsequent Fluor Daniel Hanford, Inc. guidance to all Project Hanford Management Contract contractors that was promulgated on the Hanford Intranet. The MYWP was approved by RL on September 15, 1998.

Please contact Mr. K. A. Giese, of my staff, 376-8301, if you have any questions.

Very truly yours,

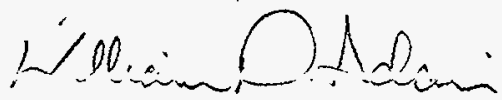

William D. Adair, Director

Environmental Protection

Contracting Officer Representative for

Fluor Daniel Hanford, Inc.

$\operatorname{lgj}$

Attachment 
FDH-9855103 A R2

ATTACHMENT

HNF-SP-1112, Rev. 4, Environmental Compliance Program

FY 1999 Multi-Year Work Plan, WBS 1.8.2.3 